\title{
An HST/COS legacy survey of high-velocity ultraviolet absorption in the Milky Way's circumgalactic medium and the Local Group $\star, \star \star$
}

\author{
P. Richter ${ }^{1,2}$, S. E. Nuza ${ }^{2,3,4}$, A. J. Fox ${ }^{5}$, B. P. Wakker ${ }^{6}$, N. Lehner ${ }^{7}$, N. Ben Bekhti ${ }^{8}$, C. Fechner ${ }^{1}$, M. Wendt ${ }^{1}$, \\ J. C. Howk ${ }^{7}$, S. Muzahid ${ }^{9}$, R. Ganguly ${ }^{10}$, and J. C. Charlton ${ }^{11}$ \\ ${ }^{1}$ Institut für Physik und Astronomie, Universität Potsdam, Karl-Liebknecht-Str. 24/25, 14476 Golm, Germany \\ e-mail: prichter@astro.physik.uni-potsdam.de \\ 2 Leibniz-Institut für Astrophysik Potsdam (AIP), An der Sternwarte 16, 14482 Potsdam, Germany \\ 3 Universidad de Buenos Aires, Facultad de Ciencias Exactas y Naturales, 1053 Buenos Aires, Argentina \\ ${ }^{4}$ CONICET-Universidad de Buenos Aires, Instituto de Astronomía y Física del Espacio (IAFE), Buenos Aires, Argentina \\ Space Telescope Science Institute, 3700 San Martin Drive, Baltimore, MD 21218, USA \\ 6 Supported by NASA/NSF, affiliated with the Department of Astronomy, University of Wisconsin-Madison, \\ 475 North Charter Street, Madison, WI 53706, USA \\ 7 Department of Physics, University of Notre Dame, 225 Nieuwland Science Hall, Notre Dame, IN 46556, USA \\ 8 Fraunhofer Institute for High Frequency Physics and Radar Techniques FHR, Fraunhoferstr. 20, 53343 Wachtberg, Germany \\ 9 Leiden Observatory, University of Leiden, PO Box 9513, 2300 RA Leiden, The Netherlands \\ 10 Department of Computer Science, Engineering, \& Physics, University of Michigan-Flint, Murchie Science Building, \\ 303 Kearsley Street, Flint, MI 48502, USA \\ 11 Department of Astronomy and Astrophysics, Pennsylvania State University, University Park, PA 16802, USA
}

Received 17 November 2016 / Accepted 3 June 2017

\section{ABSTRACT}

\begin{abstract}
Context. The Milky Way is surrounded by large amounts of diffuse gaseous matter that connects the stellar body of our Galaxy with its large-scale Local Group (LG) environment.

Aims. To characterize the absorption properties of this circumgalactic medium (CGM) and its relation to the LG we present the so-far largest survey of metal absorption in Galactic high-velocity clouds (HVCs) using archival ultraviolet (UV) spectra of extragalactic background sources. The UV data are obtained with the Cosmic Origins Spectrograph (COS) onboard the Hubble Space Telescope (HST) and are supplemented by $21 \mathrm{~cm}$ radio observations of neutral hydrogen.

Methods. Along 270 sightlines we measure metal absorption in the lines of Si II, Si III, C II, and C IV and associated H I $21 \mathrm{~cm}$ emission in HVCs in the velocity range $\left|v_{\mathrm{LSR}}\right|=100-500 \mathrm{~km} \mathrm{~s}^{-1}$. With this unprecedented large HVC sample we were able to improve the statistics on HVC covering fractions, ionization conditions, small-scale structure, CGM mass, and inflow rate. For the first time, we determine robustly the angular two point correlation function of the high-velocity absorbers, systematically analyze antipodal sightlines on the celestial sphere, and compare the HVC absorption characteristics with that of damped Lyman $\alpha$ absorbers (DLAs) and constrained cosmological simulations of the LG (CLUES project).

Results. The overall sky-covering fraction of high-velocity absorption is $77 \pm 6$ percent for the most sensitive ion in our survey, Si III, and for column densities $\log N(\mathrm{Si}$ III $) \geq 12.1$. This value is $\sim 4-5$ times higher than the covering fraction of $21 \mathrm{~cm}$ neutral hydrogen emission at $\log N(\mathrm{HI}) \geq 18.7$ along the same lines of sight, demonstrating that the Milky Way's CGM is multi-phase and predominantly ionized. The measured equivalent-width ratios of Si II, Si III, C II, and C IV are inhomogeneously distributed on large and small angular scales, suggesting a complex spatial distribution of multi-phase gas that surrounds the neutral $21 \mathrm{~cm} H V C s$. We estimate that the total mass and accretion rate of the neutral and ionized CGM traced by HVCs is $M_{\mathrm{HVC}} \geq 3.0 \times 10^{9} M_{\odot}$ and $\mathrm{d} M_{\mathrm{HVC}} / \mathrm{d} t \geq 6.1 M_{\odot} \mathrm{yr}^{-1}$, where the Magellanic Stream (MS) contributes with more than 90 percent to this mass/mass-flow. If seen from an external vantage point, the Milky Way disk plus CGM would appear as a DLA that would exhibit for most viewing angles an extraordinary large velocity spread of $\Delta v \approx 400-800 \mathrm{~km} \mathrm{~s}^{-1}$, a result of the complex kinematics of the Milky Way CGM that is dominated by the presence of the MS. We detect a velocity dipole of high-velocity absorption at low/high galactic latitudes that we associate with LG gas that streams to the LG barycenter. This scenario is supported by the gas kinematics predicted from the LG simulations.

Conclusions. Our study confirms previous results, indicating that the Milky Way CGM contains sufficient gaseous material to feed the Milky Way disk over the next Gyr at a rate of a few solar masses per year, if the CGM gas can actually reach the MW disk. We demonstrate that the CGM is composed of discrete gaseous structures that exhibit a large-scale kinematics together with small-scale variations in physical conditions. The MS clearly dominates both the cross section and mass flow of high-velocity gas in the Milky Way's CGM. The possible presence of high-velocity LG gas underlines the important role of the local cosmological environment in the large-scale gas-circulation processes in and around the Milky Way.
\end{abstract}

Key words. Galaxy: halo - Galaxy: structure - Galaxy: evolution - ISM: kinematics and dynamics - techniques: spectroscopic ultraviolet: ISM

* Based on observations obtained with the NASA/ESA Hubble Space Telescope, which is operated by the Space Telescope Science Institute (STScI) for the Association of Universities for Research in Astronomy, Inc., under NASA contract NAS5D26555.

$\star \star$ Full Tables A.1, A.2, and A.4 are available at the CDS via anonymous ftp to cdsarc.u-strasbg.fr $(130.79 .128 .5)$ or via http://cdsarc.u-strasbg.fr/viz-bin/qcat?J/A+A/607/A48 


\section{Introduction}

Observational and theoretical studies indicate that a substantial (if not dominant) fraction of the diffuse gaseous material in spiral galaxies is situated outside the disk in an extended halo that reaches out to the virial radius $\left(R_{\text {vir }}\right)$. This extraplanar diffuse gas component is commonly referred to as the circumgalactic medium (CGM). Beyond the virial radius there also exists a large amount of diffuse gas that is gravitationally bound to the large-scale cosmological environment in which the galaxies reside (Wakker et al. 2015). Depending on the nature of this cosmological environment, the diffuse gas beyond $R_{\mathrm{vir}}$ is called the intergalactic medium (IGM), or the intragroup/intracluster medium in case of an IGM bound in galaxy groups and clusters. The life cycle of such gas is determined by cosmological structure formation, infall and outflow from galaxies, and galaxy merging. As a result, gas far beyond the stellar bodies of galaxies spans large ranges in physical conditions and chemical abundances, but also represents a major baryon reservoir (e.g., Fox et al. 2014; Tumlinson et al. 2013; Werk et al. 2013; Liang \& Chen 2014; Nuza et al. 2014; Richter et al. 2011, 2016).

The Milky Way is known to also be surrounded by diffuse gas that originates in the Galaxy's CGM and in the Local Group (LG) (see Wakker \& van Woerden 1998; Richter 2006, 2017; Putman et al. 2012, for reviews). Recent studies further indicate that M31 also has an extended gaseous halo (Lehner et al. 2015). In contrast to more distant galaxies, the gaseous outskirts of the Milky Way and M31 can be studied in great detail owing to the large amounts of emission and absorption-line data that are available from various instruments, as well as from simulations (e.g., Fox et al. 2014; Nuza et al. 2014, hereafter refered to as N14). Studies that focus on characterizing the nature of the Milky Way's CGM and its connection to LG gas and M31 thus are crucial for our general understanding of circumgalactic gas in the local Universe.

As a result of our position within the disk of the Milky Way, the local CGM/IGM can be identified most efficiently from its kinematics, as the bulk of the gas does not participate in the rotational motion of the Galaxy's thin and thick disk (e.g., Wakker $\&$ van Woerden 1998). The characteristic local standard of rest (LSR) velocity range of gas in the Milky Way's CGM and in the LG thus lies between $\left|v_{\text {LSR }}\right|=50$ and $450 \mathrm{~km} \mathrm{~s}^{-1}$ (Wakker \& van Woerden 1998), although CGM gas at lower velocities might exist (Peek et al. 2009; Zheng et al. 2015). The Dopplershifted CGM therefore can be observed either through emission or absorption lines of hydrogen and heavy elements with spectrographs that exhibit sufficient spectral resolution. In addition to radio observations that can be used to pinpoint the amount of predominantly neutral gas in the local CGM using the $21 \mathrm{~cm}$ hyperfine structure transition of $\mathrm{HI}$, ultraviolet (UV) and optical absorption spectra of extragalactic background sources are extremely useful to study metal-ion absorption in both neutral and ionized gas down to very low gas column densities. UV satellites such as the Far Ultraviolet Spectroscopic Explorer (FUSE) and the various UV spectrographs installed on Hubble Space Telescope (HST) (e.g., the Space Telescope Imaging Spectrograph, STIS, and Cosmic Origins Spectrograph (COS)) have been very successful in providing large amounts of UV absorption-line data to study the CGM of the Milky Way in great detail (e.g., Wakker et al. 2003; Sembach et al. 2003; Lehner et al. 2012; Herenz et al. 2013; Fox et al. 2014).

In the canonical classification scheme of the Milky Way's circumgalactic gas components one defines the so-called "highvelocity clouds" (HVCs) as gaseous structures that are observed in $\mathrm{HI} 21 \mathrm{~cm}$ radio emission or in line-absorption against extragalactic background sources at LSR velocities $\left|v_{\mathrm{LSR}}\right| \geq$ $100 \mathrm{~km} \mathrm{~s}^{-1}$. The intermediate-velocity clouds (IVCs) represent extra-planar gaseous features at lower radial velocities $\left(\left|v_{\mathrm{LSR}}\right|=\right.$ $50-100 \mathrm{~km} \mathrm{~s}^{-1}$ ). IVCs are often related to gas in the disk-halo interface at low vertical distances from the disk $(|z| \leq 2 \mathrm{kpc}$; Wakker 2001).

Over the last decades there has been substantial progress in characterizing the role of the Galactic CGM in the on-going evolution of the Milky Way. The combination of $21 \mathrm{~cm}$ and UV spectral data allowed researchers to pinpoint the chemical composition of the gas and its physical conditions (Wakker et al. 1999, 2003; Sembach et al. 2003; Richter et al. 1999, 2001a,b,c, 2009, 2012, 2013; Tripp et al. 2003; Fox et al. 2005, 2006, 2010, 2013, 2014; Ben Bekhti et al. 2008, 2012; Lehner et al. 2010, 2011, 2012; Collins et al. 2009; Shull et al. 2009). In general, the Milky Way's CGM is metal-enriched with $\alpha$ abundances in the range 0.1-1.0 solar (e.g., Wakker et al. 1999; Richter et al. 2001b; Fox et al. 2013). The presence of low and high ions in the gas reflects its extreme multi-phase nature with temperatures ranging from $T=10^{2}$ to $10^{7} \mathrm{~K}$. Recent studies imply that the bulk of the Milky Way CGM baryons reside in a diffuse (at gas densities $n_{\mathrm{H}} \leq 10^{-3} \mathrm{~cm}^{-3}$ ), predominantly ionized gas phase that can be detected in UV and X-ray absorption lines of intermediate and high ions against extragalactic background point sources (e.g., Sembach et al. 2003; Wakker et al. 2003; Shull et al. 2009; Richter et al. 2008, 2009; Lehner et al. 2012; Miller et al. 2016). Another important finding from absorption spectroscopy is that most of the spatially extended neutral gas features that are seen in $\mathrm{HI} 21 \mathrm{~cm}$ emission are relatively nearby at distances $d<15 \mathrm{kpc}$ (Ryans et al. 1997; Wakker et al. 2007, 2008; Thom et al. 2006, 2008; Lehner et al. 2012; Richter et al. 2015), ruling out that that these objects represent pristine, extragalactic gas clouds (see discussion in Blitz et al. 1999). Many of the ionized HVCs are located at similarly small distances deep within the potential well of the Milky Way (Lehner \& Howk 2011; Lehner et al. 2012), but some of observed high-ion absorbers possibly are related to LG gas (Sembach et al. 2003; Nicastro et al. 2003).

The only neutral high-velocity structure in the Galactic CGM that clearly reaches beyond $30 \mathrm{kpc}$ distance is the MS, a massive stream $\left(10^{8}-10^{9} M_{\odot}\right)$ of neutral and ionized gas that originates from the interaction of the two Magellanic Clouds as they approach the Milky Way (Dieter 1971; Wannier \& Wrixon 1972; Mathewson et al. 1974; D’Onghia \& Fox 2016). Our recent studies of the MS based on UV data from FUSE, HST/STIS and HST/COS (Fox et al. 2010, 2013, 2014; Richter et al. 2013) indicate that the MS contains more gas than the Small and Large Magellanic Clouds, feeding the Milky Way over the next 0.51.0 Gyr with large amounts of mostly metal-poor gas.

In this paper, we present the so-far largest survey of UV absorption features in the Milky Way's CGM and in the LG using archival HST/COS data of 270 extragalactic background sources. The aim of this paper is to pinpoint the absorption characteristics of the Milky Way's CGM and gas in the LG based on proper statistics, to explore the overall physical conditions and the distribution of different gas phases in the gaseous outskirts of our Galaxy, to estimate the total gas mass in HVCs and the Milky Way's gas accretion rate, and to compare the absorption properties of the Milky Way disk+CGM with that of other galaxies. The HVC absorption catalog presented here provides an excellent data base for the comparison between the Milky Way CGM and circumgalactic gas around other low- $z$ galaxies, as traced by intervening absorption-line systems (e.g., Prochaska 2017). 
Since massive gas streams from merger events like the MS are rare in low redshift galaxies, we provide statistical results on the absorption properties of the Milky Way CGM including and excluding the MS contribution. Note that in this paper we do not investigate the chemical composition of the Galactic CGM, dust depletion patterns in the CGM, the role of outflows, or detailed ionization conditions in individual HVCs; these aspects will be presented in forthcoming studies.

This paper is organized as follows: in Sect. 2 we describe the observations, the data reduction and the analysis method. In Sect. 3 we characterize the global absorption properties of the Milky Way CGM and LG gas. The distribution of equivalent widths and column densities of different metal ions is presented in Sect. 4. In Sect. 5 we discuss structural properties of the CGM (HVC complexes, relation to LG galaxies and LG gas, small-scale structure). An estimate of the HVC ionized gas content, total mass, and accretion rate is presented in Sect.6. In Sect. 7 we relate the absorption characteristics of the Milky Way disk+halo to that of damped Lyman $\alpha$ absorbers (DLAs) and compare the observations with predictions from constrained cosmological simulations of the LG. Finally, we conclude our study in Sect. 8.

\section{Observations, data handling, and analysis method}

\subsection{COS spectra selection and data reduction}

In this study we use archival HST/COS data that were retrieved from the HST Science Archive at the Canadian Astronomy Data Centre (CADC). For our analysis we concentrate on absorption lines of low, intermediate, and high ions from silicon and carbon (Si II, Si III, C II, and C IV). Si II, Si III, and C II have strong transitions in the wavelength range between 1190 and $1410 \AA$, as listed in Table 1. This wavelength range is covered by the COS G130M grating that operates from $\lambda=1150-1450 \AA$ at a spectral resolution of $R \approx 15000-20000\left(15-20 \mathrm{~km} \mathrm{~s}^{-1}\right.$ FWHM at a native pixel size of $3 \mathrm{~km} \mathrm{~s}^{-1}$; Green et al. 2012; Debes et al. 2016). For the study of the C IV doublet at 1548.2, $1550.8 \AA$ we require data obtained with the COS G160M grating that covers the range $\lambda=1405-1775 \AA$ (at a spectral resolution similar to that of the G130M grating). In Table 1 we summarize atomic data for the UV transitions of the metal ions that we consider for our analysis (from Morton 2003).

In this paper, we do not analyze UV absorption in other available ions, such as O I and Si IV. Neutral oxygen is an important ion to determine the $\alpha$ abundance in Galactic HVCs (Wakker et al. 1999; Richter et al. 2001a,b; Sembach et al. 2004) and to study the distribution of metal-enriched, neutral gas, as O I and $\mathrm{H}$ I have identical ionization potentials. In our COS data, the only available O I line at $1302.17 \AA$ is contaminated by airglow lines, however. They can be avoided only by using night-only data, which will usually reduce the signal-to-noise ratio $(\mathrm{S} / \mathrm{N})$. In addition, O I $\lambda 1302.17$ is often saturated, so that for most cases where this line is detected only lower limits on the O I column density can be derived. The large range in $\mathrm{S} / \mathrm{N}$ in our data set together with the above listed restrictions limits the diagnostic power of the OI $\lambda 1302.17$ line to a relatively small sub-sample of our sightlines and thus we refrain from considering $\mathrm{O} \mathrm{I}$ in this study. A detailed analysis of O I absorption in HVCs based on HST/COS data instead will be presented in a forthcoming study.

Like C IV, Si IV is a useful tracer of highly ionized gas in and around neutral HVCs, but because of the lower abundance of $\mathrm{Si}$
Table 1. Atomic data for considered UV transitions.

\begin{tabular}{lrr}
\hline \hline Ion & $\lambda_{0}[\AA]$ & $f$ \\
\hline Si III & 1206.50 & 1.6690 \\
Si II & 1190.42 & 0.2502 \\
& 1193.29 & 0.4991 \\
& 1260.42 & 1.0070 \\
& 1304.37 & 0.1473 \\
& 1526.71 & 0.2303 \\
C II & 1334.53 & 0.1278 \\
C IV & 1548.19 & 0.1908 \\
& 1550.77 & 0.0952 \\
\hline
\end{tabular}

Notes. Taken from Morton (2003).

compared to C (Asplund et al. 2009) the detection rate of Si IV at high velocities is relatively small $(<30$ percent in our data; see also Herenz et al. 2013). Therefore, not much additional information is gained from this ion and therefore we also exclude Si IV from our analysis (but show the Si IV absorption in our finding charts; see Fig. 1).

To study high-velocity UV absorption in Si II, Si III, C II, and C IV we searched for all publicly available COS spectra from all types of extragalactic point sources using the CADC web interface. Suitable background sources include various types of AGN and galaxies. G130M/G160M data sets for 552 targets were identified and downloaded by the end of February 2014. The original (raw) data of the individual science exposures were processed by the CALCOS pipeline (v2.17.3) and transformed into standard $\mathrm{x} 1 \mathrm{~d}$ fits files. In a second step, the individual exposures then were coadded using a custom-written code that aligns individual exposures based on a pixel/wavelength calibration. The code considers the relative position of line flanks (for spectra with a $\mathrm{S} / \mathrm{N}$ per resolution element of $>5$ ) or line centers (for spectra with lower $\mathrm{S} / \mathrm{N}$ ) of various interstellar anchor lines that are distributed over the wavelength range of the G130M and G160M gratings. The heliocentric velocity positions of the anchor lines were determined from supplementary H I 21 $\mathrm{cm}$ data from the Effelsberg HI Survey (EBHIS) and GalacticAll Sky Survey (GASS) surveys (see next section). The individual spectra were rebinned and then coadded pixel-by-pixel using the count rate in each pixel; pixels with known artifacts were flagged accordingly and the errors were calculated in the coadded spectra. Finally, we performed a careful visual inspection of the final coadded G130M and G160M spectra to check the quality of the data reduction process.

For the analysis of high-velocity absorption we selected only those spectra that have a minimum $\mathrm{S} / \mathrm{N}$ of 6 per resolution element in the wavelength range between 1208 and $1338 \AA$. This selection criterion reduces the total sample to 270 lines of sight. Quasi stellar object (QSO) names and Galactic coordinates for these 270 sightlines are listed in Table A.1.

\subsection{CGM identification and absorption-line analysis}

For the identification of high-velocity absorption along the 270 COS sightlines we transformed the spectra into a LSR velocity frame. For all sightlines we determined (in an automated fashion) a global continuum level by normalizing the velocity profiles to the highest flux level in the velocity range $\left|v_{\mathrm{LSR}}\right| \leq$ $500 \mathrm{~km} \mathrm{~s}^{-1}$. In this way, we created HVC finding charts that were visually inspected to identify high-velocity absorption in the range $\left|v_{\mathrm{LSR}}\right|=100-500 \mathrm{~km} \mathrm{~s}^{-1}$. In Fig. 1, we show four 


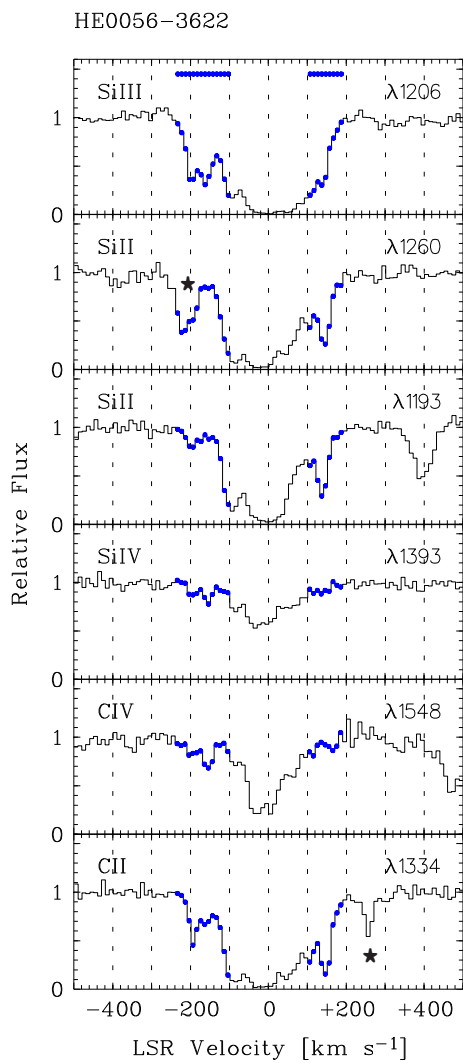

SDSSJ 123304.05-003134.1
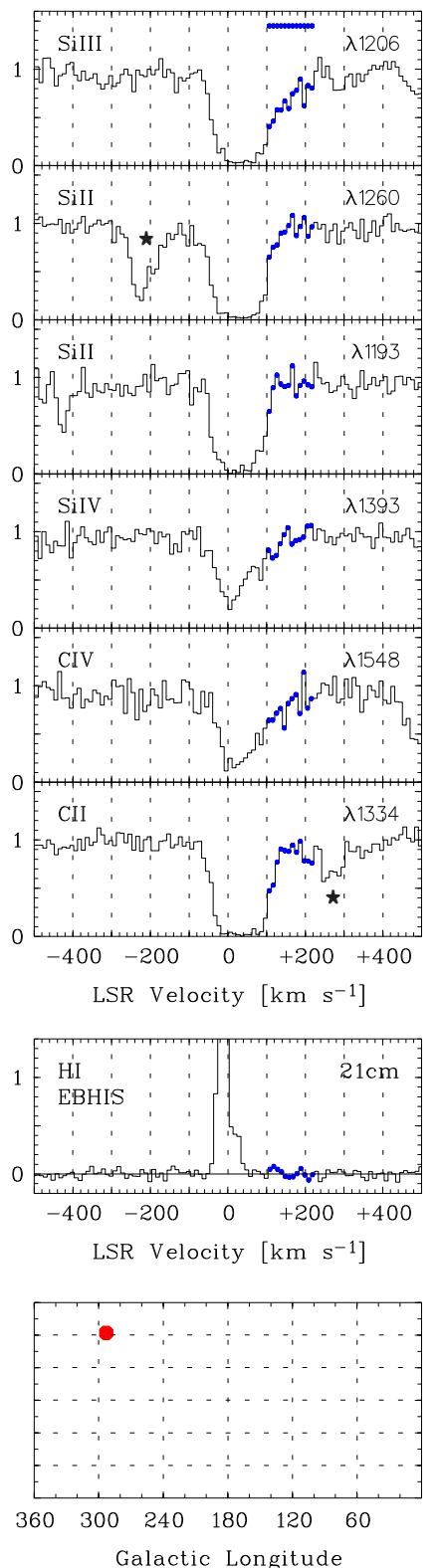

UKS-0242-724
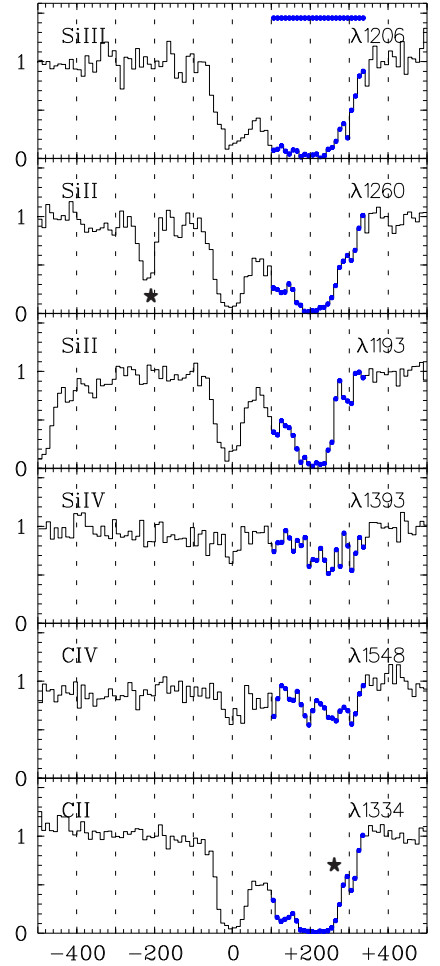

LSR Velocity $\left[\mathrm{km} \mathrm{s}^{-1}\right]$


RXJ $1230.8+0115$
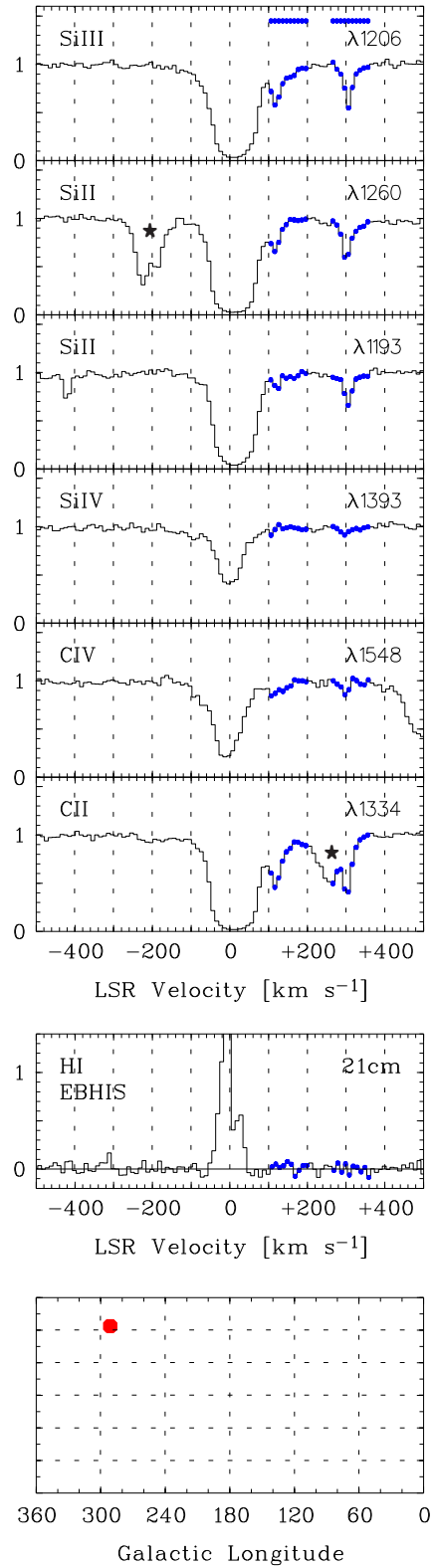

Fig. 1. Examples for HVC finding charts from our survey. In the upper panel the absorption profiles of Si III, Si II, C IV, and C II are shown. For comparison, Si IV absorption is also displayed. Regions with high-velocity absorption are indicated in blue, with the velocity range indicated with the blue bar in the top panel. We further show the $21 \mathrm{~cm}$ emission profile for the same sightlines (middle panel) and the sightline position in the $(l, b)$ plane (lower panel) together with the HVC identification (see Sect. 5.1). The star symbol indicates a blend with another ISM line (S II $\lambda 1259$ in the Si II $\lambda 1260$ panel, C II ${ }^{\star} \lambda 1335$ in the C II $\lambda 1334$ panel). Appendix B contains this plot for all 270 sightlines.

examples of such finding charts. The full set of velocity profiles for all 270 sightlines is shown in Fig. B.2. Note that absorption at lower (absolute) velocities is not considered here (although such gas may belong to the halo), as it would require a careful velocity modeling for each sightline to disentangle disk and halo components, which is clearly beyond the scope of this paper.

We regard a high-velocity absorption feature as a definitive detection if it is convincingly ( $>4 \sigma$ evidence) detected in at least two of the above given metal transitions at $\left|v_{\mathrm{LSR}}\right|=100$ $500 \mathrm{~km} \mathrm{~s}^{-1}$, where we use the formalism to define a local detection limit described in Sect. 3.1. This strategy is similar to our previous surveys (Richter et al. 2009, 2016; Lehner et al. 2012; Herenz et al. 2013), but is more restrictive than other studies where also single-line detections are considered (e.g., Collins et al. 2009; Shull et al. 2009). If a high-velocity feature is seen only in one transition (e.g., as a result of low $\mathrm{S} / \mathrm{N}$, lack of data, or due to blending with IGM lines) we label it as HVC/CGM candidate absorber, but do not further consider it in the statistical analysis unless stated otherwise.

The spectral features then were analyzed using the customwritten line analysis tool span. For each high-velocity absorber the exact shape of the local continuum was determined by a low-order polynomial fit of the global continuum (see above; Figs. 1; B.2) within $\left|v_{\text {LSR }}\right| \leq 1000 \mathrm{~km} \mathrm{~s}^{-1}$. Equivalent widths (and their errors) were determined by a direct pixel integration over the absorption profiles. In a similar fashion, ion column densities (or lower limits) then were derived by integrating over the velocity profiles using the apparent optical depth (AOD) 
method, as originally described in Savage \& Sembach (1991). For possibly saturated absorption features (i.e., features with absorption depths $>0.5$ ) the column density obtained from the AOD method is regarded as lower limit. To minimize the influence of saturation effects we adopted as final column density the value obtained for the weakest available line for each ion that shows well-defined high-velocity absorption features. Note that, because of the extended wings of the COS line-spread function, the equivalent widths and column densities derived in this way could be underestimated by a few percent (see Wakker et al. 2015; Richter et al. 2013).

Since we here consider only absorption in the velocity range $\left|v_{\text {LSR }}\right|=100-500 \mathrm{~km} \mathrm{~s}^{-1}$, we cut away the low-velocity extensions of HVC features near $\left|v_{\mathrm{LSR}}\right|=100 \mathrm{~km} \mathrm{~s}^{-1}$. While this partial velocity integration is unsatisfying, we decided to stick to a strict velocity cut-off at $\left|v_{\mathrm{LSR}}\right|=100 \mathrm{~km} \mathrm{~s}^{-1}$ to avoid introducing a bias in our absorber statistics. Yet, the listed equivalent widths, column densities (and the ratios of these quantities) for absorbers near $\left|v_{\mathrm{LSR}}\right|=100 \mathrm{~km} \mathrm{~s}^{-1}$ need to be interpreted with some caution due to this velocity cut-off.

In view of the limited spectral resolution of the COS instrument we do not further investigate in this survey the velocity-component structure of the detected HVC absorbers. All measured equivalent widths and column densities for the high-velocity absorbers in our sample are summarized in Table A.2. For those high-velocity features that have been studied previously with HST/STIS and HST/COS data (e.g., Richter et al. 2009; Herenz et al. 2013; Fox et al. 2013, 2014) the values derived by us generally are in very good agreement with the previous results.

\subsection{Complementary $\mathrm{H} / 21 \mathrm{~cm}$ data}

We complement our HST/COS absorption-line data with $21 \mathrm{~cm}$ data from different instruments and observing campaigns to investigate the relation between UV absorption and $21 \mathrm{~cm}$ emission along each sightline and to infer the ionization state of the Milky Way CGM.

First, we use $21 \mathrm{~cm}$ data from the GASS (McClure-Griffiths et al. 2009; Kalberla et al. 2010), which was carried out with the $64 \mathrm{~m}$ radio telescope at Parkes. The angular resolution of the GASS data is $\sim 15.6^{\prime}$ with an rms of $57 \mathrm{mK}$ per spectral channel $\left(\Delta v=0.8 \mathrm{~km} \mathrm{~s}^{-1}\right)$. Secondly, we make use of $21 \mathrm{~cm}$ data obtained as part of the new Effelsberg HI Survey (EBHIS), which was carried out on the Effelsberg $100 \mathrm{~m}$ radio telescope (Kerp et al. 2011; Winkel et al. 2010). Compared to GASS, EBHIS data has a slightly higher noise level $(\sim 90 \mathrm{mK})$ and a somewhat lower velocity resolution (channel separation: $\Delta v=1.3 \mathrm{~km} \mathrm{~s}^{-1}$ ), but the angular resolution is higher $\left(\sim 10.5^{\prime}\right)$. The typical $\mathrm{H}$ I column density limit in the $21 \mathrm{~cm}$ data used here is a few times $10^{18}$ $\mathrm{cm}^{-2}$. In general, our $21 \mathrm{~cm}$ data is complete for H I column densities $>5 \times 10^{18} \mathrm{~cm}^{-2}(4 \sigma$ level $)$. Note that there are other, more sensitive $21 \mathrm{~cm}$ surveys for individual regions (e.g., Lockman et al. 2002). For each sightline we included the $21 \mathrm{~cm}$ velocity profile in the HVC finding charts (Figs. 1; B.2).

$\mathrm{H}$ I column densities (and their limits) were determined by integrating the $21 \mathrm{~cm}$ emission profile over the appropriate velocity range (defined by the UV absorption) using the relation

$N(\mathrm{HI})=1.823 \times 10^{18} \mathrm{~cm}^{-2} \int_{v_{\min }}^{v_{\max }} T_{\mathrm{B}} \mathrm{d} v$,

where $T_{\mathrm{B}}$ denotes the brightness temperature (in $[\mathrm{K}]$ ) and the gas is assumed to be optically thin in $21 \mathrm{~cm}$ (Dickey \& Lockman 1990).

\section{Characterization of HVC absorption}

\subsection{Sky distribution}

In Fig. 2 we show the sky distribution of the 270 sightlines in Galactic coordinates $(l, b)$ using a Hammer-Aitoff sky projection centered on $l=180^{\circ}$. Filled circles indicate directions along which high-velocity absorption $\left(\left|v_{\mathrm{LSR}}\right| \geq 100 \mathrm{~km} \mathrm{~s}^{-1}\right)$ is convincingly detected in at least two different UV lines, while crossed filled circles label the tenative detections and open circles the non-detections. The mean radial velocity of the absorption is indicated with the color scheme shown at the bottom of the plot. There is a clear asymmetry in the sky distribution of the LOS: three quarters of the sightlines are located in the northern sky. About 60 percent of the detected HVC absorption features have negative velocities. They are found at $l<210^{\circ}$ and $l>300^{\circ}$ with only a few exceptions. Positive-velocity HVC absorption concentrates in a strip in galactic longitude in the range $l=210$ $300^{\circ}$. There is a striking lack of HVC absorption near the northern galactic pole at $b>75^{\circ}$. The low detection rate of low and intermediate ions in this region has been noted previously (Lehner et al. 2012), but this trend now becomes more significant due to the improved statistics. In the southern part of the sky, gas from the MS dominates the absorption characteristics of high-velocity gas (Fox et al. 2013, 2014). However, some of the negative velocity gas at $l<130^{\circ}$ possibly belongs to the CGM of M31 (Lehner et al. 2015) and to intragroup gas in the Local Group filament, as will be discussed later.

Note that the observed velocity dipole of the high-velocity absorption at high galactic latitudes cannot be explained alone by galactic rotation, because along many sightlines $\cos (b) v_{\text {rot }}$ is smaller than the observed absorption velocities, even if a high circular orbit speed of the Sun of $v_{\text {rot }}=255 \mathrm{~km} \mathrm{~s}^{-1}$ is assumed (Reid et al. 2014). For MS absorption in the south, in particular, the velocity dipole instead suggests a non-circular orbit of the Stream around the Milky Way (Putman et al. 2003).

We securely detect 187 HVC absorbers along the 270 sightlines, translating to a detection rate of high-velocity absorption of $f_{\text {det }}=69 \pm 5$ percent. If we add the $37 \mathrm{HVC}$ candidates (tentative detections, see above), the detection rate increases to $83 \pm 6$ percent. There are, however, a large number of low-S/N spectra in our sample that are not particularly sensitive to detect weak HVC features.

To take into account the different $\mathrm{S} / \mathrm{Ns}$ in our spectra and to compare our results with previous measurements we need to consider the detection threshold of our COS data in more detail. For each of the four ions considered in this study (C II, C IV, Si II, and Si III) we transformed the individual detection rates, $f_{\text {det }}$, into covering fractions, $f_{\mathrm{c}}\left(N_{\text {lim }}\right)$, where $N_{\text {lim }}$ represents a specific (lower) column-density threshold. As covering fraction we define the number of sightlines that exhibit securely detected high-velocity absorption with a column density $N \geq N_{\text {lim }}$, devided by the total number of sightlines that are sensitive to detect HVC absorption at $N \geq N_{\text {lim }}$. For a spectrum with a given $\mathrm{S} / \mathrm{N}$ per resolution element, a spectral resolution $R$, the $4 \sigma$ limiting column density threshold, $N_{\text {lim }}$, for an unresolved line with oscillator strength $f$ at laboratory wavelength $\lambda_{0}$ (Table 1 ) is given by the expression

$N_{\lim } \geq 1.13 \times 10^{20} \mathrm{~cm}^{-2} \frac{4}{R(\mathrm{~S} / \mathrm{N}) f\left(\lambda_{0} / \AA\right)}$.

Because our COS spectra span a large range in $\mathrm{S} / \mathrm{N}$, they are unequally sensitive to show high-velocity absorption features and thus $f_{\mathrm{c}}\left(N_{\mathrm{lim}}\right)$ is expected to decrease with decreasing $\mathrm{S} / \mathrm{N}$ (and increasing $\left.N_{\text {lim }}\right)$. 


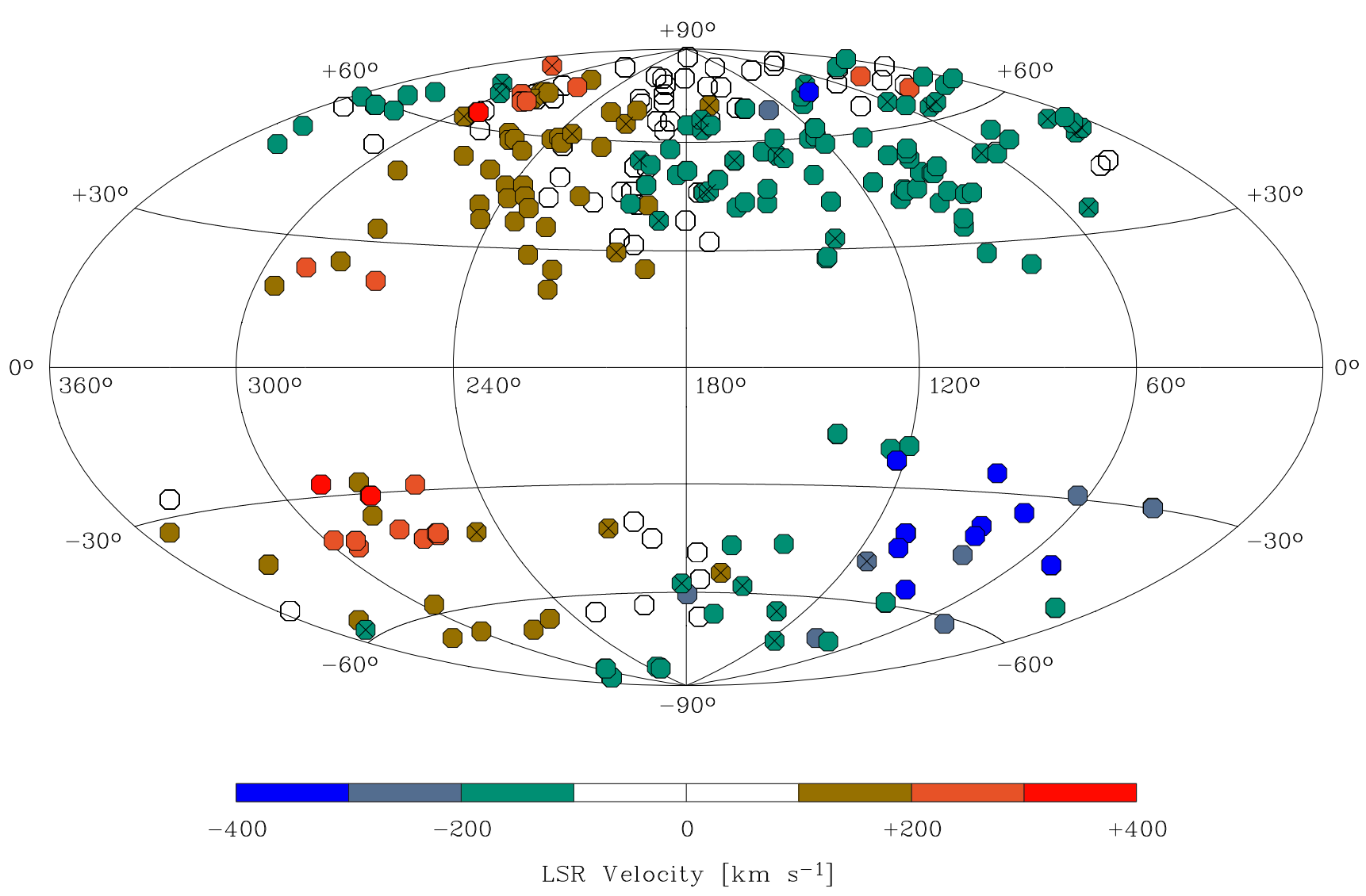

Fig. 2. Sky distribution of sightlines in our COS sample in a Hammer-Aitoff projection of Galactic coordinates. Secure detections of high-velocity gas at $\left|v_{\text {LSR }}\right|=100-500 \mathrm{~km} \mathrm{~s}^{-1}$ are indicated with filled circles, while tentative detections are labeled with crossed filled circles; open circles mark the non-detections. The mean absorption velocities of the dominant absorption components along each sightline are color-coded according to the color scheme at the bottom of the figure.

Table 2. Covering fractions for different ions (in percent).

\begin{tabular}{lcccccccccc}
\hline \hline & & & $f_{\mathrm{c}}$ & $f_{\mathrm{c}}$ & $f_{\mathrm{c}}$ & $f_{\mathrm{c}}$ & $f_{\mathrm{c}}$ & $f_{\mathrm{c}}$ & $f_{\mathrm{c}}$ \\
\hline Ion & $\log N_{\lim }$ & $C^{a}$ & all sky & $b>0^{\circ}$ & $0^{\circ}<b<75^{\circ}$ & $b \geq 75^{\circ}$ & $b \leq 0^{\circ}$ & $-75^{\circ}<b \leq 0^{\circ}$ & $b \leq-75^{\circ}$ & $\begin{array}{c}\text { All sky }_{\text {s }} \\
\text { without MS }\end{array}$ \\
\hline Si III & 12.1 & 0.95 & $77 \pm 6$ & $73 \pm 7$ & $79 \pm 7$ & $19 \pm 11$ & $89 \pm 13$ & $88 \pm 13$ & $55-100$ & $72 \pm 7$ \\
Si II & 12.3 & 0.95 & $70 \pm 6$ & $66 \pm 6$ & $72 \pm 7$ & $19 \pm 11$ & $79 \pm 12$ & $76 \pm 12$ & $55-100$ & $65 \pm 6$ \\
C II & 13.2 & 0.96 & $70 \pm 6$ & $66 \pm 6$ & $72 \pm 7$ & $13 \pm 9$ & $82 \pm 12$ & $80 \pm 13$ & $55-100$ & $66 \pm 6$ \\
C IV & 12.9 & 0.94 & $58 \pm 7$ & $44 \pm 7$ & $50 \pm 9$ & 0 & $73 \pm 12$ & $70 \pm 13$ & $55-100$ & $44 \pm 7$ \\
\hline
\end{tabular}

Notes. Note that only those HVC absorbers are considered that are detected in at least two transitions. ${ }^{(a)}$ Completeness level at $N_{\text {lim }}$; see Sect. 3.1 . ${ }^{(b)}$ See Table 3 for adopted $(l, b)$ ranges.

Having defined $N_{\text {lim }}$, we can study the completeness, $C$, of our QSO sample. For this we relate for each ion the number of sightlines with a given column density threshold, $\mathcal{N}_{N>N_{\text {lim }}}$, to the total number of sightlines, $\mathcal{N}_{\text {tot }}$, along which high-velocity absorption in that ion can be detected:

$C\left(N_{\text {lim }}\right)=\frac{\mathcal{N}_{N>N_{\text {lim }}}}{\mathcal{N}_{\text {tot }}} \leq 1$.

The most sensitive tracer for absorption in our survey is Si III $\lambda 1206.50$, which has a very large oscillator strength (see Table 1; Morton 2003). Si III resides in diffuse ionized gas and traces the CGM around galaxies for a broad range of physical conditions (see Collins et al. 2009; Shull et al. 2009; Richter et al. 2016). In our sample, the Si III all-sky covering fraction in
HVCs is $f_{\mathrm{c}}=77 \pm 6$ percent for $N_{\text {lim }}=12.1$, where the completeness is $C=0.95$ at that column-density level. This value is very similar to the covering fraction of highly-ionized gas traced by O VI (58-85 percent; Sembach et al. 2003). The all-sky covering fractions for the other ions in our survey are lower than for Si III; they are listed in the fourth row of Table 2 together with $\log N_{\text {lim }}$ (second row) and $C$ (third row). Interestingly, the detection rate for C IV is lower than for the singly- and doubly-ionized species. Note that for $\mathrm{C}$ II blending effects with $\mathrm{C} \mathrm{II}^{\star} \lambda 1335.71$ slightly reduce the sensitivity to detect high-velocity $\mathrm{C}$ II in the range $v_{\mathrm{LSR}}=200-300 \mathrm{~km} \mathrm{~s}^{-1}$ (see Fig. 1).

The values for $f_{\mathrm{c}}\left(N_{\text {lim }}\right)$ derived in our survey are very similar to those presented in earlier studies using smaller data samples (Lehner et al. 2012; Herenz et al. 2013). The expected decline of $f_{\mathrm{c}}$ with increasing $N_{\mathrm{lim}}$ is shown for all four ions in Fig. 3 

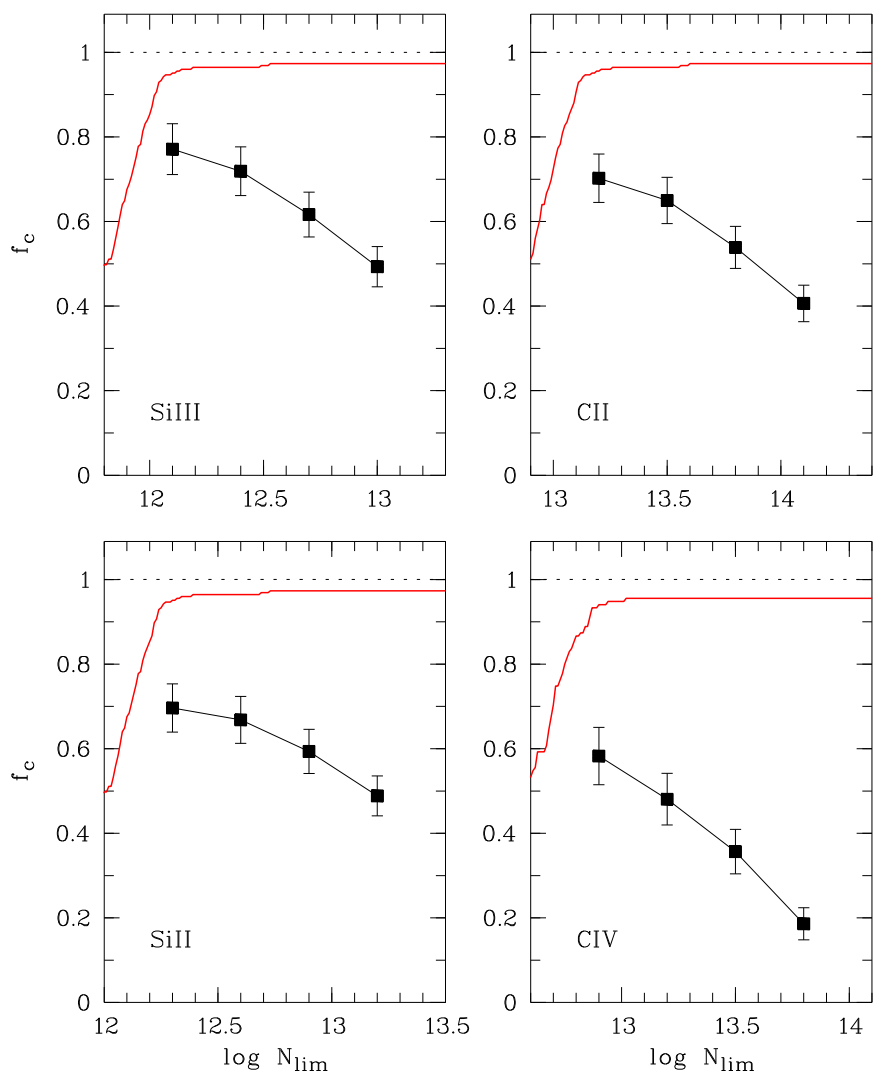

Fig. 3. Covering fractions of high-velocity gas for the four considered ions as a function of limiting column density (filled squares; see also Eq. (2)). The red solid lines indicate the completeness functions for each ion, as defined in Eq. (3).

(filled boxes) together with the completeness function $C\left(N_{\lim }\right)$ (red solid line). For Si III, for instance, $f_{\mathrm{c}}$ declines from 77 to 49 percent if $\log N_{\text {lim }}$ is increased from 12.1 to 13.0. Figure 3 underlines the importance of high $\mathrm{S} / \mathrm{N}$ data for our understanding of the spatial distribution of low-column density gas in the Milky Way's CGM.

In Table 2 we provide additional information on how $f_{\mathrm{c}}$ varies for different latitude bins. In general, the covering fraction of high-velocity gas is slightly smaller in north than in the south $(0.73 \pm 0.07$ vs. $0.89 \pm 0.13$ for Si III), reflecting the fact that the MS covers a significant portion of the southern sky (Fox et al. 2014; Lehner et al. 2012). The absorption fraction reaches 100 percent near the southern galactic pole at $b \leq-75^{\circ}$ (Table 2, last column). Near the northern galactic pole for $b>75^{\circ}$ (thus far away from the MS) the covering fraction is instead only $<20$ percent, marking the most striking difference between the northern and southern high-velocity sky. If we exclude the region covered by the MS (see Table 3 for adopted $(l, b)$ ranges), then the covering fractions are slightly (but not substantially) smaller than the all-sky values (Table 2, last column).

Our study demonstrates that more than three quarters of the sky is covered by diffuse high-velocity gas. Additional absorption-line data for $b<0^{\circ}$ would be desirable to fill the various gaps in the LOS distribution in the southern sky (Fig. 2). Yet, the observed large-scale trends for $f_{\mathrm{c}}$ are statistically robust, indicating an inhomogeneous distribution and a north/south disparity of high-velocity gas on the Galactic sphere.

\subsection{Velocity distribution}

While Fig. 2 gives a general overview of the distribution of highvelocity absorption on the sky at positive and negative radial velocities, it is useful to explore in more detail the distribution of the absorption within different velocity bins. In Fig. 4 we therefore show velocity channel maps of high-velocity absorption in bins of $\Delta v=100 \mathrm{~km} \mathrm{~s}^{-1}$.

The main difference compared to Fig. 2 is that here the entire velocity range of the detected high-velocity absorption is taken into account, while in Fig. 2 only the mean absorption velocity is considered. High-velocity absorption at very high velocities $\left|v_{\text {LSR }}\right|>400 \mathrm{~km} \mathrm{~s}^{-1}$ is seen only at negative velocities, predominantly at $l<140^{\circ}$ and $b<-10^{\circ}$. High-velocity absorption at negative velocities in the northern sky, in contrast, is limited to $v_{\mathrm{LSR}}>-300 \mathrm{~km} \mathrm{~s}^{-1}$. In Sect. 5 we further discuss the origin of these velocity signatures with respect to the different $\mathrm{HVC}$ complexes and gas in the LG.

\subsection{Absorption fraction vs. radial velocity}

An important task is to investigate a possible radial change in the physical conditions of the Milky Way CGM. Ideally, one would study systematically diagnostic metal-ion ratios (such as $\mathrm{Si}$ II/Si III and C II/C IV) as a function of distance to the absorbers. Measuring HVC distances is very difficult, however, and reliable distance estimates have been determined only for a very limited number of halo clouds (Ryans et al. 1997a,b; van Woerden et al. 1998; Wakker et al. 2007, 2008; Thom et al. 2006, 2008; Lehner \& Howk 2011; Lehner et al. 2012; Richter et al. 2015). An alternative approach in this context is to investigate the detection rates (absorption fractions) of the various ions as a function of radial velocity, which provides at least some indirect information on the gas properties of nearby and more distant gas and its radial direction of motion.

In Fig. 5 we have plotted $f_{\text {abs }}$ vs. $v_{\text {LSR }}$ for our HVC absorber sample. It is evident that there are substantial differences between the trends at positive and negative radial velocities. First, the absorption fractions are higher for gas at negative velocities than for positive velocities (at similar sensitivity), a trend that is valid for all of the four considered transitions. This implies that there is more absorbing CGM material that moves towards the Sun than gas that is moving away from it. Secondly, at negative velocities, the absorption fraction of Si III $\lambda 1206.50$ (red line) is always significantly higher than that of $\mathrm{Si}$ II $\lambda 1260.42$ (blue line), while at positive velocities the absorption fractions in both lines are very similar. Thirdly, there is a mild enhancement of $f_{\text {abs }}(\mathrm{C} \mathrm{IV})$ in the range -300 to $-200 \mathrm{~km} \mathrm{~s}^{-1}$ compared to positive velocities.

In Sect. A. 1 we discuss the relation between absorption fraction and LSR velocity excluding the contribution from the MS.

\subsection{Interpretation of observed trends}

The covering fractions for the individual ions, as discussed in the previous subsections, reflect a complex (projected) spatial distribution of the different gas phases in the Galactic halo that are traced by the various low, intermediate, and high ions in our survey. These phases range from cold/neutral gas at relatively high densities $\left(n_{\mathrm{H}} \geq 10^{-3} \mathrm{~cm}^{-3}\right)$ to warm/hot ionized gas at low densities $\left(n_{\mathrm{H}}<10^{-3} \mathrm{~cm}^{-3}\right)$ where the gas cannot recombine efficiently (see review by Richter 2017).

The sky covering fractions indicate that diffuse, predominantly ionized gas, as traced by Si III, as well as Si II, and C II, 

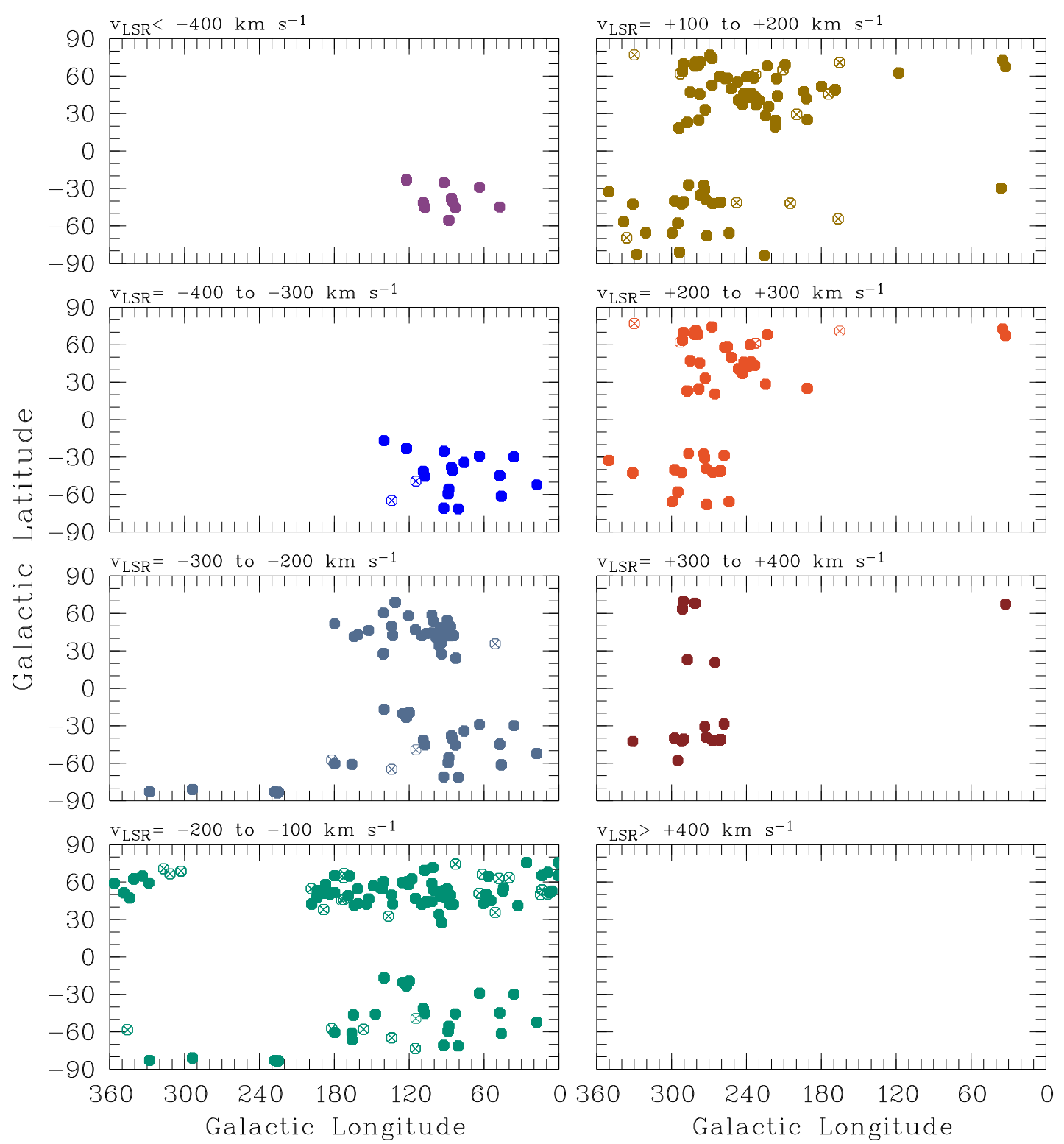

Fig. 4. Velocity channel maps of high-velocity CGM absorbers, indicating the full velocity range in which absorption is observed along each sightline. The velocity range for each panel is labeled above each box. Filled circles indicate confirmed high-velocity absorbers, crossed circles label tentative detections (see Sect. 2.2).

represents the most widespread gas phase in the Milky Way's population of HVCs (see also Shull et al. 2009), which move as coherent circumgalactic structures through the ambient hot coronal gas. The high detection rate of Si III in circumgalactic absorption-line systems at low redshift (Richter et al. 2016) suggests that streams of predominantly ionized gas represent typical features of low-redshift galaxy halos. To reach the observed ion column densities at low gas densities, the absorption path lengths, $d$, in the diffuse ionized gas layers must be large (a few up to a few dozen kpc, typically, as $N_{\text {ion }}=d n_{\text {ion }}$ ). This, together with the large cross section, implies that the diffuse ionized gas phase occupies most of the volume in circumgalactic gas streams, whereas the bulk of the neutral gas (traced by $\mathrm{H} \mathrm{I}$ $21 \mathrm{~cm}$ emission) is confined to specific regions that exhibit the largest gas densities (e.g., Lehner et al. 2012; Joung et al. 2012; Richter 2012). The somewhat lower detection rate of C IV compared to Si III (Table 2) and O VI (Sembach et al. 2003; Wakker et al. 2003) indicates that C IV traces a gas phase in the Milky
Way CGM that is not as widespread as the phase traced by the other two ions, even if one takes into account the only moderate oscillator strengths of the C IV doublet lines (Table 1).

The apparent lack of HVC absorption near the northern galactic pole in our survey and in the study by Lehner et al. (2012) suggests that this region is devoid of neutral and diffuse ionized gas. In contrast, Fox et al. (2006) report the detection of O VI absorption at high positive velocities along several sightlines near the northern galactic pole (their Fig. 1). Together, both results imply that the halo gas near the northern galactic pole is predominantly highly ionized and thus in a phase, that is not traced by the low and intermediate ions considered in this survey.

In general, the physical conditions in the CGM around galaxies are known to be diverse, with temperatures and densities spanning a large range (e.g., Joung et al. 2012; Nuza et al. 2014). On the one hand, they are governed by kinematically complex (and highly dynamic) gas circulation processes (infall, outflow, tidal interactions) that create an inhomogeneous, irregular 


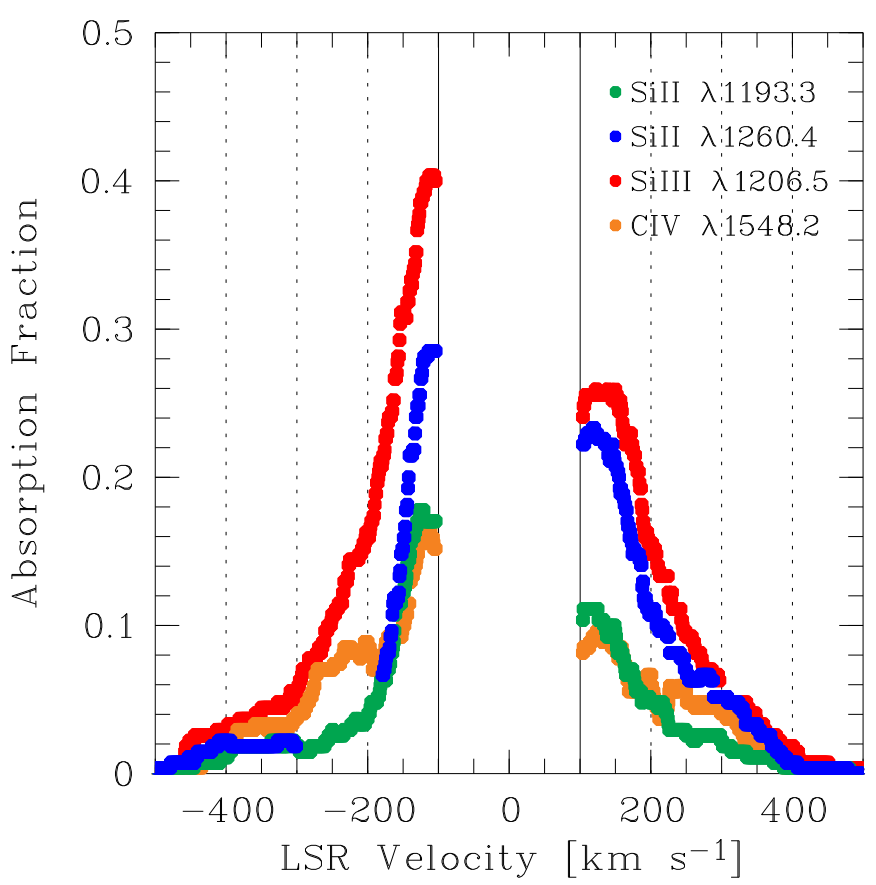

Fig. 5. Absorption fraction (detection rate) of the transitions of Si III $\lambda 1206.5$ (red), Si II $\lambda 1260.4$ (blue), Si II $\lambda 1193.3$ (green), and C IV $\lambda 1548.2$ (orange) as a function of LSR velocity. C II $\lambda 1334.5$ is not included because of severe blending with $\mathrm{C}$ II $^{\star} \lambda 1335.7$ at positive velocities. The gap near $-250 \mathrm{~km} \mathrm{~s}^{-1}$ for Si II $\lambda 1260.4$ is due to blending with Galactic S II $\lambda 1259.5$.

distribution of gaseous matter around Milky-Way type galaxies (see review by Richter 2017). On the other hand, the physical conditions in the CGM are also expected to change gradually from the inside-out owing to declining depth of the gravitational potential at larger distances and the resulting decreasing (equilibrium) gas pressure (see, e.g., Miller \& Bregman 2015). For isothermal gas one would expect to see a declining (mean) gas density at larger distances, which - depending on the radial decline of the ionizing radiation field - possibly results in a higher degree of ionization in the outer halo. Indeed, such a gradual increase in the degree of ionization with increasing galactocentric distance is possibly visible in the CGM of M31 (Lehner et al. 2015) and other low-redshift galaxies (e.g., Werk et al. 2013).

For the Milky Way halo, Lehner \& Howk (2011) demonstrated that the covering fraction of low and high ions in HVCs with $\left|v_{\mathrm{LSR}}\right|<180 \mathrm{~km} \mathrm{~s}^{-1}$ towards halo stars with $d<20 \mathrm{kpc}$ are similar to those derived against QSOs, implying that a large fraction of the CGM gas at low velocities resides relatively nearby in the lower Milky Way halo, while most of gas at very high velocities resides in the outer halo. In view of this trend, Fig. 5 suggests that the observed absorption fraction of ionized gas at high velocities in our survey is due to gas located at large distances from the Galactic plane. Moreover, the observed excess of Si III and C IV compared to Si II at $v_{\text {LSR }}<-200 \mathrm{~km} \mathrm{~s}^{-1}$ suggests that there is more diffuse ionized gas in the CGM at negative velocities compared to positive velocities. As we show in the Appendix (Sect. A.1), much of this negative-velocity gas is related to the MS, which has a very large cross section on the sky (see Sect.5.1; Fox et al. 2014). In addition, some of this ionized material at high negative velocities possibly is related to UV-absorbing LG gas in the general direction of the LG barycenter (see Sembach et al. 2003). This scenario will be further discussed in Sect. 5.4.

\section{Distribution of equivalent widths and column densities}

\subsection{Equivalent widths and column densities of low and high ions}

In the upper panels of Fig. 6 we show the equivalent width distribution of Si III $\lambda 1206.5$, Si II $\lambda 1260.4$, C II $\lambda 1334.5$, and CIV $\lambda 1548.2$ for the 187 securely detected high-velocity absorption components in our survey. Note that these equivalent widths are derived from integrating over the entire velocity range without taking any component structure into account. The equivalent-width distributions for these four ions are very similar to each other, with a peak at low equivalent widths between 0 and $150 \mathrm{~m} \AA$ and a rapid decline towards larger equivalent widths. The majority ( 58 percent) of the absorbers have equivalent widths $<200 \mathrm{~m} \AA$ in the Si III $\lambda 1206.5$ line (69 percent for Si II $\lambda 1260.4,56$ percent for $\mathrm{C}$ II $\lambda 1334.5$, and 78 percent for $\mathrm{C}$ IV $\lambda 1548.2$ ). The equivalent-width distribution of Si III $\lambda 1206.5$ in Galactic high-velocity absorbers mimics that of intervening Si III absorbers at $z=0-0.1$, which are believed to trace the CGM of low-redshift galaxies (Richter et al. 2016; their Fig. 2).

In the lower panels of Fig. 6 we show histograms of the derived ion column densities (green) and their lower limits (gray) based on the AOD analysis (see Sect. 2). Only for Si II and C IV is there more than one transition available (and C IV absorption is generally weak in HVC absorbers), so that only for these two ions can saturation effects be minimized by using for each absorber the weakest detected line for the determination of $\log N$. As a result, the gray-shaded area for these ions in Fig. 6 is smaller than for Si III and C II.

\subsection{Equivalent-width ratios and column-density ratios}

As discussed above, the absorption fractions for the different ions in high-velocity absorbers at different radial velocities (Fig. 5) indirectly indicate a non-uniform radial distribution of different gas phases in Milky Way's circumgalactic environment. Another strategy to explore the large-scale ionization structure of the absorbing gas in our sample is to investigate equivalentwidth ratios of low/intermediate/high ions in different regions of the sky and/or in different velocity bins along sightlines, where these different ions are detected simultaneously.

In Fig. 7 we show the spatial distribution of the equivalentwidth ratios (Si II $\lambda 1260.4 / \mathrm{Si}$ III $\lambda 1206.5$ ), (Si II $\lambda 1193.3 /$

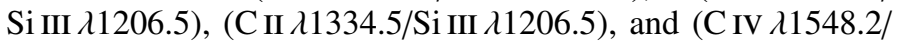
Si III $\lambda 1206.5)$. We use Si III as reference ion as it arises in gas spanning a wide range in physical conditions. Si III thus represents a robust tracer for the ionized gas column of metal-enriched circumgalactic gas at $T<10^{5} \mathrm{~K}$ (see Richter et al. 2016). For comparison, we also show the respective column-density ratios for these ions in Fig. 7 assuming optically thin absorption (i.e., saturation effects are ignored).

The sky distribution of the equivalent-width ratios shows some interesting trends. In the northern hemisphere, there are many sightlines that exhibit high $\mathrm{Si}$ II/Si III and C II/Si III ratios $>1$. As will be discussed in Sect.5.1 (Fig. 8) many of these sightlines coincide spatially with prominent northern 21 cm HVCs, such as Complex C and Complex A. High Si II/Si III and $\mathrm{C}$ II/Si III ratios are observed, however, also in the many positive-velocity absorbers at $l=220^{\circ}-300^{\circ}, b>35^{\circ}$ that have little or no associated $21 \mathrm{~cm}$ emission (see Fig. 8). Also high-velocity absorbers with substantially smaller Si II/Si III and 

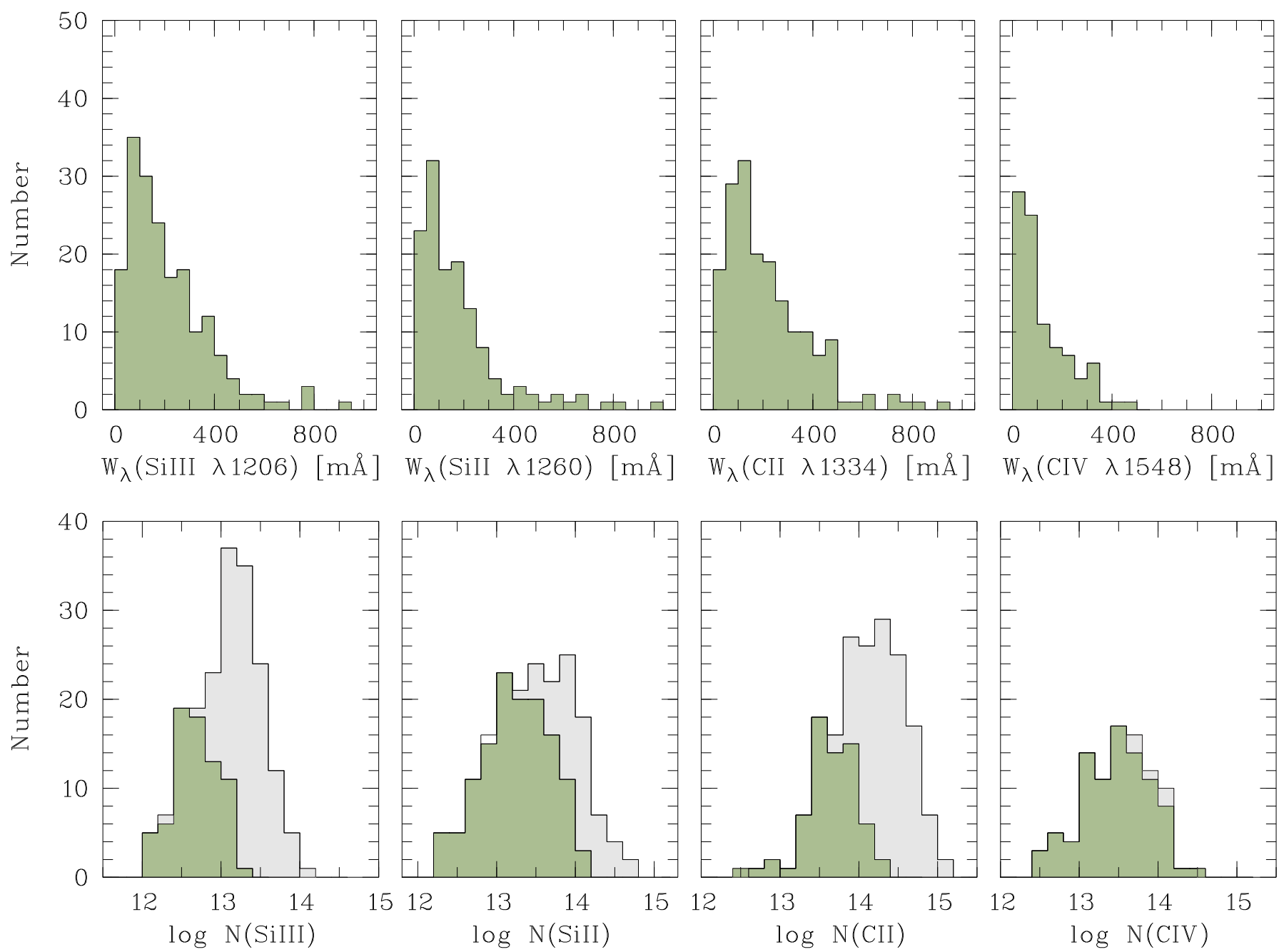

Fig. 6. Upper panel: equivalent-width distributions for the high-velocity absorbers in our COS sample for four different ions. Lower panel: histograms of logarithmic ion column densities, as derived via the AOD method (Savage \& Sembach 1991). The green-shaded area denotes absolute values, the gray-shaded area indicates lower limits for $\log N$ (saturated absorbers).

$\mathrm{C}$ II/Si III ratios are present at $b>0^{\circ}$. C IV absorption is predominantly weak in the northern hemisphere when compared to Si III; only along a handful of sightlines (in directions away from the $21 \mathrm{~cm} \mathrm{HVCs)}$ are the $\mathrm{CIV} / \mathrm{Si}$ III equivalent ratios high.

The situation is quite different in the southern hemisphere. Despite the fact that there are significantly fewer sightlines to be analyzed at $b<0^{\circ}$, it is possible to discern two distinct regions in Fig. 7 with opposite trends. For the sightlines at $l>240^{\circ}, b<$ $0^{\circ}$, the observed Si II $\lambda 1260.4 / \mathrm{Si}$ III $\lambda 1206.5$ equivalent ratios are predominantly $>1$, tracing gas that is associated with the $21 \mathrm{~cm}$ emission from the MS (see Sect. 5). For $l<180^{\circ}, b<0^{\circ}$, in contrast, the same ratio is predominantly small, while the C IV/Si III ratio is coherently larger than in any other region in the northern or southern sky. In Sect. 4.4 we further discuss the physical origin of these large variations in the observed equivant-width ratios.

\subsection{Relation between UV absorption and $21 \mathrm{~cm}$ emission}

In Fig. 8 we again show the sky distribution of high-velocity absorbers and their mean LSR velocities (as in Fig. 2), but now overlaid on the H $21 \mathrm{~cm}$ emission map from the LAB survey using the same color coding for the LSR velocities (except for the range $\left|v_{\mathrm{LSR}}\right| \leq 100 \mathrm{~km} \mathrm{~s}^{-1}$, which is indicated in green for the $21 \mathrm{~cm}$ data and ignored in our absorption-line analysis). As expected, emission and absorption features coincide in velocity, demonstrating that they trace the same overall gas distribution. In all regions of the sky, UV absorption is observed beyond the outer boundaries of $21 \mathrm{~cm} \mathrm{HVCs}$, indicating that the neutral HVCs are surrounded by extended gaseous envelopes (traced only in absorption) that have low neutral gas column densities.

There are regions that are almost devoid of high-velocity H I, but show strong absorption in the UV. The most prominent of such regions is, again, the region $l<180^{\circ}, b<0^{\circ}$, which appears to contain mostly ionized gas at extreme negative velocities (see above). Another prominent region in this context is at $l=200^{\circ}-260^{\circ}, b>0^{\circ}$, which shows UV absorption at high positive velocities, but almost no high-velocity $21 \mathrm{~cm}$ emission. Most of the absorbers in this area have (mean) radial velocities $<200 \mathrm{~km} \mathrm{~s}^{-1}$ and belong to HVC complex WA (see Sect. 5.1). However, there is also a group of absorbers in this region with $v_{\text {LSR }}>200 \mathrm{~km} \mathrm{~s}^{-1}$ that possibly trace LG gas (Sect. 7).

There are also a few absorbers above the Galactic center at $l<50^{\circ}, b>30^{\circ}$ that have no H I $21 \mathrm{~cm}$ counterpart. These absorbers might be related to a large-scale outflow from the 

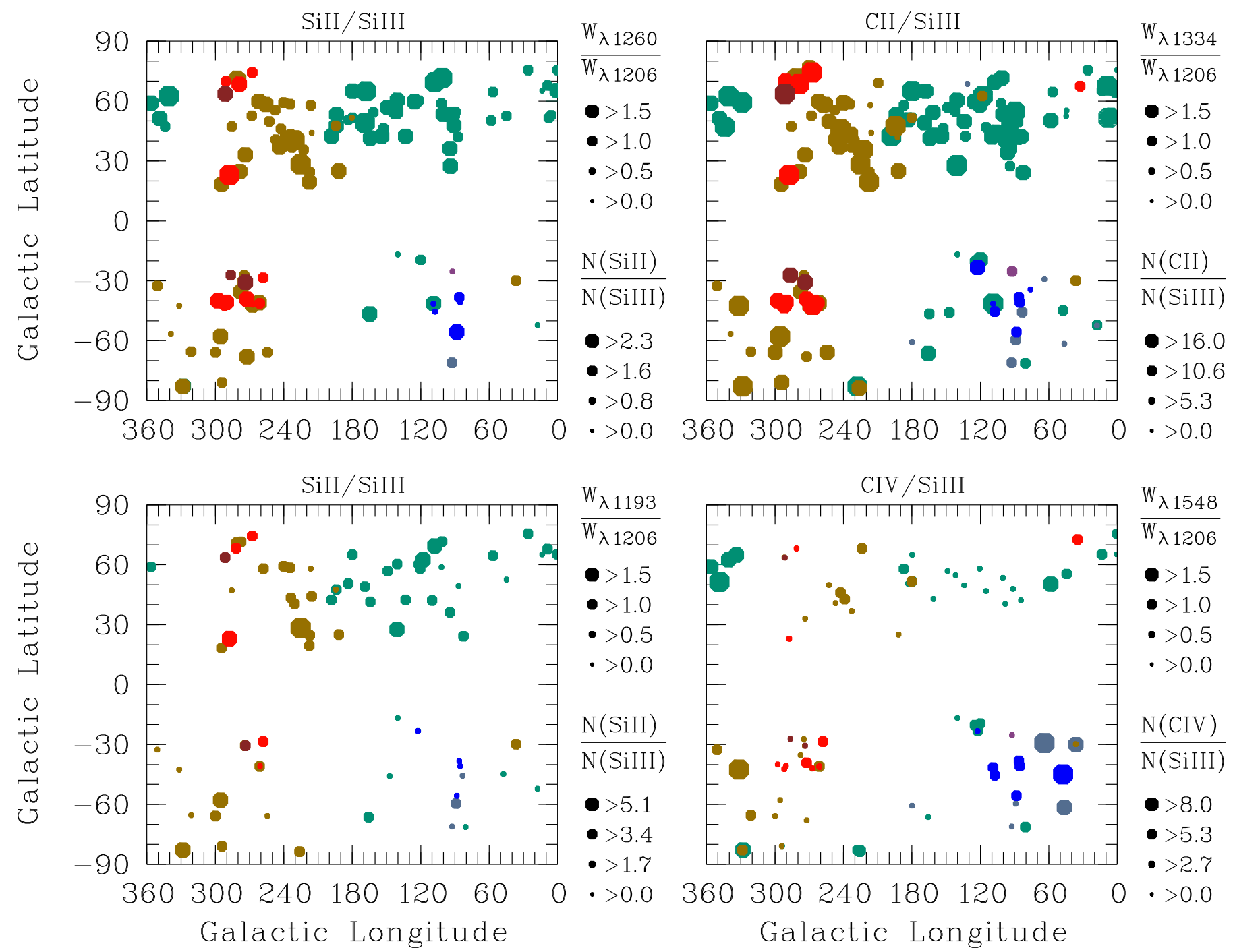

Fig. 7. All-sky distribution of equivalent-width ratios and column-density ratios (assuming optically thin absorption) in high-velocity absorbers (see Sect. 4.2). The color coding indicates the radial velocity of the absorption (see color scheme in Fig. 8).

Galactic center region (see Keeney et al. 2006; Zech et al. 2008; Lehner et al. 2012; Fox et al. 2015).

To statistically compare the $21 \mathrm{~cm}$ properties of HVCfeatures with our UV absorption-line data we have determined the detection rate of $21 \mathrm{~cm}$ emission along the 270 COS sightlines. Out of the 187 high-velocity absorption components detected in Si III absorption, only 46 show associated H I emission in the $21 \mathrm{~cm}$ data with $N(\mathrm{H} \mathrm{I}) \geq 5 \times 10^{18} \mathrm{~cm}^{-2}$ (see Fig. 1, middle column, for an example where high-velocity $\mathrm{HI}$ is detected and aligned with the UV absorption). This translates into an $\mathrm{H} \mathrm{I}$ covering fraction of 17 percent for $\log N(\mathrm{HI}) \geq 18.7$, which is a factor of $\sim 4-5$ lower than the covering fraction for Si III at $\log N($ Si III $) \geq 12.1$. These numbers further demonstrate that the neutral HVCs, as seen in $21 \mathrm{~cm}$ emission, just display the tips of the icebergs of the Milky Way CGM, namely the regions with the highest gas densities, while the bulk of the CGM structures has $\mathrm{H}$ I column densities below $5 \times 10^{18} \mathrm{~cm}^{-2}$ and thus remains invisible in our $21 \mathrm{~cm}$ data (see also Lehner et al. 2012; Richter et al. 2005, 2009; Fox et al. 2006, 2014). Note that the highvelocity H I covering fraction increases to $\sim 30$ percent for log $N(\mathrm{H} \mathrm{I}) \geq 17.9$, if more sensitive $21 \mathrm{~cm}$ data is considered (see Wakker 2004; Lockman et al. 2002).

\subsection{Interpretation of observed trends}

It has been demonstrated in many previous studies that the equivalent-width/column-density ratios of low, intermediate, and high ions represent powerful diagnostic tools to study the ionization conditions in the CGM (e.g., Fox et al. 2014; Werk et al. 2013; Richter et al. 2016). At higher gas densities, recombination is more efficient, and therefore a high Si II/Si III ratio indicates halo gas at relatively high gas densities (same for C II/Si III). Smaller Si II/Si III and C II/Si III ratios, in contrast, indicate regions with high ionization fractions at lower gas densities.

In the northern sky, the observed equivalent widths ratios (Fig. 7) indicate a spatially complex, multi-phase structure of the absorbing gas with many regions that appear to have relatively high densities (with low ions dominating the absorption). Some (but not all) of the northern sightlines with high Si II/Si III ratios are associated with prominent $21 \mathrm{~cm}$ HVC complexes. In the southern sky, the $\mathrm{Si}$ II $\lambda 1260.4 / \mathrm{Si}$ III $\lambda 1206.5$ equivalent ratios $>1$ are predominantly related with relatively dense gas from the neutral gas body of the MS. In the Appendix (Sect. A.2; Fig. A.2) we further discuss the equivalent ratios shown in Fig. 7 as a function of radial velocity. 




Fig. 8. Sky distribution of sightlines in our COS sample in an Hammer-Aitoff projection of Galactic coordinates overlaid on a map of $21 \mathrm{~cm}$ emission for $\left|v_{\mathrm{LSR}}\right| \leq 500 \mathrm{~km} \mathrm{~s}^{-1}$ based on the LAB survey (Kalberla et al. 2005; courtesy Tobias Westmeier). The color scheme is similar to that in Fig. 2.

The fact that UV absorption is observed beyond the outer boundaries of the $21 \mathrm{~cm}$ HVCs at similar radial velocities than the $21 \mathrm{~cm}$ emission (Fig. 8) underlines that the neutral gas bodies are surrounded by extended ionized gas layers that build an interface between the neutral HVCs and the ambient hot coronal gas (e.g., Sembach et al. 2003; Miller \& Bregman 2015). Previous studies have demonstrated that the ionized envelopes of neutral HVCs contain substantially more mass than their neutral cores (Lehner et al. 2012; Shull et al. 2009; Richter et al. 2009; Sect.6). A detailed analysis of UV absorption lines that trace the extended ionized gaseous envelopes of $21 \mathrm{~cm}$ features from the MS recently has been presented by Fox et al. (2014) using a subset of the COS data sample considered here.

As discussed in Sect. 4.3, not all high-velocity UV absorbers are related to known $21 \mathrm{~cm}$ HVCs and the trends presented in Sect. 4.2 indicate the presence of ionized gas streams in the halo with low neutral gas columns. With its low Si II/Si III and high $\mathrm{C}$ IV/Si III ratios, the region $\left(b<0^{\circ}, l<180^{\circ}\right)$ is distinct in its ionization properties from any other large-scale structure in the high-velocity sky (Fig. 7). It apparently contains mostly moderately to highly ionized gas at low gas densities (resulting in low recombination rates). A high degree of ionization is also indicated by the presence of strong high-velocity $\mathrm{O}$ VI absorption in this direction (Sembach et al. 2003). Sembach et al. (1999) modeled the ionization conditions of the high-velocity gas towards PKS 2155-304 and Mrk 509 (see Tables A.1 and A.2) and derived very low thermal gas pressures of $P / k<5 \mathrm{~K} \mathrm{~cm}^{-3}$. Gas at such low pressures would not survive long in the inner halo regions of Milky-Way size galaxies at $r \leq 50 \mathrm{kpc}$, where the gas pressures are expected to be at least one order of magnitude higher (Wolfire et al. 1995). From the density and temperature constraints of the Milky Way's hot coronal gas follows that the thermal gas pressure is expected to decline with radius as $P / k \propto r^{-1.58}$ (Miller \& Bregman 2015), indicating that $P / k \approx$ $20 \mathrm{~K} \mathrm{~cm}^{-3}$ at $r=100 \mathrm{kpc}$ and $P / k \approx 10 \mathrm{~K} \mathrm{~cm}^{-3}$ at $r=200 \mathrm{kpc}$, the latter being the Milky Way's assumed virial radius (Dehnen et al. 2006; McMillan 2011). Similar pressure gradients are also found in numerical simulations of hot gas around Milky Waytype galaxies (N14). The pressure limit of $P / k<5 \mathrm{~K} \mathrm{~cm}^{-3}$ for the high-velocity gas towards PKS 2155-304 and Mrk 509 therefore implies that this absorber is located at very large distances to the disk $(r>250 \mathrm{kpc})$ and possibly traces diffuse gaseous material outside the MW virial radius in the $L G$ in the general direction of the LG barycenter at $l=147^{\circ}, b=-25^{\circ}$ (see Sembach et al. 1999, 2003).

Further support for this scenario comes from the fact that the HVC absorbers in this region exhibit the highest radial velocities in our survey (extreme negative velocities; see Fig. 4, upper left panel and Sect.3.4). Hydrodynamical simulations and observations indicate that the infall velocities of gas at large radii are higher than those close to the disk (at $<5 \mathrm{kpc}$ ), where the kinematics is predominantly determined by the hydrodynamical interaction and the reprocessing of infalling gas by the surrounding hot corona (see Richter 2017, and references therein). We further explore a LG origin of these HVC absorbers in Sect. 7, where we study the gas flow in the LG using constrained cosmological simulations.

\section{Structural properties of the Milky Way CGM}

\subsection{Identification of HVC complexes}

The HVC $21 \mathrm{~cm}$ sky historically has been divided into a large number of apparently coherent structures, the so-called HVC 
Table 3. HVC complexes.

\begin{tabular}{|c|c|c|c|c|c|c|c|c|c|c|c|c|}
\hline HVC complex & $\begin{array}{c}l_{\min } \\
{\left[{ }^{\circ}\right]}\end{array}$ & $\begin{array}{r}l_{\max } \\
{\left[{ }^{\circ}\right]}\end{array}$ & $\begin{array}{r}b_{\min } \\
{\left[^{\circ}\right]}\end{array}$ & $\begin{array}{r}b_{\max } \\
{\left[{ }^{\circ}\right]}\end{array}$ & $\begin{array}{r}v_{\min } \\
{\left[\mathrm{km} \mathrm{s}^{-1}\right]}\end{array}$ & $\begin{array}{r}v_{\max } \\
{\left[\mathrm{km} \mathrm{s}^{-1}\right]}\end{array}$ & $\begin{array}{r}d \\
{[\mathrm{kpc}]}\end{array}$ & $\begin{array}{r}r_{\mathrm{s}}{ }^{a} \\
{[\mathrm{kpc}]}\end{array}$ & $f_{4 \pi}$ & Det. rate & $\begin{array}{l}M_{\mathrm{gas}} \mathrm{c} \\
{\left[M_{\odot}\right]}\end{array}$ & $\begin{array}{c}\mathrm{d} M_{\mathrm{gas}} / \mathrm{d} t^{b} \\
{\left[M_{\odot} \mathrm{yr}^{-1}\right]}\end{array}$ \\
\hline Complex GCN & 0 & 60 & -60 & -30 & -350 & -100 & $\ldots$ & 5 & 0.028 & $2 / 2$ & $1.9 \times 10^{6}$ & 0.039 \\
\hline Complex GCP (Smith Cloud) & 0 & 60 & -30 & 0 & 80 & 200 & 12 & 12 & 0.028 & $1 / 1$ & $2.7 \times 10^{6}$ & 0.027 \\
\hline \multirow[t]{5}{*}{ Magellanic Stream (MS) } & 0 & 180 & -90 & -60 & -500 & -80 & $20-100$ & 55 & 0.333 & $41 / 46$ & $3.0 \times 10^{9}$ & 5.593 \\
\hline & 60 & 120 & -60 & 0 & -500 & -80 & & & & & & \\
\hline & 180 & 360 & -90 & -60 & 80 & 500 & & & & & & \\
\hline & 210 & 360 & -60 & -30 & 150 & 500 & & & & & & \\
\hline & 210 & 300 & -30 & 0 & 150 & 500 & & & & & & \\
\hline \multirow[t]{2}{*}{ Leading Arm (LA) of MS } & 300 & 360 & -30 & 0 & 150 & 500 & $20-100$ & 55 & 0.139 & $8 / 10$ & (see MS) & (see MS) \\
\hline & 240 & 360 & 0 & 60 & 150 & 500 & & & & & & \\
\hline \multirow[t]{3}{*}{ Complex C } & 40 & 90 & 15 & 45 & -250 & -80 & 10 & 10 & 0.062 & $31 / 32$ & $2.7 \times 10^{7}$ & 0.277 \\
\hline & 60 & 110 & 25 & 65 & -250 & -80 & & & & & & \\
\hline & 110 & 150 & 35 & 65 & -250 & -80 & & & & & & \\
\hline \multirow{2}{*}{ Outer Arm (OA) } & 45 & 90 & 0 & 15 & -150 & -80 & 5 & 5 & 0.013 & $\ldots$ & $\ldots$ & \\
\hline & 90 & 160 & 0 & 20 & -150 & -80 & & & & & & \\
\hline Complex G & 60 & 105 & -20 & 0 & -150 & -80 & $\ldots$ & 5 & 0.014 & $\ldots$ & & \\
\hline Complex H & 105 & 150 & -20 & 20 & -250 & -80 & $\geq 5$ & 5 & 0.028 & $2 / 2$ & $9.0 \times 10^{5}$ & 0.018 \\
\hline Complex M & 120 & 200 & 45 & 75 & -150 & -80 & 3 & 3 & 0.037 & $12 / 24$ & $1.6 \times 10^{5}$ & 0.006 \\
\hline \multirow[t]{2}{*}{ Complex A } & 145 & 175 & 30 & 45 & -250 & -140 & $8-10$ & 9 & 0.010 & $5 / 6$ & $2.9 \times 10^{6}$ & 0.033 \\
\hline & 130 & 145 & 20 & 35 & -250 & -140 & & & & & & \\
\hline Anti-Center (AC) & 130 & 200 & 0 & -60 & -500 & -80 & $3-8$ & 5 & 0.065 & $2 / 6$ & $7.7 \times 10^{5}$ & 0.016 \\
\hline AC Shell + ACO & 160 & 210 & -20 & 20 & -130 & -80 & 1 & 1 & 0.031 & -10 & $\ldots$ & \\
\hline Complex WE & 290 & 340 & -30 & 0 & 80 & 150 & $\leq 12$ & 5 & 0.023 & $0 / 1$ & $\ldots$ & \\
\hline Complex WA + WB & 210 & 260 & 0 & 60 & 80 & 200 & $8-20$ & 10 & 0.046 & $21 / 25$ & $7.4 \times 10^{6}$ & 0.075 \\
\hline HVC toward LMC & 275 & 285 & -38 & -28 & 80 & 150 & $\leq 13$ & 9 & 0.002 & & & \\
\hline Complex L & 300 & 360 & 20 & 60 & -200 & -80 & $0.5-1.5$ & 1 & 0.037 & $5 / 7$ & $1.3 \times 10^{4}$ & 0.001 \\
\hline
\end{tabular}

Notes. ${ }^{(a)}$ HVC information compiled from various sources including Wakker (2001, 2003); Wakker et al. (2007, 2008); Thom et al. (2006, 2008); Richter (2006); Richter et al. (2015); Wakker et al. (in prep.). ${ }^{(b)}$ Distance assumed in our model to calculate the total HVC mass and accretion rate (Sect. 6). ${ }^{(c)}$ See Sect. 6.2.

"complexes", primarily to distinguish between gas clouds in the Milky Way halo that are spatially unrelated and may have different origins (Wakker \& van Woerden 1997; Richter 2005, 2017; Putman et al. 2012). The definition of these HVC complexes is based on the position in $l$ and $b$ of the detected $21 \mathrm{~cm}$ features and the radial velocity in which they are observed (see, e.g., Wakker \& van Woerden 1997; Wakker 2001).

In Table 3 we summarize the definition of the most prominent Galactic HVCs, based on the HVC compilation paper of Wakker (2001). For each HVC we list the relevant ranges in Galactic longitude, Galactic latitude, and LSR velocity in the first seven columns. We also list available distance information for each HVC, the sky covering fraction, $f_{4 \pi}$ (based on the angular definition in $l$ and $b$ ), and the detection rate of absorption within the limits in $l, b$, and $v$ from our survey. In the last two rows of Table 3 we further give for each HVC complex the estimated total gas mass and contribution to the CGM gas-accretion rate, as calculated in Sect. 6.

Assuming the $(l, b)$ ranges listed in Table 3 , the MS together with the Leading Arm, LA, and its extended gaseous environment, spans over a total solid angle of $\sim 20000 \mathrm{deg}^{2}$. This gigantic stream of gas therefore represents the by far largest HVC complex, covering almost half of the entire sky. Note that our definition of the angular extent of the MS+LA is different from that used in our previous surveys. This is because we use an angular grid together with an absorption-selected (in $l, b, v_{\mathrm{LSR}}$ ) sightline selection to define the HVC boundaries in our sample, while other studies define the area of the MS+LA based on the $21 \mathrm{~cm}$ contours (e.g., Fox et al. 2014). This aspect needs to be taken into account when comparing the HVC mass- and accretion-rate estimates from different surveys (see review by Richter 2017).

For the MS+LA there are 56 sightlines available in our sample. For a detailed analysis of MS absorption see Fox et al. (2013, 2014). Other HVC complexes with more than 15 spectra are Complex C (32 sightlines), Complex M (24 sightlines) and Complex WA+WB (25 sightlines).

In this study, we do not further investigate the chemical composition of the individual HVC complexes or their internal kinematics. These aspects will be presented in a forthcoming paper. Some interesting information on the multi-phase nature of the various HVCs can be obtained, however, by systematically studying the equivalent-widths/column-density ratios for those HVC complexes, for which good spatial coverage in our COS data set is available.

\subsection{Ion ratios in HVC complexes}

In Sect. 4.4 we have discussed the equivalent-width/columndensity ratios in our high-velocity absorbers in the context of the ionization structure in the clouds. Figure 7 indicates that our large data sample allows us to further investigate this aspect for a limited number of individual HVC complexes, for which sufficient spatial coverage is available.

In Fig. 9 we show the distribution of measured equivalentwidth/column-density ratios of Si II $\lambda 1260.4 / \mathrm{Si}$ III $\lambda 1206.5$ in the MS, Complex C, and Complex WA+WB. Lower values for $\mathrm{Si}$ II/Si III indicate lower-density gas with a high degree of ionization (see Sect. 4.4). For Complex C (100 percent) and Complex WA+WB (85 percent) the majority of the column-density ratios are $>1.0$. In contrast, the MS exhibits quite a number 

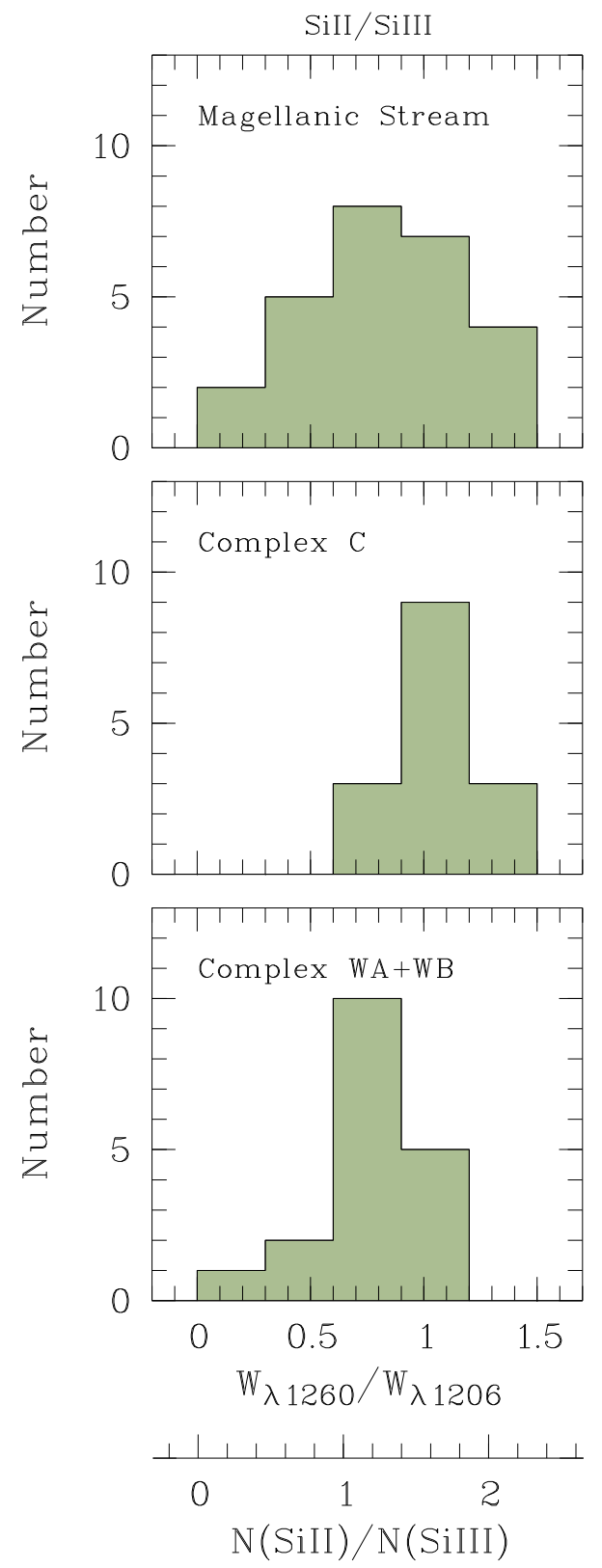

Fig. 9. Distribution of equivalent width ratios of Si II $\lambda 1260.4 /$ Si III $\lambda 1206.5$ in three different HVC complexes.

of absorbers (30 percent) with $\mathrm{Si}$ II/Si III column-density ratios $<1.0$. This trend indicates that the fraction of diffuse ionized gas is larger in the MS than in the Complexes C and WA+WB. One the one hand, this may be partly related to the ab-initio definition of the HVC complexes and their outer boundaries (Table 3 ). On the other hand, the observed trend in Fig. 9 may be a result of the different distances of these HVCs to the disk, which range from $10 \mathrm{kpc}$ (Complex C), 8-20 kpc (Complex WA+WB), to 20-100 kpc (MS; see Table 3, 8th row). Since the CGM gas pressure is expected to decline with increasing distance, HVCs in the outer halo can carry along ionized envelopes with lower densities compared to clouds in the inner halo. This would imply lower recombination rates in the HVC envelopes at large $d$ and thus lower values for $\mathrm{Si}$ II/Si III therein. In addition, diffuse cloud layers around HVCs may be stripped away more efficiently in the inner halo than at larger distances (Heitsch \& Putman 2009).
While these explanations are speculative, they are supported by the fact that low Si II/Si III column-density ratios are typical for CGM absorber at projected distances $\sim 25-150 \mathrm{kpc}$ around other low-redshift galaxies (Richter et al. 2016; their Fig. 15).

\subsection{Connection to Local Group galaxies}

While the majority of the high-velocity absorption features in our survey are produced by gas that is gravitationally bound to the Milky Way, several sightlines intersect the circumgalactic environment of other LG member galaxies. Thus, some of the observed high-velocity absorption features might be related to these galaxies. Previous absorption-line studies have indeed demonstrated that UV absorption is observable in the extended halos of the most nearby LG members, such as the Magellanic Clouds (e.g., de Boer \& Savage 1980; Lehner \& Howk 2007; Richter et al. 2014) and Andromeda (Rao et al. 2013; Lehner et al. 2015; Barger et al. 2016).

A list of the LG member galaxies is presented in Table A.3. 28 of the listed LG galaxies have radial velocities in the range $\left|v_{\text {LSR }}\right|=100-500 \mathrm{~km} \mathrm{~s}^{-1}$ and are located at $|b| \geq 15^{\circ}$. To investigate the relation between high-velocity absorption and gaseous halos of LG galaxies we have calculated the projected (angular) size of these galaxies. We here concentrate on LG galaxies beyond the virial radius of our own galaxy $\left(R_{\mathrm{vir}, \mathrm{MW}} \approx 200 \mathrm{kpc}\right.$; Dehnen et al. 2006; McMillan 2011), thus excluding very nearby galaxies such as the Magellanic Clouds and other MW satellite galaxies. We also only consider the brightest galaxies with $M_{V}<20$ mag that have the largest angular cross section. For the calculation of $R_{\text {vir }}$ and the angular size we use the information on the galaxies' absolute magnitudes and distances, which we transform into an estimate of their luminosities. We then calculate $R_{\text {vir }}$ from the $R_{\text {vir }} / L$ relation from Stocke et al. (2013), which is based on recent halo matching models (Trenti et al. 2010 ), and then determine the angular size at $2 R_{\text {vir. }}$ In Fig. 10 we show the sky distribution and angular size at $2 R_{\mathrm{vir}}$ of the 19 LG galaxies selected in this manner together with the COS sightlines along which HVC absorption is detected. In the finding charts in Fig. B.2 we have indicated the galaxy velocities for those sightlines that pass within $2 R_{\text {vir }}$ of the selected $19 \mathrm{LG}$ galaxies.

There is an interesting match between the general velocity distribution of the galaxies and the absorption velocities. For $l>180^{\circ}$ all $\mathrm{LG}$ galaxies have positive radial velocities from the perspective of the Sun, while for $l \leq 180^{\circ}$ the radial velocities are negative. This trend reflects the general flow of galaxies in the LG towards the LG barycenter (with the MW and M31 being part of this flow), as concluded from studies of the overall dynamics of the LG members (Peebles et al. 2001, 2011; Whiting 2014). As shown in Whiting (2014; his Fig. 1), the absolute space velocities of the LG galaxies are expected to be larger if they are closer to the barycenter. From the perspective of the Milky Way, LG galaxies that lie in the antipodal direction with respect to the LG barycenter $\left(l=147^{\circ}, b=-25^{\circ}\right)$ thus are expected to lag behind the MW flow speed, thus having positive relative velocities, while galaxies located on the opposite side of the LG barycenter will move towards the Milky Way, thus having negative relative velocities. We would like to emphasize that the effect of galactic rotation only plays a minor role for the observed radial velocities at $|b|>30^{\circ}$. As we discuss below, our observations as well as constrained LG simulations suggest that the LG gas (i.e., gas outside the halos of LG member galaxies) is expected to show an identical velocity-flow pattern. 




Fig. 10. Sky distribution of 19 Local Group galaxies that are located outside the virial radius of the MW and that have systemic velocities $\left|v_{\text {sys,LSR }}\right|=100-500 \mathrm{~km} \mathrm{~s}^{-1},|b| \geq 15^{\circ}$, and $M_{V}>20$. The size of the circles indicate the size of their halos at $2 R_{\mathrm{vir}}$ and the color indicates their radial velocity (see color scale). The filled small boxes indicate the position of the COS sightlines with HVC detections using same color coding for the mean absorption velocities.

Figure 10 implies that there are only two general directions in the sky for which the distribution of COS sightlines allows us to systematically study the connection between high-velocity absorption and nearby LG galaxies based on multiple lines of sight.

In the northern sky there are 12 sightlines that pass through the Leo I dwarf galaxy $\left(l=226.0^{\circ}, b=+49.1^{\circ}\right)$ at impact parameters $\rho \leq 2 R_{\text {vir. }}$. High-velocity absorption is detected in this direction at positive velocities in the range +100 to $+250 \mathrm{~km} \mathrm{~s}^{-1}$ (Complex WA), but not near the systemic velocity of the Leo I at $v_{\mathrm{LSR}} \approx 285 \mathrm{~km} \mathrm{~s}^{-1}$. Thus, there is no evidence for an extended gaseous halo around Leo I that could be detected in the ionization states discussed here, supporting earlier results presented by Bowen et al. (1997) based on low-resolution spectra of three sightlines passing the inner halo of Leo I. The lack of absorption does not necessarily mean a lack of CGM gas around Leo I. Since the stellar metallicity of Leo I is low ( 0.05 solar; Bosler et al. 2007), the metal content of the CGM might be too small to be detectable in C IV and Si III within such a small dark matter halo. Alternatively, the gas might be highly ionized and thus invisible in the UV lines traced by COS. In any case, there is no evidence for the presence of cold streams that could feed Leo I with fresh material to form stars. This is not surprising, however, because it is known from HST observations that the star-formation activity in Leo I dropped dramatically 1 Gyr ago (Gallart et al. 1999). Possibly, the lack of cold gas around Leo I and the quenching of star-formation in this dwarf galaxy are related.

In the southern sky, M31 and its companion galaxies are distributed at $l<150^{\circ}$, all of them having negative radial velocities. Figure 10 shows that there are seven sightlines that pass M31 within $2 R_{\text {vir }}$ and that show absorption at high negative velocities. The interpretation of blue-shifted absorption along these sightlines is tricky, however, as there are large amounts of gas from the MS at high negative velocities in the general direction of M31. Disentangling these two components (MS and
M31) can only be done based on specific assumptions about the kinematics of the M31 CGM and the velocity distribution of MS gas. Lehner et al. (2015, hereafter L15) present a detailed analysis of the COS spectra in the direction of M31. They came to the conclusion that the M31 CGM is visible in the spectra of HS 0058+4213, HS 0033+4300, RX J0048.3+3941, UGC 12163, 3C 66A, MRK 335, PG 0003+158, and NGC 7469 in the LSR velocity range between -300 and $-150 \mathrm{~km} \mathrm{~s}^{-1}$, while material from the MS is seen predominantly at $v_{\mathrm{LSR}} \leq$ $-400 \mathrm{~km} \mathrm{~s}^{-1}$. Since we are using the same data in our study, the corresponding absorption features associated with the M31 CGM are visible in the velocity plots in Fig. B.2. Assuming that the L15 velocity model for the MS and M31 is correct, the COS data for the above mentioned sightlines indeed suggest that M31 is surrounded by a massive circumgalactic envelope of gas. Additional COS data for QSO sightlines that are located within $2 R_{\text {vir }}$ will help to distentangle gaseous material stemming from the M31 CGM and the MS (AMIGA project; Lehner et al., in prep.).

There are other, individual sightlines in our COS sample that pass the halos of other LG dwarf/satellite galaxies at $\rho \leq 2 R_{\mathrm{vir}}$ (see Fig. 10). The sightline towards Mrk 509 passes Aquarius at $\rho \sim 1 R_{\text {vir }}$, but no absorption is found within $140 \mathrm{~km} \mathrm{~s}^{-1}$ of the systemic velocity of Aquarius. Similarly, no absorption is found at $v_{\mathrm{LSR}}>300 \mathrm{~km} \mathrm{~s}^{-1}$ towards the background source NGC 3125 , where circumgalactic gas from the nearby $\left(\rho<1.7 R_{\mathrm{vir}}\right) \mathrm{LG}$ galaxies NGC 3109 and Antila would be expected to be seen. The sightline towards PG1011-040 passes Sextans A at $\rho \sim$ $0.5 R_{\text {vir }}$, but, again, no absorption is found within $160 \mathrm{~km} \mathrm{~s}^{-1}$ of the systemic velocity of Sextans A. In contrast to the previous cases, absorption is found towards LBQS-0107-0235 at $v_{\mathrm{LSR}} \approx-200 \mathrm{~km} \mathrm{~s}^{-1}$, a sightline that passes IC $1613\left(v_{\mathrm{sys}}=\right.$ $-236 \mathrm{~km} \mathrm{~s}^{-1}$ ) at $\rho \sim 1.5 R_{\text {vir }}$. The high-velocity absorption towards LBQS-0107-0235 $\left(l=134^{\circ}, b=-65^{\circ}\right)$ is, however, more likely associated with gas from the MS, which shows absorption (and emission) at similar velocities along other 
sightlines in this general direction of the sky. No firm conclusion about the origin of this (weak) absorption feature and its possible relation to IC 1613 can be made based on this single line of sight.

Summarizing, only for the case of M31 do the COS data provide compelling evidence for the presence of an extended gaseous galaxy halo. For the LG dwarfs/satellites outside the virial radius of the MW there are no compelling hints of a circumgalactic gas component.

\subsection{Local Group gas}

One important aspect of our study is the search for a possible connection of high-velocity UV absorption with gas bound to the Local Group as a whole, gas far away from individual galaxies. A number of previous studies have suggested that part of the observed UV and X-ray absorption of high ions such as O VI, O VII, and $\mathrm{O}$ VIII at $\left|v_{\mathrm{LSR}}\right| \leq 500 \mathrm{~km} \mathrm{~s}^{-1}$ is caused by LG gas outside the virial radius of the Milky Way (e.g., Sembach et al. 2003; Collins et al. 2005; Gupta et al. 2012), gas that may harbor the dominating baryon fraction in the Local Group. A direct proof of this hypothesis would require a reliable distance measurement of the absorbing (ionized) gas in the LG via the bracketing method (e.g., Wakker et al. 2007, 2008), which is extremely challenging due to the lack of suitable background sources.

Absorption-line studies of gas in other galaxy groups, however, do provide compelling evidence for the presence of discrete gas structures in groups outside the virial radii of group member galaxies, structures that give rise to absorption in intermediate and high ions (e.g., Stocke et al. 2014). Yet, the interpretation of individual absorbers in group environments often remains inconclusive (see, e.g., the Dorado group; Richter et al. 2016).

From our absorption-line analysis and the comparison between UV absorption and $21 \mathrm{~cm}$ emission we have identified two distinct regions, at $l>240^{\circ}, b>60^{\circ}$ in the northern sky and at $l<120^{\circ}, b=-60^{\circ}-0^{\circ}$ in the southern sky, for which highvelocity gas with $\left|v_{\mathrm{LSR}}\right|>200 \mathrm{~km} \mathrm{~s}^{-1}$ has distinct properties:

- both regions contain very little $\mathrm{HI}$, as indicated by the lack of large-scale $21 \mathrm{~cm}$ emission (Fig. 8);

- towards these directions the high-velocity O VI absorption is particularly strong (Sembach et al. 2003; Wakker et al. 2003);

- detected H I $21 \mathrm{~cm}$ clumps at $l<120^{\circ}, b=-60^{\circ}-0^{\circ}$ have properties that are unusual compared to other HVCs, as they are lacking cold cloud cores and do not show extended diffuse H I emission (see Winkel et al. 2011);

- in the same region, the C IV/Si III ratio is enhanced compared to other regions, indicating a high degree of ionization (Fig. 7, lower right panel);

- high-velocity gas near $l=36^{\circ}$ and $b=-36^{\circ}$ has a very low thermal gas pressure of $P / k<5 \mathrm{~K} \mathrm{~cm}^{-3}$, incompatible with pressures expected in the inner halo of the Milky Way (Wolfire et al. 1995).

As mentioned in Sect. 4.4 these trends provide evidence that the gas at $l<120^{\circ}, b=-60^{\circ}-0^{\circ}, v_{\mathrm{LSR}}<-300 \mathrm{~km} \mathrm{~s}^{-1}$ and possibly also the gas $l>240^{\circ}, b>60^{\circ}, v_{\mathrm{LSR}}>200 \mathrm{~km} \mathrm{~s}^{-1}$ is relatively diffuse and predominantly ionized, possibly residing at large distances from the disk in outer halo or even in the LG (see dicussions in Sembach et al. 1999, 2003; Wakker et al. 2003; Nicastro et al. 2003; Richter et al. 2009; Winkel et al. 2011). Throughout the following, we refer to the absorber regions $l>$ $240^{\circ}, b>60^{\circ}, v_{\mathrm{LSR}}>200 \mathrm{~km} \mathrm{~s}^{-1}$ and $l<120^{\circ}, b=-60^{\circ}-0^{\circ}$,
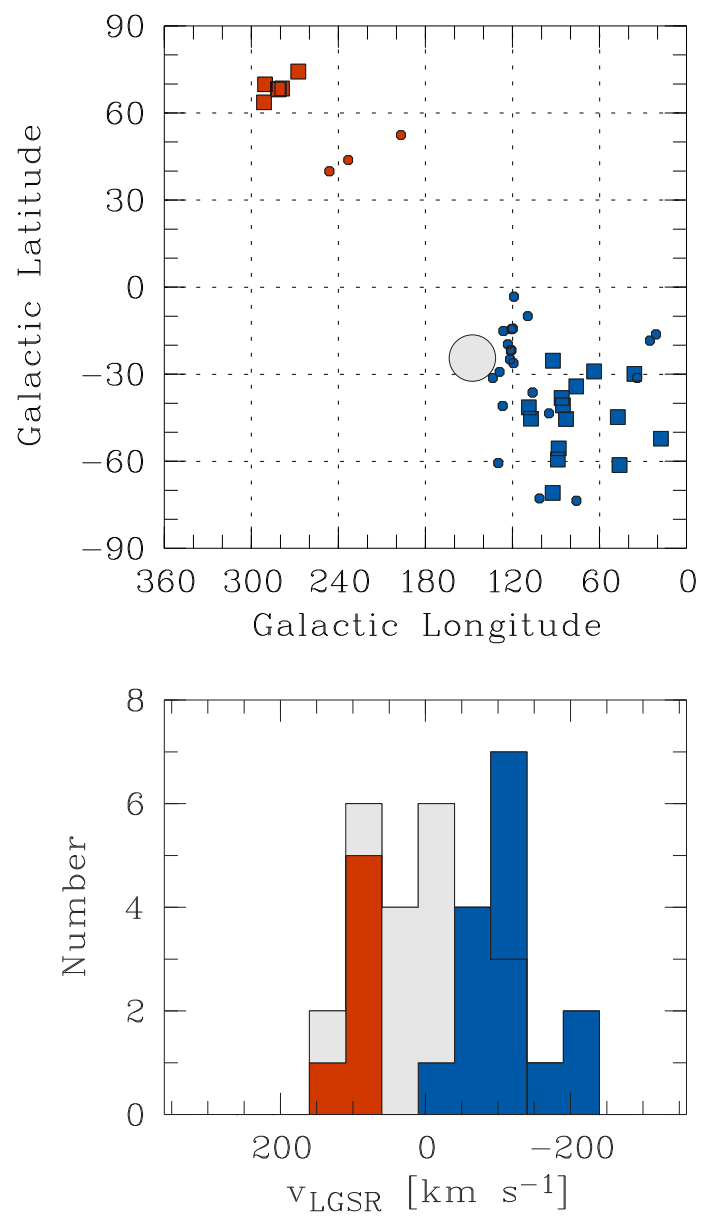

Fig. 11. Upper panel: sky distribution of high-velocity absorbers with $l>180^{\circ}, b>30^{\circ}, v_{\mathrm{LSR}}>200 \mathrm{~km} \mathrm{~s}^{-1}$ and $l<180^{\circ}, b<0^{\circ}$, $v_{\text {LSR }}<-200 \mathrm{~km} \mathrm{~s}^{-1}$ (filled boxes) together with LG galaxies (filled circles) in the same $(l, b)$ range. Lower panel: velocity distribution of these absorbers (colored histograms) and galaxies (gray-shaded histrograms) after transforming their velocities into the LGSR velocity frame.

$v_{\mathrm{LSR}}<-300 \mathrm{~km} \mathrm{~s}^{-1}$ as northern ionized region (NIR) and southern ionized region (SIR), respectively.

In the northern sky, Complex WA is located at $d=8-20 \mathrm{kpc}$ in a $(l, b)$ range similar as the one given above, but at lower radial velocities $\left(v_{\mathrm{LSR}}<200 \mathrm{~km} \mathrm{~s}^{-1}\right.$; see Table 3$)$. Thus, the NIR is not associated with Complex WA. The southern region at $l<120^{\circ}, b=-60^{\circ}-0^{\circ}$ contains negative-velocity gas from the MS (formally defined for $l>60^{\circ}$ ) and from Complex GCN $\left(l<60^{\circ}\right.$; Table 3$)$. In contrast to the MS, origin and distance of Complex GCN are unknown. Therefore, at least some of the detected high-velocity absorption features in the SIR could be located deep in the LG, as there are no observational data that favor a location within the Milky Way halo.

It is striking that the NIR and SIR (together with other nearby high-velocity absorbers) form a velocity dipole on the sky that mimics the one seen for the LG galaxies (Fig. 10). To further emphasize this, we plot in the upper panel of Fig. 11 the sky distribution of all high-velocity absorbers with $l>180^{\circ}, b>30^{\circ}$, $v_{\mathrm{LSR}}>200 \mathrm{~km} \mathrm{~s}^{-1}$ and $l<180^{\circ}, b<0^{\circ}, v_{\mathrm{LSR}}<-200 \mathrm{~km} \mathrm{~s}^{-1}$ (filled boxes) together with LG galaxies (filled circles) in the same $(l, b)$ range.

LG gas that follows the same flow towards the LG barycenter as the galaxies (Sect. 5.3) would show exactly the kind of velocity dipole that is seen in Fig. 11, upper panel. The Milky 
Way would move faster towards the barycenter than the LG gas that would lag behind in the general anti-barycenter direction, so that positive relative velocities are expected. In the barycenter direction, the Milky Way halo would ram into LG gas that is at rest at the barycenter or that flows to the barycenter from the opposite side of barycenter (i.e., from the direction of M31 and its large-scale environment), so that it would have high negative velocities. Similar arguments already have been used by Blitz et al. (1999) to model the expected kinematics of an extragalactic HVC population (see also Collins et al. 2005).

In the lower panel of Fig. 11 we show the velocity distribution of the NIR and SIR absorbers after transforming their velocities into the Local Group Standard of Rest (LGSR) velocity frame. In general, the absolute velocity spread in the sample of the absorbers is substantially reduced from $\Delta v=720 \mathrm{~km} \mathrm{~s}^{-1}$ in the LSR frame to $\Delta v=360 \mathrm{~km} \mathrm{~s}^{-1}$ in the LGSR frame. Similarly, the standard deviation of the velocity distribution reduces from $260 \mathrm{~km} \mathrm{~s}^{-1}$ in the LSR velocity frame to $110 \mathrm{~km} \mathrm{~s}^{-1}$ in the LGSR frame (see also Sembach et al. 2003; and Nicastro et al. 2003, for identical trends in FUSE O VI absorption-line data). In view of the latitude range, this trend can only partly be related to the removal of the Galactic rotation effect in the LGSR frame.

A more detailed interpretation of this plot is difficult, however, without knowing the contribution of the MS to the velocity distribution at high negative velocities. In addition, the overall kinematics of the LG gas is expected to be more complicated than the kinematics of the galaxies because some of the gas is being accreted by the LG galaxies, thus having different directions of motion. The 3D velocity distribution of the gas might also be influenced by ram-pressure forces and large-scale turbulent flows. To critically evaluate the importance of these processes and to investigate whether a simple flow scenario towards the group barycenter is realistic for LG gas around MW and M31, hydrodynamical simulations of the LG and its gaseous environment are required (N14). In Sect. 7 we analyze such simulations to further study the expected large-scale kinematics of gas in the LG.

\subsection{Spatial clustering and sub-structure}

To characterize the amplitude of spatial clustering of HVC absorption components on the sky we analyze the two-point correlation function, $\xi(\theta, \Delta v)$, of HVC absorbers, where $\theta$ represents the angular distance between any two sightlines and $\Delta v$ is the selected velocity bin. We determined $\xi(\theta, \Delta v)$ in our data set by counting the number of HVC absorption components, $\mathcal{N}_{\mathrm{HVC}}$, in a given bin $\Delta v$ along all sightline pairs in our sample and comparing the result to the number of absorbers derived for a random

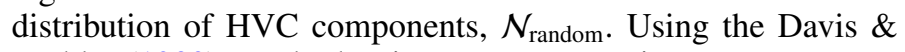
Peebles (1983) standard estimator, we can write

$\xi(\theta, \Delta v)=\frac{\mathcal{N}_{\mathrm{HVC}}(\theta, \Delta v)}{\mathcal{N}_{\text {random }}(\theta, \Delta v)}-1$.

The random HVC absorber distribution was calculated from a Monte-Carlo simulation, in which we simulated 100 realizations of random distributions in $(l, b)$ and $v=$ $[-500,-100][+100,+500]$ along 270 sightlines.

The result of our analysis is shown in Fig. 12, where we plot $\xi(\theta, \Delta v)$ against $\theta$ for all sightline pairs with $\theta \leq 200^{\circ}$. A strong clustering signal is evident for small angular separations $\theta \leq 30^{\circ}$, while $\xi$ decreases rapidly for larger angles. Obviously, the high-velocity absorption is caused by coherent gas structures that typically span $<30^{\circ}$ on the sky (see also Lehner et al. 2012).

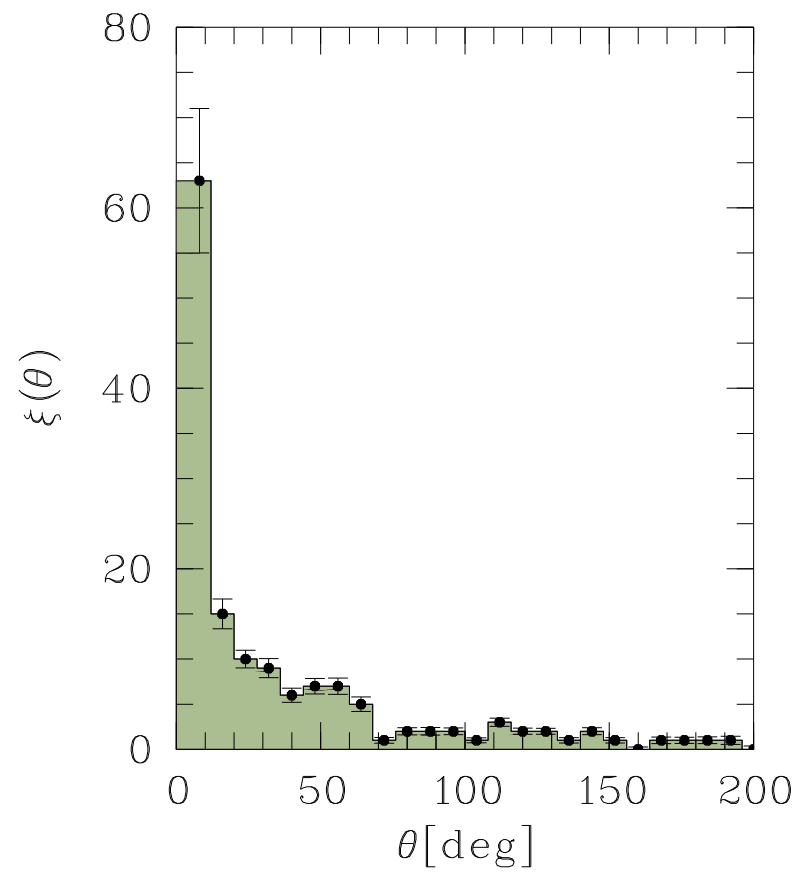

Fig. 12. Two-point correlation function $\xi(\theta, \Delta v)$ of HVC absorbers along sightlines with angular separations of $d \leq 200 \mathrm{deg}$.

This result is in line with the visual appearance of typical angular sizes of the $21 \mathrm{~cm} \mathrm{HVC} \mathrm{complexes} \mathrm{displayed} \mathrm{in} \mathrm{Fig.} \mathrm{8.} \mathrm{At}$ a distance of $d=10 \mathrm{kpc}$ (e.g., Complex C) an angular separation of $\theta=1^{\circ}$ on the sky corresponds to a linear separation of $l \approx 0.0175 d\left(\theta / 1^{\circ}\right)=175 \mathrm{pc}$.

The sky distribution of the sightlines shown in Fig. 2 indicates that there are sightline pairs with small angular separations that can be used to study the internal structure of the Milky Way CGM on pc scales by comparing along two adjacent sightlines the absorption depths and velocity centroids of high-velocity absorbers. We have selected 12 sightline pairs in our COS sample with angular separations $\leq 1^{\circ}$ to identify such differences in the absorption patterns. In Fig. 13 we show three examples of these pairs, where the angular separation between the sightlines is indicated on top of each panel. The first sightline pair in Fig. 13 (RX J0439.6-5311/HE 0435-5304) has an angular separation of only $29^{\prime}$. These sightlines trace gas from the MS at high positive velocities. There are significant differences in the absorption depth of all plotted ion transitions, indicating changes in the gas properties on (relatively large) scales of $l \approx 450 \mathrm{pc}$ if a MS distance of $d=55 \mathrm{kpc}$ is assumed. The sightlines towards Mrk 380 and RXJ 01745+7408 (Fig. 13, middle panel) are separated by $\theta=31^{\prime}$ and pass through negative-velocity gas in HVC complex A at $d \approx 10 \mathrm{kpc}$ distance (van Woerden et al. 1999; Wakker 2001). Significant differences are seen in the singlyionized species Si II and C II, indicating differences in the gas distribution on scales of $l \approx 90 \mathrm{pc}$. The third sightline pair in Fig. 13 (HE 0439-5254/RX J0439.6-5311) again shows differences in the absorption properties in the MS on scales of several hundred pc. The full set of these plots is presented in Fig. B.1. In addition, we discuss in the Appendix (Sect. A.3; Fig. A.3) smallscale structure in the equivalent-widths maps of Complex $\mathrm{C}$ and Complex WA and previous results on small-scale structure in HVCs. 

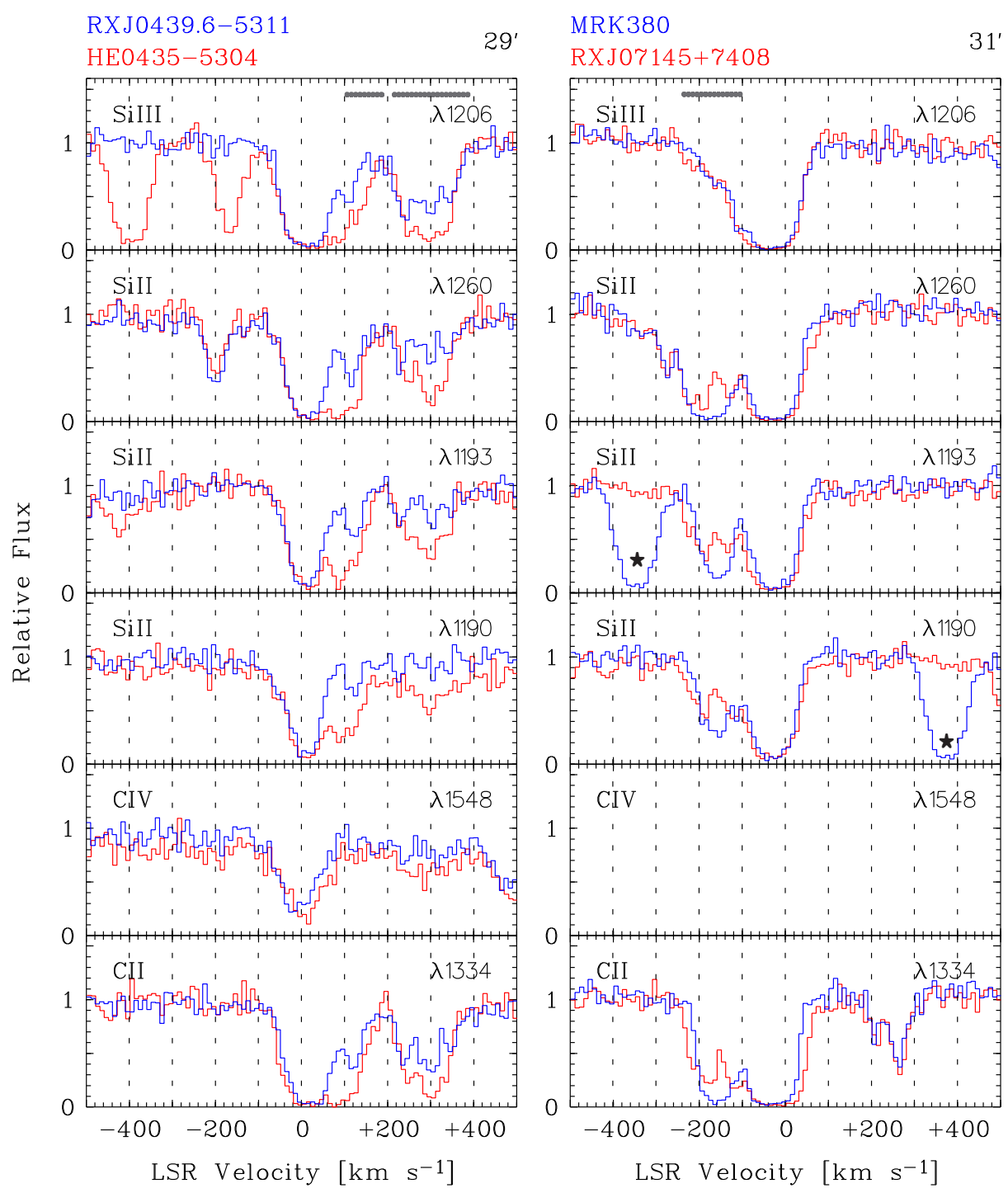

HE0439-5254

RXJ0439.6-5311

$31^{\prime}$

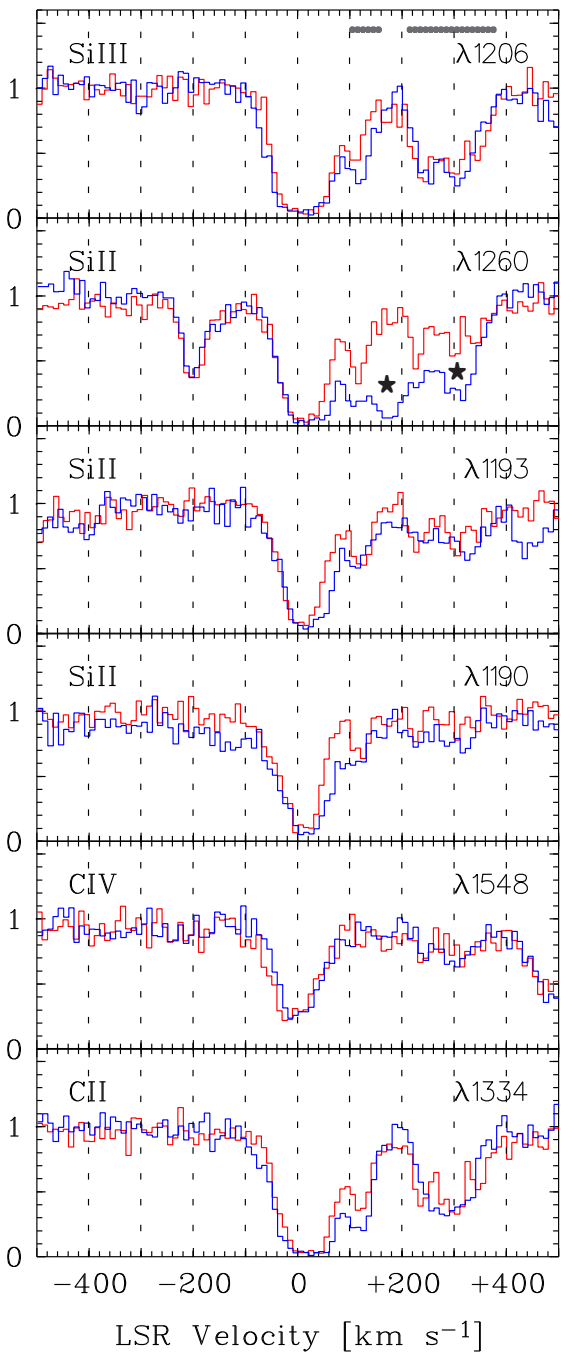

Fig. 13. Three examples for sightline pairs with angular separations $\leq 1^{\circ}$. Differences in the absorption profiles indicate small-scale structure in the absorbing gas. High-velocity absorption components are indicated with the gray bar. Blends with intergalactic absorption features are indicated with a star symbol. QSO names and angular separations between the sightlines are given on top of each panel. Appendix B contains this plot for all 12 sightline-pairs.

The observed variations in the UV absorption patterns of HVCs along adjacent sightlines further support the scenario, in which HVCs represent coherent gaseous structures with largescale kinematics (on kpc scales) and small-scale variations (on pc and sub-pc scales; see Sect. A.3) in physical conditions.

\section{Mass estimate of the Milky Way's CGM}

\subsection{Total gas columns and ionization fractions}

A commonly used approach to study the ionization conditions in individual high-velocity clouds in the Milky Way halo is the use of column density ratios of low, intermediate, and high ions together with Cloudy (Ferland et al. 2013) ionization models (e.g., Richter et al. 2009, 2013; Fox et al. 2013, 2014, 2015; Herenz et al. 2013). Such ionization models provide useful constraints on the gas densities, ionization parameters, and metal abundances in the gas, but they require accurate information on the column densities of different ions, the velocity-component structure, and the distance of the absorbing gas from the Milky Way disk. The latter aspect is important, because the ionizing radiation field in the Milky Way halo is expected to be anisotropic with contributions from the overall UV background and from stars in the Milky Way disk (see Fox et al. 2005).

Because our COS absorber sample is limited in $\mathrm{S} / \mathrm{N}$, spectral resolution, and because model-constraining distance information is available for only a sub-set of the HVC complexes, we refrain from using Cloudy models in this all-sky survey, as these would be afflicted with large systematic uncertainties. Instead, we estimate for each high-velocity absorber a lower limit for the total column density of hydrogen, $N(\mathrm{H})=N(\mathrm{H} \mathrm{I})+N(\mathrm{H}$ II $)$, from the column densities of $\mathrm{Si}(\mathrm{Si}$ II+Si III) and $\mathrm{C}$ (C II+C IV) and combine the values for $N(\mathrm{H})$ with the neutral gas column densities derived from the $21 \mathrm{~cm}$ data (see also Fox et al. 2014). To obtain a limit for $N(\mathrm{H})$ we define

$\log N^{\prime}(\mathrm{H})=\log \left[\frac{N(\mathrm{Si} \mathrm{II})+N(\operatorname{Si} \mathrm{III})}{X(\mathrm{Si} / \mathrm{H})_{\odot}}\right]$ 


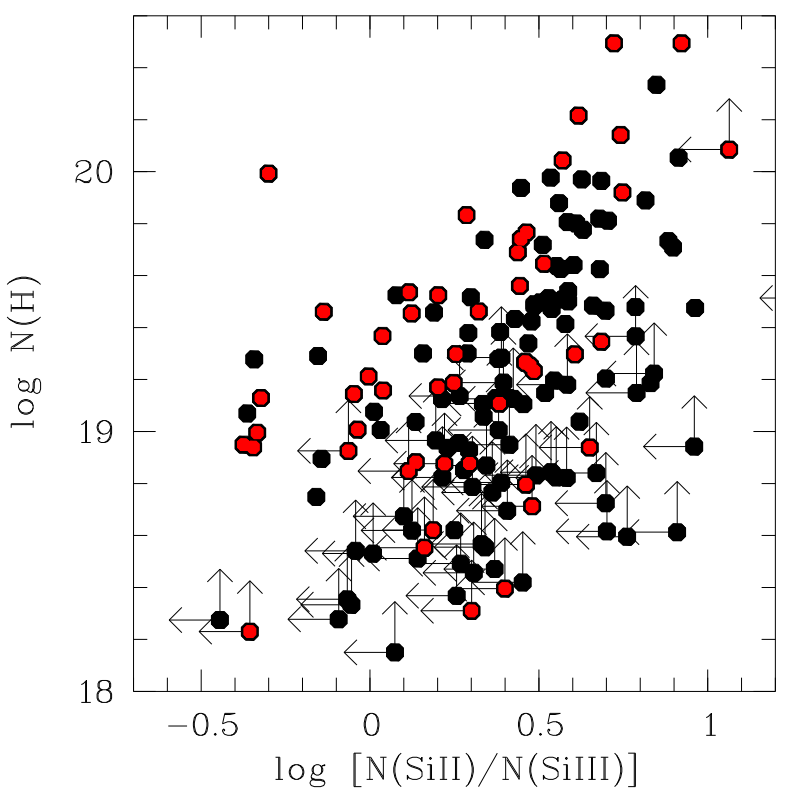

Fig. 14. Scatter plot showing the logarithmic total hydrogen column density for the $187 \mathrm{HVC}$ absorbers plotted against the logarithmic Si II/Si III column-density ratio. Values for MS sightlines are indicated in red.

and

$\log N^{\prime \prime}(\mathrm{H})=\log \left[\frac{N(\mathrm{C} \mathrm{II})+N(\mathrm{C} \mathrm{IV})}{X(\mathrm{C} / \mathrm{H})_{\odot}}\right]$

and consider the larger of the two values for each absorber; in 90 percent, $N^{\prime}(\mathrm{H})>N^{\prime \prime}(\mathrm{H})$.

In the above equations, $(\mathrm{Si} / \mathrm{H})_{\odot}=3.24 \times 10^{-5}$ and $(\mathrm{C} / \mathrm{H})_{\odot}=$ $2.69 \times 10^{-4}$ are the solar abundances of $\mathrm{Si}$ and $\mathrm{C}$ (Asplund et al. 2009). The parameter $X$ denotes the mean metallicity of the gas in solar units. We assume $X=0.1$, motivated by the fact that most of the prominent HVCs in the Milky Way halo, such as the MS, Complex C, Complex A, have $\alpha$ abundances of $\sim 10$ percent solar (Wakker et al. 1999; Richter et al. 2001; Sembach et al. 2004; Fox et al. 2013, 2015).

We need to emphasize at this point that the values for $N^{\prime}(\mathrm{H})$ and $N^{\prime \prime}(\mathrm{H})$ derived in this way reflect only the amount of hydrogen that is traced by Si II, Si III, C II, and C IV. Photoionized and shock-heated H II at lower gas densities and/or higher gas temperatures that is traced by higher metal ions such as $\mathrm{O}$ VI, O VII, and O VIII is known to exist in the Milky Way's CGM as well (Sembach et al. 2003; Wakker et al. 2003; Gupta et al. 2012; Miller et al. 2016), but is not sampled in our data. In addition, because the Si II, C II, Si III absorption is saturated in the strongest absorbers, $N^{\prime}(\mathrm{H})$ and $N^{\prime \prime}(\mathrm{H})$ are systematically underestimated in these systems. However, $N^{\prime}(\mathrm{H})$ and $N^{\prime \prime}(\mathrm{H})$ cannot be smaller than $N(\mathrm{HI})$ and thus the H I column density measured from the $21 \mathrm{~cm}$ data sets the lower limit for the total hydrogen column. Consequently, we adopt as final value $N(\mathrm{H})=\operatorname{MAX}[N(\mathrm{HI})$, $\left.N^{\prime}(\mathrm{H}), N^{\prime \prime}(\mathrm{H})\right]$, which represent conservative lower limit for the total hydrogen column density in each absorber.

In Fig. 14, we show the total hydrogen column density plotted against the $\mathrm{Si}$ II/Si III column-density ratio for all 187 highvelocity absorbers, where limits for these parameters are indicated with arrows (red dots indicate values for MS sightlines). Despite the large scatter there is a clear trend of $\log N(\mathrm{H})$ increasing with increasing Si II/Si III ratio. The intrinsic scatter of this distribution would be significantly smaller if the many data points with limits in Si II/Si III and H I would be removed. The observed trend implies that high-column density HVCs (which are also seen in H I $21 \mathrm{~cm}$ emission) have a smaller mass fraction of diffuse gas (traced by Si III) than low-column density HVCs, which are predominantly diffuse and ionized. Although $\mathrm{Si}$ III is the most sensitive $\mathrm{Si}$ ion to trace diffuse, multi-phase gas in HVCs, the dominating ionization state of $\mathrm{Si}$ in our HVC absorber sample (in terms of column density) is Si II. In our sample, the Si II column density exceeds that of Si III for gas columns log $N(\mathrm{H})>10^{19} \mathrm{~cm}^{-2}$, typically.

In Fig. 15, upper panel, we show the distribution of the measured 46 neutral hydrogen column densities $(\log N(\mathrm{HI})>18.7)$ in our HVC sample. The majority ( 87 percent) of the measured H I column densities lie below $10^{20} \mathrm{~cm}^{-2}$ and for $\log N(\mathrm{HI})>$ 19.4 the distribution shows a decline in the form of a power-law, similar to what has been found in previous H I HVC surveys (see, e.g., Lockman et al. 2002; Wakker 2004). In the lower panel of Fig. 15 we display the distribution of the estimated total hydrogen column densities for all $187 \mathrm{HVC}$ absorbers. Also the total hydrogen column densities predominantly are $<10^{20} \mathrm{~cm}^{-2}$ with a median value of $\log \langle N(\mathrm{H})\rangle=19.13$. Because of the observational restrictions in constraining absolute values for $N(\mathrm{HI})$ and $N(\mathrm{H})$ for our HVC sample, only upper limits for the neutral gas fraction $\log f_{\mathrm{HI}}=\log N(\mathrm{HI})-\log N(\mathrm{H})$ can be given for most sightlines. Values/limits for $\log N(\mathrm{H} \mathrm{I}), \log N(\mathrm{H})$, and $\log f_{\mathrm{HI}}$ for each high-velocity absorber in our sample are listed in Table A.4.

\subsection{The total gas masses of HVCs}

To estimate the total gas mass of the entire population of Galactic HVCs we assume that HVCs are gas patches that are ellipsoidally/spherically distributed around the Milky Way disk. Let $r_{\text {disk }}=20 \mathrm{kpc}$ be the fixed radius of the Milky Way H I disk and $r_{\mathrm{s}}$ be the distance of the absorbing structures from the Galactic center. We adopt a spherical distribution of HVCs for $r_{\mathrm{s}} \geq r_{\text {disk }}$ and a ellipsoidal distribution for $r_{\mathrm{s}}<r_{\text {disk }}$ (i.e., in the latter case, the height above the disk is smaller than the disk radius).

For each sphere, the area covered by HVCs at distance $r_{\mathrm{s}}$ then is simply $A\left(r_{\mathrm{s}}\right)=4 \pi r_{\mathrm{s}}^{2} f_{4 \pi}\left(r_{\mathrm{s}}\right) B$, where $f_{4 \pi}\left(r_{\mathrm{s}}\right)$ is the covering fraction of HVC gas on that sphere. The parameter $B$ denotes the correction factor for ellipsoidal symmetry and is defined as $B=1+\left(r_{\mathrm{s}} / r_{\text {disk }}\right)^{2}(\operatorname{arctanh} \epsilon) / \epsilon$ with the ellipticity $\epsilon=\left(1-\left(r_{\mathrm{s}} / r_{\text {disk }}\right)^{2}\right)^{1 / 2}$. Based on our observations, we estimate the mean total hydrogen column density, $N(\mathrm{H})$, for each HVC complex listed in Table 3 using the method described in the previous subsection.

In general, $N(\mathrm{H})$ is the product of the hydrogen volume density and the absorption path length, $N(\mathrm{H})=n_{\mathrm{H}} d$. In our HVC model, $d$ can be regarded as the thickness of the absorbing gas layer. The mass per particle is $\mu m_{\mathrm{H}}$ with $\mu=1.4$ as factor that corrects for the presence of helium and heavy elements in the gas. Combining these equations, we obtain an expression for the gas mass of an $\mathrm{HVC}$ at radius $r_{\mathrm{s}}$ then in the form

$M_{\mathrm{HVC}}\left(r_{\mathrm{s}}\right)=\mu m_{\mathrm{H}} 4 \pi r_{\mathrm{s}}^{2} f_{4 \pi}\left(r_{\mathrm{s}}\right) B\langle N(\mathrm{H})\rangle_{\mathrm{HVC}}$.

To calculate the total gas mass in each HVC, we adopt the values for $r_{\mathrm{s}}$ and $f_{4 \pi}\left(r_{\mathrm{s}}\right)$ listed in Table 3 and take the median total gas column density, $\langle N(\mathrm{H})\rangle$ in each HVC complex. The total gas mass in the CGM traced by the ions in our survey then is obtained by summing over all $M_{\mathrm{HVC}}\left(r_{\mathrm{s}}\right)$ (Table 3, Col. 12).

For the MS (including the LA), for instance, we assume $r_{\mathrm{s}}=$ $55 \mathrm{kpc}$ and $f_{4 \pi}=0.47$ and from our measurements, together with Eqs. (5) and (6), we obtain $M_{\mathrm{MS}}=3.0 \times 10^{9} M_{\odot}$. This value is 

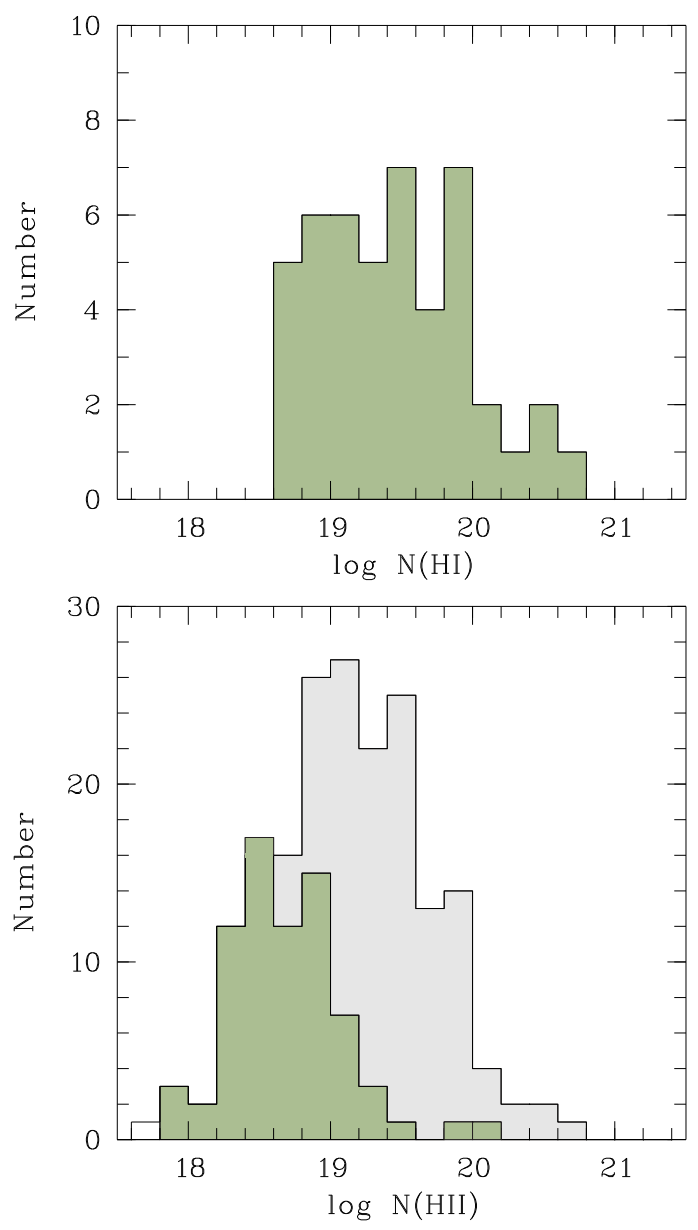

Fig. 15. Upper panel: distribution of the measured neutral hydrogen column densities with $\log N(\mathrm{HI})>18.7$ for $43 \mathrm{HVC}$ absorbers. Lower panel: distribution of total hydrogen column densities. The gray-shaded area shows the distribution of $\log N(\mathrm{H})$ derived for all $179 \mathrm{HVC}$ absorbers. The green-shaded area indicates the distribution of $\log N(\mathrm{H})$ for systems with unsaturated Si II/C II/Si III absorption (see Sect. 6.1).

higher than the one estimated by Fox et al. (2014), which is due to larger assumed sky covering fraction of the MS in our study ( $f_{4 \pi}=0.47$ vs. 0.27 ; see Sect. 3.1 in Fox et al. 2014). The total mass of the MS would be even higher, if some of the gas was located at larger distances than the canonical $55 \mathrm{kpc}$ assumed here. In fact, several authors suggested that the bulk of the MS is located at $d=100 \mathrm{kpc}$ rather than at $d=55 \mathrm{kpc}$ (Besla, et al. 2012; Jin \& Lynden-Bell 2008; Bland-Hawthorn et al. 2013), implying that the MS mass could be as large as $>5 \times 10^{9} M_{\odot}$.

The contribution of all other HVCs to the total mass is small; their gas mass sums up to a total value of no more than $M_{\mathrm{HVC} \text { swithoutMS }}=4.3 \times 10^{7} M_{\odot}$. The total mass of high-velocity gas around the Milky Way thus is clearly dominated by material that stems from the interaction of the Milky Way with the Magellanic Clouds, while the contribution of gas from other origins is negligible ( $\sim 1$ percent; see also Fox et al. 2014). The total mass of HVCs thus is essentially identical to the estimated mass of the MS, $M_{\mathrm{HVC}}=3.0 \times 10^{9} M_{\odot}$, which is $\sim 43$ percent of the ISM gas mass in the Galactic disk $\left(\sim 7 \times 10^{9} M_{\odot}\right.$; Ferriere 2001$)$. Because in our survey we do not account for the highly-ionized gas phase in HVCs as traced, for instance, by O VI (e.g., Sembach et al. 2003; Fox et al. 2004), our estimates for the HVC masses represent conservative lower limits.
For the calculation of the Milky Way's gas-accretion rate of high-velocity gas (Table 2, last column) we set

$\frac{\mathrm{d} M_{\mathrm{HVC}}}{\mathrm{d} t}=\frac{M_{\mathrm{HVC}} \eta v_{\text {infall }}}{r_{\mathrm{s}}}$,

where we assume $v_{\text {infall }}=100 \mathrm{~km} \mathrm{~s}^{-1}$ as characteristic value for the infall velocity of the gas and define $v_{100}=v_{\text {infall }} /\left(100 \mathrm{~km} \mathrm{~s}^{-1}\right)$ as according scaling parameter. The choice of $v_{\text {infall }}=100 \mathrm{~km} \mathrm{~s}^{-1}$ is motivated by the results from hydrodynamical simulations of neutral HVCs infalling into hot, coronal halo gas (Heitsch \& Putman 2009; Joung et al. 2012). These studies demonstrate that the bulk of infalling gas clouds have velocities far below the expected ballistic velocities due to the hydrodynamical interaction between the infalling gas and the ambient hot medium. The parameter $\eta \leq 1$ denotes the fraction of the HVC gas that actually arrives in the Milky Way disk (in whatever form and state). Estimating realistic values for $\eta$ is challenging, as this requires full-fledged hydrodynamical models that describe the passage of neutral and ionized gas through the hot coronal gas of the Milky Way (see again Heitsch \& Putman 2009; Joung et al. 2012). We here consider the range $\eta=0.1-1.0$ as realistic, but carry along this scaling parameter for the following estimates.

The lower limit of the total gas accretion rate from all HVCs listed in Table 3 comes out to $6.1 v_{100} \eta M_{\odot} \mathrm{yr}^{-1}$. Here, the MS contributes with $5.5 v_{100} \eta M_{\odot} \mathrm{yr}^{-1}$, while the contribution of all other HVCs sums up to $0.6 v_{100} \eta M_{\odot} \mathrm{yr}^{-1}$. If much of the MS would be located at $d=100 \mathrm{kpc}$ (as discussed above) or the infall velocity of the gas would be higher, the gas accretion rate would be increased accordingly. For comparison, the current star-formation rate of the Milky Way is estimated to be $S F R(\mathrm{MW}) \leq 1.9 M_{\odot} \mathrm{yr}^{-1}$ (Chomiuk \& Povich 2011; Robitaille $\&$ Whitney 2010). The infall of gas towards the disk in the form of HVCs thus provides a significant mass flow that is expected to substantially influence the future star-formation rate of the Milky Way. Because we consider the velocity range $\left|v_{\mathrm{LSR}}\right|=100-$ $500 \mathrm{~km} \mathrm{~s}^{-1}$ in our analysis, our calculations do not cover the contribution of low-velocity halo clouds (LVHCs; Peek et al. 2009; Zheng et al. 2015) to the total gas mass in the Milky Way halo and to the global gas accretion rate.

If we re-scale $f_{4 \pi}(\mathrm{MS})$ to previously used, smaller skycovering fractions of the Stream, our values for $M_{\mathrm{HVC}}$ and $\mathrm{d} M_{\mathrm{HVC}} / \mathrm{d} t$ are in good agreement with our previous results (Fox et al. 2014) and with estimates from earlier studies (e.g., Putman et al. 2012; Richter 2012; Lehner \& Howk 2011). We would like to note that these previous estimates are based on other data sets and different model assumptions, so that a direct comparison remains difficult. For a detailed discussion of observational and theoretical studies of the Milky Way's gas accretion we refer to the review by Richter (2017).

\section{The Milky Way's CGM in a cosmological context}

\subsection{A search for antipodal sightlines}

With 270 more or less randomly distributed sightlines at hand, it is promising to search for sightline pairs in our COS data sample that represent antipodes on the celestial sphere. A systematic study of antipodal sightlines and their spectra allows us to produce composite spectra that reflect the absorption characteristics of complete sightlines through the Milky Way disk and halo environment at the position of the Sun from different external vantage points (see Marggraf \& de Boer 2000, for an example towards LMC/M81). Such antipodal composite absorption spectra then can be readily compared with spectra of intervening 

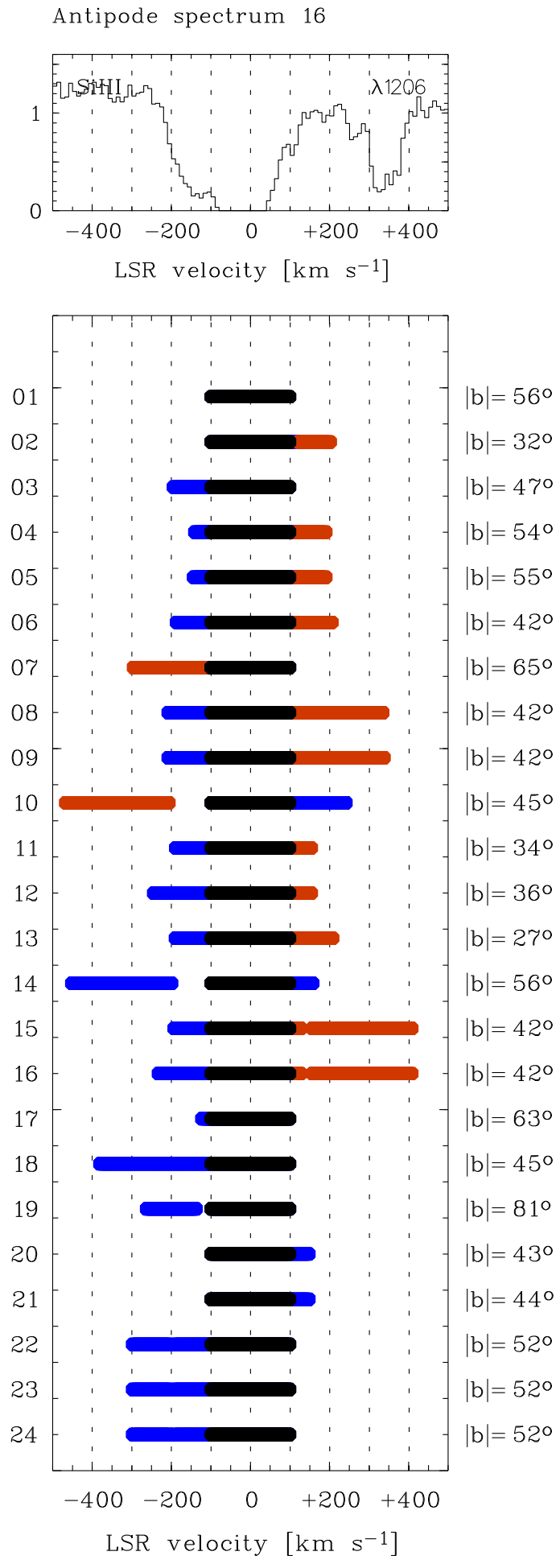

Fig. 16. Upper panel: example for a composite absorption profile from two antipodal sightlines (from antipode pair No. 16). Lower panel: velocity distribution of the $\mathrm{Si}$ III $\lambda 1206.50$ absorption in 24 antipodal composite profiles with disk gas $\left(\left|v_{\mathrm{LSR}}\right| \leq 100 \mathrm{~km} \mathrm{~s}^{-1}\right)$ indicated in black and CGM absorption $\left(\left|v_{\mathrm{LSR}}\right|>100 \mathrm{~km} \mathrm{~s}^{-1}\right)$ indicated in red (MS absorption) and blue (other HVCs).

absorption-line systems, such as DLAs. They provide an idea of how the Milky Way and its gaseous environment would look like if it was seen as a QSO absorption-line system from far away (Herenz et al. 2013).
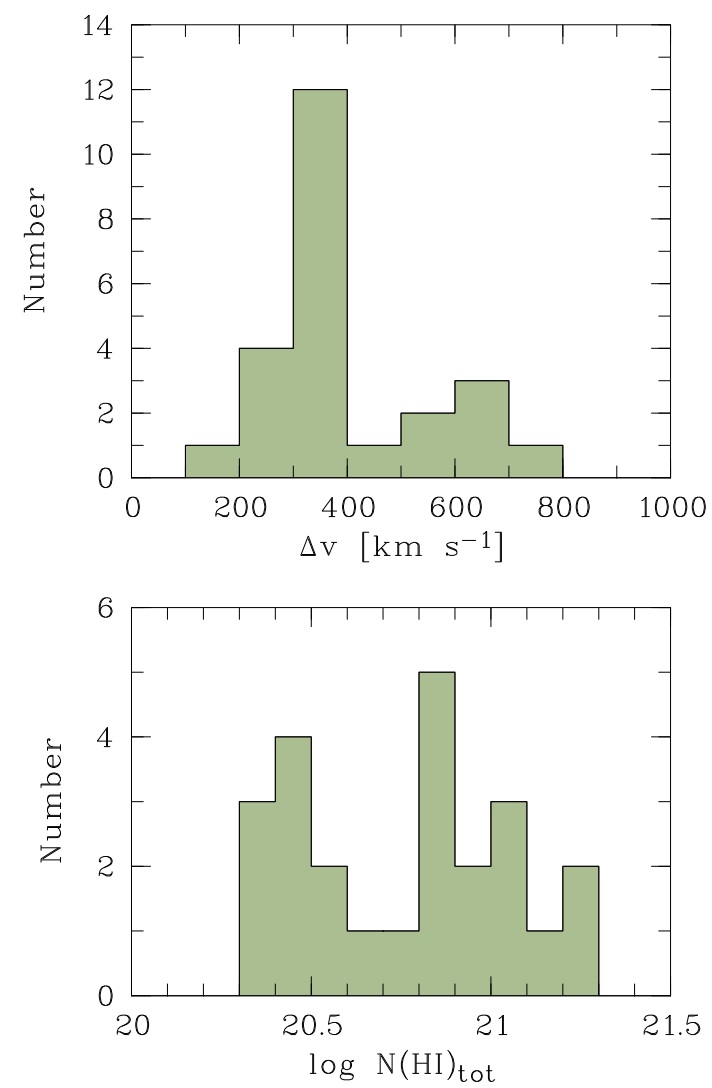

Fig. 17. Upper panel: distribution of velocity widths ( $\Delta v$, in bins of $100 \mathrm{~km} \mathrm{~s}^{-1}$ ) in 24 antipodal composite spectra sampling Milky Way disk and halo gas. Lower panel: distribution of total H I column densities along these 24 sightline-pairs derived from $21 \mathrm{~cm}$ data.

We consider antipodal sightline pairs in cones with opening angles of $\theta=6^{\circ}$ in our sample and find 24 such pairs (48 QSO spectra); they are listed in Table A.5. The basic concept of identifying antipodal QSO sightlines at $\left(l_{\mathrm{A}}, b_{\mathrm{A}}\right),\left(l_{\mathrm{B}}, b_{\mathrm{B}}\right)$ is illustrated in Fig. A.4. To systematically study the velocity width of disk and halo absorption along the antipodal sightline pairs we combined the COS data for individual antipodal pairs by multiplying the continuum-normalized spectra. In this way, we created composite velocity profiles for the strongest ion transition, Si III $\lambda 1206.50$, in the velocity range $v_{\mathrm{LSR}}=-500$ to $500 \mathrm{~km} \mathrm{~s}^{-1}$ (see Fig. 16, upper panel, for an example). For each of the 24 sightline pairs we define the total velocity width of the absorption in the composite spectrum as $\Delta v=v_{\max }-v_{\min }$. In the lower panel of Fig. 16 we show the velocity distribution of Si III $\lambda 1206.50$ absorption along these 24 antipodal composite profiles (with the contribution of the MS labeled in red). Twentythree out of the 24 antipodal profiles show halo absorption at either high negative or positive LSR velocities, and in 12 spectra absorption is seen at high positive and negative velocities. The plot illustrates the diversity of the absorption characteristics of the Milky Way disk and halo gas components, if seen from different vantage points.

This diversity is also seen in the upper panel of Fig. 17, where we show the distribution of $\Delta v$ in a histogram. $\Delta v$ ranges from 100 to $800 \mathrm{~km} \mathrm{~s}^{-1}$, while the distribution shows a strong peak at $\Delta v=300 \mathrm{~km} \mathrm{~s}^{-1}$. The antipodal sightline pairs with the largest values for $\Delta v$ are those passing the MS (see Fig. 16). This further highlights the importance of the MS for the overall 

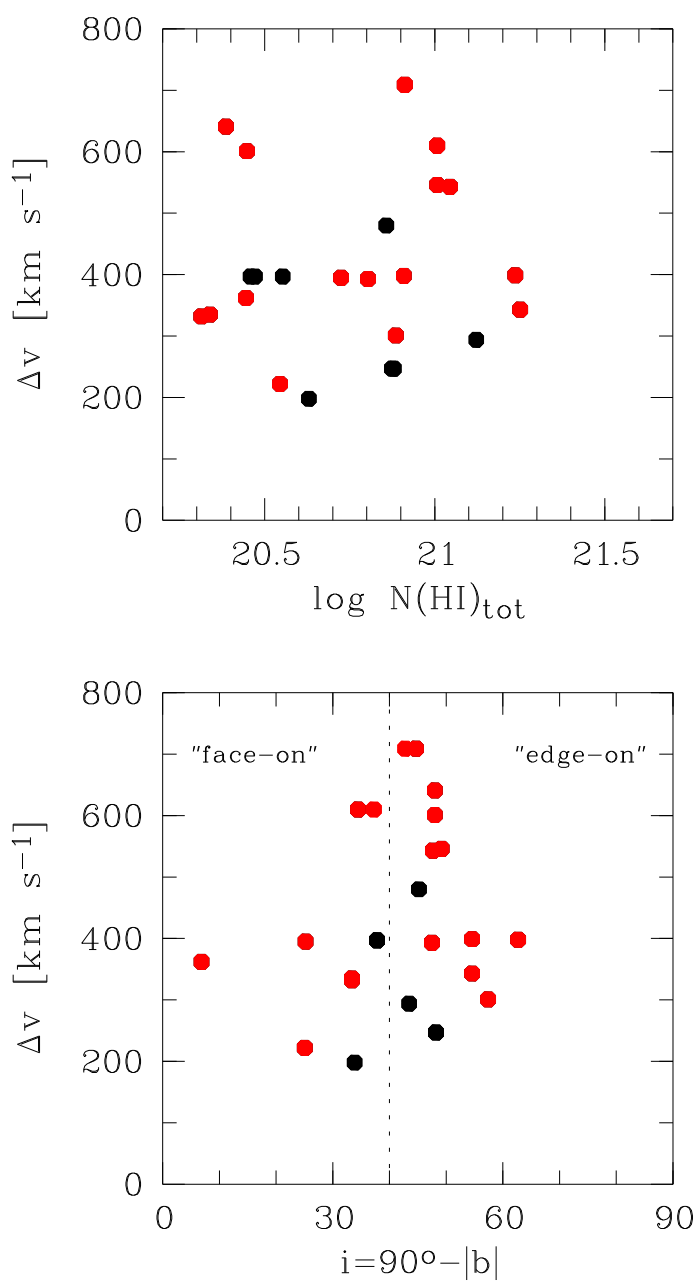

Fig. 18. Upper panel: total $\mathrm{H}$ I column densities are plotted against the velocity widths, $\Delta v$, for the 24 antipodal composite spectra. No systematic trend is visible. Lower panel: the velocity widths are plotted against the inclination $i$ of the Milky Way disk (assuming an external vantage point). The velocity widths are systematically higher for $i>40^{\circ}$ ("edgeon" case) than for $i \leq 40^{\circ}$ ("face-on" case). In both panels, MS sightlines are indicated in red.

absorption characteristics of the Milky Way as an absorption-line system.

\subsection{The Milky Way as a DLA}

We combined the kinematic information discussed in the previous section with information on the total H I column densities along the 24 antipodal sightlines to compare in detail the absorption characteristics of Milky Way disk and halo gas with the absorption properties of low-redshift QSO absorbers. In the lower panel of Fig. 17 we show the distribution of total (logarithmic) H I column densities along the 24 antipodal sightlines, as derived from integrating the combined $21 \mathrm{~cm}$ profiles. The total column densities vary considerably in a range between log $N(\mathrm{HI})=20.31$ and 21.25 with 71 percent of the sightlines having $\log N(\mathrm{HI}) \geq 20.5$. This distribution suggests that, from an external vantage point, $\log N(\mathrm{HI})$ (and thus the absorber type) would strongly depend on the viewing angle. The Milky Way disk and halo gas would be seen either as low-column density DLA $(\log N(\mathrm{HI})<20.5)$ or high-column density DLA $(\log N(\mathrm{HI}) \geq 20.5)$, if the sightline would pass the disk at the position of the Sun at an impact parameter of $b \approx 8 \mathrm{kpc}$.

The contribution of halo $\mathrm{H}$ I to the total H I column density is generally small. For 71 percent the column-density fraction of halo H I with $\left|v_{\mathrm{LSR}}\right| \geq 100 \mathrm{~km} \mathrm{~s}^{-1}$ with respect to the total neutral gas column along the antipodal sightline, $f_{\mathrm{HI}, \text { halo }}$, is less than 10 per cent. The median value of $f_{\mathrm{HI} \text {,halo }}$ is 0.055 . This implies that the total H I column density along the antipodal sightlines is determined predominantly by the intrinsic variations in the neutral gas columns in the Milky Way disk, if seen at different viewing angles. The total velocity spread of the absorption, in contrast, is governed predominantly by the large-scale distribution of Milky Way halo gas components along the different sightlines, which should be independent of the H I disk properties. In fact, we do not find any correlation between $\Delta v$ and $\log N(\mathrm{HI})_{\text {tot }}$ (Fig. 18, upper panel) in our sample.

Recent studies of strong metal absorbers and their host galaxies suggest that the velocity spread of the absorbing gas is related to the inclination $i$ of the galaxy and its absorbing ISM and CGM components with respect to the observer (Nielsen et al. 2015). The general trend in the Nielsen et al. sample is that $\Delta v$ has a maximum for blue (star-forming) "face-on" cases (with small values for $i$ ), possibly reflecting a kinematic imprint from outflows and winds along the projected minor axis.

To investigate the angular dependence of the absorption characteristics of Milky Way disk and halo gas along the different antipodal sightlines we transformed the absolute latitude $|b|$ into an inclination $i$ by using the relation $i=90^{\circ}-|b|$. In this way, sightlines passing through the Galactic poles are regarded as "faceon" sightlines, while those with small values of $|b|$ are considered as "edge-on" cases (see Nielsen et al. 2015, their Fig. 1). In Fig. 18, lower panel, we plot the velocity spread $\Delta v$ (from Si III $\lambda 1206.50)$ against $i$ for the 24 antipodal sightlines.

The velocity spread has its maximum in the range $i=40^{\circ}$ $50^{\circ}$, extending to very large values above $\Delta v \sim 700 \mathrm{~km} \mathrm{~s}^{-1}$, while for the "edge-on" case $\left(i>50^{\circ}\right)$ and the "face-on" case $(i<$ $\left.40^{\circ}\right)$ the values for $\Delta v$ are $<420 \mathrm{~km} \mathrm{~s}^{-1}$. The observed velocity spreads in the Milky Way thus are substantially (by a factor of a few) larger than those presented in Nielsen et al. (2015), who report values for $\Delta v$ of $<300 \mathrm{~km} \mathrm{~s}^{-1}$. It needs to be kept in mind that the DLAs in the Nielsen et al. sample spans a large range of impact parameters (with $b$ typically larger than $8 \mathrm{kpc}$ ). They also trace different types of DLA host galaxies that, in general, do not mimic the chemical and kinematic properties of nearby disk gas in the solar neighborhood. The primary origin for the discrepancy between the Milky Way $\Delta v$ range and that of DLAs is the MS, however. The MS alone spans a LSR velocity range of more than $800 \mathrm{~km} \mathrm{~s}^{-1}$ along the 24 antipodal sightlines (see Fig. 16).

In general, CGM features that arise from the interaction and merging of (satellite) galaxies presumably dominate the velocity spread of gas around Milky-Way type galaxies, as the velocity dispersion of satellite galaxies is expected to be higher than the gas infall- and outflow velocities. As a result, the kinematic signatures of outflows, winds, and accretion most likely are obscured by those of galaxy mergers. The cross section of merger-related gas streams around the local galaxy population is small, however (Gauthier et al. 2010; Martin et al. 2012), so that the observed large velocity dispersion of the Milky Way CGM is an exception, but not the rule. Possibly existing outflowing gas structures above the Milky Way disk, such as those belonging to the Fermi Bubbles (Fox et al. 2015), thus do not show up as separate features in the $i / \Delta v$ diagram. 


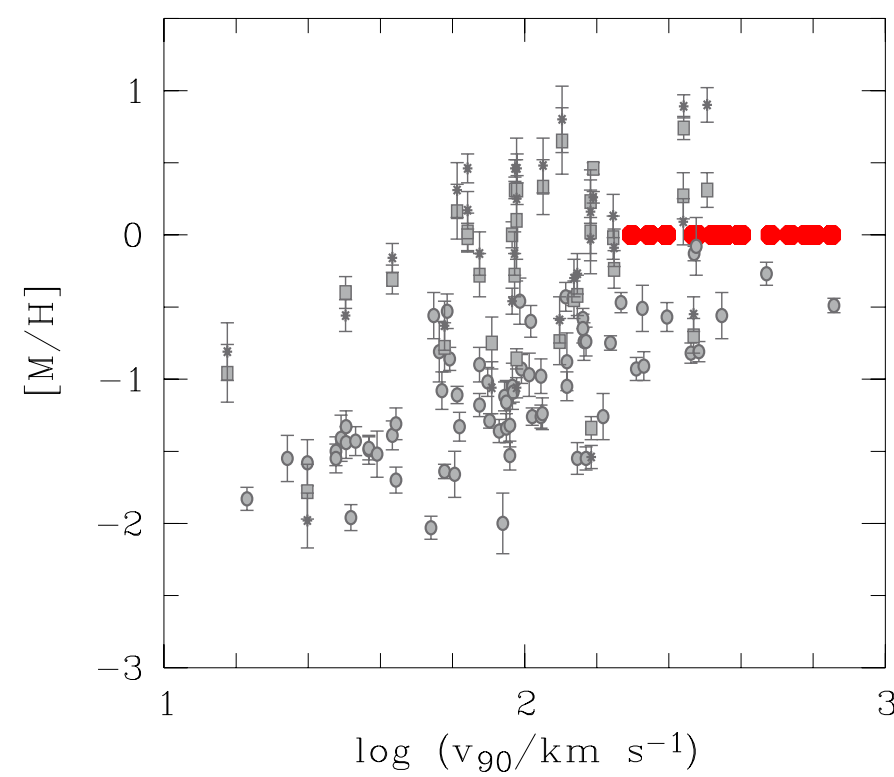

Fig. 19. The Milky Way's $\Delta v$ range (red filled circles) from the analysis (Fig. 16) of antipodal sightlines plotted on the velocity width/metallicity relation of DLAs (Som et al. 2015). The parameter $v_{90}$ denotes the velocity width in which 90 percent of the integrated optical depth is contained (Wolfe \& Prochaska 1998). We here set $v_{90} \equiv \Delta v$.

Another regularly used diagnostic tool to study strong absorption-line systems is the velocity width/metallicity relation for DLAs. In Fig. 19 we plot the $\Delta v$ range from the antipodal sightline analysis on the velocity width/metallicity relation presented by Som et al. (2015). For the Milky Way gas, we assume solar metallicities (i.e., $[\mathrm{M} / \mathrm{H}]=0$ ), which is justified because the mean metallicity should be a column-density weighted mean value, so that for the Milky Way (as DLA) it should be close to the value representative for the solar neighborhood. For the Milky Way antipodal sightlines the measured values for $\Delta v$ range from 100 to $800 \mathrm{~km} \mathrm{~s}^{-1}$ (see Fig. 17), thus being broadly consistent with values of $\Delta v$ measured for high-metallicity DLAs. The majority of the MW data points lie, however, at the high end of the $\Delta v$ distribution for $[\mathrm{M} / \mathrm{H}]=0$ absorbers, which we again attribute to the fact that large values for $\Delta v$ in the MW are caused by gas from the MS, i.e., from merger processes, which are believed to be rare in DLA samples. If such a large scatter in $\Delta v$ was typical for low-redshift $L^{\star}$ galaxies, the individual data points on DLA scaling relations must be interpreted with great caution, as the observed parameters (H I column density, velocity spread) may not reflect at all representative properties of the absorber host galaxy.

\subsection{Comparison with Local Group simulations}

Cosmological hydrodynamical simulations predict that group environments within the overall cosmic web are filled with large amounts of diffuse gas whose mass exceeds that of gas inside the galaxies (e.g., Raouf et al. 2016). In their recent study, N14 have explored the large-scale distribution and overall physical properties of gas in the Local Group based on simulation data from the Constrained Local UniversE Simulations (CLUES) project (www.clues-project.org), which aims at simulating a realistic LG environment including local features and the most prominent surrounding cosmological structures. To evaluate the large-scale properties of LG gas and its possible signatures in
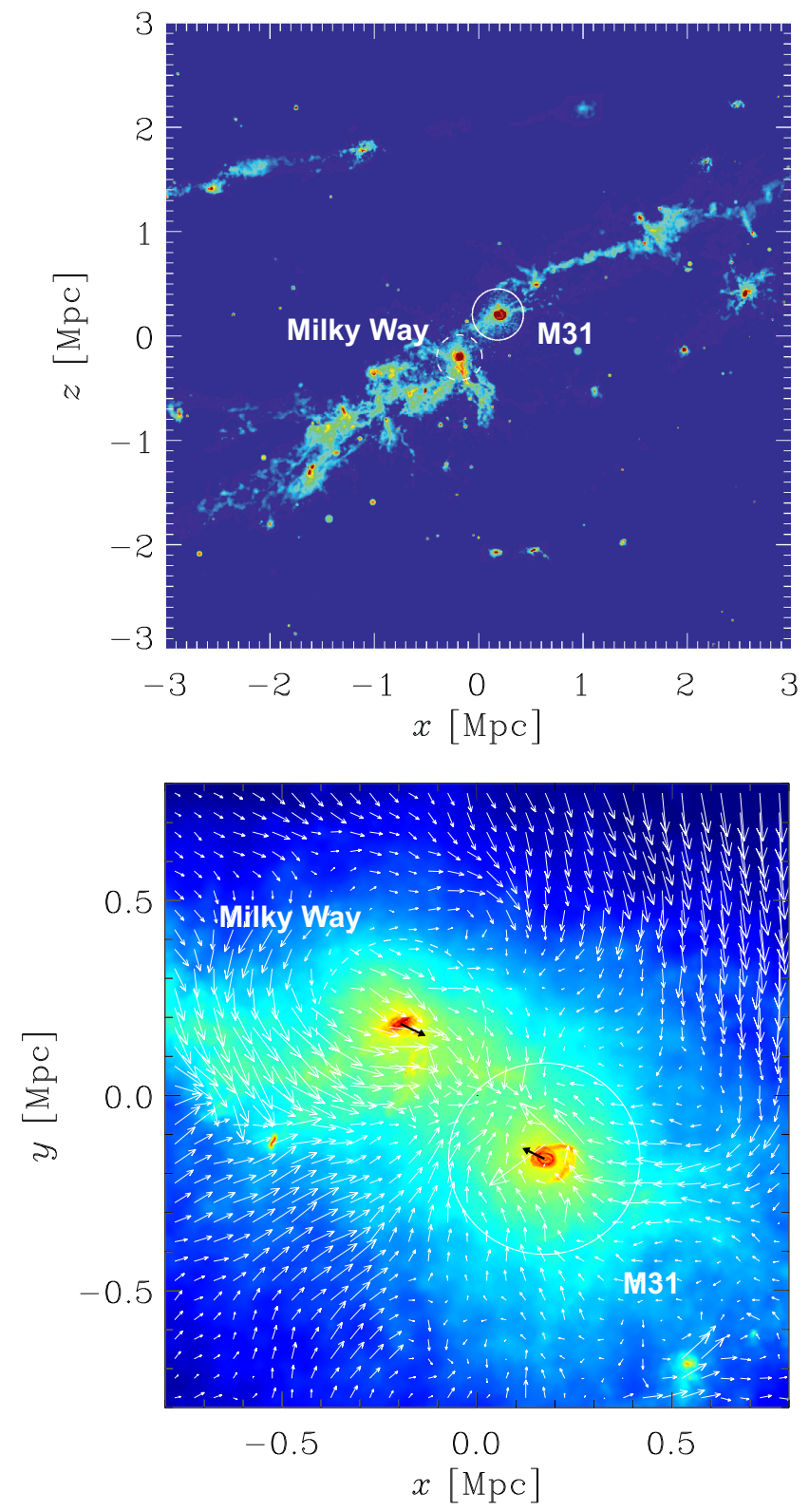

Fig. 20. Upper panel: spatial distribution of neutral hydrogen gas at $T<10^{5} \mathrm{~K}$ in the large-scale environment of the Local Group, as predicted from a constrained cosmological simulation from the CLUES project (N14). The color scale reflects the total hydrogen gas density, where the green filamentary structures outside the virial radii of the LG member galaxies lie in the density range $n_{\mathrm{H}}=10^{-5}-10^{-3} \mathrm{~cm}^{-3}$. Positions and virial radii of Milky Way and M31 are indicated with white dashed (Milky Way) and solid (M31) circles. Lower panel: zoom-in of the gas distribution in and around the simulated MW/M31 galaxies and the bulk motions of that gas with respect to the Local Group barycenter in the $x / y$ plane. The white arrows show the gas velocity field, with the largest ones representing a velocity of $130 \mathrm{~km} \mathrm{~s}^{-1}$. The black arrows indicate the velocities of the MW and M31 galaxies. Their absolute space velocities are 67 and $76 \mathrm{~km} \mathrm{~s}^{-1}$, respectively. The bright colors again indicate regions of enhanced hydrogen density.

FUV absorption-line data we here reanalyze the simulations of N14.

We focus particularly on a side-aspect of the N14 study, namely the spatial distribution of LG gas outside the virial radius of the Milky Way. We also want to explore the kinematics of the LG gas with respect to the space motion of the MW disk 
and its rotation and compare it with the velocity distribution of the HVCs seen in our COS data.

The initial matter distribution in the CLUES simulations consists of a cubic box of $64 h^{-1} \mathrm{Mpc}$ side length with a highresolution spherical region of $2 h^{-1} \mathrm{Mpc}$ radius located at its center. The masses of the gas and dark matter particles inside the high-resolution region are $M_{\text {gas }}=3.89 \times 10^{5} h^{-1} M_{\odot}$ and $M_{\mathrm{DM}}=1.97 \times 10^{6} h^{-1} M_{\odot}$, respectively. Further details on the setup and realization of the CLUES simulations as well as a detailed description of the overall results from that simulation can be found in N14 and references therein. Note that the following results are specific to the CLUES simulations and their cosmological representation of the LG. It would need to be tested whether they could be reproduced using different simulations with different initial conditions and physical recipes.

In the upper panel of Fig. 20 we show the spatial distribution of neutral hydrogen ( $\mathrm{H}$ I at $T<10^{5} \mathrm{~K}$ ) in the large-scale environment of the LG at $z=0$. The gas distribution is relatively patchy and forms a bar-like structure that follows closely the elongated shape of the cosmological filament that hosts the LG. Milky Way and M31 (white circles denoting their virial radii) reside in the center of this structure. Obviously, there are substantial amounts of diffuse warm gas outside the virial radii of MW and M31 that potentially could give rise to UV absorption if seen from the MW disk.

To display the large-scale kinematics of the gas around MW and M31 we show in the lower panel of Fig. 20 the velocity vectors (with respect to the LG barycenter) of the simulated gas particles (white arrows) and the two galaxies (black arrows) in the $x / y$ plane. The galaxies move towards the LG barycenter while the ambient gas is circulating around MW and M31 in a complex pattern of infall- and outflow channels. Because of the complex gas motions, the velocity components in the direction of the LG barycenter are smaller for the gas particles than for the two galaxies. As a result, there is a systematic net-motion of both galaxies towards the LG barycenter with respect to the ambient LG gas: MW and M31 are ramming into LG gas that lies in-between them near the LG barycenter, while they are moving away from gas that lies behind them in the opposite direction in the outer regions of the LG filament.

To predict the velocities of UV absorption based on this simulation we take into account the rotational motion of the MW disk. We assume that the simulated MW disk has a circular orbit speed at the position of the Sun of $v_{\text {rot }}=255 \mathrm{~km} \mathrm{~s}^{-1}$, as recently derived by Reid et al. (2014). Taking all this spatial and kinematic information into account we prepared maps for the sky distribution of the LG gas from the simulation in a similar way as we prepared the maps for the observational data. In the upper panel of Fig. 21 we show the density map of LG gas outside the MW virial radius. The bar-like gaseous LG filament (see Fig. 20, upper panel) reaches from the general M31 direction (in the simulation: $\left.(l, b)=\left(60^{\circ},-55^{\circ}\right)\right)$ roughly to its antipole on the sky (see also N14)). In the lower panel, we plot the expected radial LSR velocity of the LG gas as a function of $(l, b)$ including the effect of Galactic rotation. A dipole pattern is clearly visible. Only at low latitudes $\left(|b|<30^{\circ}\right)$ is Galactic rotation relevant for $\left|v_{\mathrm{LSR}}\right| \geq 200 \mathrm{~km} \mathrm{~s}^{-1}$. The observed velocity dipole for gas at higher (absolute) latitudes and velocities instead is a result of the relative motion of the Milky Way with respect to the surrounding gas in the direction of the LG barycenter. From the combination of the velocity and density information in the two panels in Fig. 21 follows that LG gas at $|b|>30^{\circ}$, if it were cold and dense enough to be observed in UV absorption, would imprint a dipole absorption pattern on the sky, with redshifted high-velocity absorption $\left(v_{\mathrm{LSR}} \geq 200 \mathrm{~km} \mathrm{~s}^{-1}\right)$ at $l>200^{\circ}, b>0^{\circ}$ and blue-shifted high-velocity absorption $\left(v_{\mathrm{LSR}} \leq-200 \mathrm{~km} \mathrm{~s}^{-1}\right)$ at $l<120^{\circ}, b<0^{\circ}$.

As discussed earlier, such a dipole is indeed seen in our COS survey (NIR, SIR; Sect. 5.4; Figs. 2, 8, 11). It also has been recognized in our previous O VI survey (Sembach et al. 2003; Wakker et al. 2003) and other studies of intermediate and high ions (e.g., Collins et al. 2005). A definite answer, whether the observed dipole-absorption features are indeed related to LG gas yet cannot be given here, because the MS similarly imprints an emission/absorption dipole pattern on the sky, as evident from Fig. 8. Since a reliable distance determination of this gas appears to be out of reach, a precise measurement of its chemical composition (in comparison with abundances found in the MS) might shed light on its origin.

In summary, the CLUES simulations support (but do not require) the idea that some of the observed absorbers in the NIR and SIR represent ionized LG gas beyond the virial radius of the Milky Way that follows the large-scale flow of matter in the LG.

\section{Conclusions}

In this paper, we present the largest available survey of UV absorption in Galactic HVCs in the velocity range $\left|v_{\mathrm{LSR}}\right|=100$ $500 \mathrm{~km} \mathrm{~s}^{-1}$, tracing the extended gaseous halo and CGM of the Milky Way and gas in the Local Group. Our survey is based on the analysis of various lines from the metal ions Si II, Si III, C II, and C IV in 270 spectra of extragalactic UV background sources observed with HST/COS. We supplement our COS spectra with H I $21 \mathrm{~cm}$ data from GASS and EBHIS, as well as with data from constrained cosmological simulations from the CLUES project. We update previous results from smaller HVC surveys (e.g., Lehner et al. 2012; Herenz et al. 2013) concerning the HVC's sky distribution, their internal structure, their ionization properties, and estimate the total HVC mass and accretion rate. Based on our excellent database, we further present several new results on HVC aspects that have not yet been studied before. We analyze the angular two-point correlation function of HVC absorption and analyze small-scale variations in the UV absorption patterns. We further investigate the relation between high-velocity UV absorption and gas in other LG galaxies and the LG's intragroup medium. We systematically compare the UV absorption characteristics of Milky Way disk and halo gas along antipodal sightlines with those of low-redshift DLAs. Finally, we discuss the role of HVCs in a cosmological context by comparing the observed gas properties with those seen in constrained simulations of the LG.

We summarize the main results of our study as follows:

(1) Sky-covering fraction of HVCs. The overall all-sky covering fraction of high-velocity absorption in our survey is $f_{\mathrm{c}}=77 \pm 6$ percent, as derived from the analysis of the most sensitive ion, $\mathrm{Si}$ III for $N_{\text {lim }}(\mathrm{Si}$ III $)=12.1$. For the other three ions we obtain covering fractions of $f_{\mathrm{c}}(\mathrm{Si}$ II $)=69 \pm 6$ percent for $N_{\text {lim }}(\mathrm{Si}$ II $)=12.3, f_{\mathrm{c}}(\mathrm{C}$ II $)=70 \pm 6$ percent for $N_{\text {lim }}(\mathrm{C}$ II $)=13.2$, and $f_{\mathrm{c}}(\mathrm{C} \mathrm{IV})=58 \pm 7$ percent for $N_{\lim }(\mathrm{C}$ IV $)=12.9$. The $\mathrm{Si}$ III detection rate is $\sim 4-5$ times higher than that for $\mathrm{HI}$ for $\log N(\mathrm{HI}) \geq 18.7$ along the same sightlines, as estimated from the complementary 21 $\mathrm{cm}$ data from GASS and EBHIS. Most of the detected highvelocity absorbers can be kinematically attributed to known H I $21 \mathrm{~cm}$ gas complexes, such as the MS, Complex C, Complex A, and others. This implies that the large cross section of high-velocity UV absorption is mostly due to the ionized 


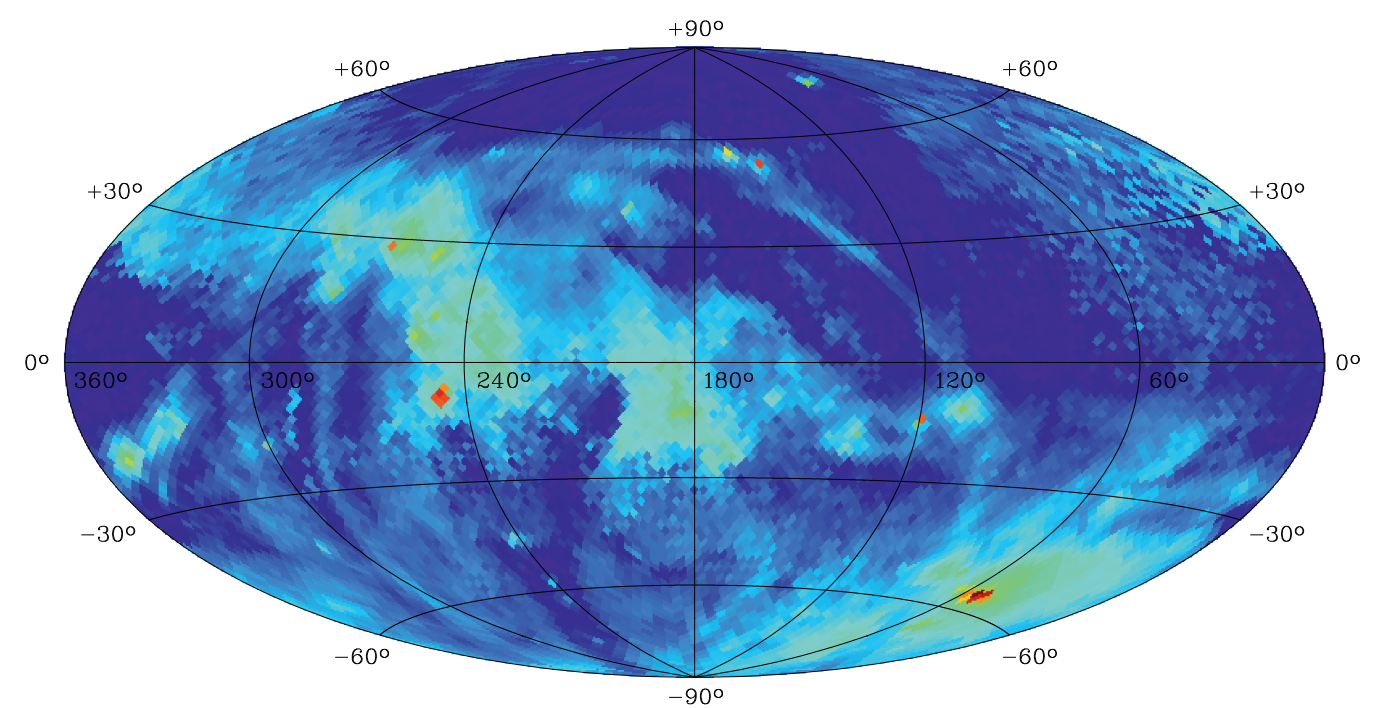

$-6.8$

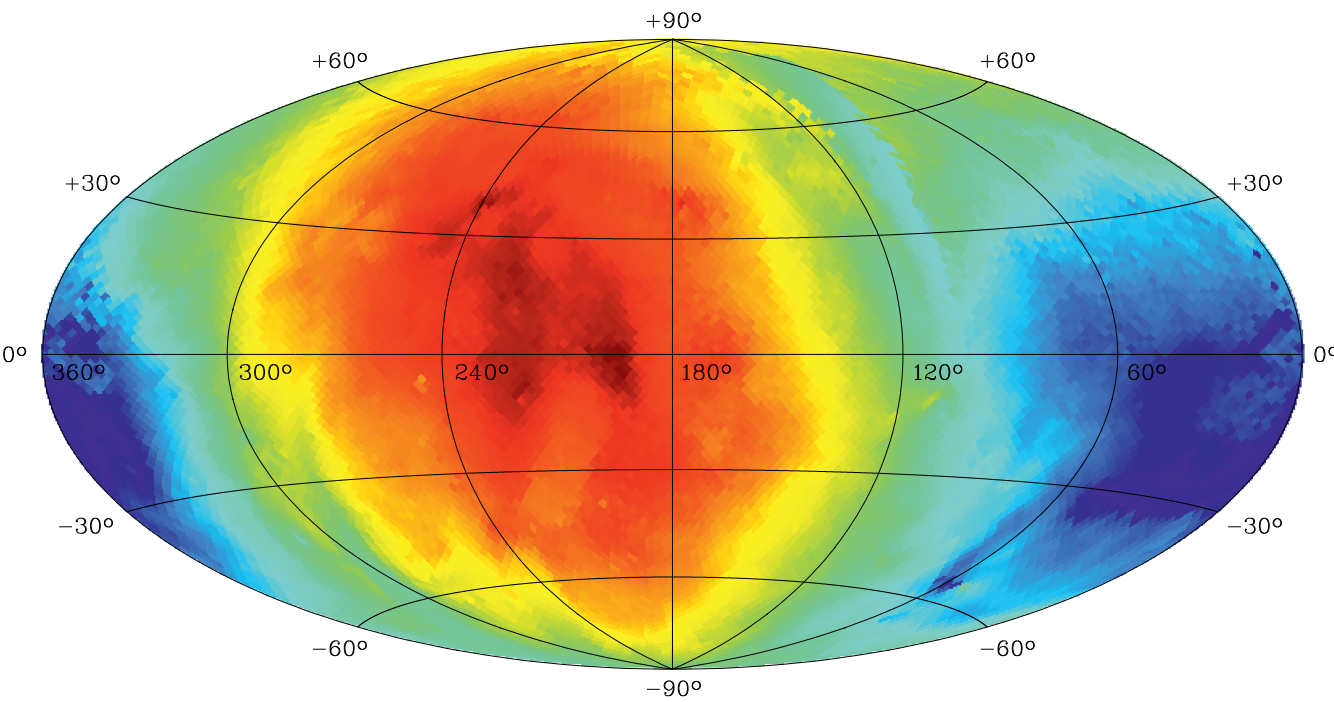

$-450$

LSR Velocity $\left[\mathrm{km} \mathrm{s}^{-1}\right]$

$+300$

Fig. 21. Upper panel: Hammer-Aitoff projection of the gas density in the Local Group (for gas beyond the virial radius of the Milky Way), as seen in the CLUES simulation from the position of the Sun. M31 appears as a red/yellow spot near $(l, b)=\left(60^{\circ},-55^{\circ}\right)$. The gaseous LG filament is indicated with the greenish color, which displays gas at densities in the range $\log n_{\mathrm{H}}=-4$ to -3 . The filament reaches from the general M31 direction roughly to its antipole on the sky. Two smaller dwarf galaxies are visible as red spots. Lower panel: velocity distribution of the gas in the same projection, given in LSR. The observed dipole pattern separates gas with high positive velocities at $l=120^{\circ}-320^{\circ}$ from gas at high negative velocities $\left(l<120^{\circ}, l>320^{\circ}\right)$.

envelopes of the neutral HVC cores. The overall HVC absorption fraction is systematically lower in the northern sky than in the southern hemisphere (e.g., $f_{\mathrm{c}}(\mathrm{Si}$ III $)=73 \pm 7$ in the north vs. $f_{\mathrm{c}}(\mathrm{Si}$ III $)=89 \pm 13$ in the south). We attribute this asymmetry to the widespread presence of diffuse (predominantly ionized) gas from the MS at negative radial velocities that covers a significant fraction of the sky at $b<0^{\circ}$. We identify two antipodal regions at $l>240^{\circ}, b>60^{\circ}$ in the northern sky (northern ionized region, NIR) and at $l<120^{\circ}, b=-60^{\circ}-0^{\circ}$ in the south (southern ionized region, SIR) that show prominent UV absorption at very high (absolute) radial velocities $\left(\left|v_{\mathrm{LSR}}\right| \geq 200 \mathrm{~km} \mathrm{~s}^{-1}\right.$ ), but with only very little H I $21 \mathrm{~cm}$ emission.

(2) The multi-phase nature of HVCs. The simultaneous detection of Si II, Si III, C II, C IV, and H I in many high-velocity absorption components implies the presence of multiple gas phases (from neutral to highly ionized gas) in the CGM along each sightline. The substantially higher covering fraction of high-velocity UV absorption compared to high-velocity H I $21 \mathrm{~cm}$ emission demonstrates that hydrogen is predominantly ionized in these structures. Many regions that exhibit high-velocity UV absorption without corresponding $21 \mathrm{~cm}$ 
emission represent the ionized outer layers of nearby neutral HVC complexes, as indicated by the same large-scale kinematics. From the observed spatial and kinematic variations of the column-density ratios $\mathrm{Si}$ II/Si III, C II/Si III, and $\mathrm{C}$ IV/Si III follows that there is substantially more ionized gas at high negative radial velocities than at high positive velocities. Again, this trend is related to diffuse gas associated with the MS. Particularly high C IV/Si III are found in the NIR and SIR, indicating that these regions contain mostly diffuse, ionized gas.

(3) Spatial clustering of HVCs and their internal structure. We analyze the angular distribution of high-velocity absorption by constructing the two-point correlation function, $\xi$, for HVC components in our QSO sample. We find that $\xi(\theta)$ exhibits a strong clustering signal for angular separations of $\theta \leq 30^{\circ}$, while $\xi$ drops quickly for larger angles. From the analysis of 12 sightline pairs with small angular separations (down to $\theta \sim 0.5^{\circ}$ ) we find significant variations in the equivalent widths of high-velocity features, while the overall velocity structure in the HVC absorption patterns remains mostly unchanged at the spectral resolution of COS. For typical HVC distances in the range $d=5-55 \mathrm{kpc}$ these findings indicate that high-velocity absorbers in the Milky Way halo represent coherent gas structures on scales of a few up to a few hundred kpc that follow a large-scale kinematics and that exhibit internal variations in gas density/physical conditions on pc scales.

(4) Relation between HVCs and other LG galaxies. We systematically investigate sightlines that pass the outskirts of several Local Group galaxies outside the virial radius of the MW to search for absorption signatures that might be related to the CGM of these galaxies. Along seven sightlines, highvelocity absorption from extended halo gas around M31 most likely is present in the LSR velocity range between -300 and $-150 \mathrm{~km} \mathrm{~s}^{-1}$, in line with the findings recently presented by Lehner et al. (2015). No CGM absorption is found at impact parameters $\rho \leq 2 R_{\mathrm{vir}}$ and velocities close to the systemic galaxy velocities for NGC 3109, Antila, Sextans A, Aquarius (one sightline per galaxy, respectively), and Leo I (12 sightlines). Absorption is found in a sightline passing IC 1613 at $\rho \sim 1.5 R_{\text {vir }}$ near the systemic velocity of IC 1613 , but this absorption most likely is associated with gas from the MS. Therefore, only for M31 and the Milky Way the COS data provides compelling evidence for the presence of an extended CGM, while for the LG dwarfs/satellites there are no compelling hints for a circumgalactic gas component.

(5) Local Group gas. We further investigate the distinct gas properties and kinematics of the NIR and SIR (see definition above) by relating the observed HVC absorption characteristics in these regions with the kinematics of LG galaxies and with predictions from hydrodynamical simulations of LG gas. The NIR and SIR, for which no distance information is available, form a dipole on the sky with opposite signs for the observed absorption velocities. Because of the observed high radial velocities and the high absolute Galactic latitudes of these antipodal regions, the observed dipole pattern cannot be produced by the rotation of the Galactic disk. We show that the galaxies outside the virial radius of the Milky Way form a similar dipole-velocity pattern on the sky, which reflects the general flow of galaxies in the LG towards the LG barycenter, the Milky Way being part of that flow. Using constrained cosmological simulations of the LG and its gaseous environment from the CLUES project (N14) we further demonstrate that LG gas, that circulates around
LG member galaxies within the overall matter flow, is indeed expected to form a dipole-velocity pattern that matches well the observed one for the NIR/SIR. This, together with the well-established presence of high-velocity O VI in these directions at similar high radial velocities (Sembach et al. 2003; Wakker et al. 2003), leads us to speculate that the NIR and the SIR traces LG gas that follows the large-scale kinematics of matter in the cosmological filament that forms the LG.

(6) Total mass and infall rate of HVCs. From the observed ion covering fractions, previously measured HVC distances and metallicities as well as the measured total $\mathrm{Si}$ and $\mathrm{C}$ column densities in HVCs we estimate a total gas mass of the Milky Way's HVCs of $M_{\mathrm{HVC}} \geq 3.0 \times 10^{9} M_{\odot}$. This is $\geq 43$ percent of the ISM gas mass in the Galactic disk. We find that the total gas accretion rate in the form of neutral and ionized HVCs is $\geq 6.1 M_{\odot} \mathrm{yr}^{-1}$. The MS, here assumed to be located at a distance of $d=55 \mathrm{kpc}$, dominates the total HVC mass and gas-accretion rate by far, while all other HVCs contribute with only $\leq 10$ percent. If much of the MS would be located at distances $d=100-150 \mathrm{kpc}$ the gas accretion rate would be even higher by a factor of $\sim 2-3$. The infall of HVCs from the halo to the disk thus represents a significant mass inflow that may substantially influence the future star-formation rate in the Milky Way.

(7) The Milky Way as a DLA. From our data set we identify 24 sightline-pairs with antipodal directions within $6^{\circ}$. We create composite spectra for these antipodal sightlines to study the integrated absorption characteristics of the Milky Way disk and halo gas from different vantage points and compare the results with recent studies of QSO absorption-line systems. The total column densities along the 24 antipodal sightlines show a substantial scatter, ranging from log $N(\mathrm{HI})=20.31$ to 21.25 , with 71 percent of the sightlines having $\log N(\mathrm{HI}) \geq 20.5$. Thus, the Milky Way disk and halo gas (at the position of the Sun) would be seen as a DLA from an external vantage point, with a total gas column that depends (non-systematically) on the viewing angle. We find that also the total velocity width, $\Delta v$, of Si III absorption in the various disk and halo absorption components shows a huge spread along the 24 antipodal directions. $\Delta v$ varies from 100 to $800 \mathrm{~km} \mathrm{~s}^{-1}$ and the $\Delta v$ distribution shows a peak at $300 \mathrm{~km} \mathrm{~s}^{-1}$. With such large velocity spreads, the Milky Way disk/halo gas lies at the extreme end of the $\Delta v$ distribution of low- $z$ DLAs, but still fits to the general velocity-width vs. metallicity relation for DLAs and sub-DLAs (Som et al. 2015). The dominant contribution to the large velocity spread of Milky Way gas comes from the MS, again indicating the dominant role of the Stream for the Milky Way's CGM absorption properties. Since the cosmological cross section of merger-related gas streams around galaxies is small, the MW thus would represent an a-typical DLA if seen from far away.

Acknowledgements. This research used the facilities of the Canadian Astronomy Data Centre operated by the National Research Council of Canada with the support of the Canadian Space Agency. S. E. N. acknowledges support from the Deutsche Forschungsgemeinschaft (DFG) under the grant NU 332/2-1. Support was provided by NASA through grants HST-GO-12604.01-A (Wakker), HSTGO-13448.01-A (Wakker), HST-GO-13840.05-A (Wakker), HST-GO-12982 (Lehner), HST-AR-12854 (Lehner), and HST-AR-12846.001 (Charlton) from the Space Telescope Science Institute, which is operated by the Association of Universities for Research in Astronomy, Incorporated, under NASA contract NAS5-26555. We thank an anonymous referee for helpful comments and suggestions. 


\section{References}

Asplund, M., Grevesse, N., Sauval, A. J., \& Scott, P. 2009, ARA\&A, 47, 481 Barger, K. A., Lehnerm, N., \& Howk, J. C. 2016, ApJ, 817, 91

Ben Bekhti, N., Richter, P., Westmeier, T., \& Murphy, M. T. 2008, A\&A, 487, 583

Ben Bekhti, N., Winkel, B., Richter, P., et al. 2012, A\&A, 542, A110

Besla, G., Kallivayalil, N., Hernquist, L., et al. 2012, MNRAS, 421, 210

Bland-Hawthorn, J., Maloney, P., Sutherland, R. S., \& Madsen, G. J. 2013 ApJ,778, 58

Blitz, L., Spergel, D. N., Teuben, P. J., Hartmann, D., \& Burton, W. B. 1999, ApJ, 514, 818

Bluhm, H., de Boer, K. S., Marggraf, O., \& Richter, P. 2001, A\&A, 367, 299

Bosler, T. L., Smecker-Hane, T. A., \& Stetson, P. B. 2007, MNRAS, 378, 318

Bowen, D. V., Tolstoy, E., Ferrara, A., Blades, J. C., \& Brinks, E. 1997, ApJ, 478,530

Chomiuk, L., \& Povich, M. S. 2011, AJ, 142, 197

Collins, J. A., Shull, J. M., \& Giroux, M. L. 2005, ApJ, 623, 196

Collins, J. A., Shull, J. M., \& Giroux, M. L. 2009, ApJ, 705, 962

Danforth, C. W., Howk, J. C., Fullerton, A. W., Blair, W. P., \& Sembach, K. R. 2002, ApJS, 139, 81

Davis, M., \& Peebles, P. J. E. 1983, ApJ, 267, 465

de Avillez, M. A., \& Breitschwerdt, D. 2007, ApJ, 665, L35

de Boer, K. S., \& Savage, B. D. 1980, ApJ, 238, 86

Debes, J., et al. 2016, Cosmic Origins Spectrograph Instrument Handbook, Version 8.0 (Baltimore, MD: STScI)

Dehnen, W., McLaughlin, D. E., \& Sachania, J. 2006, MNRAS, 369, 1688

Dickey, J. M., \& Lockman, F. J. 1990, ARA\&A, 28, 215

Dieter, N. H. 1971, A\&A, 12, 59

D’Onghia, E., \& Fox, A. J. 2016, ARA\&A, 54, 363

Ferriere, K. 2001, Rev. Mod. Phys., 73, 1031

Fox, A. J., Wakker, B. P., Savage, B. D., et al. 2005, ApJ, 630, 332

Fox, A. J., Savage, B. D., \& Wakker, B. P. 2006, ApJS, 165, 229

Fox, A. J., Wakker, B. P., Smoker, J. V., et al. 2010, ApJ, 718, 1046

Fox, A. J., Richter, P., Wakker, B. P., et al. 2013, ApJ, 772, 110

Fox, A. J., Wakker, B. P., Barger, A., et al. 2014, ApJ, 787, 147

Fox, A. J., Bordoloi, R., Savage, B. D., et al. 2015, ApJ, 799, L7

Gallart, C., Freedman, W. L., Aparicio, A., Bertelli, G., \& Chiosi, C. 1999, AJ, 118,2245

Gauthier, J.-R., Chen, H.-W., \& Tinker, J. L. 2010, ApJ, 716, 1263

Green, J. C., Froning, C. S., Osterman, S., et al. 2012, ApJ, 744, 60

Gupta, A., Mathur, S., Krongold, Y., Nicastro, F., \& Galeazzi, M. 2012, ApJ, 756, L8

Herenz, P., Richter, P., Charlton, J. C., \& Masiero, J. R. 2013, A\&A, 550, A87

Heitsch, F., \& Putman, M. E. 2009, ApJ, 698, 1485

Jin, S., \& Lynden-Bell, D. 2008, MNRAS, 383, 1686

Joung, M. R., Putman, M. E., Bryan, G. L., Fernandez, X., \& Peek, J. E. G. 2012 , ApJ, 759, 137

Kalberla, P. M. W., Burton, W. B., Hartmann, D., et al. 2005, A\&A, 440, 775

Kalberla, P. M. W., McClure-Griffiths, N. M., Pisano, D. J., et al. 2010, A\&A, 521,17

Keeney, B. A., Danforth, C. W., Stocke, J. T., et al. 2006, ApJ, 646, 951

Kerp, J., Winkel, B., Ben Bekhti, N., Floer, L., \& Kalberla, P. M. W. 2011, Astron. Nachr., 332, 637

Lehner, N., \& Howk, J. C. 2007, MNRAS, 377, 687

Lehner, N., \& Howk, J. C. 2010, ApJ, 709, 138

Lehner, N., \& Howk, J. C. 2011, Sci, 334, 955

Lehner, N., Staveley-Smith, L., \& Howk, J. C. 2009, ApJ, 702, 940

Lehner, N., Howk, J. C., Thom, C., et al. 2012, MNRAS, 424, 2896

Lehner, N., Howk, J. C., \& Wakker, B. P. 2015, ApJ, 804, 79

Liang, C. J., \& Chen, H.-W. 2014, MNRAS, 445, 2061

Lockman, F. J., Murphy, E. M., Petty-Powell, S., \& Urick, V. J. 2002, ApJS, 140 331

Marggraf, O., \& de Boer, K. S. 2000, A\&A, 363, 733

Martin, C. L., Shapley, A. E., \& Coil, A. 2012, ApJ, 760, 127

Mathewson, D. S., Cleary, M. N., \& Murray, J. D. 1974, ApJ, 190, 291

McClure-Griffiths, N. M., Pisano, D. J., Calabretta, M. R., et al. 2009, ApJS 181,398

McMillan, P. J. 2011, MNRAS, 414, 2446

Miller, M. J., \& Bregman, J. N. 2015, ApJ, 800, 14

Miller, M. J., Kluck, E. J., \& Bregman, J. N. 2016, ApJ, 818, 112

Morton, D. C. 2003, ApJS, 149, 205

Murray, C. E., Stanimirovicz, S., McClure-Griffithsm N. M., et al. 2015, ApJ, 808,41
Nicastro, F., Zezas, A., Elvis, M., et al. 2003, Nature, 421, 719

Nielsen, N. M., Churchill, C. W., Charlron, J. C., et al. 2015, ApJ, 811, 132

Nuza, S. E., Parisi, F., \& Scannapieco, C., et al. 2014, MNRAS, 441, 2593

Peebles, P. J. E., Phelps, S. D., Shaya, E. J., \& Tully, R. B. 2001, ApJ, 554, 104

Peebles, P. J. E., Tully, R. B., \& Shaya, E. J. 2011, unpublished [arXiv: 1105.5596]

Prochaska, J. X. 2017, Astronomy \& Computing, 19, 27

Putman, M. E., Staveley-Smith, L., Freeman, K. C., Gibson, B. K., \& Barnes, D. G. 2003, ApJ, 586, 170

Putman, M. E., Peek, J. E. G., \& Joung, M. R. 2012, ARA\&A, 50, 491

Rao, S. M., Sardane, G., \& Turnshek, D. A. 2013, MNRAS, 432, 866

Raouf, M., Khosroshabi, H. G., \& Dariush A. A. 2016, ApJ, 824, 140

Reid, M. J., Menten, K. M., Brunthaler, A., et al. 2014, ApJ, 783, 130

Richter, P. 2006, Rev. Mod. Astron., 19, 31

Richter, P. 2012, ApJ, 750, 165

Richter, P. 2017, in Gas Accretion onto Galaxies, Astrophysics and Space Science Library, eds. A. J. Fox, \& R. Davé (Springer), 15

Richter, P., de Boer, K. S., Bomans, D. J., et al. 1999, Nature, 402, 386

Richter, P., Sembach, K. R., Wakker, B. P., \& Savage, B. D. 2001a, ApJ, 562, L181

Richter, P., Savage, B. D., Wakker, B. P., Sembach, K. R., \& Kalberla, P. M. W. 2001b, ApJ, 549, 281

Richter, P., Sembach, K. R., Wakker, B. P., et al. 2001c, ApJ, 559, 318

Richter, P., Charlton, J. C., Fangano, A. P. M., Ben Bekhti, N., \& Masiero, J. R. 2009, ApJ, 695, 1631

Richter, P., Krause, F., Fechner, C., Charlton, J. C., \& Murphy, M. T. 2011, A\&A, 528, A12

Richter, P., Fox, A. J., Wakker, B. P., et al. 2013, ApJ, 772, 111

Richter, P., Fox., A. J., Ben Bekhti, N., et al. 2014, Astron. Nachr., 335, 92

Richter, P., Wakker, B. P., Fechner, C., et al. 2016, A\&A, 590, A68

Robitaille, T. P., \& Whitney, B. A. 2010, ApJ, 710, L11

Ryans, R. S. I., Keenan, F. P., Sembach, K. R., \& Davies, R. D. 1997, MNRAS, 289,986

Savage, B. D., \& Sembach, K. R. 1991, ApJ, 379, 245

Sembach, K. R., Howk, J. C., Savage, B. D., \& Shull, J. M. 2001, AJ, 121, 992

Sembach, K. R., Wakker, B. P., Savage, B. D., et al. 2003, ApJS, 146, 165

Sembach, K. R., Wakker, B. P., \& Tripp, T. M. 2004, ApJS, 150, 387

Shull, J. M., Jones, J. R., Danforth, C. W., \& Collins, J. A. 2009, ApJ, 699, 754

Smoker, J. V., Fox, A. J., \& Keenan, F. P. 2015, MNRAS, 451, 4346

Som, D., Kulkarni, V. P., Meiring, J., et al. 2015, ApJ, 806, 25

Stocke, J. T., Keeney, B. A., Danforth, C. W., et al. 2013, ApJ, 763, 148

Stocke, J. T., Keeney, B. A., Danforth, C. W., et al. 2014, ApJ, 791, 128

Thom, C., Putman, M. E., Gibson, B. K., et al. 2006, ApJ, 638, L97

Thom, C., Peek, J. E. G., Putman, M. E., et al. 2008, ApJ, 684, 364

Trenti, M., Stiavelli, M., Bouwens, J. R., et al. 2010, ApJ, 714, L202

Tripp, T. M., Wakker, B. P., Jenkins, E. B., et al. 2003, AJ, 125, 3122

Tumlinson, J., Thom, C., Werk, J. K., et al. 2013, ApJ, 777, 59

Wakker, B. P. 2001, ApJS, 136, 463

Wakker, B. P. 2004, in High Velocity Clouds, eds. H. van Woerden, B. P. Wakker,

U. J. Schwarz, \& K. S. de Boer (Dordrecht: Kluwer), 25

Wakker, B. P. 2006, ApJS, 163, 282

Wakker, B. P., \& van Woerden, H. 1997, ARA\&A, 35, 217

Wakker, B. P., Howk, J. C., \& Savage, B. D. 1999, Nature, 402, 388

Wakker, B. P., Ooserloo, T., \& Putman, M. E. 2002, AJ, 123, 1953

Wakker, B. P., Savage, B. D., Sembach, K. R., et al. 2003, ApJS, 146, 1

Wakker, B. P., York, D. G., Howk, J. C., et al. 2007, ApJ, 670, L113

Wakker, B. P., York, D. G., Wilhelm, R., et al. 2008, ApJ, 672, 298

Wakker, B. P., Hernandez, A. K., French, D. M., et al. 2015, ApJ, 814, 40

Wannier, P., \& Wrixon, G. T. 1972, ApJ, 173, L119

Werk, J. K., Prochaska, J. X., Thom, C., et al. 2013, ApJS, 204, 17

Whiting, A. B. 2014, ApJ, 793, 63

Winkel, B., Kalberla, P. M. W., Kerp, J., \& Flöer, L. 2010, ApJS, 188, 488

Winkel, B., Ben Bekhti, N., Darmstädter, V., et al. 2011, A\&A, 533, A105

Wolfe, A. M., \& Prochaska, J. X. 1998, ApJ, 494, L15

Wolfire, M. G., McKee, C. F., Hollenbach, D., \& Tielens, A. G. G. M. 1995, ApJ, 453,673

van Woerden, H., Schwarz, U. J., Peletier, R. F., Wakker, B. P., \& Kalberla, P. M. W. 1999, Nature, 400, 138

Zech, W., Lehner, N., Howk, J. C., Dixon, W., \& Brown, T. 2008, ApJ, 679, 460

Zheng, Y., Putman, M. E., Peek, J. E. G., \& Joung, M. R. 2015, ApJ, 807, 103 
A\&A 607, A48 (2017)

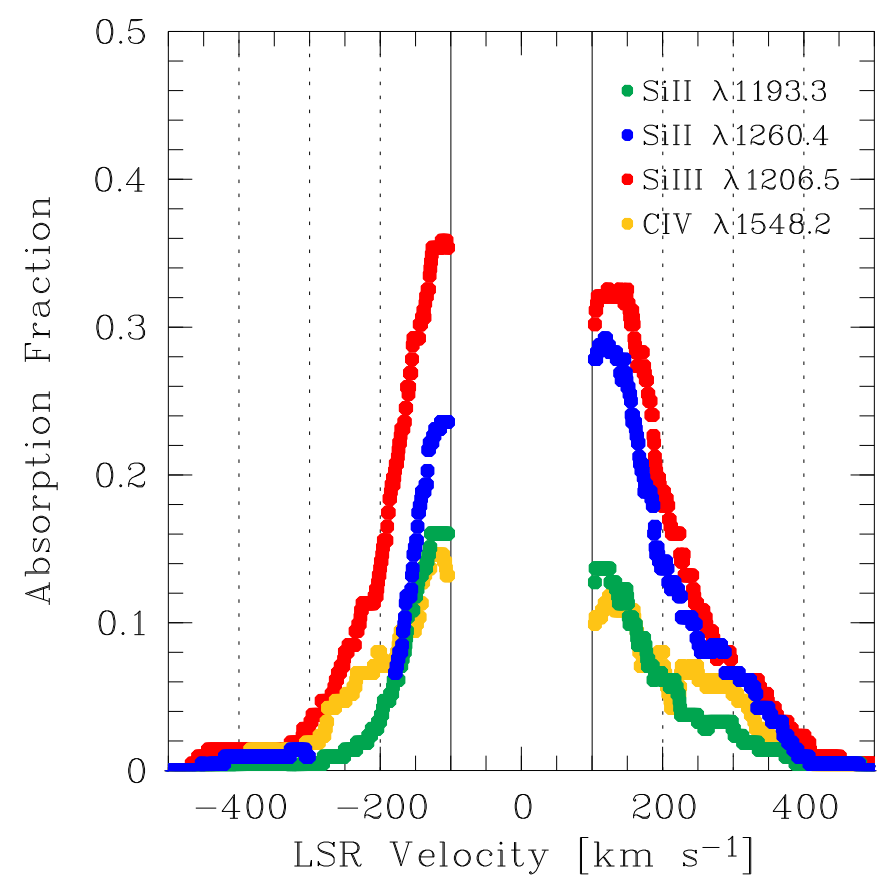

Fig. A.1. Same as Fig. 5, but without considering the area covered by the MS (see Table 3 for adopted $(l, b)$ ranges).

\section{Appendix A: Supplementrary discussions, tables and figures}

\section{A.1. Absorption fraction without MS contribution}

To investigate the role of the MS in the velocity-dependent absorption fraction (Fig. 5) we show in Fig. A.1 the absorption fraction of Si III $\lambda 1206.5$ (red), Si II $\lambda 1260.4$ (blue), Si II $\lambda 1193.3$ (green), and C IV $\lambda 1548.2$ (orange) as a function of LSR velocity excluding the regions covered by the MS (see Table 3). By doing this, the asymmetry between negative and positive velocities (Fig. 5) is reduced, with now almost similar absorption fractions in Si III on both sides of the distribution. This trend demonstrates that the MS and its environment contribute substantially to the absorption fraction of the CGM at high negative velocites.

\section{A.2. Equivalent-width/column-density ratios vs. LSR velocity}

In Fig. A.2 we show the distribution of the same equivalentwidth/column-density ratios as presented in Sect. 4.2, but now as a function of LSR velocity. For this plot we have averaged the equivalent-width ratios over the entire sky in velocity bins of $200 \mathrm{~km} \mathrm{~s}^{-1}$. What can be seen here is that the ratio Si II $\lambda 1260.4 / \mathrm{Si}$ III $\lambda 1206.5$ is small at negative velocities, in particular for $v_{\mathrm{LSR}}<200 \mathrm{~km} \mathrm{~s}^{-1}$. In the same velocity bin the C IV $\lambda 1548.2 / \mathrm{Si}$ III $\lambda 1206.5$ ratio has its maximum, indicating that gas at high negative radial velocities has, on average, a substantially higher degree of ionization than gas at any other (high) radial velocity. Since most of extreme negative velocity absorbers are found in the region, $b<0^{\circ}, l<180^{\circ}$ (see Sect. 4.2), the trends seen in Fig. A.2 are not surprising: they further demonstrate the distinct properties of negative-velocity gas in this direction. In addition, it can be concluded from Fig. A. 2 that the average degree of ionization, as indicated by the above listed equivalent-width/column-density ratios, is higher for CGM gas that moves towards the Sun than for gas that moves away from it.
Table A.1. COS QSO sample.

\begin{tabular}{llrr}
\hline \hline No. & QSO (COS name) & $\begin{array}{r}l \\
{\left[{ }^{\circ}\right]}\end{array}$ & $\begin{array}{r}b \\
{\left[{ }^{\circ}\right]}\end{array}$ \\
\hline 1 & J142947.03+064334.9 & 356.3 & 59.0 \\
2 & RXJ2154.1-4414 & 355.2 & -50.9 \\
3 & 2MASS-J14294076+0321257 & 351.8 & 56.6 \\
4 & PKS2005-489 & 350.4 & -32.6 \\
5 & LBQS-1435-0134 & 348.7 & 51.4 \\
6 & RBS1892 & 345.9 & -58.4 \\
7 & QSO-B1435-0645 & 344.0 & 47.2 \\
8 & VV2006-J140655.7+015713 & 341.8 & 59.0 \\
9 & SDSS-J135726.27+043541.4 & 340.8 & 62.5 \\
10 & IRAS-F22456-5125 & 338.5 & -56.6 \\
11 & ESO-141-55 & 338.2 & -26.7 \\
12 & HE2347-4342 & 336.0 & -69.6 \\
13 & SDSSJ134206.56+050523.8 & 333.9 & 64.9 \\
14 & 1H-2129-624 & 331.1 & -42.5 \\
15 & VV2006-J131545.2+152556 & 329.9 & 77.0 \\
\hline
\end{tabular}

Notes. Full version of Table A.1 is only available in electronic form at the CDS.

\section{A.3. Small-scale structure in HVCs}

As can be seen in Fig. 2, there are two regions in the sky where the projected density of background sources is particularly high: $l=80^{\circ}-110^{\circ}, b=30^{\circ}-60^{\circ}$ (tracing Complex C at $d \approx 10 \mathrm{kpc}$ ) and $l=225^{\circ}-255^{\circ}, b=30^{\circ}-60^{\circ}$ (tracing Complex WA at $d=8-$ $20 \mathrm{kpc}$; Table 3). For these regions/complexes, we constructed equivalent-width maps for the Si II $\lambda 1260.42$ line, as shown in Fig. A.3. On small angular scales $\left(\theta=4^{\circ}\right)$ the equivalent widths vary by factors $2-10$, indicating spatial variations in the column densities of Si II on linear scales of $L \sim 0.7 \mathrm{kpc}$ (Complex C) and $L<1.4 \mathrm{kpc}$ (Complex WA).

Figures 13 and A.3 indicate that the equivalent widths of metal ions can vary substantially within HVCs on sub-kpc scales. The largest differences are seen in the singly-ionized species Si II and C II, while they are typically less pronounced in Si III and C IV. As can be concluded from these figures, the overall velocity structure in the HVC absorption patterns remains mostly unchanged along the different sightlines at the (limited) velocity resolution of the COS instrument $\left(\sim 20 \mathrm{~km} \mathrm{~s}^{-1}\right)$. So far, the information on small-scale structure in HVC gas from absorption-line studies is limited to only a few cloud complexes (e.g., the HVC towards the LMC; Danforth et al. 2002; Bluhm et al. 2001; Lehner et al. 2009; Smoker et al. 2015). In contrast, the presence of pc-scale substructure in HVCs in H I is well established from high-resolution $21 \mathrm{~cm}$ observations (e.g., Wakker et al. 2002; Sembach et al. 2004). The observed variations in the absorption/emission line strengths imply spatial fluctuations in the local gas density and/or changes in physical conditions (temperature, chemical composition, radiation field, etc.). From the few detections of molecular gas in HVCs (Richter et al. 1999, 2001a, 2013 ; Sembach et al. 2001; Wakker 2006; Murray et al. 2015) follows that substantial small-scale structure in the gas densities exists down to sub-pc scales. The formation and maintenance of small-scale structure in the star-less HVCs are based on physical mechanisms that must be different from those in the disk, where supernova explosions and other stellar feedback processes are known to stir up the ISM and form (mostly short-lived) interstellar structures on sub-pc/AU scales (de Avillez \& Breitschwerdt 2007). 
P. Richter et al.: The Milky Way CGM in absorption
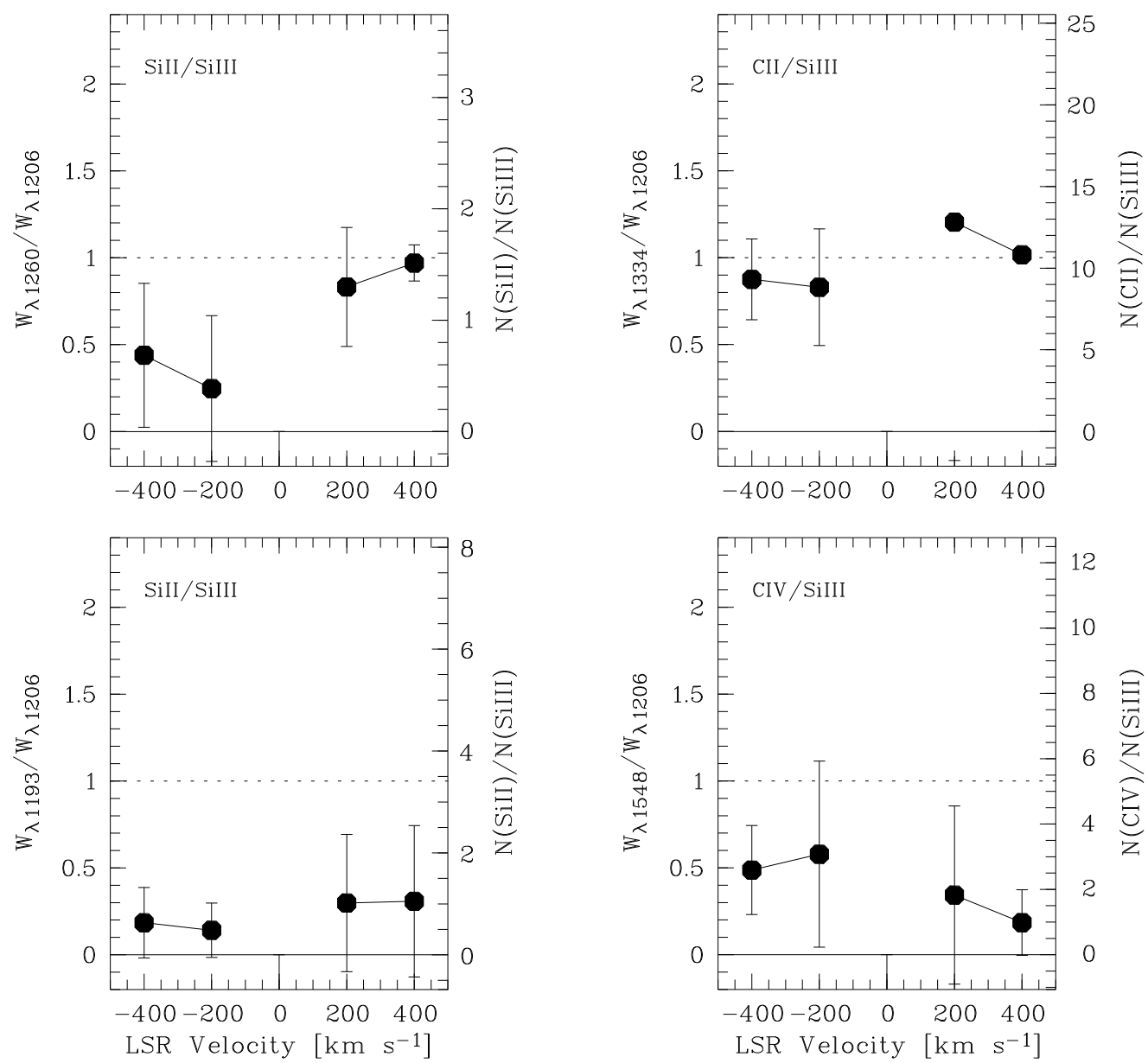

Fig. A.2. Equivalent-width ratios and column density ratios for different transitions of low, intermediate and high ions as a function of LSR velocity. The data are averaged in $200 \mathrm{~km} \mathrm{~s}^{-1}$ wide bins. The error bars indicate the standard deviation from the mean value.
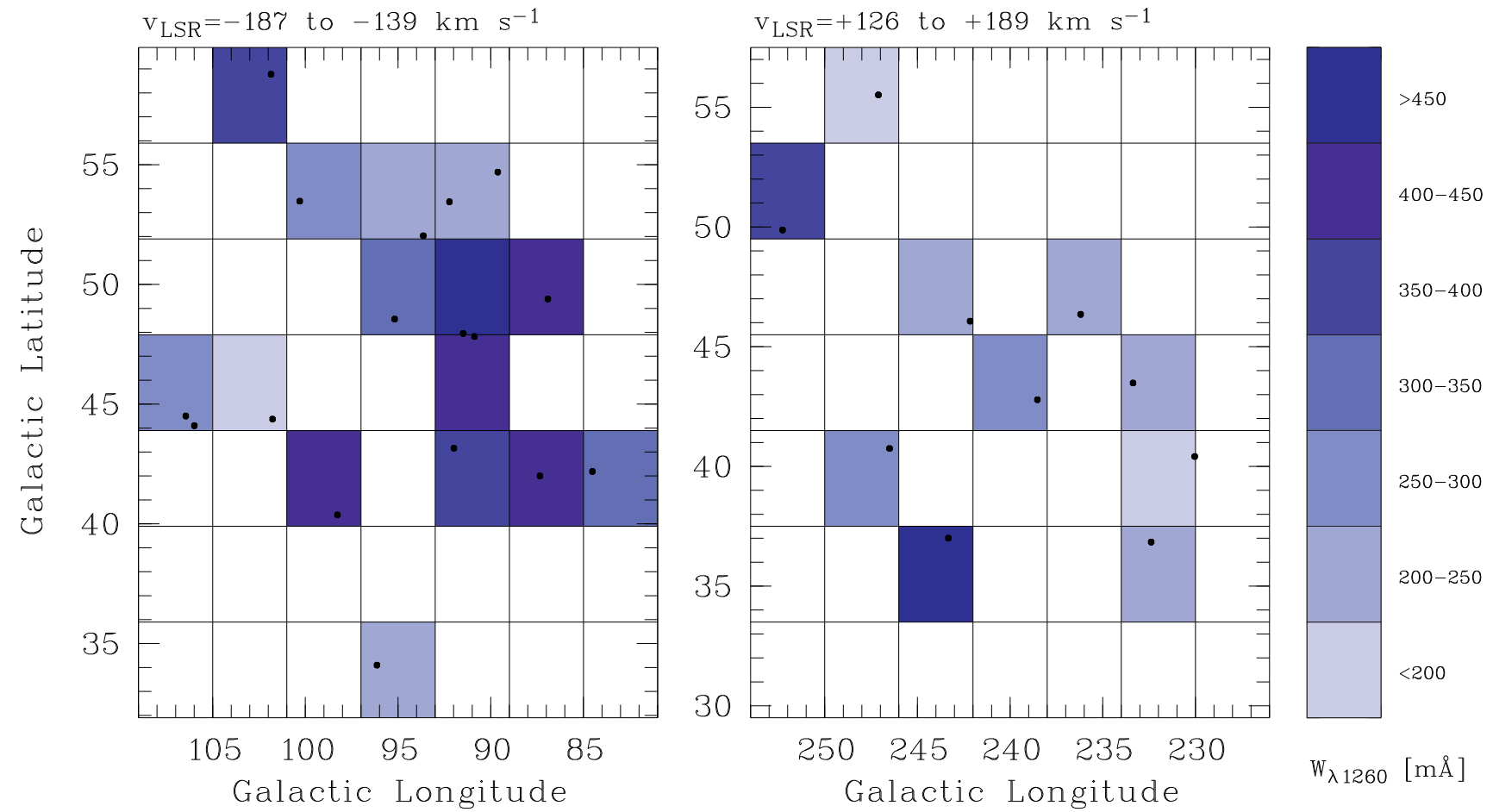

Fig. A.3. Equivalent-width maps for high-velocity Si II $\lambda 1260.4$ absorption for two selected HVC complexes. Left panel: Complex C, right panel: Complex WA. The black dots indicate the positions of the background QSOs. 


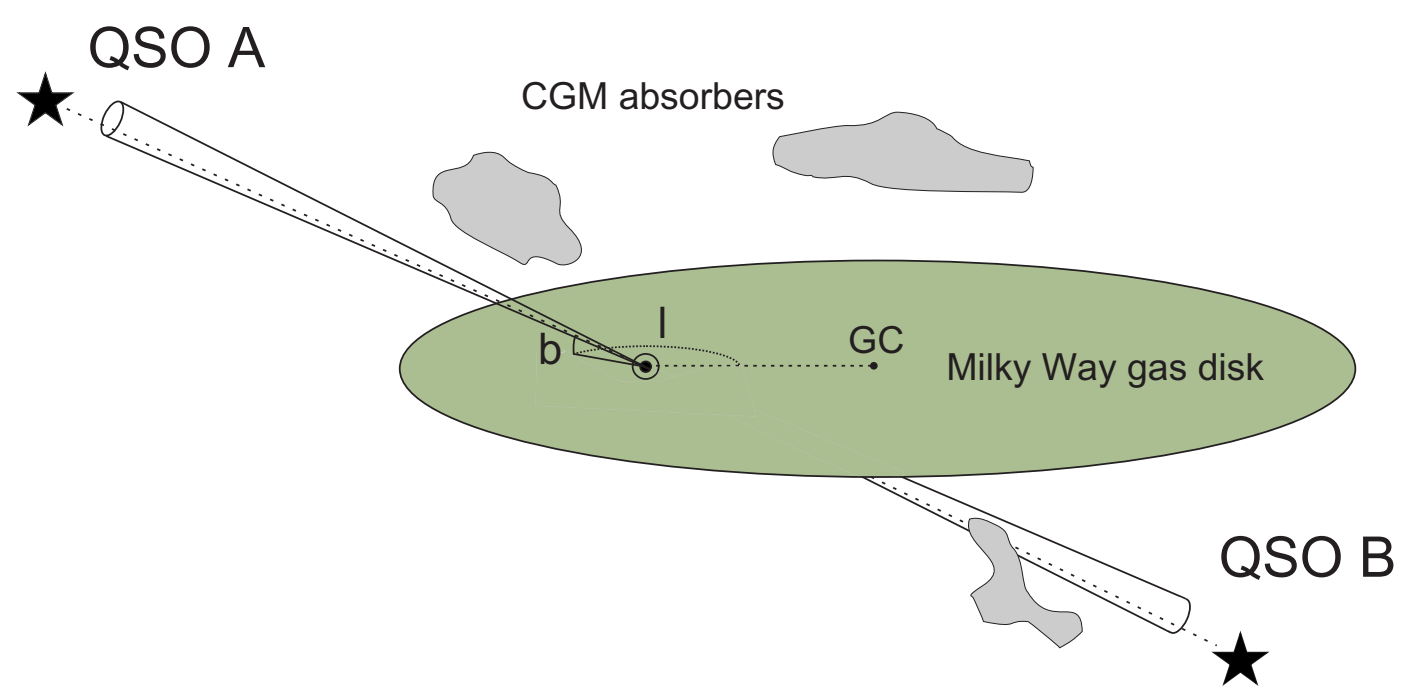

Fig. A.4. Illustration of concept of identifying antipodal QSO sightlines at $\left(l_{1}, b_{1}\right),\left(l_{2}, b_{2}\right)$ passing the Galactic disk and CGM, as seen from an exterior vantage point of the Milky Way. We consider antipodal sightline pairs in cones with an opening angle of $\theta=6^{\circ}$.

Table A.2. Summary of HVC absorption-line measurements.

\begin{tabular}{|c|c|c|c|c|c|c|c|c|c|c|}
\hline No. & QSO (COS name) & Ion & $\begin{array}{r}\lambda_{0} \\
[\AA]]\end{array}$ & $S / N^{a}$ & $\begin{array}{r}v_{\min } \\
{\left[\mathrm{km} \mathrm{s}^{-1}\right]}\end{array}$ & $\begin{array}{r}v_{\max } \\
{\left[\mathrm{km} \mathrm{s}^{-1}\right]}\end{array}$ & $\begin{array}{c}W_{\lambda}^{b} \\
{[\mathrm{~m} \AA]} \\
\end{array}$ & $\begin{array}{r}\Delta W_{\lambda} \\
{[\mathrm{m} \AA]}\end{array}$ & $\log N^{c}$ & $\Delta(\log N)$ \\
\hline 1 & $\mathrm{~J} 142947.03+064334.9$ & CII & 1334.5 & 6 & -155 & -100 & 145 & 27 & $>14.03$ & \\
\hline 2 & $\mathrm{~J} 142947.03+064334.9$ & CIV & 1548.2 & 6 & -165 & -99 & 123 & 35 & 13.77 & 0.13 \\
\hline 3 & $\mathrm{~J} 142947.03+064334.9$ & CIV & 1550.8 & 6 & -131 & -100 & 42 & 22 & 13.55 & 0.16 \\
\hline 4 & $\mathrm{~J} 142947.03+064334.9$ & SilI & 1190.4 & 5 & -133 & -103 & 50 & 19 & 13.17 & 0.20 \\
\hline 5 & $\mathrm{~J} 142947.03+064334.9$ & SilI & 1193.3 & 5 & -149 & -100 & 94 & 26 & $>13.32$ & \\
\hline 6 & $\mathrm{~J} 142947.03+064334.9$ & SiII & 1260.4 & 6 & -155 & -100 & 124 & 23 & $>13.15$ & \\
\hline 7 & $\mathrm{~J} 142947.03+064334.9$ & SiII & 1526.7 & 5 & -142 & -99 & 59 & 32 & 13.64 & 0.26 \\
\hline 8 & $\mathrm{~J} 142947.03+064334.9$ & SiIII & 1206.5 & 4 & -167 & -99 & 122 & 39 & $>12.81$ & \\
\hline 9 & PKS2005-489 & $\mathrm{CII}$ & 1334.5 & 24 & 101 & 202 & 125 & 11 & 13.89 & 0.06 \\
\hline 10 & PKS2005-489 & CIV & 1548.2 & 18 & 101 & 167 & 91 & 13 & 13.50 & 0.10 \\
\hline 11 & PKS2005-489 & SilI & 1193.3 & 25 & 101 & 187 & 68 & 9 & 13.11 & 0.08 \\
\hline 12 & PKS2005-489 & SilI & 1260.4 & 34 & 99 & 206 & 123 & 8 & 13.07 & 0.04 \\
\hline 13 & PKS2005-489 & SiIII & 1206.5 & 25 & 101 & 203 & 143 & 9 & 13.00 & 0.06 \\
\hline 14 & LBQS-1435-013 & CII & 1334.5 & 31 & -136 & -100 & 57 & 4 & 13.59 & 0.07 \\
\hline 15 & LBQS-1435-0134 & CIV & 1548.2 & 35 & -183 & -100 & 118 & 8 & 13.58 & 0.05 \\
\hline 16 & LBQS-1435-0134 & SilI & 1190.4 & 32 & -121 & -100 & & & & \\
\hline 17 & LBQS-1435-0134 & SiII & 1260.4 & 39 & -156 & -100 & 59 & 4 & 12.75 & 0.05 \\
\hline 18 & LBQS-1435-0134 & SiIII & 1206.5 & 35 & -180 & -100 & 49 & 6 & 12.44 & 0.07 \\
\hline
\end{tabular}

Notes. Full version of Table A.2 is only available in electronic form at the CDS. ${ }^{(a)}$ S/N per resolution element. ${ }^{(b)}$ A " $\mathrm{t}$ " in front of the equivalent width indicates a tentative HVC detection. ${ }^{(c)}$ For each ion, the maximum value for $N$ is adopted for the further column-density analysis (see Sect. 2.2). 
Table A.3. Compilation of Local Group galaxies.

\begin{tabular}{|c|c|c|c|c|c|c|c|c|c|c|}
\hline No. & Galaxy name & Alternative name & $\begin{array}{l}\alpha(2000) \\
{[\mathrm{h} \mathrm{m}]}\end{array}$ & $\begin{array}{l}\delta(2000) \\
{\left[{ }^{\circ} \mathrm{m}\right]}\end{array}$ & Type & $\begin{array}{l}d \\
{[\mathrm{kpc}]}\end{array}$ & $\begin{array}{r}M_{V} \\
{[\mathrm{mag}]}\end{array}$ & $\begin{array}{r}v_{\mathrm{rad}} \\
{\left[\mathrm{km} \mathrm{s}^{-1}\right]} \\
\end{array}$ & $\begin{array}{r}l \\
{\left[{ }^{\circ}\right]} \\
\end{array}$ & $\begin{array}{r}b \\
{\left[{ }^{\circ}\right]} \\
\end{array}$ \\
\hline 1 & M31 & NGC 224 & 0040.0 & +4059 & $\mathrm{Sb}$ & 770 & -21.1 & -299 & 121.2 & -21.6 \\
\hline 2 & M33 & NGC 598 & 0131.1 & +3024 & $\mathrm{Sc}$ & 850 & -18.9 & -180 & 133.6 & -31.3 \\
\hline 3 & LMC & & 0524.0 & -6948 & Irr & 49 & -18.1 & 270 & 280.5 & -32.9 \\
\hline 4 & IC 10 & & 0017.7 & +5901 & Irr & 660 & -17.6 & -343 & 119.0 & -3.3 \\
\hline 5 & NGC 6822 & DDO 209 & 1942.1 & -1456 & Irr & 540 & -16.4 & -49 & 25.3 & -18.4 \\
\hline 6 & M32 & NGC 221 & 0040.0 & +4036 & $\mathrm{E} 2$ & 770 & -16.4 & -205 & 121.2 & -22.0 \\
\hline 7 & NGC 205 & & 0037.6 & +4125 & E5 & 830 & -16.3 & -239 & 120.7 & -21.7 \\
\hline 8 & $\mathrm{SMC}$ & & 0051.0 & -7306 & Irr & 58 & -16.2 & 163 & 302.8 & -44.3 \\
\hline 9 & NGC 3109 & DDO 236 & 1000.8 & -2555 & Irr & 1260 & -15.8 & 403 & 262.1 & +23.1 \\
\hline 10 & NGC 185 & & 0036.2 & +4804 & E3 & 620 & -15.3 & -208 & 120.8 & -14.5 \\
\hline 11 & IC 1613 & DDO 8 & 0102.2 & +0151 & Irr & 715 & -14.9 & -236 & 129.8 & -60.6 \\
\hline 12 & NGC 147 & DDO 3 & 0030.5 & +4814 & $\mathrm{E} 4$ & 755 & -14.8 & -193 & 119.8 & -14.3 \\
\hline 13 & Sextans A & DDO 75 & 1008.6 & -0428 & Irr & 1450 & -14.4 & 325 & 246.2 & +39.9 \\
\hline 14 & Sextans B & DDO 70 & 0957.4 & +0534 & Irr & 1300 & -14.3 & 301 & 233.2 & +43.8 \\
\hline 15 & WLM & DDO 221 & 2359.4 & -1545 & Irr & 940 & -14.0 & -116 & 75.9 & -73.6 \\
\hline 16 & Sagittarius & & 1851.9 & -3030 & $\mathrm{dE7}$ & 24 & -14.0 & 140 & 5.6 & -14.1 \\
\hline 17 & Fornax & & 0237.8 & -3444 & $\mathrm{dE} 3$ & 131 & -13.0 & 53 & 237.1 & -65.7 \\
\hline 18 & Pegasus & DDO 216 & 2326.1 & +1428 & Irr & 760 & -12.7 & -181 & 94.8 & -43.5 \\
\hline 19 & And VII & Cas Dw & 2324.1 & +5025 & $\mathrm{dE} 3$ & 760 & -12.0 & -307 & 109.5 & -10.0 \\
\hline 20 & Leo I & DDO 74 & 1005.8 & +1233 & $\mathrm{dE} 3$ & 251 & -12.0 & 285 & 226.0 & +49.1 \\
\hline 21 & Leo A & DDO 69 & 0956.5 & +3059 & Irr & 692 & -11.7 & 26 & 196.9 & +52.4 \\
\hline 22 & And II & & 0113.5 & +3309 & $\mathrm{dE} 3$ & 680 & -11.7 & -188 & 128.9 & -29.2 \\
\hline 23 & And I & & 0043.0 & +3744 & $\mathrm{dE} 0$ & 790 & -11.7 & -380 & 121.7 & -24.9 \\
\hline 24 & And VI & Peg Dw & 2349.2 & +2418 & $\mathrm{dE} 3$ & 815 & -11.3 & -354 & 106.1 & -36.3 \\
\hline 25 & SagDIG & & 1927.9 & -1747 & Irr & 1150 & -11.0 & -79 & 21.1 & -16.3 \\
\hline 26 & Aquarius & DDO 210 & 2044.2 & -1301 & Irr & 950 & -10.9 & -131 & 34.0 & -31.3 \\
\hline 27 & Antlia & & 1001.8 & -2705 & $\mathrm{dE} 3$ & 1150 & -10.7 & 361 & 263.1 & +22.3 \\
\hline 28 & Sculptor & & 0057.6 & -3358 & $\mathrm{dE}$ & 78 & -10.7 & 107 & 287.5 & -83.2 \\
\hline 29 & And III & & 0032.6 & +3612 & dE6 & 760 & -10.2 & -355 & 119.3 & -26.2 \\
\hline 30 & Leo II & DDO 93 & 1110.8 & +2226 & $\mathrm{dE} 0$ & 230 & -10.2 & 76 & 220.2 & +67.2 \\
\hline 31 & Cetus & & 0023.6 & -1119 & $\mathrm{dE} 4$ & 800 & -10.1 & -87 & 101.4 & -72.8 \\
\hline 32 & Sextans & & 1010.6 & -0124 & $\mathrm{dE} 4$ & 90 & -10.0 & 224 & 243.4 & +42.2 \\
\hline 33 & Phoenix & & 0149.0 & -4442 & Irr & 390 & -9.9 & 56 & 272.2 & -68.9 \\
\hline 34 & LGS 3 & & 0101.2 & +2137 & $\mathrm{Irr} / \mathrm{dE}$ & 810 & -9.7 & -277 & 126.8 & -40.9 \\
\hline 35 & Tucana & & 2238.5 & -6441 & dE5 & 900 & -9.6 & 182 & 322.9 & -47.4 \\
\hline 36 & Carina & & 0640.4 & -5055 & $\mathrm{dE} 4$ & 87 & -9.2 & 223 & 260.1 & -22.2 \\
\hline 37 & And V & & 0107.3 & +4722 & $\mathrm{dE}$ & 810 & -9.1 & -403 & 126.2 & -15.1 \\
\hline 38 & Ursa Minor & DDO 199 & 1508.2 & +6723 & $\mathrm{dE} 5$ & 69 & -8.9 & -250 & 105.0 & +44.8 \\
\hline 39 & Draco & DDO 228 & 1719.2 & +5758 & $\mathrm{dE} 3$ & 76 & -8.6 & -289 & 86.4 & +34.7 \\
\hline 40 & And IX & & 0050.0 & +4257 & $\mathrm{dE}$ & 780 & -8.3 & -208 & 123.2 & -19.7 \\
\hline
\end{tabular}


A\&A 607, A48 (2017)

Table A.4. Neutral/total hydrogen column densities and neutral gas fractions in HVC absorbers.

\begin{tabular}{llrrrr}
\hline \hline No. & QSO & $\begin{array}{r}\left\langle v_{\mathrm{HVC}}\right\rangle \\
{\left[\mathrm{km} \mathrm{s}^{-1}\right]}\end{array}$ & $\log N(\mathrm{HI})$ & $\log N(\mathrm{H})$ & $\log f_{\mathrm{HI}}$ \\
\hline 1 & J142947.03+064334.9 & -133 & $<18.70$ & $>19.19$ & $<-0.49$ \\
2 & PKS2005-489 & 152 & $<18.70$ & 18.85 & $<-0.15$ \\
3 & LBQS-1435-0134 & -140 & $<18.70$ & 18.46 & $<0.24$ \\
4 & QSO-B1435-0645 & -119 & $<18.70$ & 18.55 & $<0.15$ \\
5 & VV2006-J140655.7+015713 & -131 & $<18.70$ & 18.55 & $<0.15$ \\
6 & SDSS-J135726.27+043541.4 & -116 & $<18.70$ & $>18.62$ & $<0.08$ \\
7 & IRAS-F22456-5125 & 146 & $<18.70$ & 18.23 & $<0.47$ \\
8 & SDSSJ134206.56+050523.8 & -106 & $<18.70$ & 17.86 & $<0.84$ \\
9 & 1H-2129-624 & 186 & $<18.70$ & 18.62 & $<0.08$ \\
10 & 1H-2129-624 & 129 & $<18.70$ & 18.31 & $<0.39$ \\
11 & SDSSJ134251.60-005345.3 & -114 & $<18.70$ & 18.94 & $<-0.24$ \\
12 & HARO11 & 142 & 18.94 & 18.94 & $<0.00$ \\
13 & HARO11 & -164 & 18.92 & $>19.16$ & $<-0.22$ \\
14 & RXS-J00057-5007 & 130 & $<18.70$ & $>19.19$ & $<-0.49$ \\
15 & RBS144 & 156 & 19.77 & 19.77 & 0.00 \\
\hline
\end{tabular}

Notes. Full version of Table A.4 is only available in electronic form at the CDS. 
Table A.5. Antipodal sightline pairs.

\begin{tabular}{|c|c|c|c|c|}
\hline Pair no. & QSO & $\begin{array}{r}l \\
{\left[^{\circ}\right]}\end{array}$ & $\begin{array}{r}b \\
{\left[^{\circ}\right]}\end{array}$ & HVC complex \\
\hline 1 & 2MASS-J14294076+0321257 & 351.8 & 56.6 & Complex L \\
\hline 1 & HB89-0232-042 & 174.5 & -56.2 & \\
\hline 2 & PKS2005-489 & 350.4 & -32.6 & Magellanic Stream \\
\hline 2 & SDSSJ080908.13+461925.6 & 173.3 & 32.3 & Complex A \\
\hline 3 & QSO-B1435-0645 & 344.0 & 47.2 & Complex L \\
\hline 3 & MRK595 & 164.8 & -46.5 & \\
\hline 4 & IRAS-F22456-5125 & 338.5 & -56.6 & Magellanic Stream \\
\hline 4 & $1 \mathrm{ES} 1028+511$ & 161.4 & 54.4 & Complex M \\
\hline 5 & IRAS-F22456-5125 & 338.5 & -56.6 & Magellanic Stream \\
\hline 5 & 1SAXJ1032.3+5051 & 161.4 & 54.6 & Complex M \\
\hline 6 & $1 \mathrm{H}-2129-624$ & 331.1 & -42.5 & Magellanic Stream \\
\hline 6 & SDSSJ092837.98+602521.0 & 154.1 & 42.4 & Complex A \\
\hline 7 & VV2006-J130524.3+035731 & 311.7 & 66.6 & \\
\hline 7 & LBQS-0107-0235 & 134.0 & -64.8 & Magellanic Stream \\
\hline 8 & UKS-0242-724 & 291.8 & -42.4 & Magellanic Stream \\
\hline 8 & SDSSJ145907.58+714019.9 & 110.0 & 42.1 & Complex C \\
\hline 9 & ESO-031-G-008 & 290.3 & -40.8 & Magellanic Stream \\
\hline 9 & SDSSJ145907.58+714019.9 & 110.0 & 42.1 & Complex C \\
\hline 10 & HE1159-1338 & 285.1 & 47.2 & \\
\hline 10 & PG0003+158 & 107.3 & -45.3 & Magellanic Stream \\
\hline 11 & CAL-F-COPY & 277.2 & -35.4 & Magellanic Stream \\
\hline 11 & SDSSJ171737.95+655939.3 & 96.1 & 34.1 & Complex C \\
\hline 12 & CAL-F-COPY & 277.2 & -35.4 & Magellanic Stream \\
\hline 12 & HS1700+6416 & 94.4 & 36.2 & Complex C \\
\hline 13 & IRAS-L06229-6434 & 274.3 & -27.3 & Magellanic Stream \\
\hline 13 & $\mathrm{H} 1821+643$ & 94.0 & 27.4 & Complex C \\
\hline 14 & QSO-B1126-041 & 267.6 & 52.7 & \\
\hline 14 & NGC7714 & 88.2 & -55.6 & Magellanic Stream \\
\hline 15 & IRAS-F04250-5718 & 267.0 & -42.0 & Magellanic Stream \\
\hline 15 & SBS $1624+575$ & 87.3 & 42.0 & Complex C \\
\hline 16 & IRAS-F04250-5718 & 267.0 & -42.0 & Magellanic Stream \\
\hline 16 & PG1626+554 & 84.5 & 42.2 & Complex C \\
\hline 17 & 2DFGRSS393Z082 & 226.6 & -65.0 & Magellanic Stream \\
\hline 17 & SDSS-J145424.33+304658.3 & 48.1 & 62.9 & \\
\hline 18 & SDSSJ094733.21+100508.7 & 225.4 & 43.5 & Complex WAWB \\
\hline 18 & PHL1811 & 47.5 & -44.8 & Complex GCN \\
\hline 19 & TONS210 & 225.0 & -83.2 & Magellanic Stream \\
\hline 19 & SDSSJ133045.15+281321.4 & 42.4 & 81.2 & \\
\hline 20 & PKS0405-123 & 204.9 & -41.8 & \\
\hline 20 & SDSS-J160519.70+144852.2 & 27.8 & 43.4 & \\
\hline 21 & PKS0405-123 & 204.9 & -41.8 & \\
\hline 21 & $1 \mathrm{ES} 1553+113$ & 21.9 & 44.0 & \\
\hline 22 & BZBJ1001+2911 fb01-1.pdf & 199.5 & 52.6 & Complex M \\
\hline 22 & PKS2155-304 & 17.7 & -52.2 & Complex GCN \\
\hline 23 & FBQSJ1010+3003 & 198.4 & 54.6 & Complex M \\
\hline 23 & PKS2155-304 & 17.7 & -52.2 & Complex GCN \\
\hline 24 & 2MASS-J09591486+3203573 & 194.7 & 52.5 & Complex M \\
\hline 24 & PKS2155-304 & 17.7 & -52.2 & Complex GCN \\
\hline
\end{tabular}




\section{Appendix B: Sightline pairs and HVC finding charts}
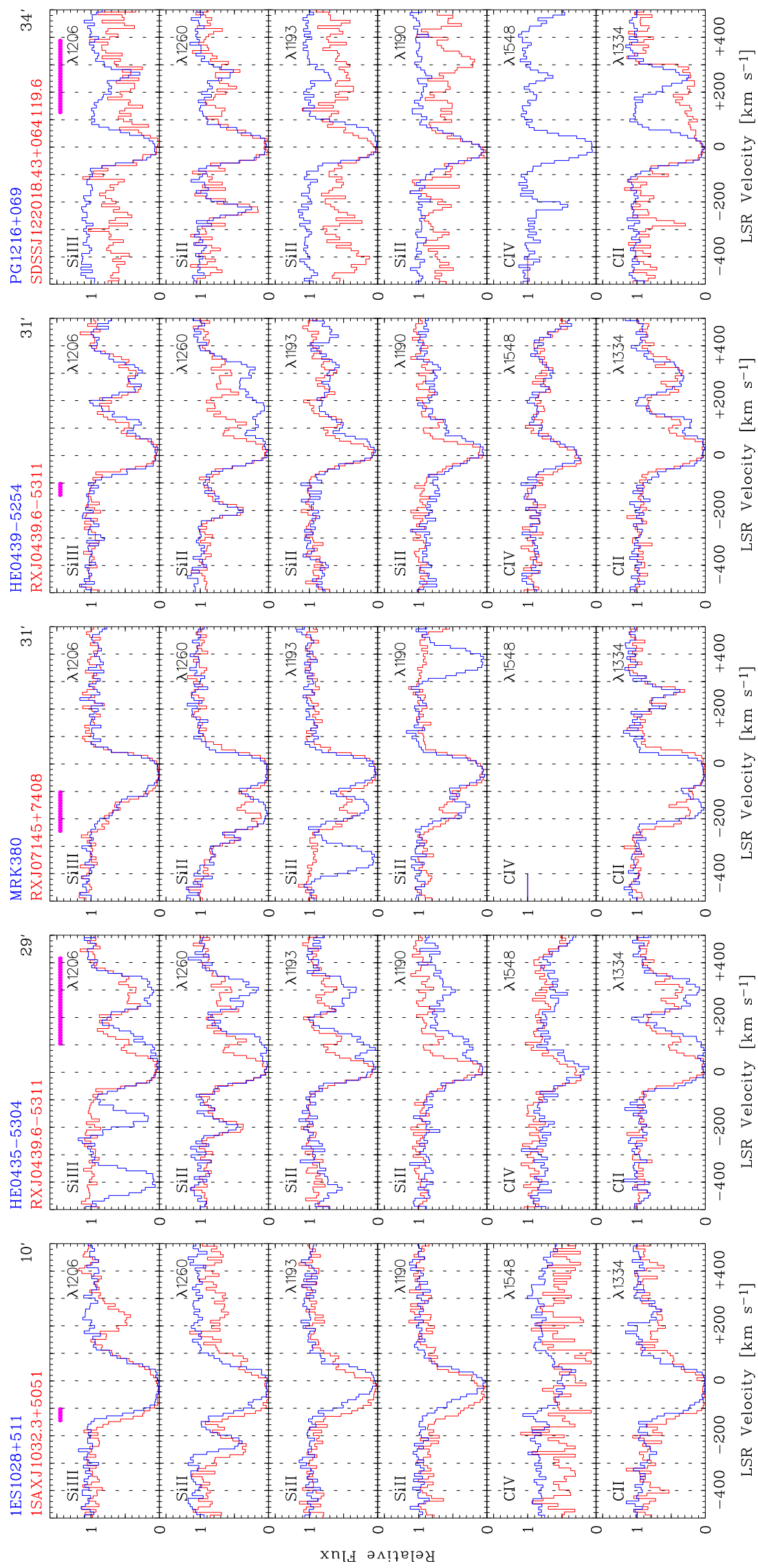

Fig. B.1. Full set of velocity plots for all 12 sightline pairs with angular separations $\leq 1 \mathrm{deg}$ (see Fig. 13 for a detailed description). 
P. Richter et al.: The Milky Way CGM in absorption
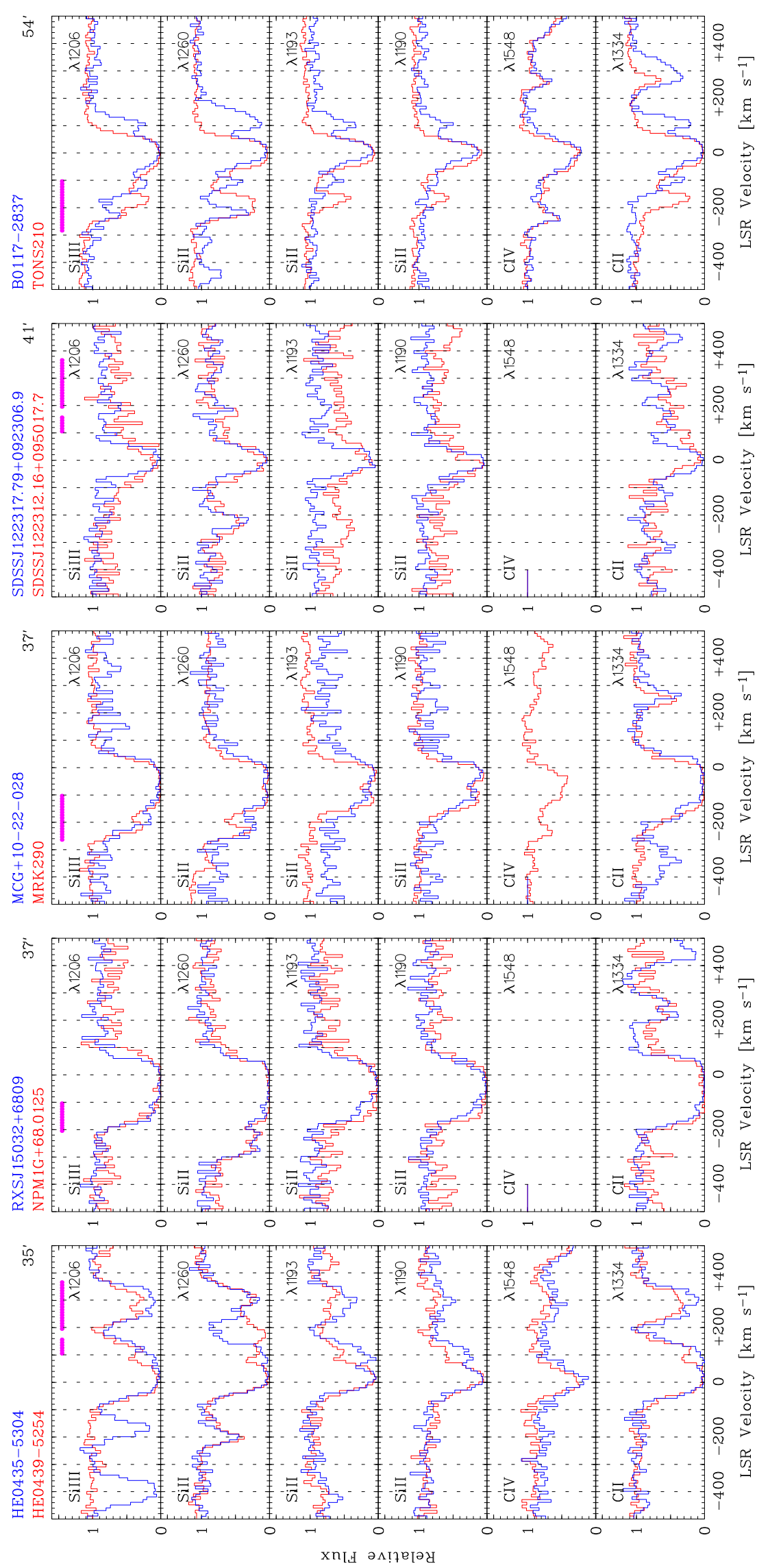

Fig. B.1. continued. 
A\&A 607, A48 (2017)
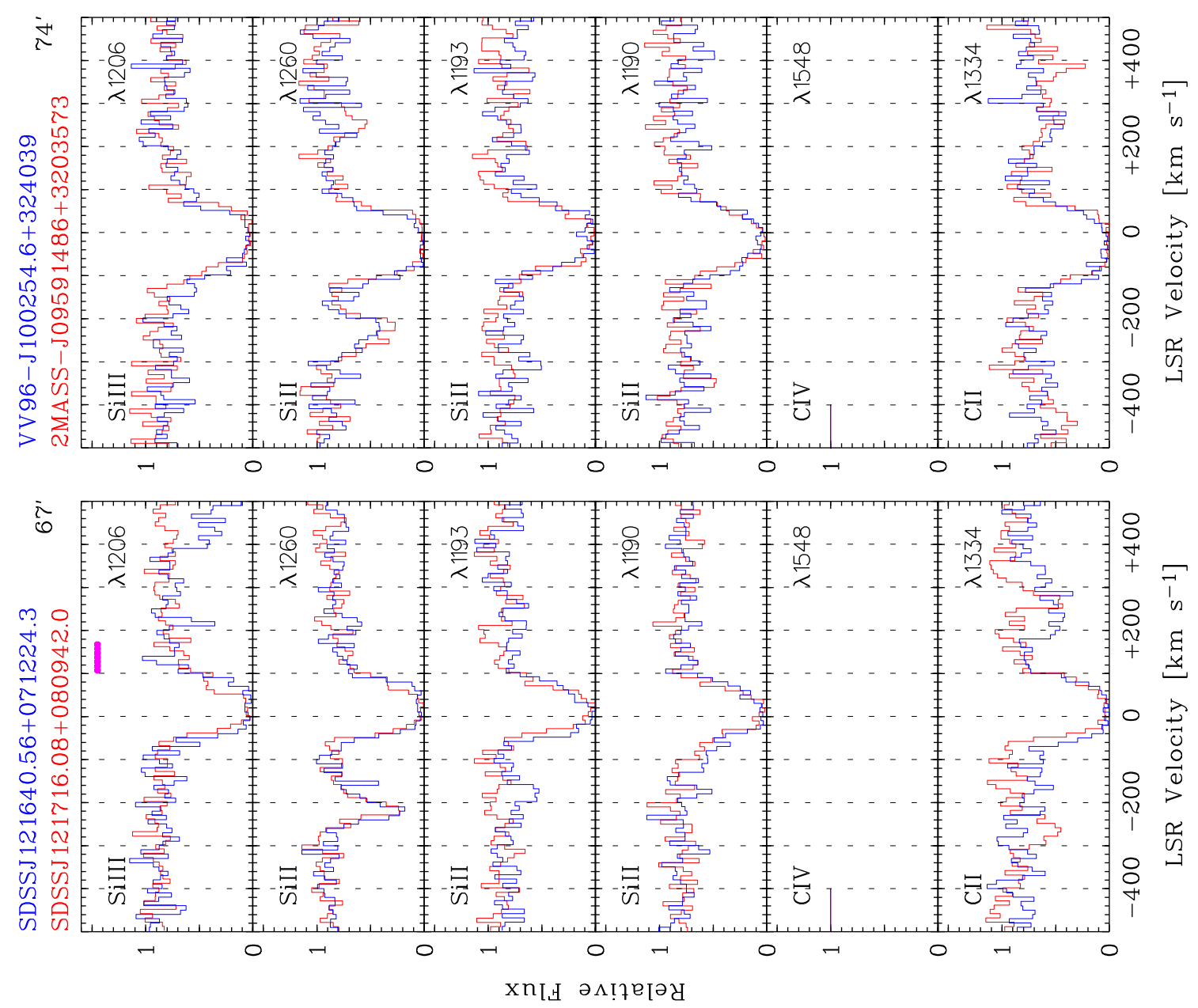

Fig. B.1. continued. 
P. Richter et al.: The Milky Way CGM in absorption
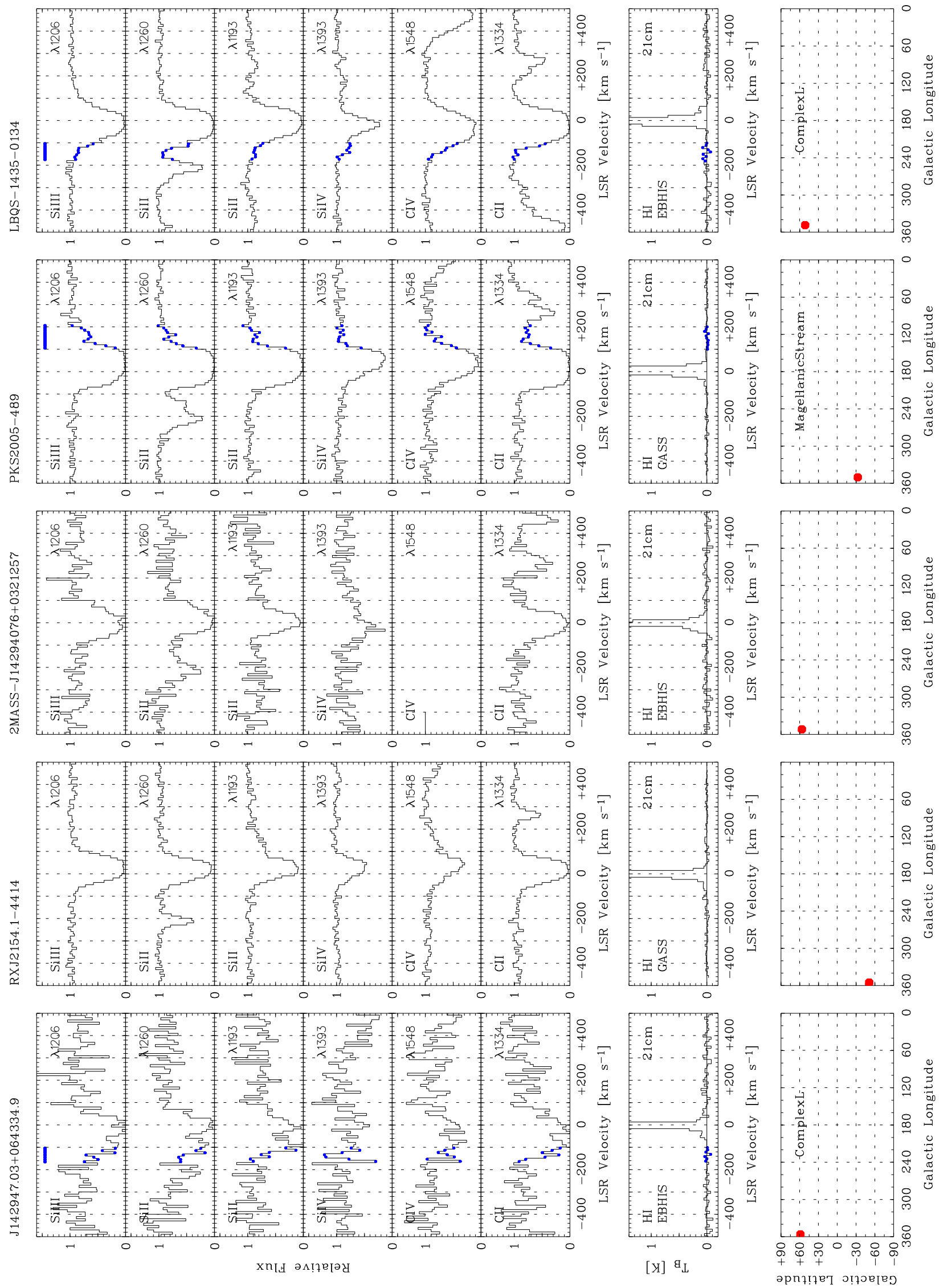

Fig. B.2. Full set for HVC finding charts for all 270 sightlines in our survey (see Fig. 1 for a detailed description). LG galaxies close to the sightlines have been indicated with the red ID numbers (Table A.3, first row; Sect. 5.3) and red tic marks in the upper panels. Note that the continuum shown here (and in Fig. 1) represents the global continuum derived from the automated continuum fitting procedure (Sect. 2.2), but not the exact continuum that was used for the measurements. 

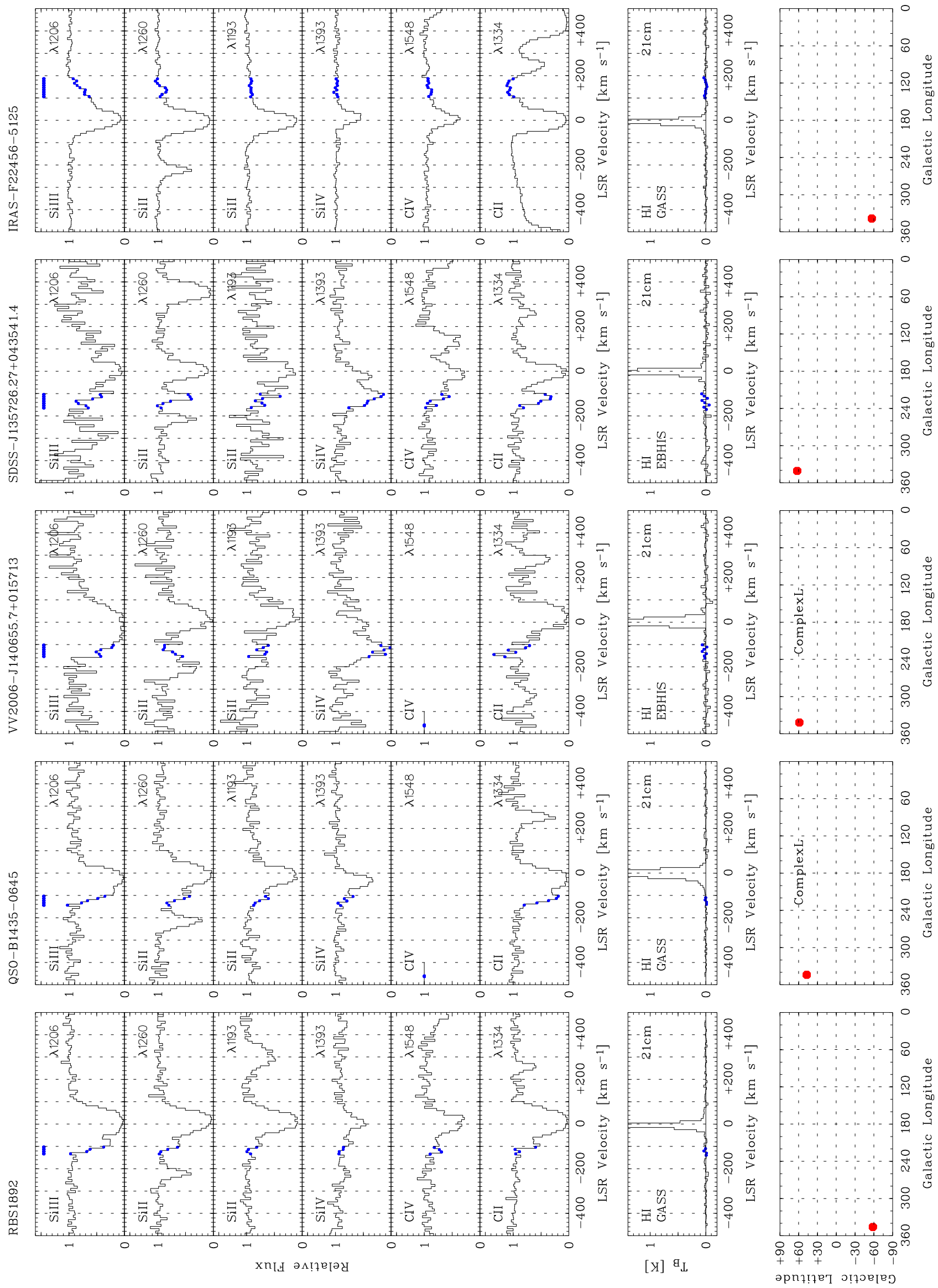

Fig. B.2. continued. 
P. Richter et al.: The Milky Way CGM in absorption
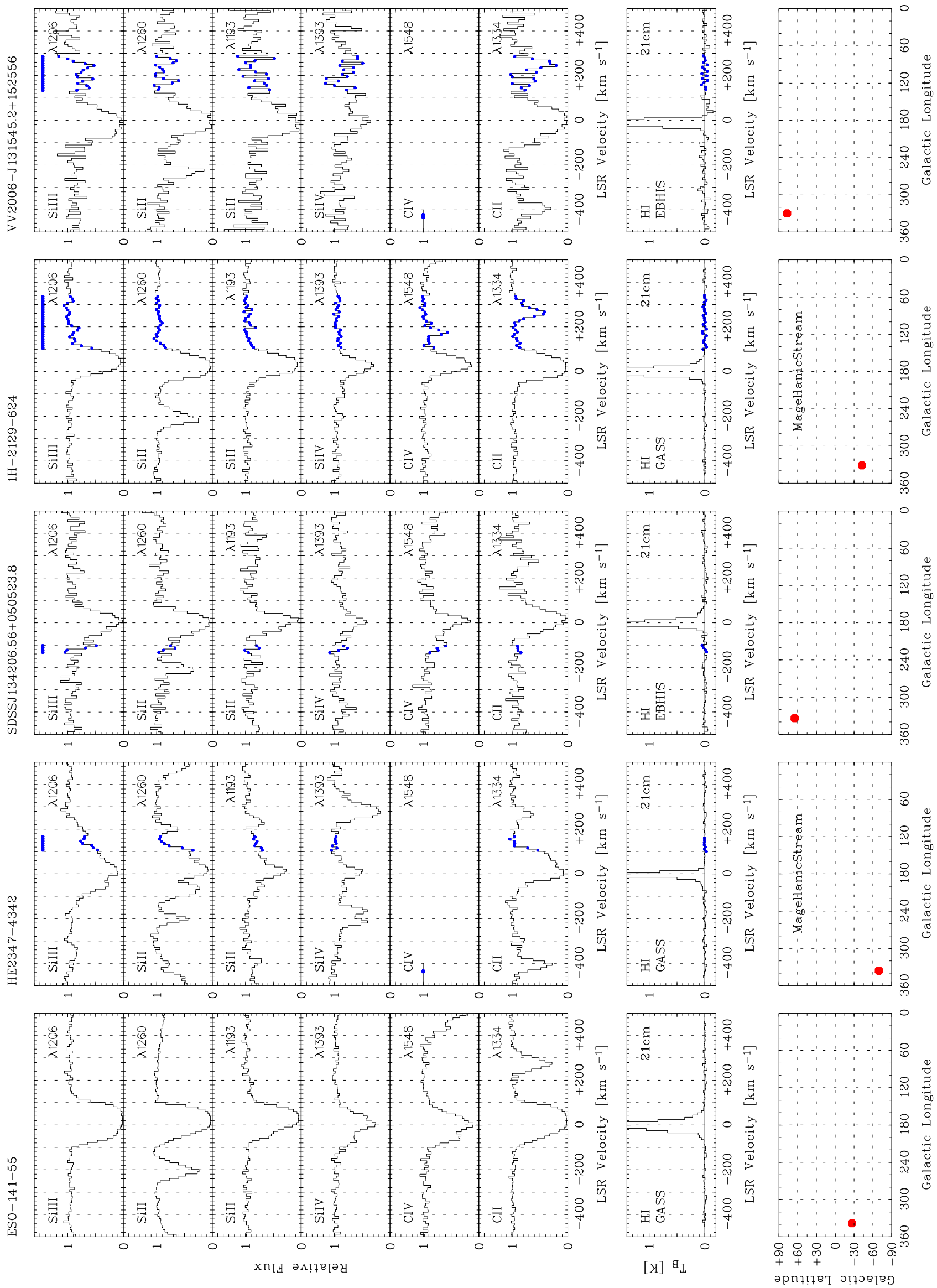

Fig. B.2. continued. 

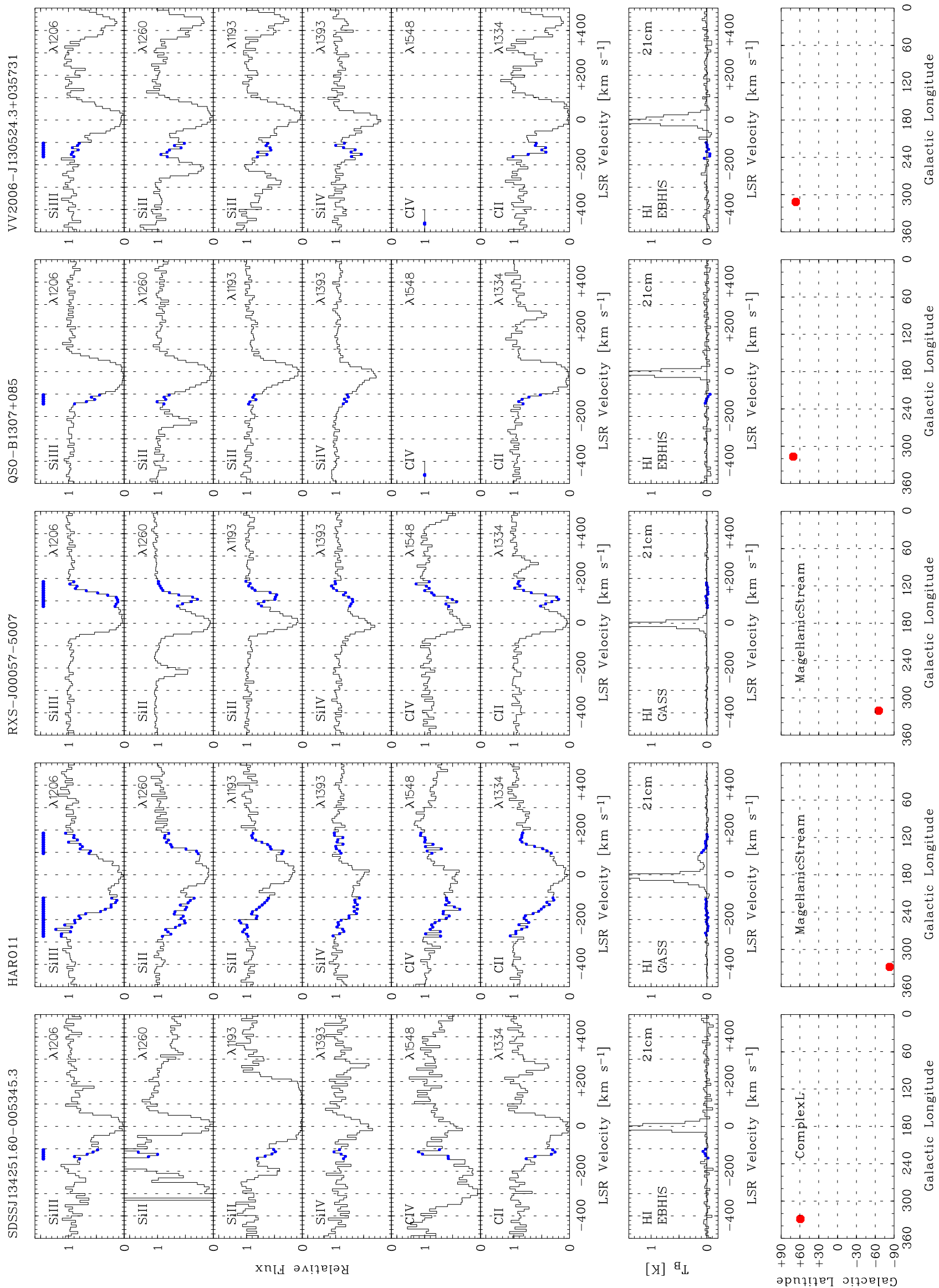

Fig. B.2. continued. 
P. Richter et al.: The Milky Way CGM in absorption
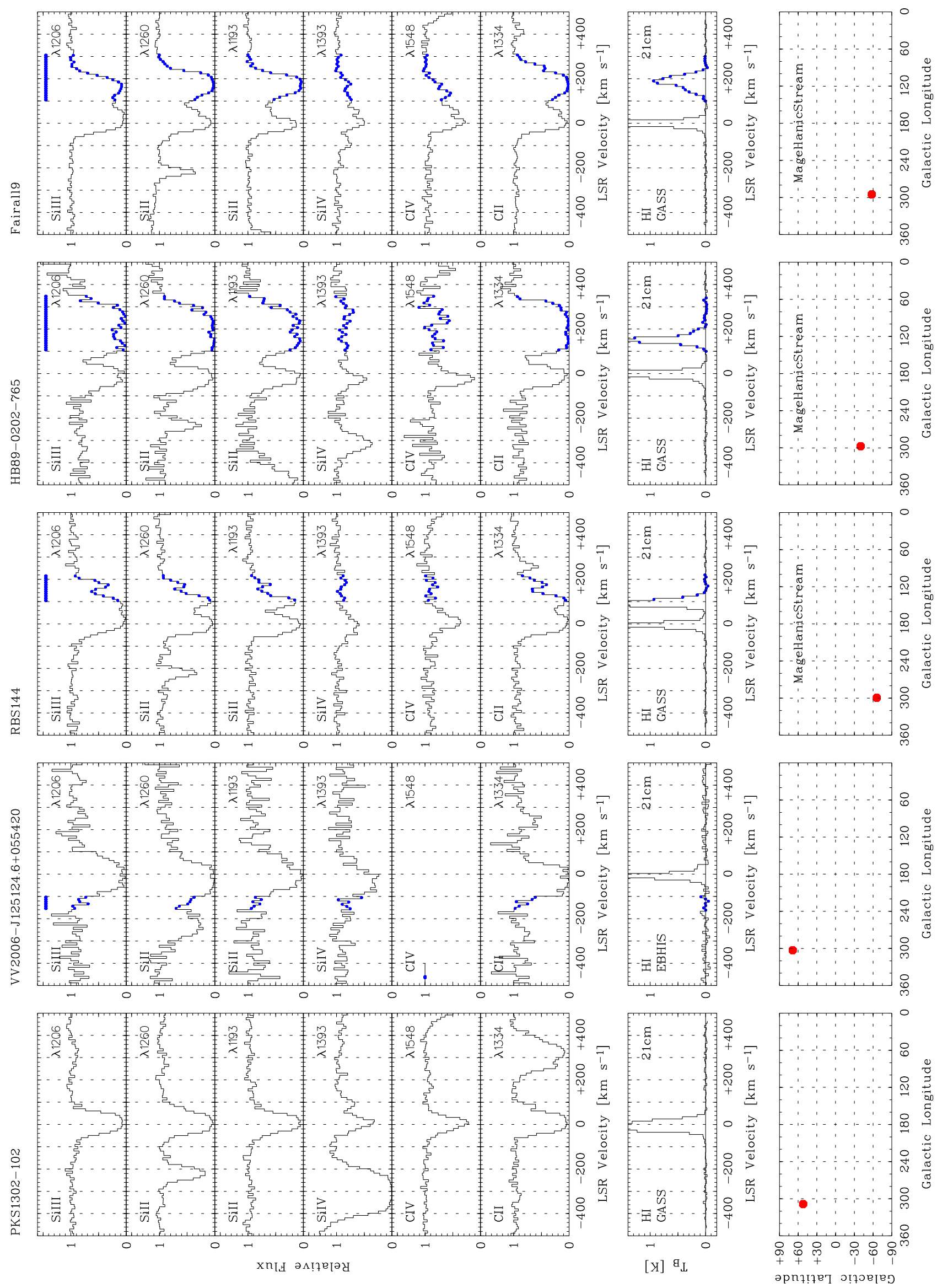

Fig. B.2. continued. 

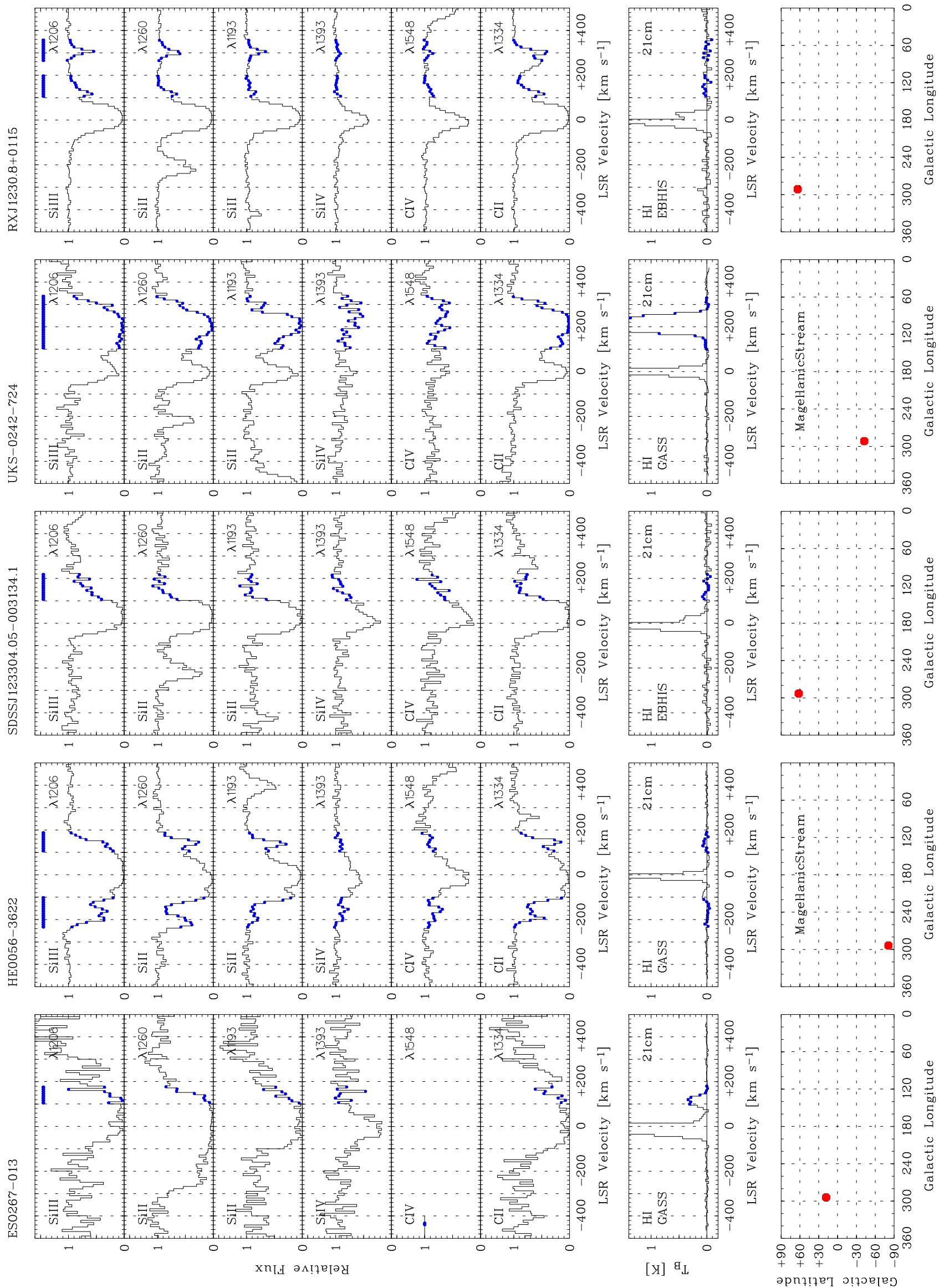

Fig. B.2. continued. 
P. Richter et al.: The Milky Way CGM in absorption


Fig. B.2. continued. 

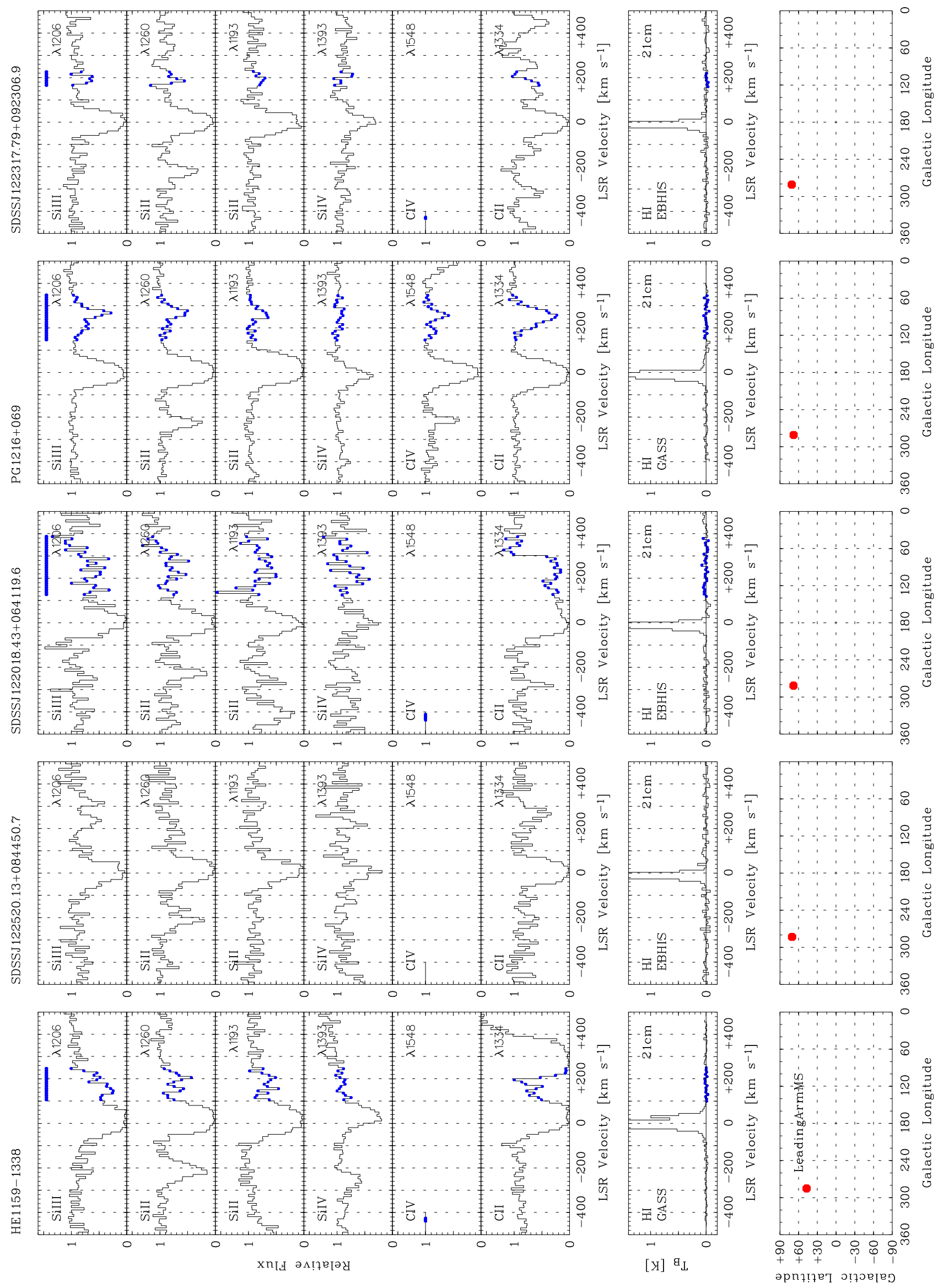

Fig. B.2. continued. 
P. Richter et al.: The Milky Way CGM in absorption
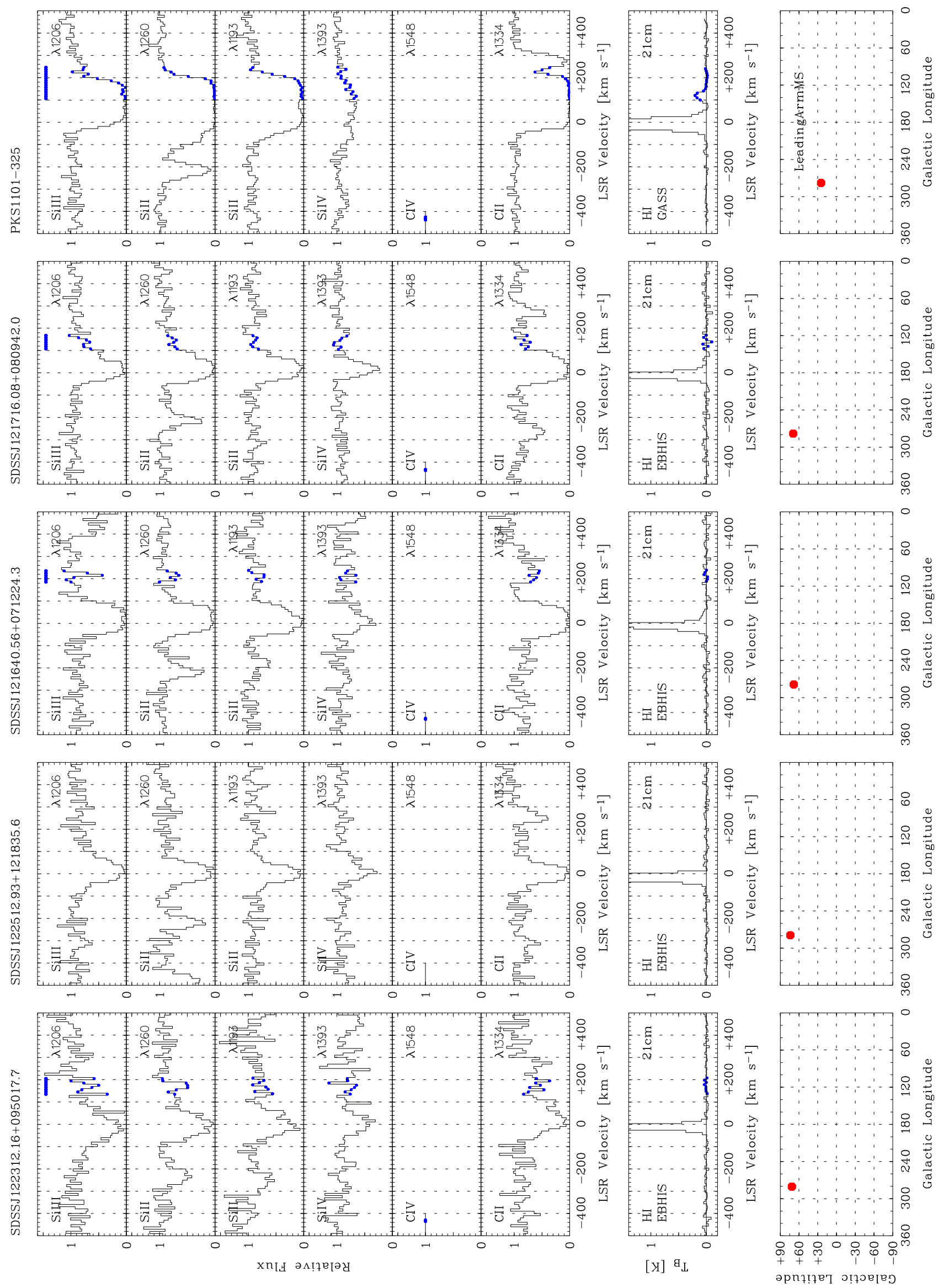

Fig. B.2. continued. 

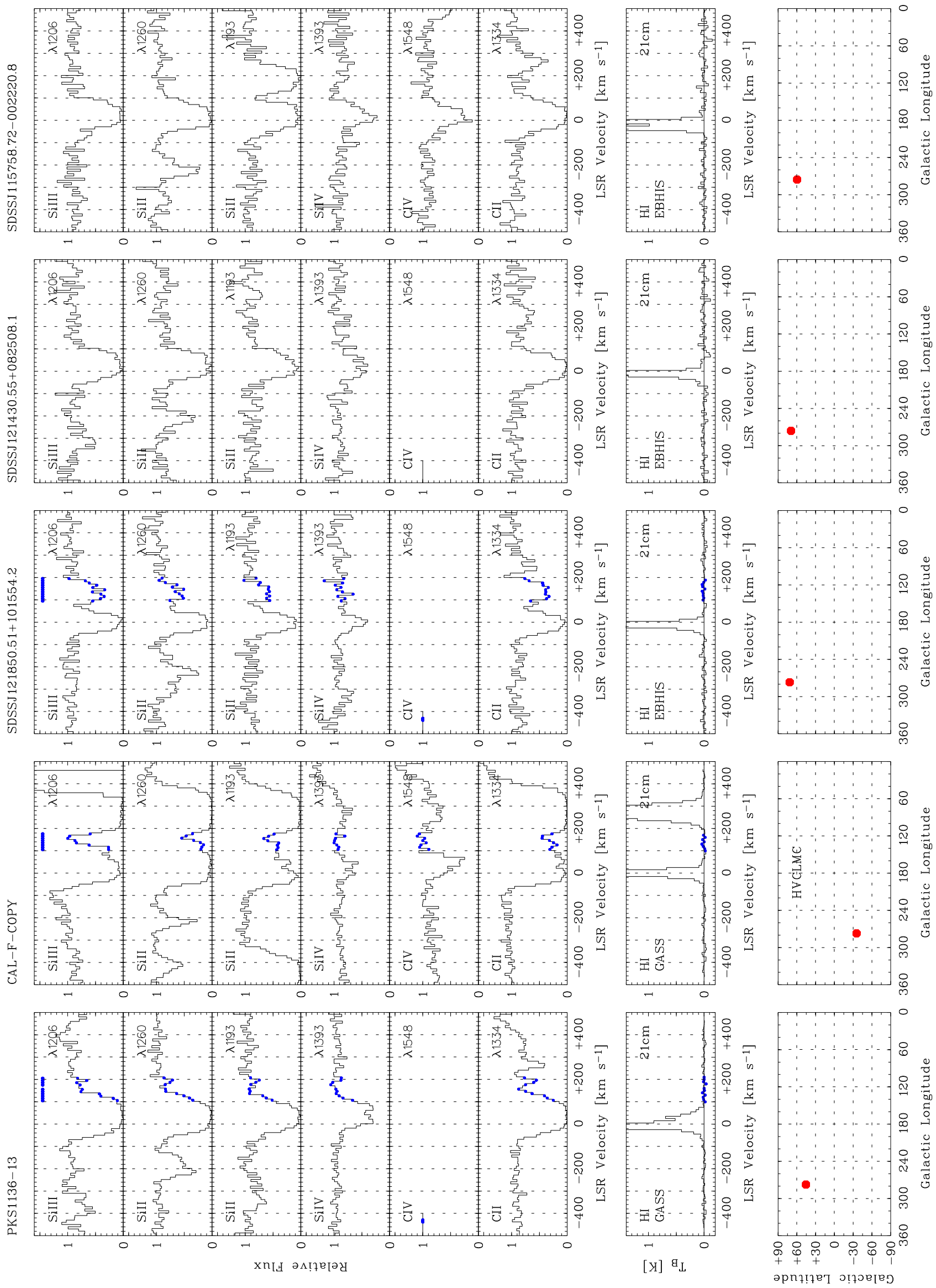

Fig. B.2. continued. 
P. Richter et al.: The Milky Way CGM in absorption


Fig. B.2. continued. 

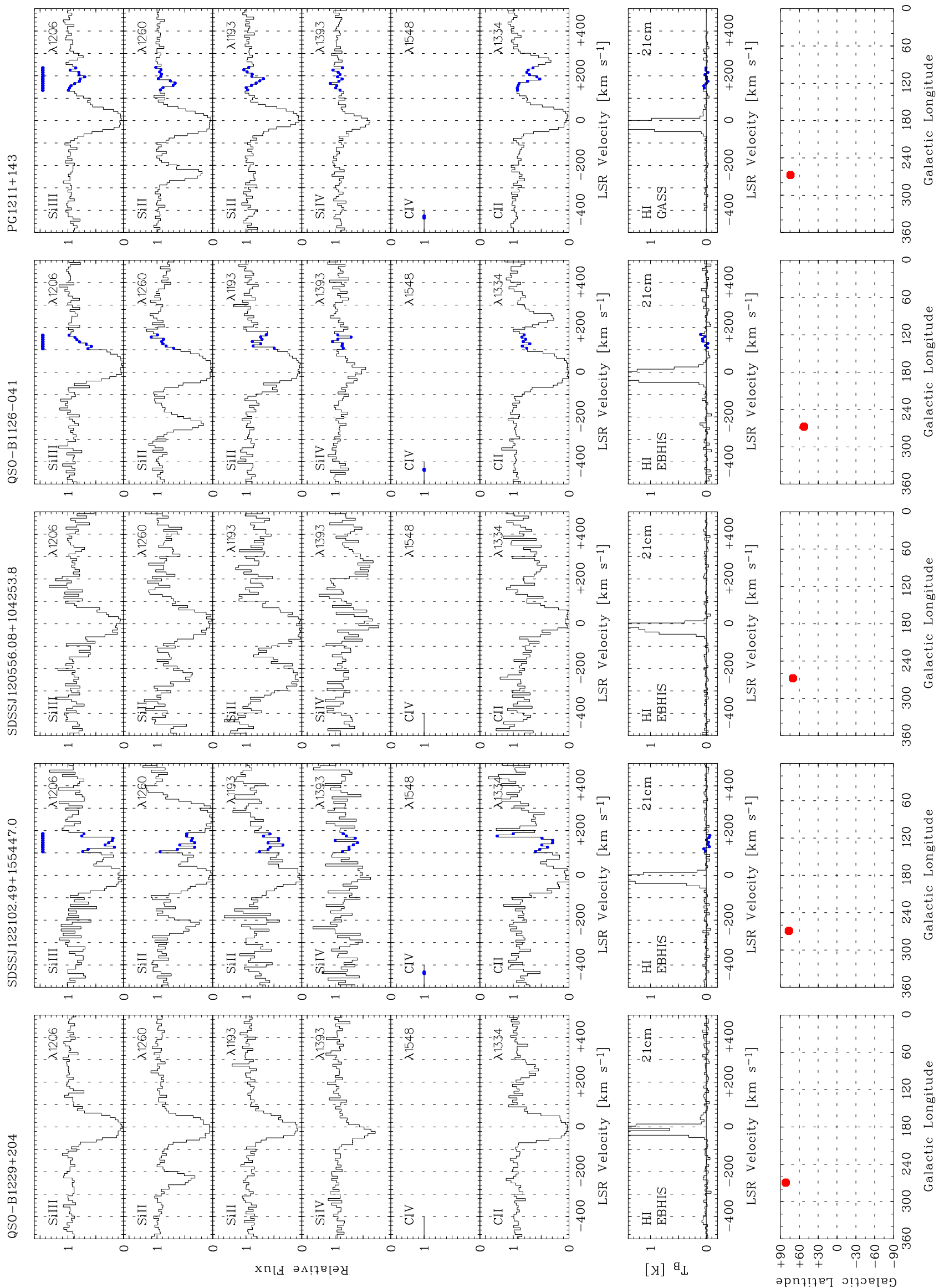

Fig. B.2. continued. 
P. Richter et al.: The Milky Way CGM in absorption
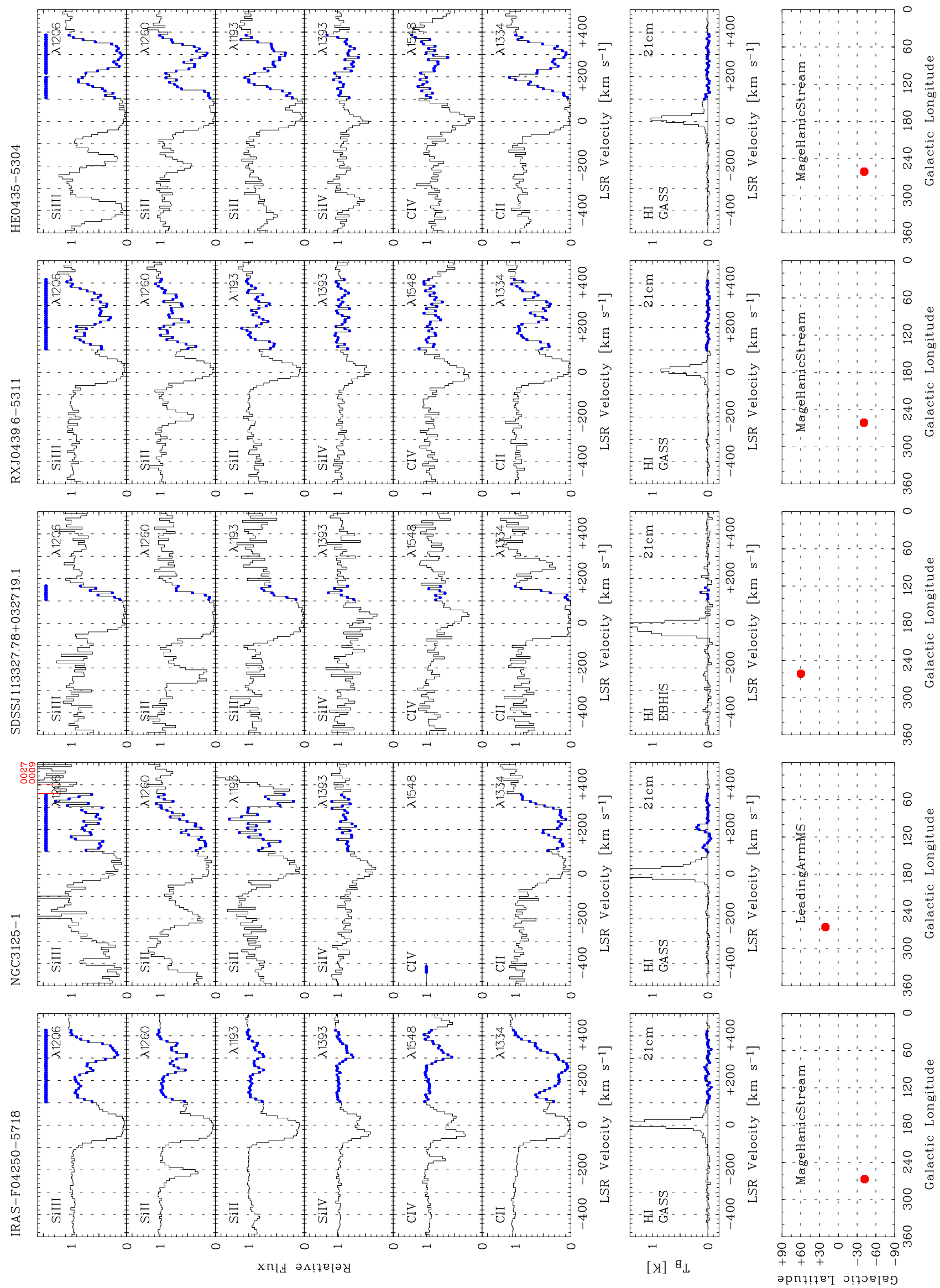

Fig. B.2. continued. 

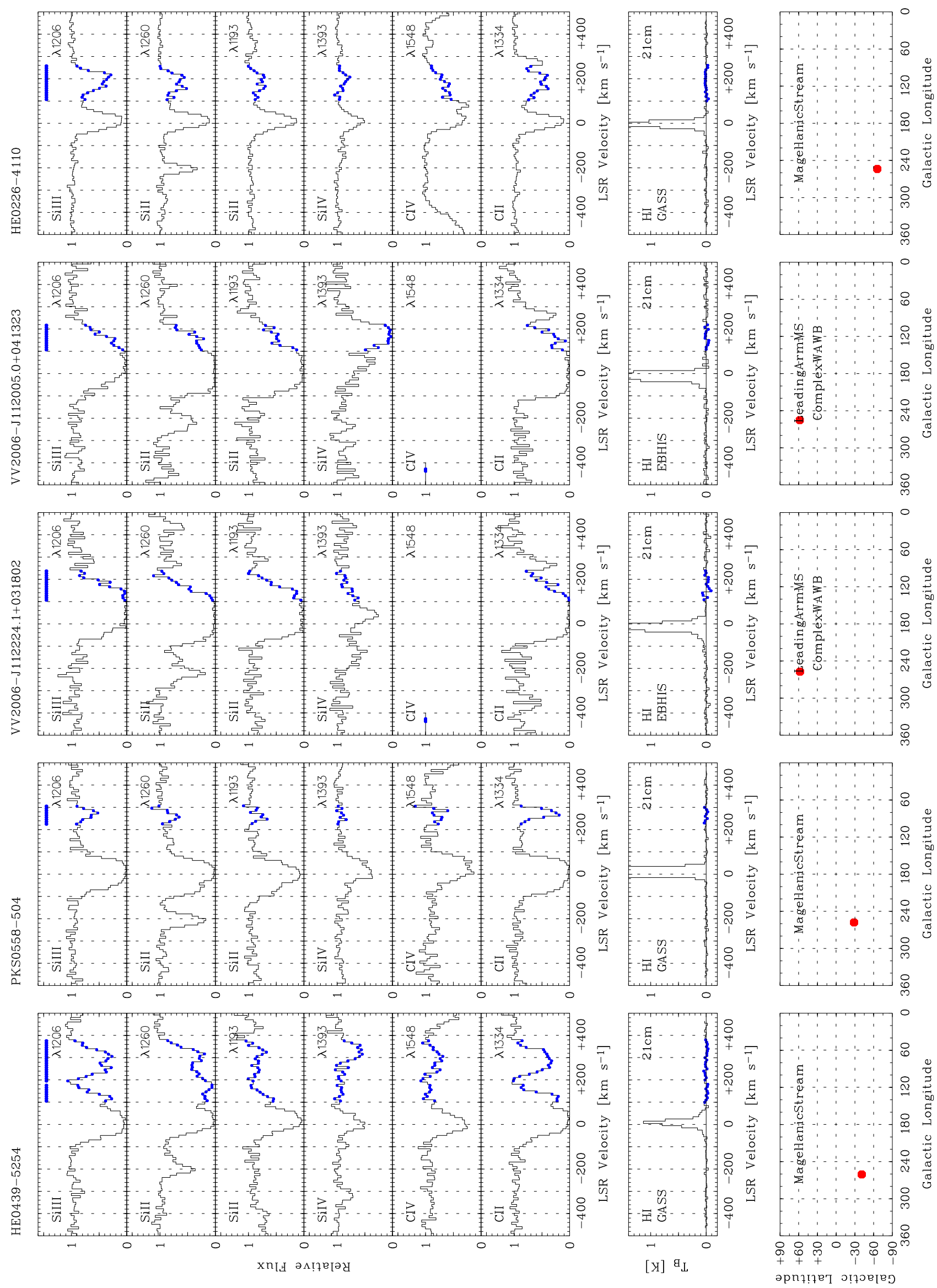

Fig. B.2. continued. 
P. Richter et al.: The Milky Way CGM in absorption
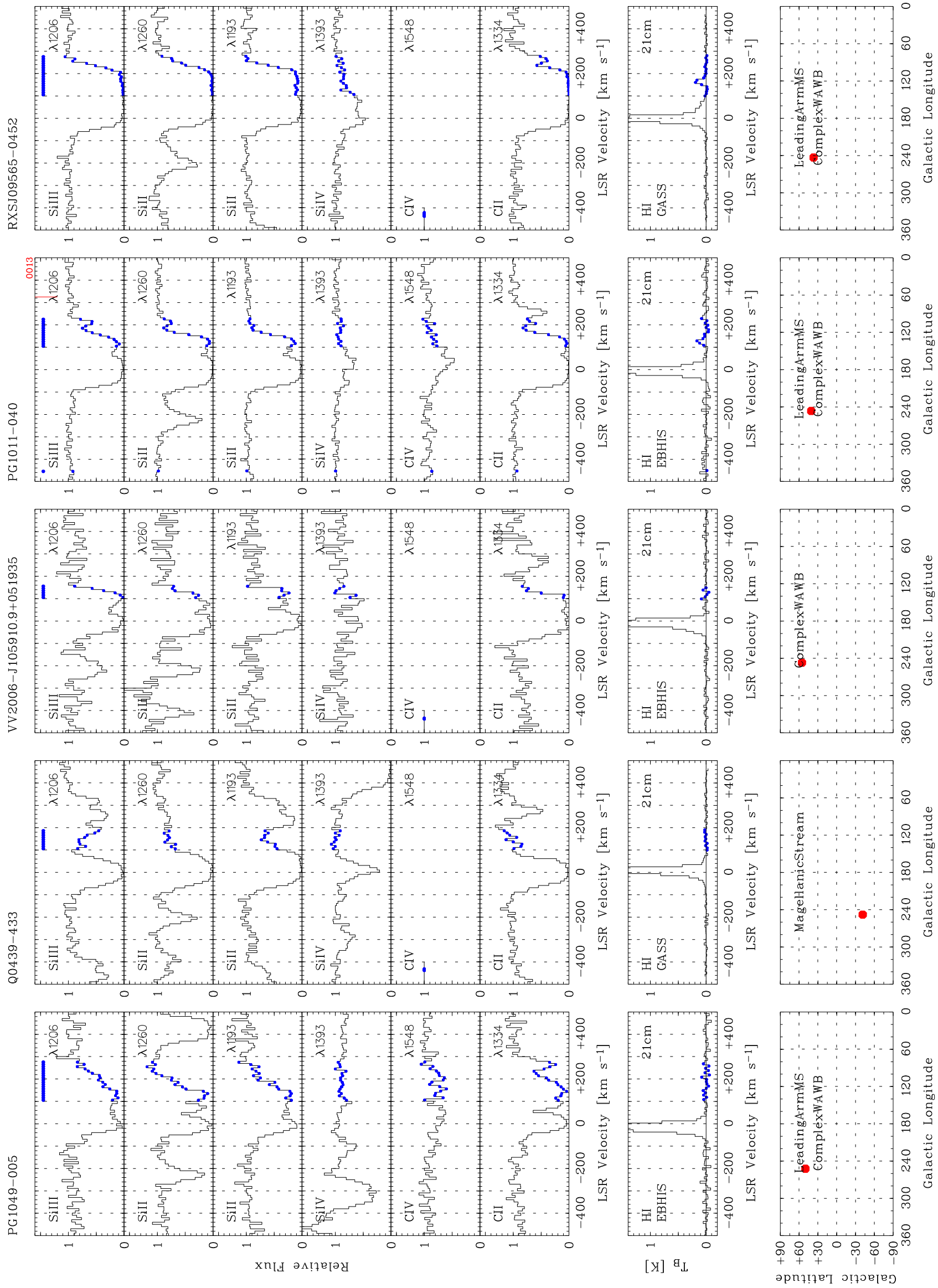

Fig. B.2. continued. 

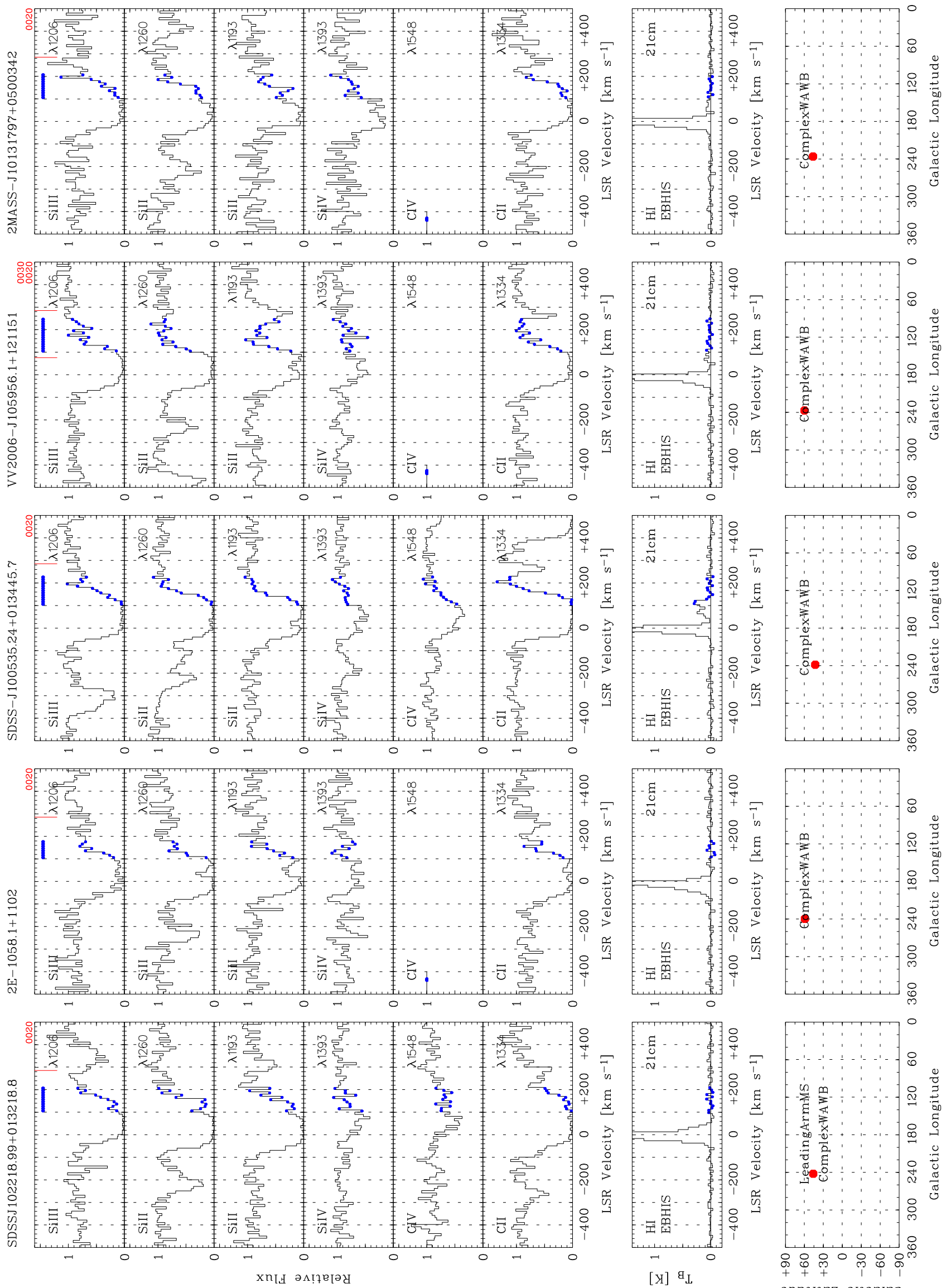

[y] $\mathrm{g}_{\mathrm{L}}$



Fig. B.2. continued. 
P. Richter et al.: The Milky Way CGM in absorption
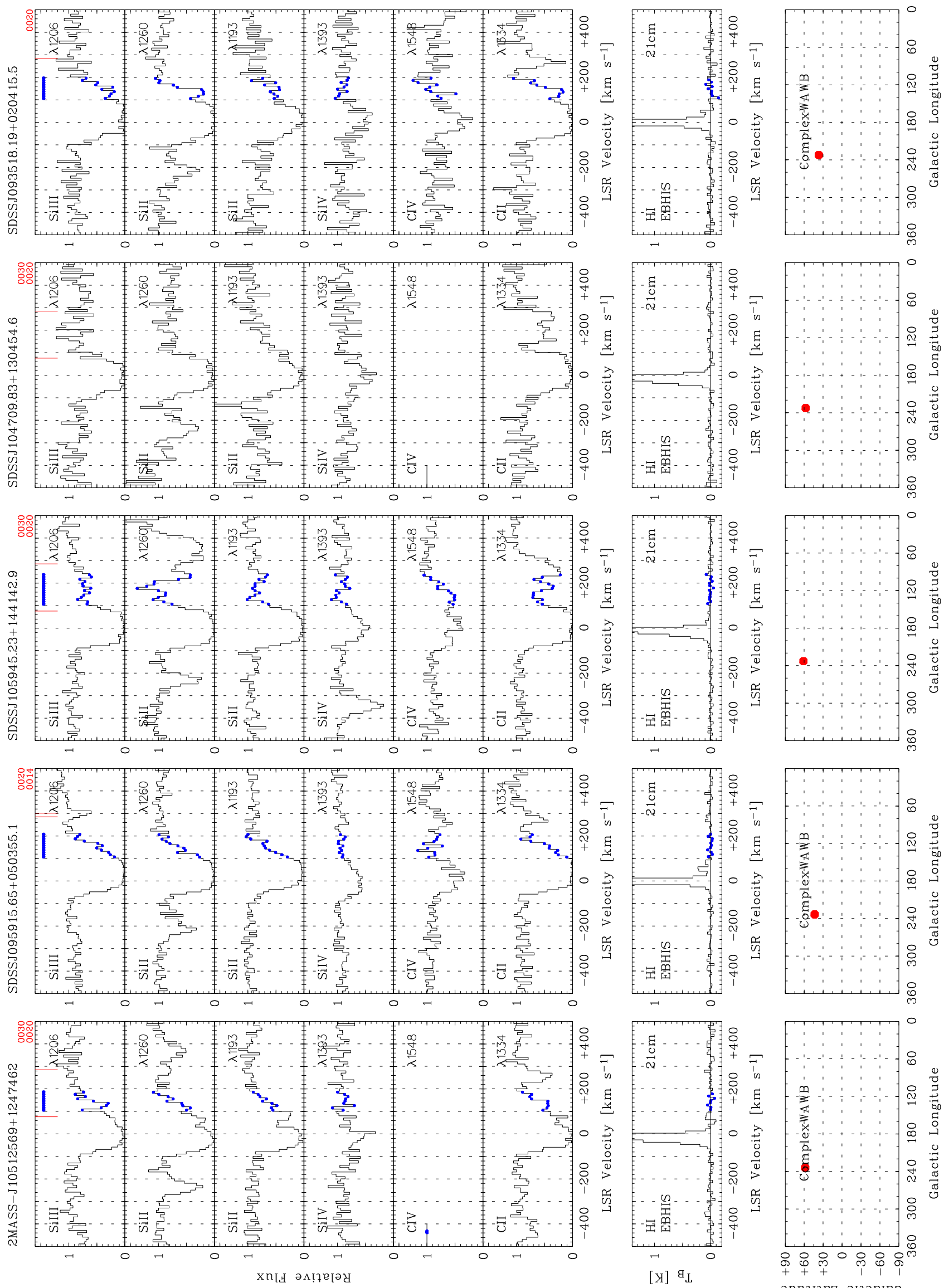

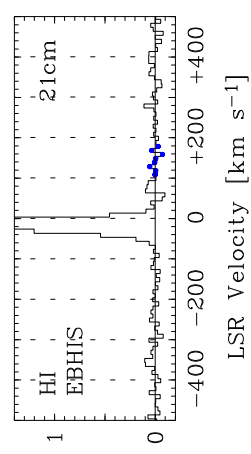

$[\mathrm{Y}] \mathrm{g}_{\mathrm{L}}$

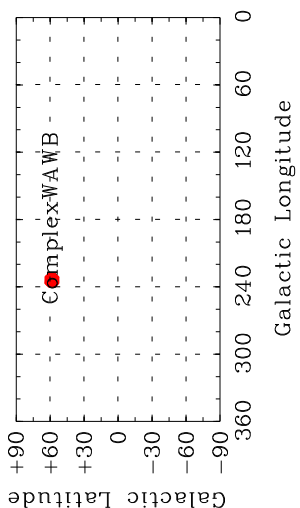

Fig. B.2. continued. 

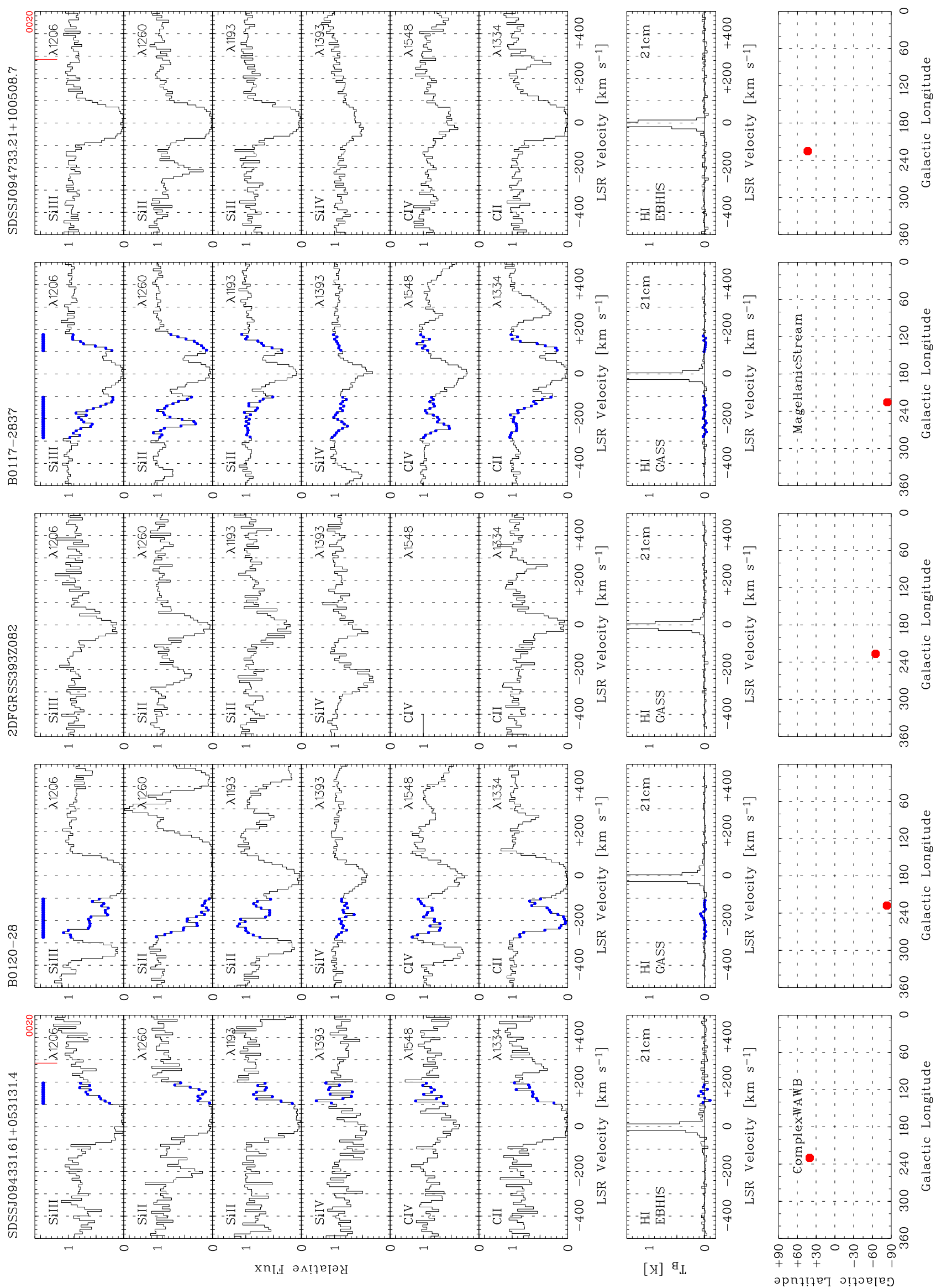

Fig. B.2. continued. 
P. Richter et al.: The Milky Way CGM in absorption
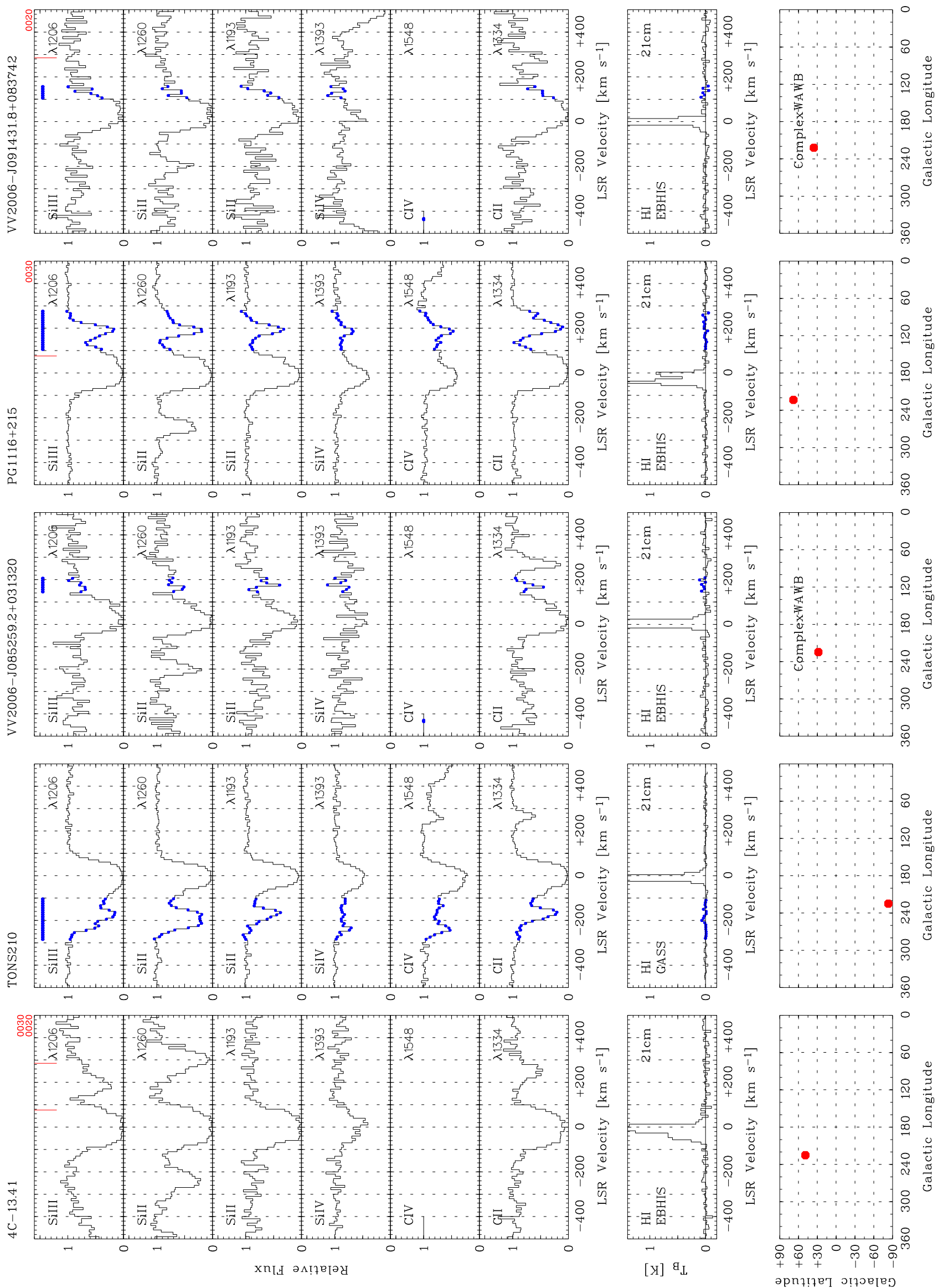

Fig. B.2. continued. 

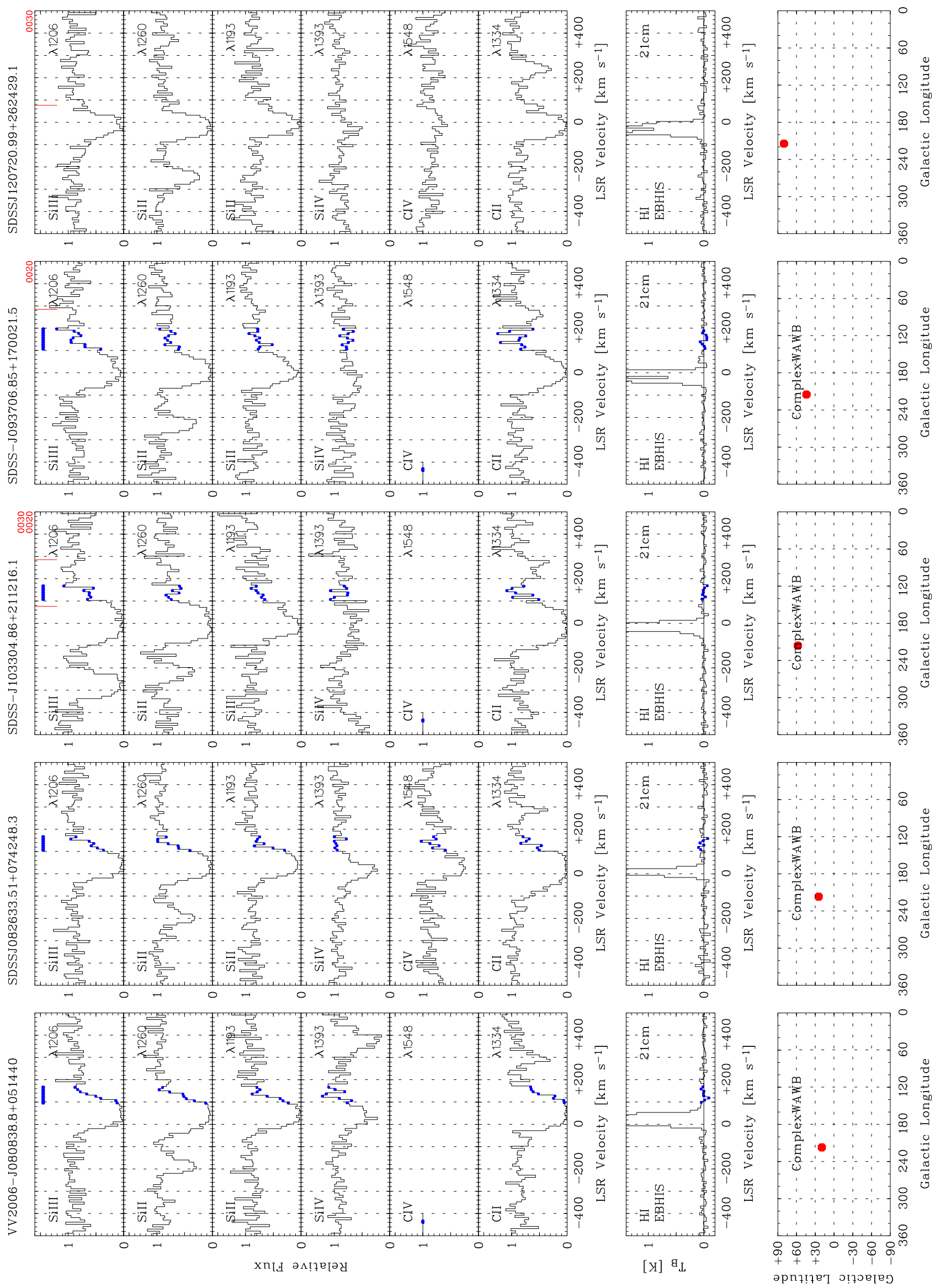

Fig. B.2. continued. 
P. Richter et al.: The Milky Way CGM in absorption
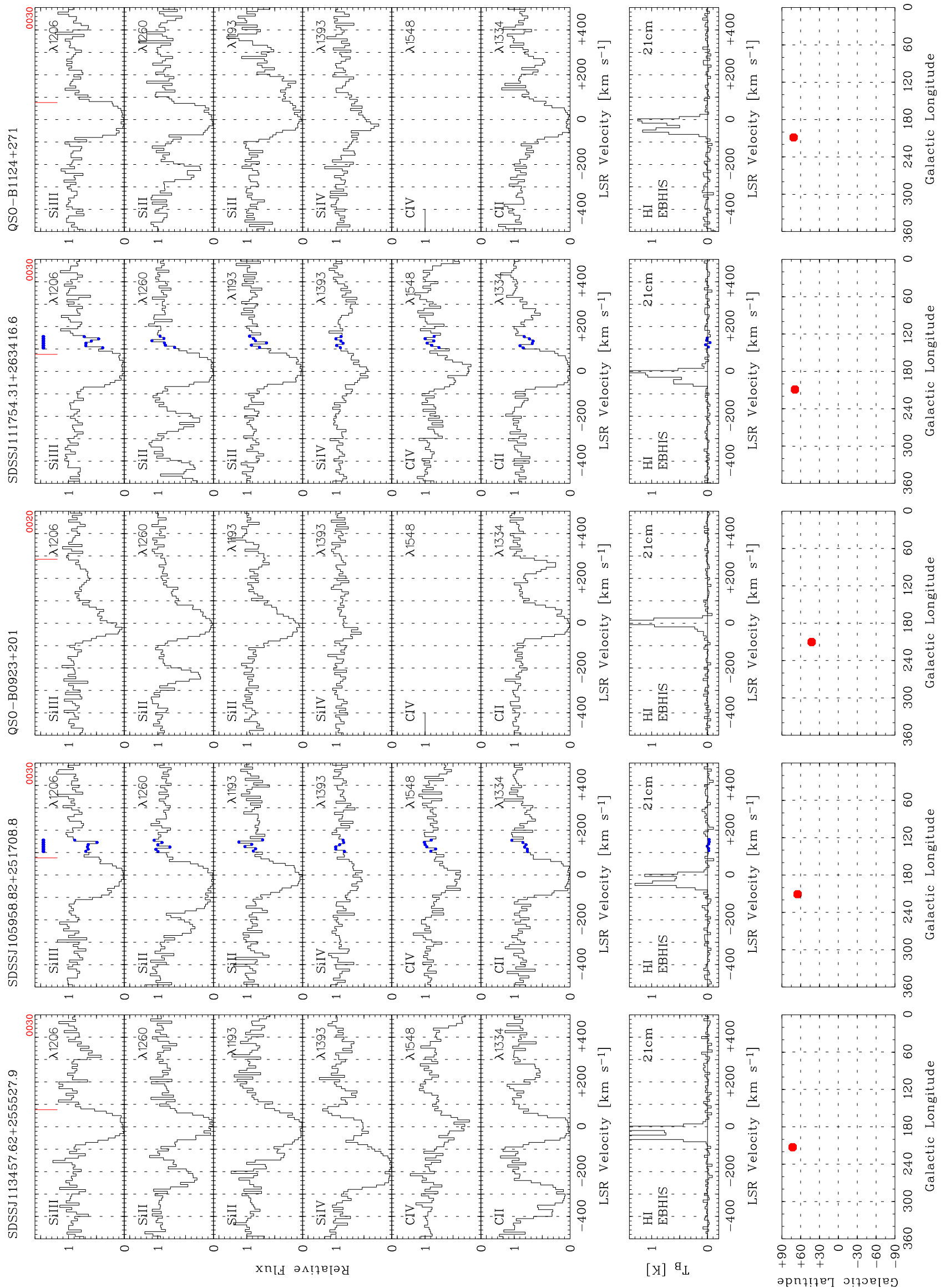

Fig. B.2. continued. 



Fig. B.2. continued. 
P. Richter et al.: The Milky Way CGM in absorption


Fig. B.2. continued. 

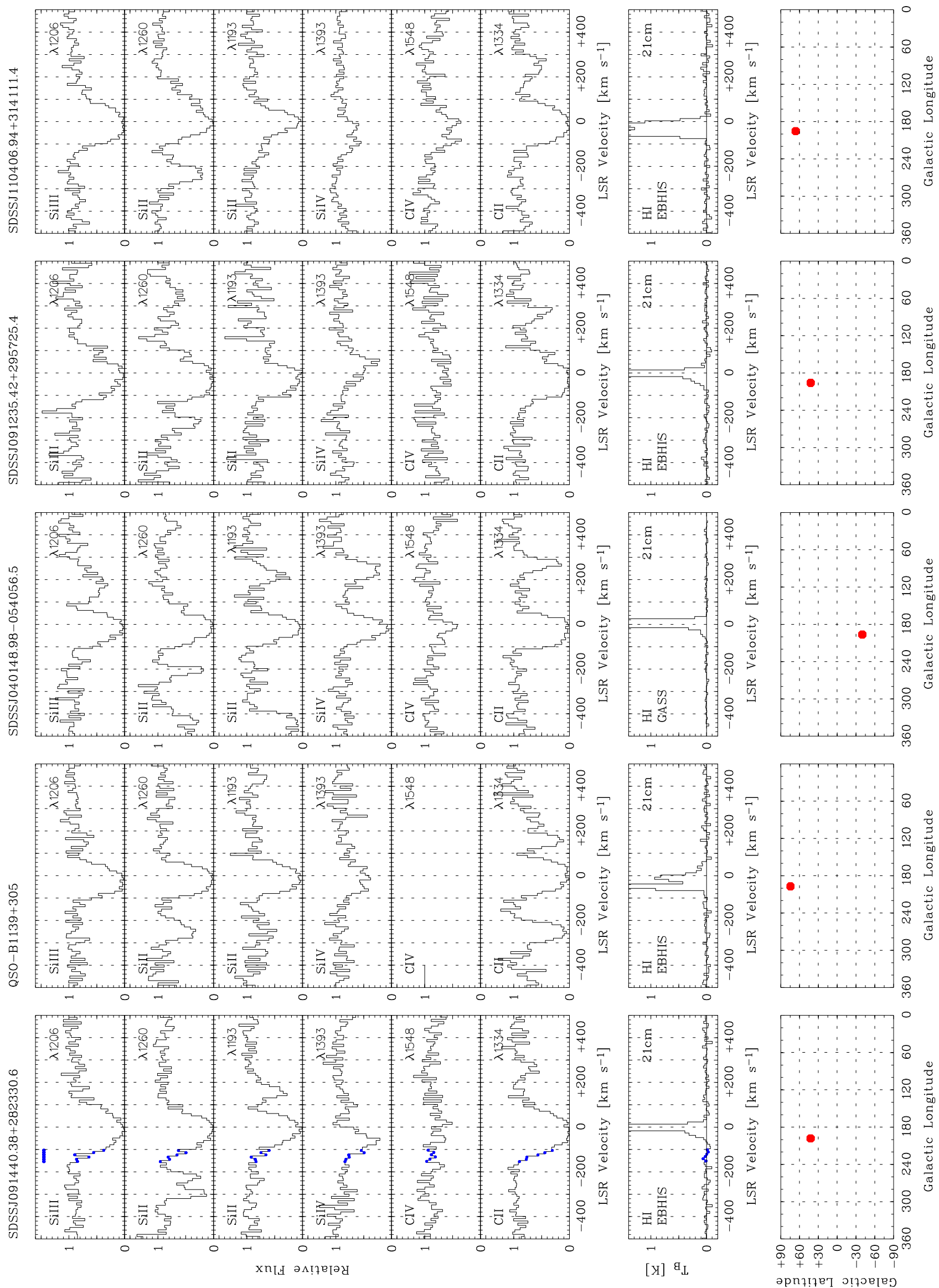

Fig. B.2. continued. 
P. Richter et al.: The Milky Way CGM in absorption
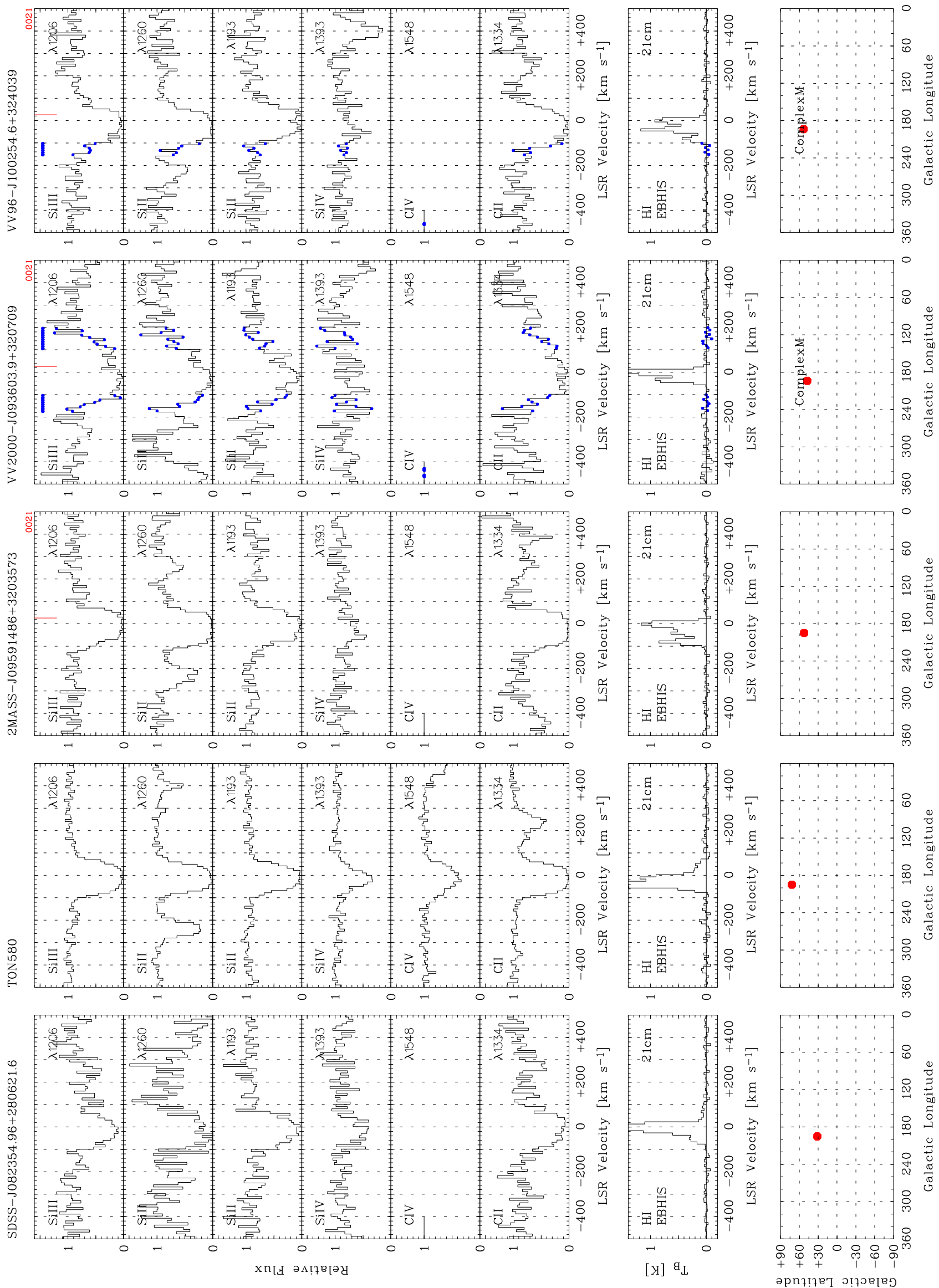

Fig. B.2. continued. 



Fig. B.2. continued. 
P. Richter et al.: The Milky Way CGM in absorption
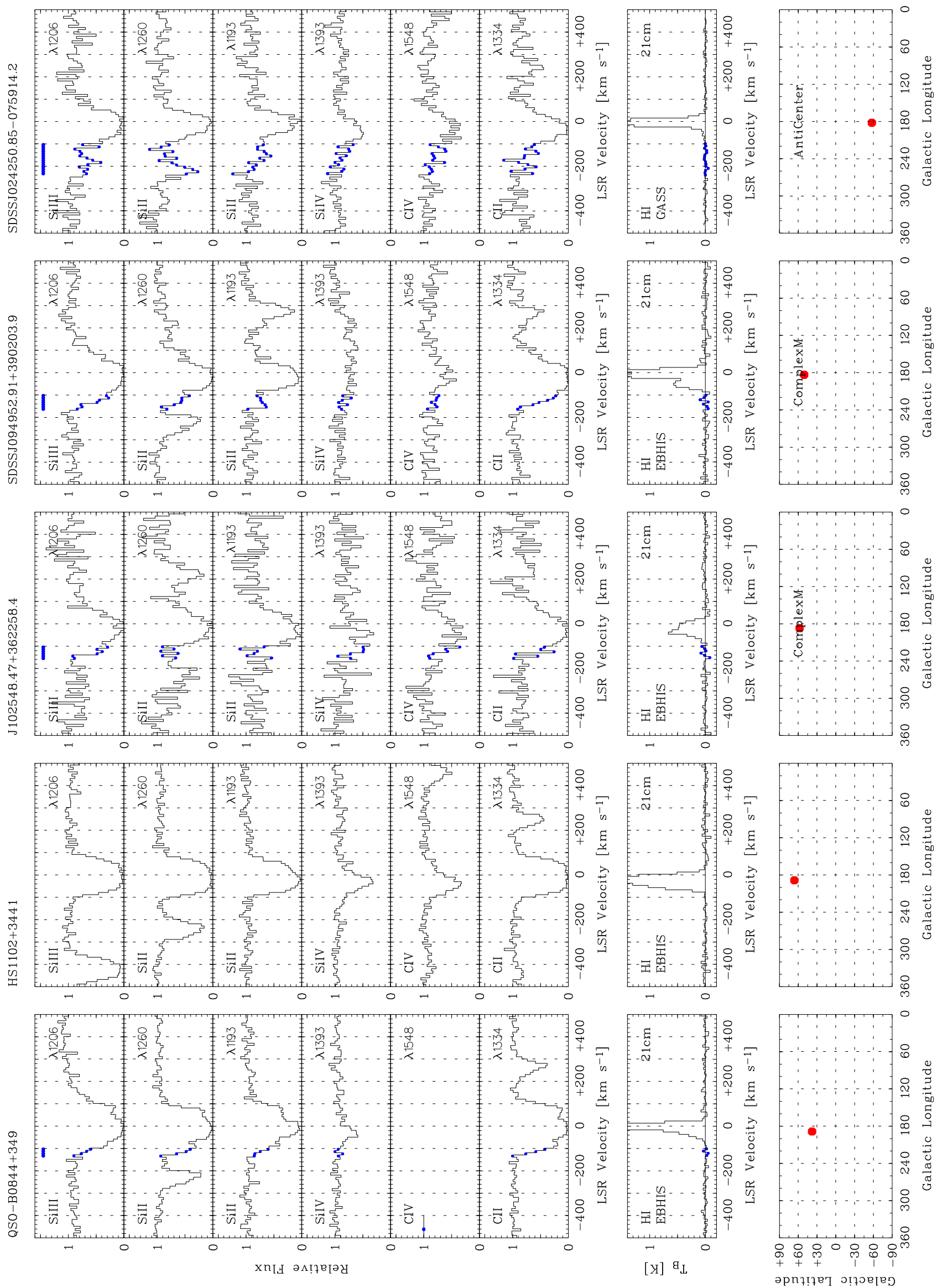

Fig. B.2. continued. 

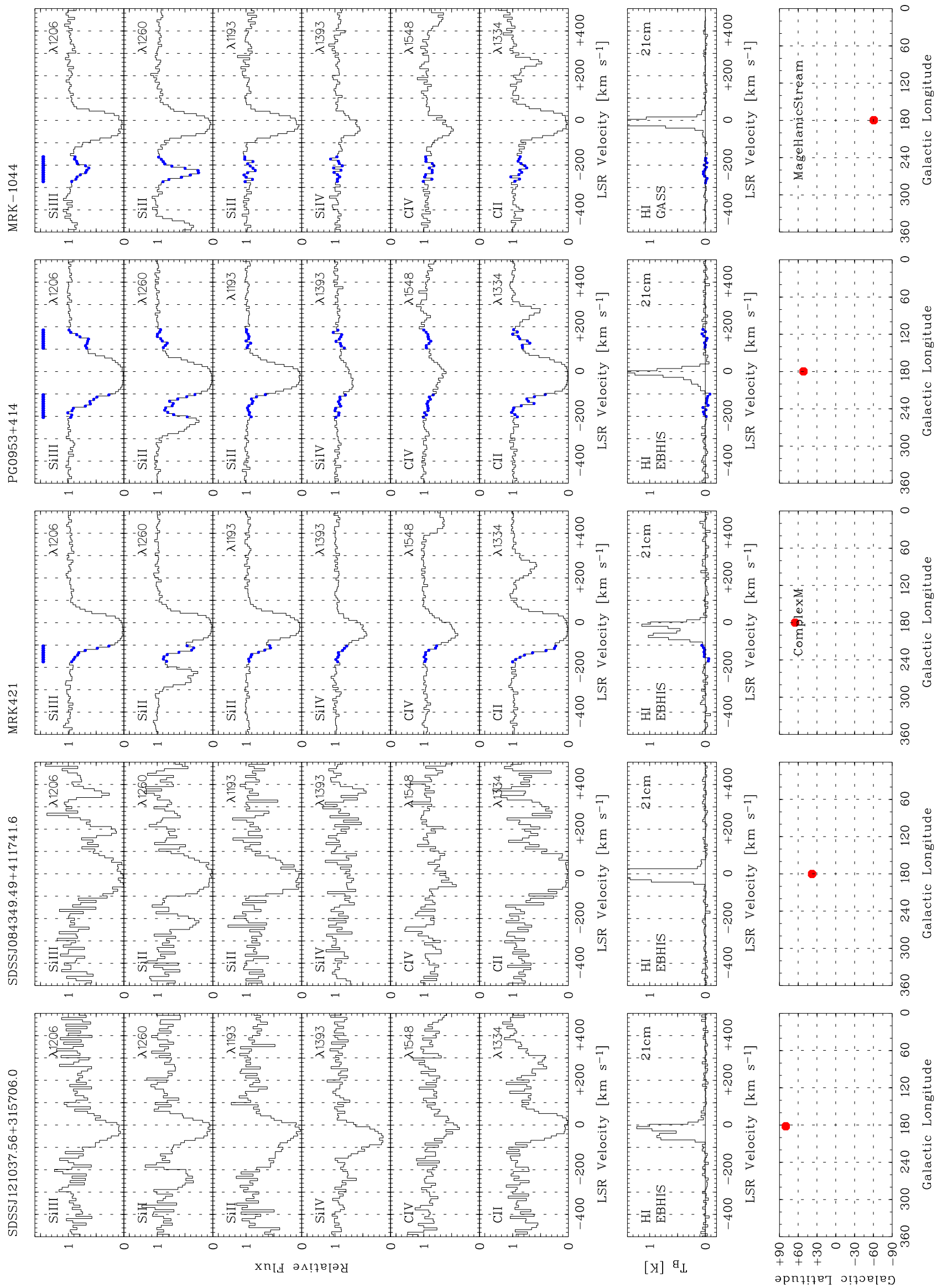

Fig. B.2. continued. 
P. Richter et al.: The Milky Way CGM in absorption
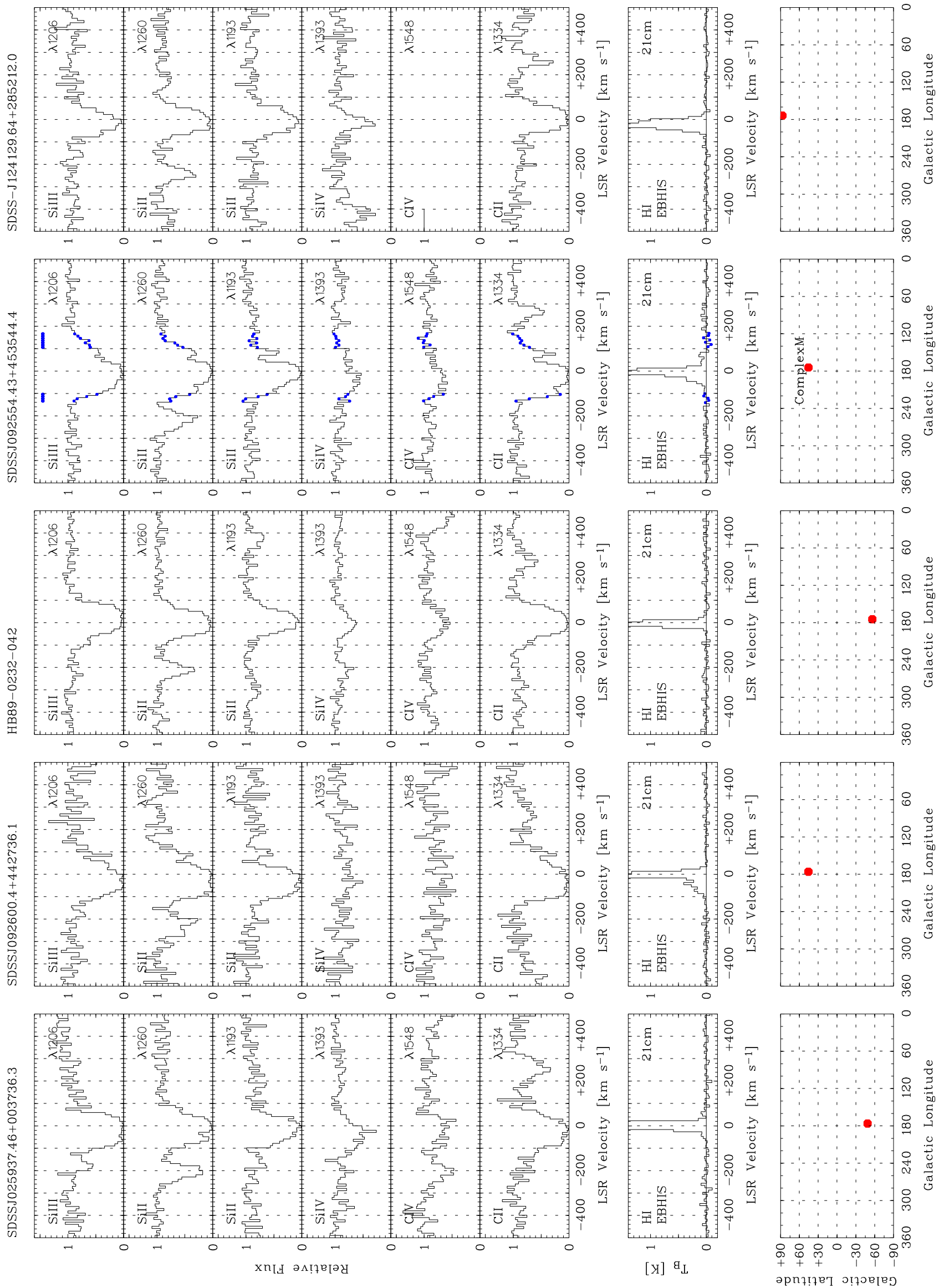

Fig. B.2. continued. 

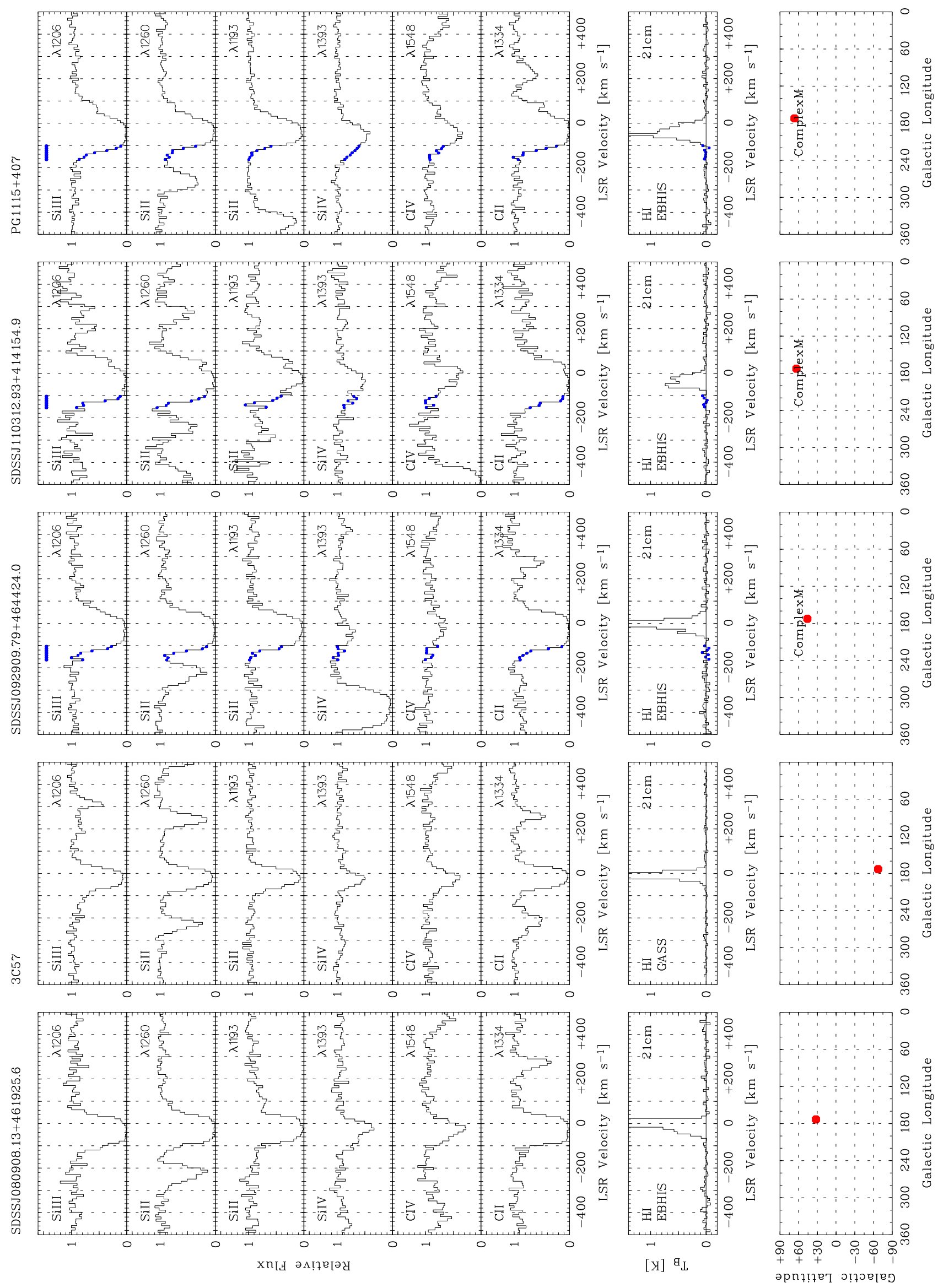

Fig. B.2. continued. 
P. Richter et al.: The Milky Way CGM in absorption
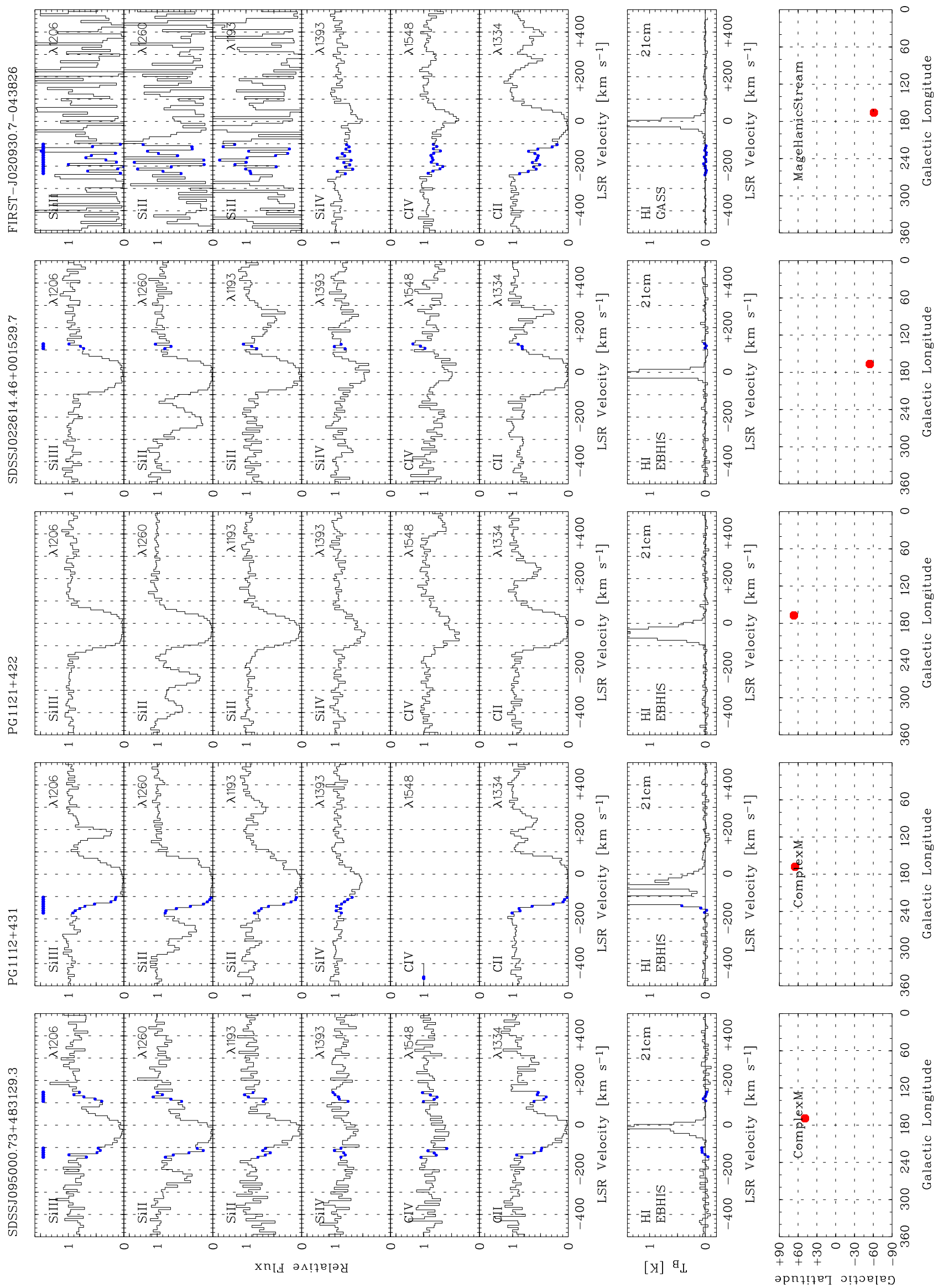

Fig. B.2. continued. 

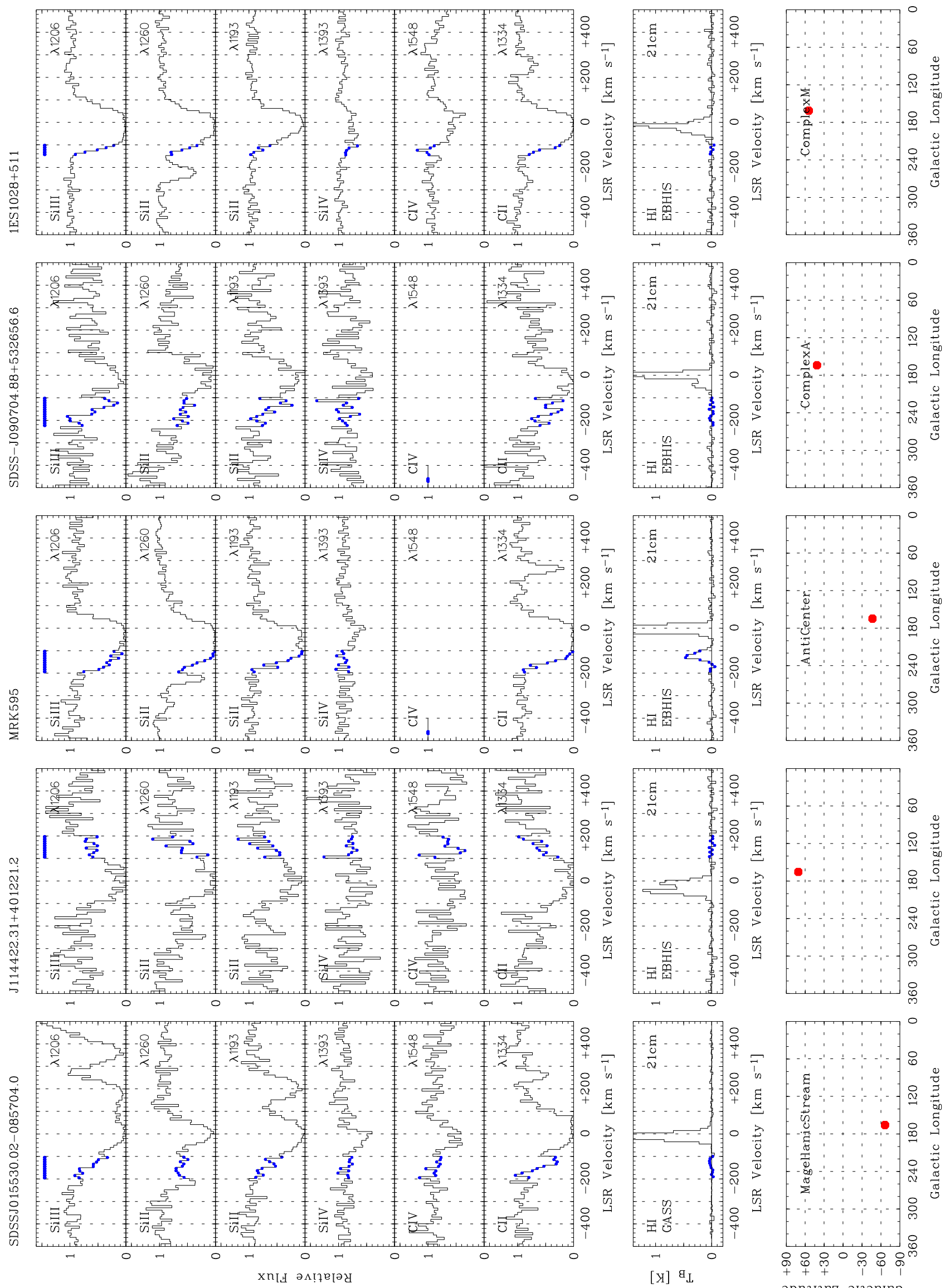

Fig. B.2. continued. 
P. Richter et al.: The Milky Way CGM in absorption
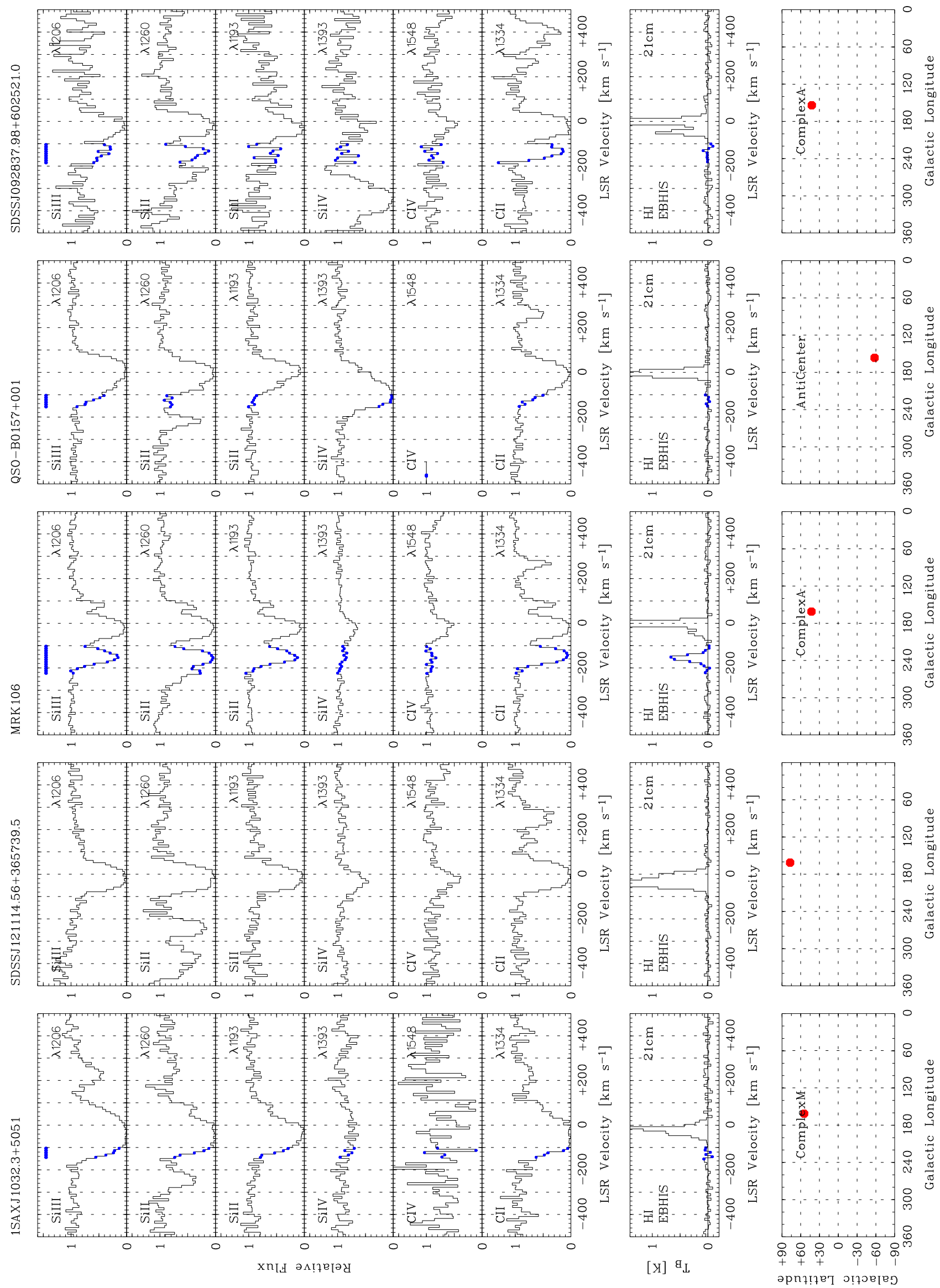

Fig. B.2. continued. 

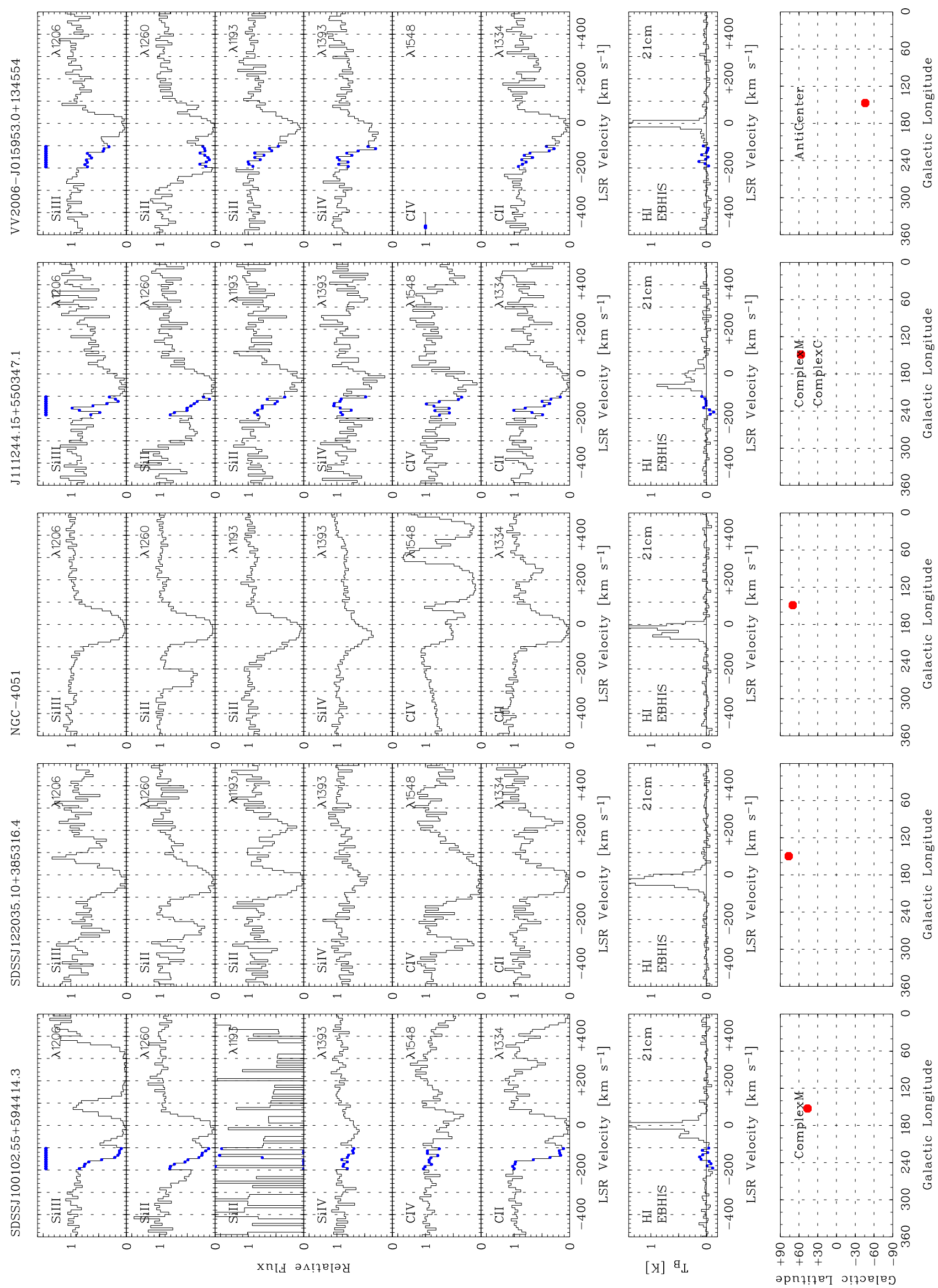

Fig. B.2. continued. 
P. Richter et al.: The Milky Way CGM in absorption
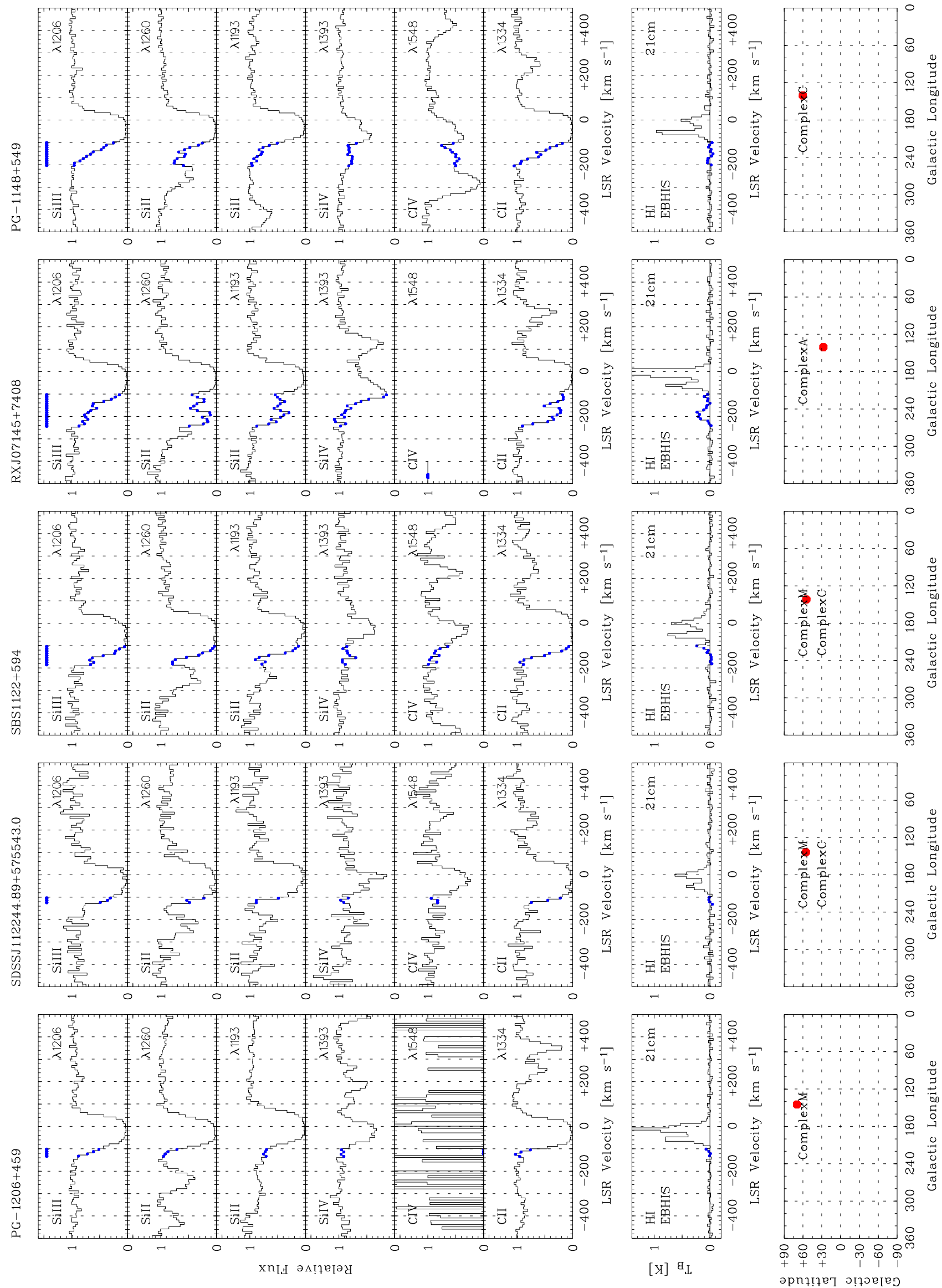

Fig. B.2. continued. 

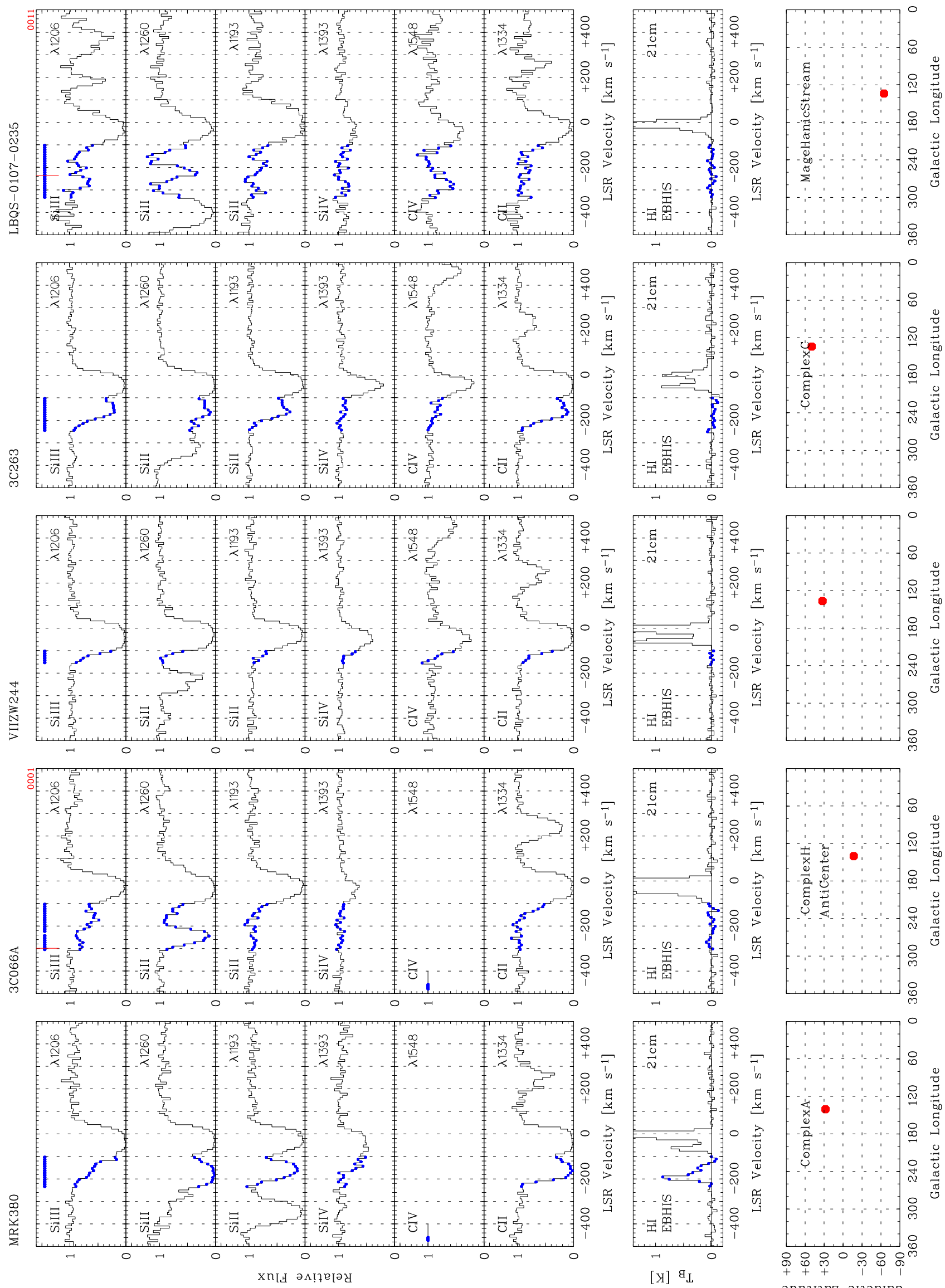

Fig. B.2. continued. 
P. Richter et al.: The Milky Way CGM in absorption
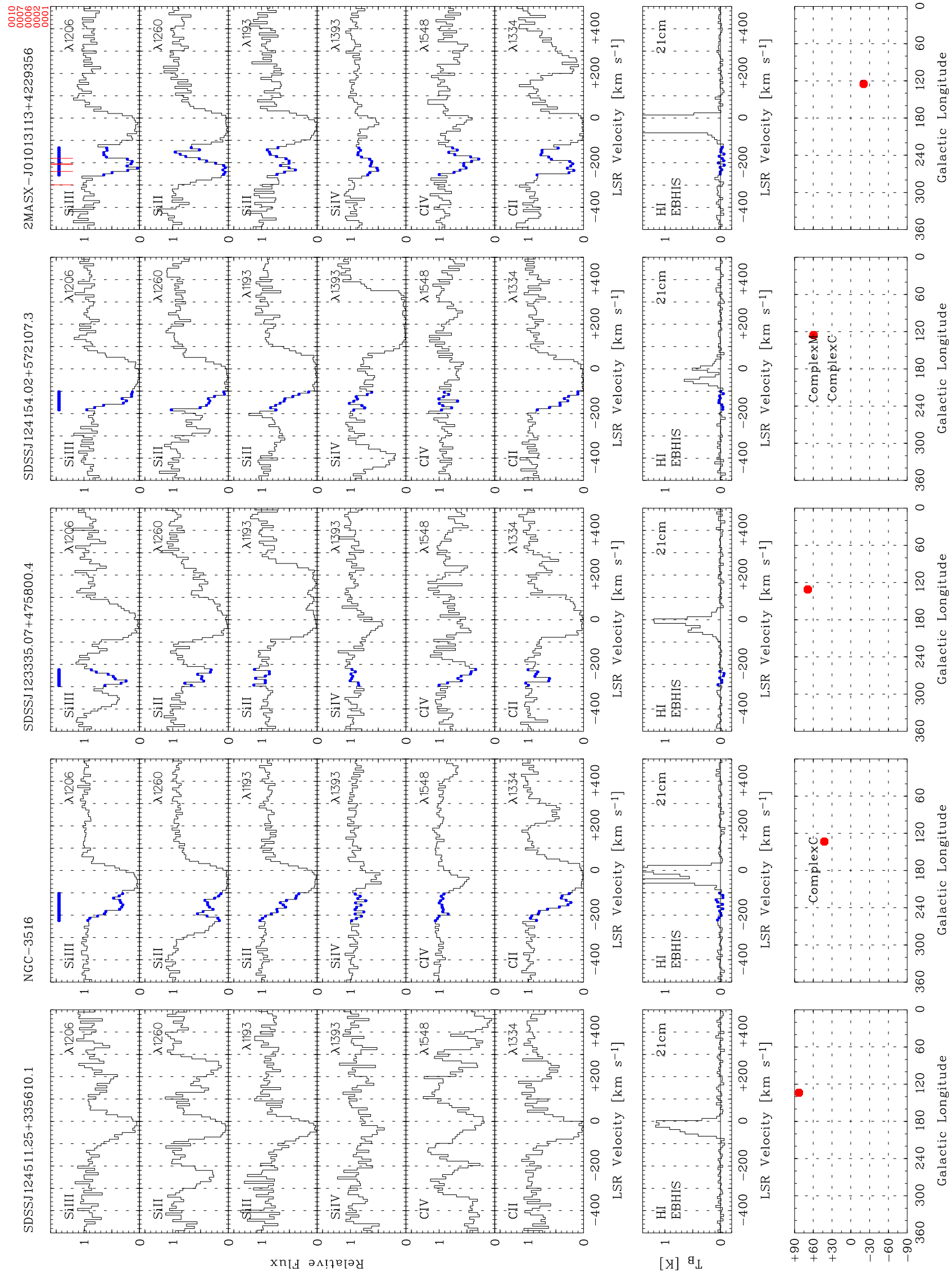

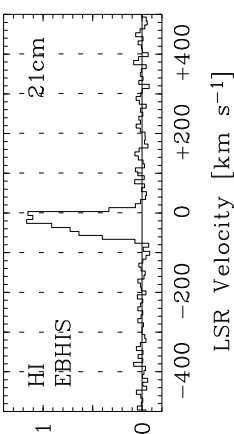

[y] ${ }_{\mathrm{L}}$

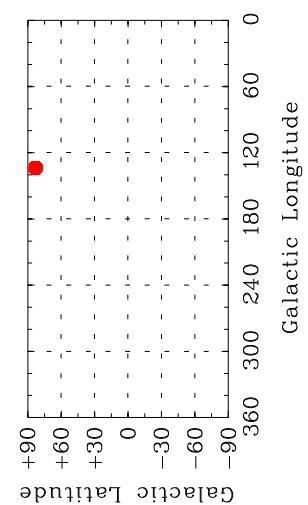

Fig. B.2. continued. 

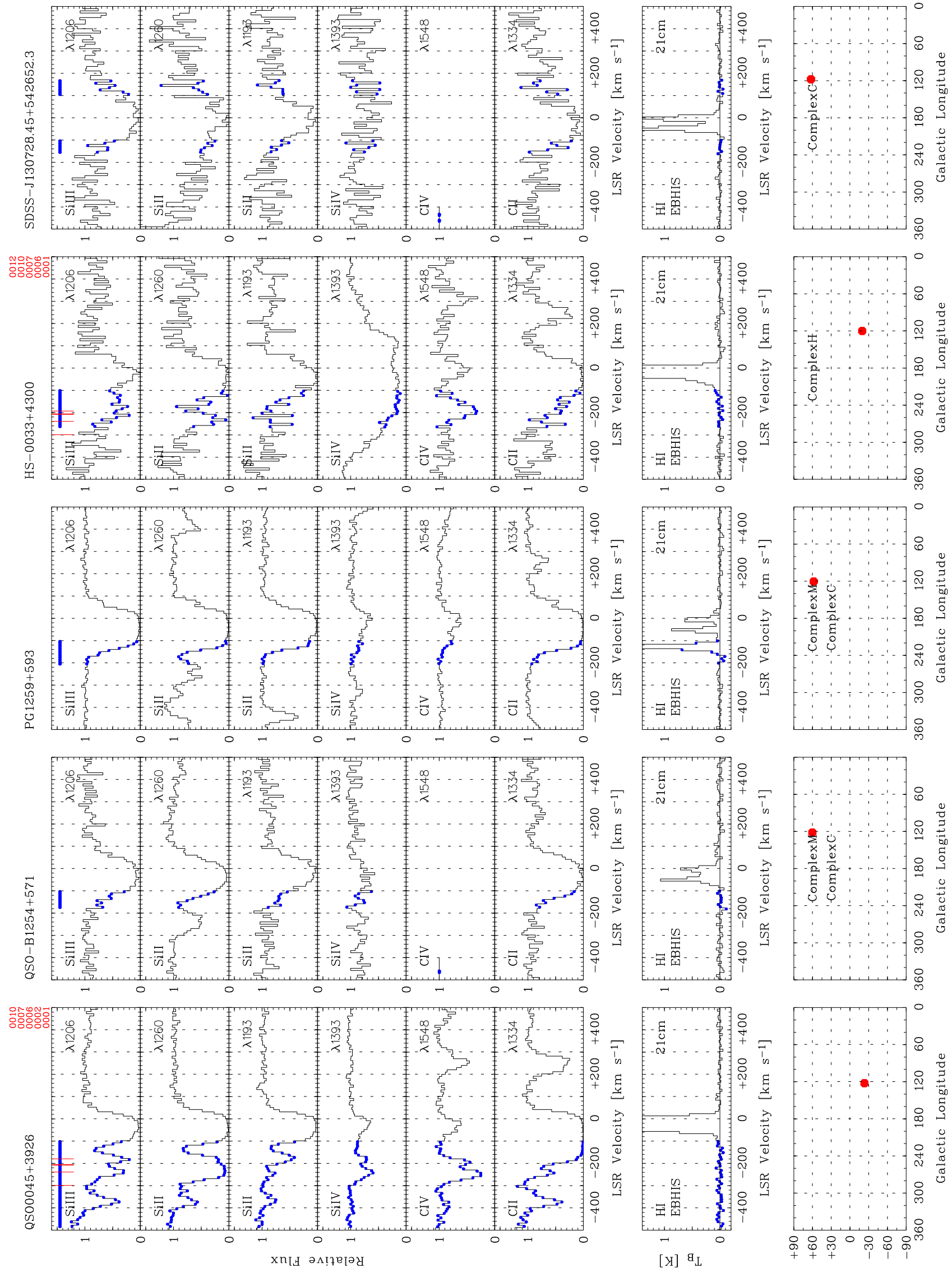

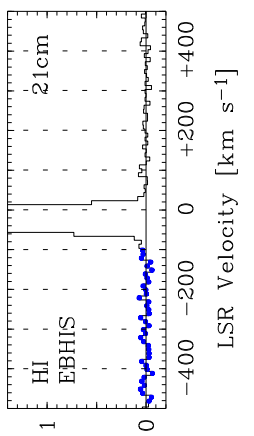

[y] ${ }^{\mathrm{L}}$

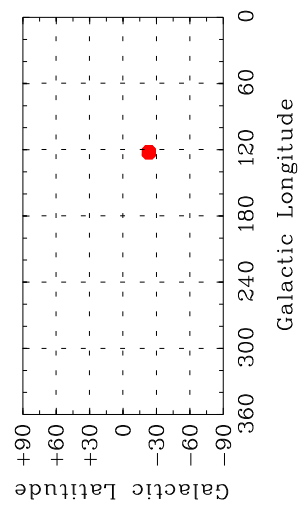

Fig. B.2. continued. 
P. Richter et al.: The Milky Way CGM in absorption
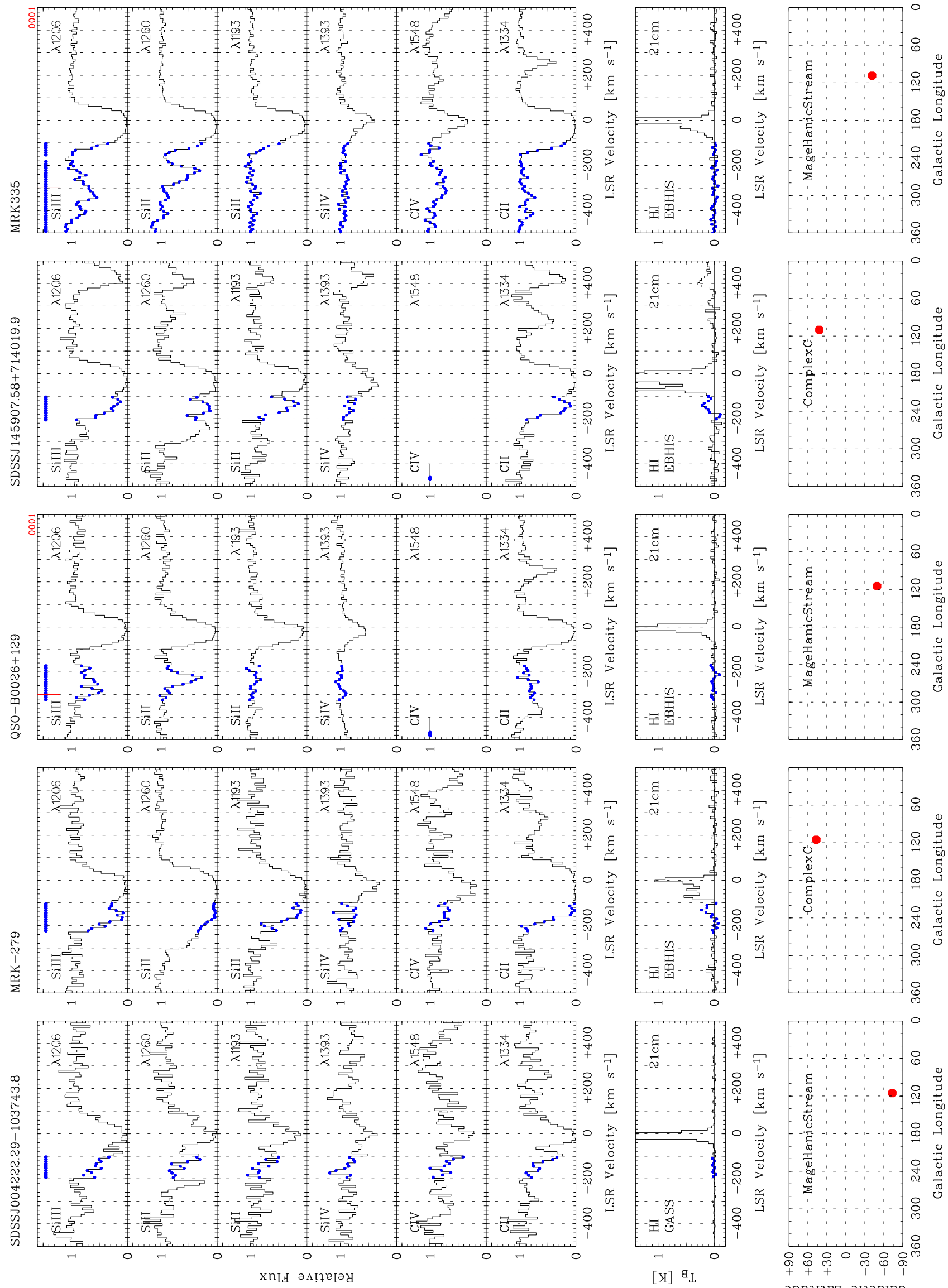

Fig. B.2. continued. 

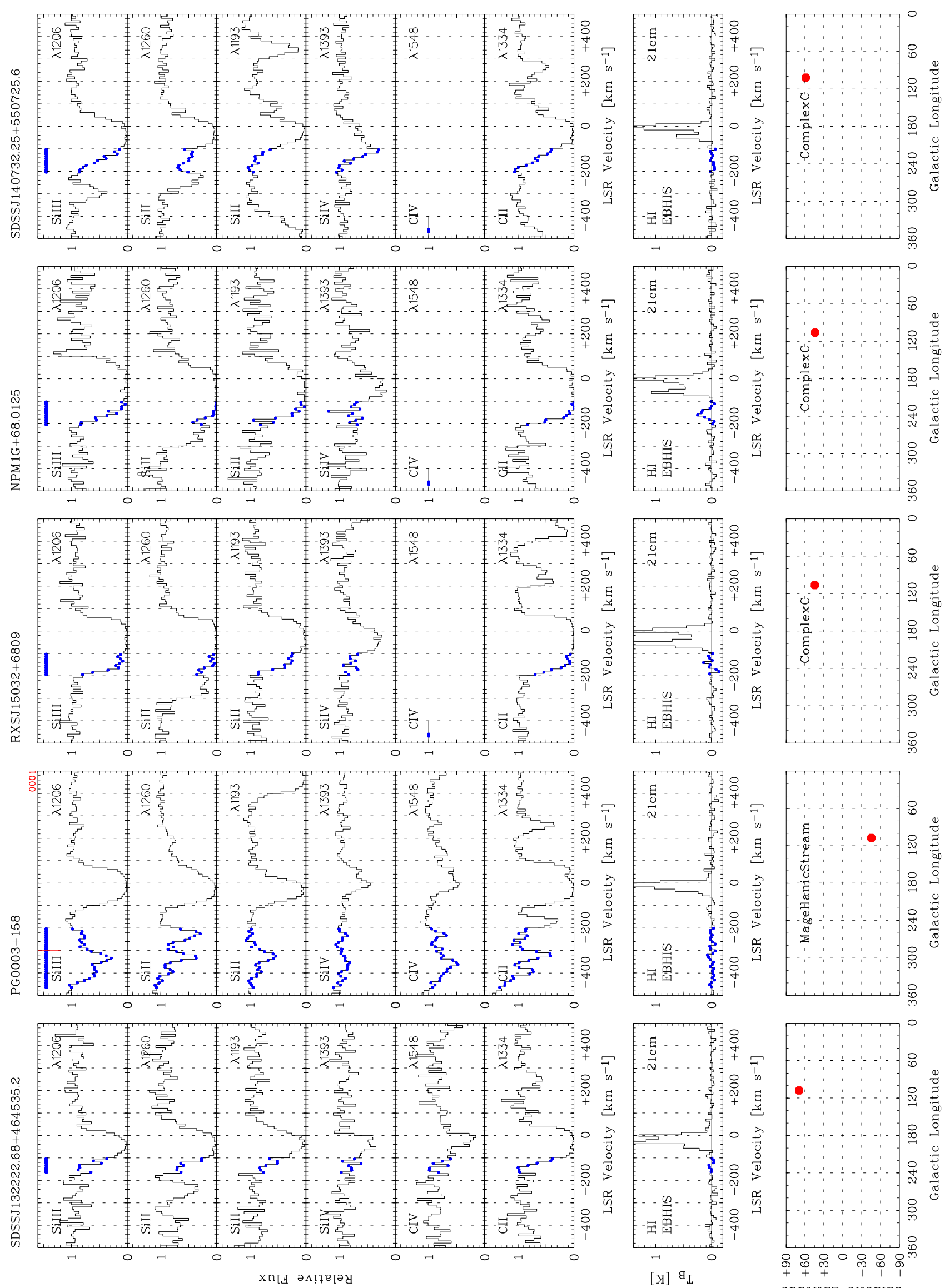

Fig. B.2. continued. 
P. Richter et al.: The Milky Way CGM in absorption
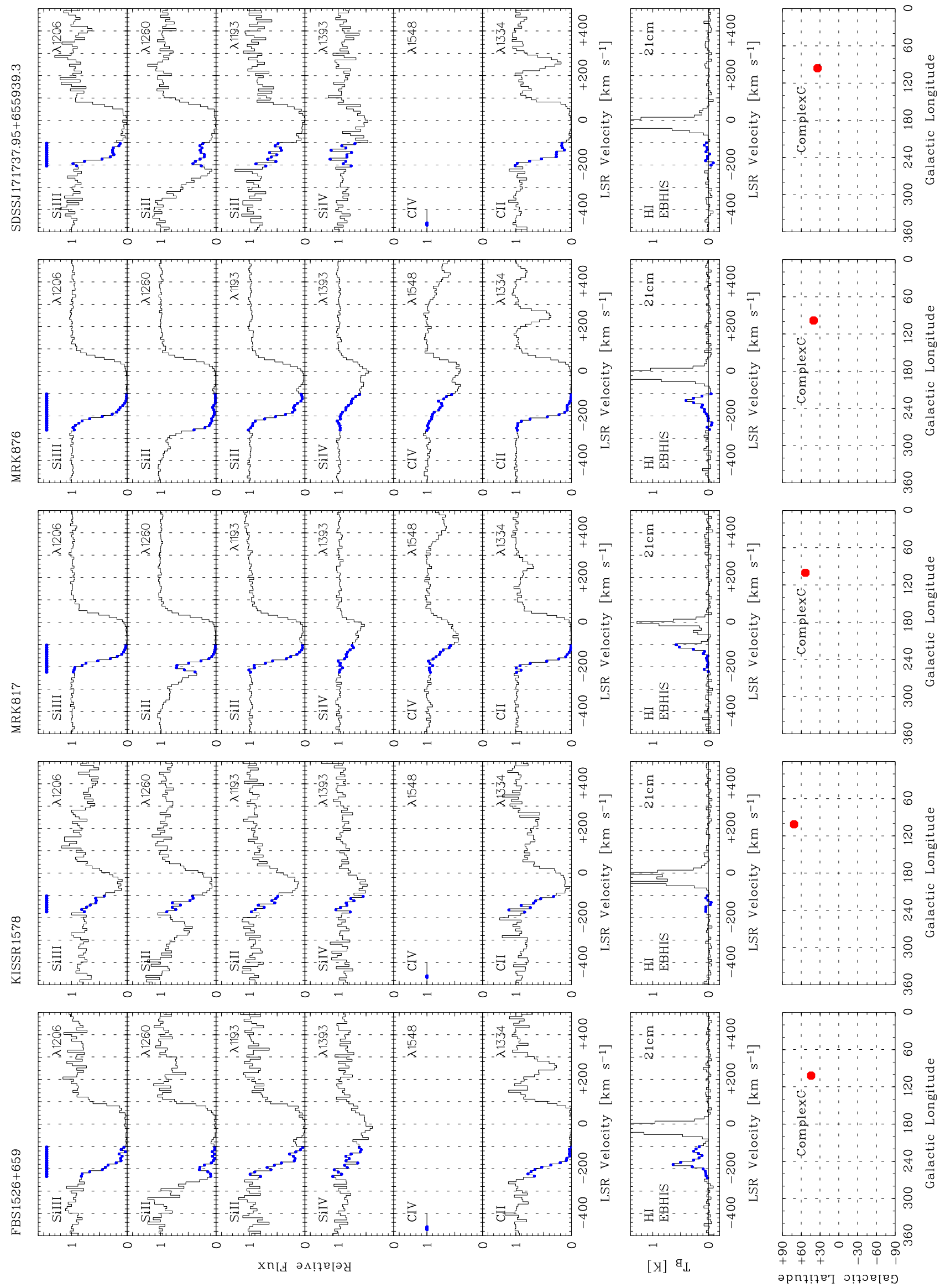

Fig. B.2. continued. 

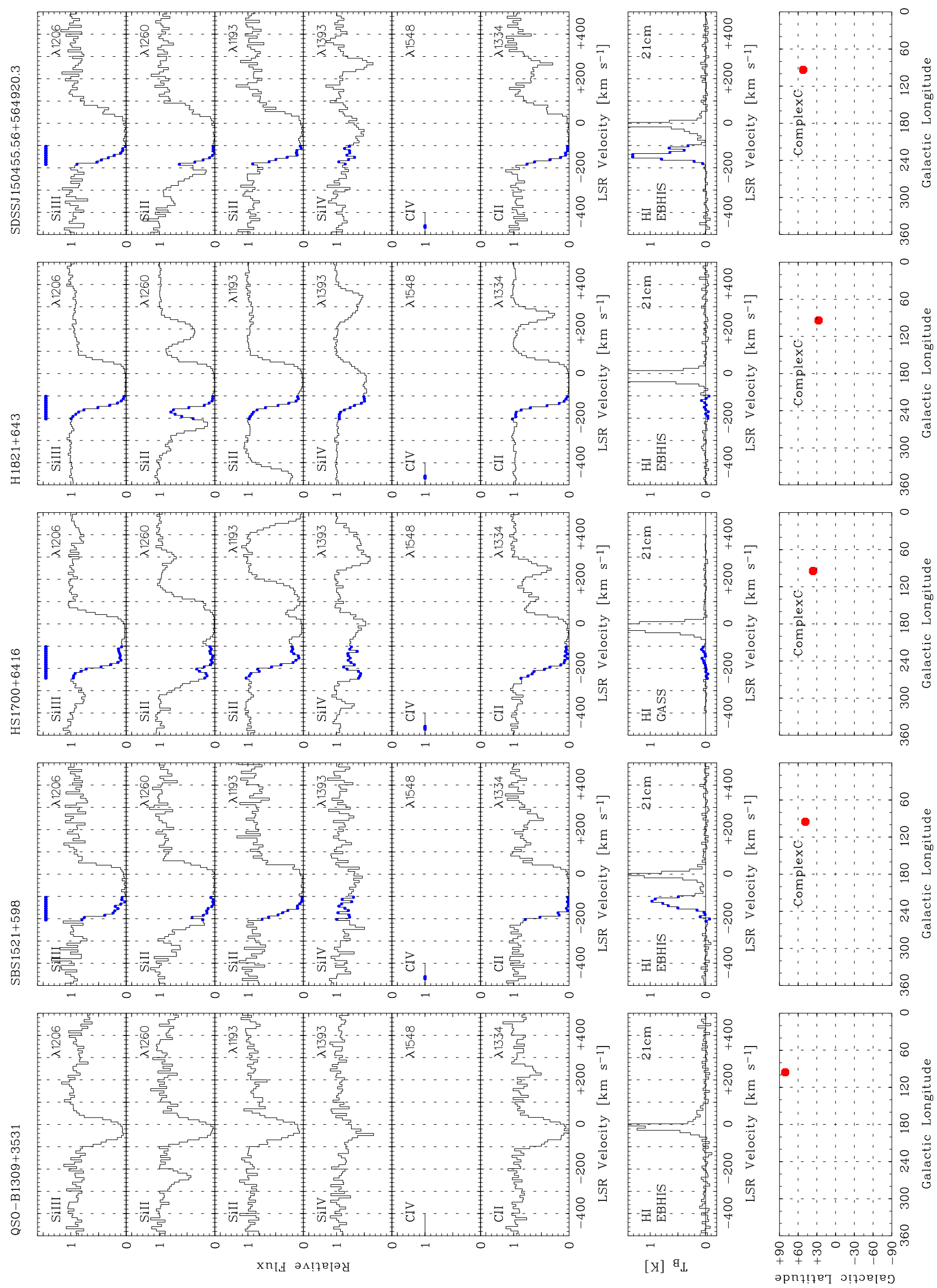

Fig. B.2. continued. 
P. Richter et al.: The Milky Way CGM in absorption
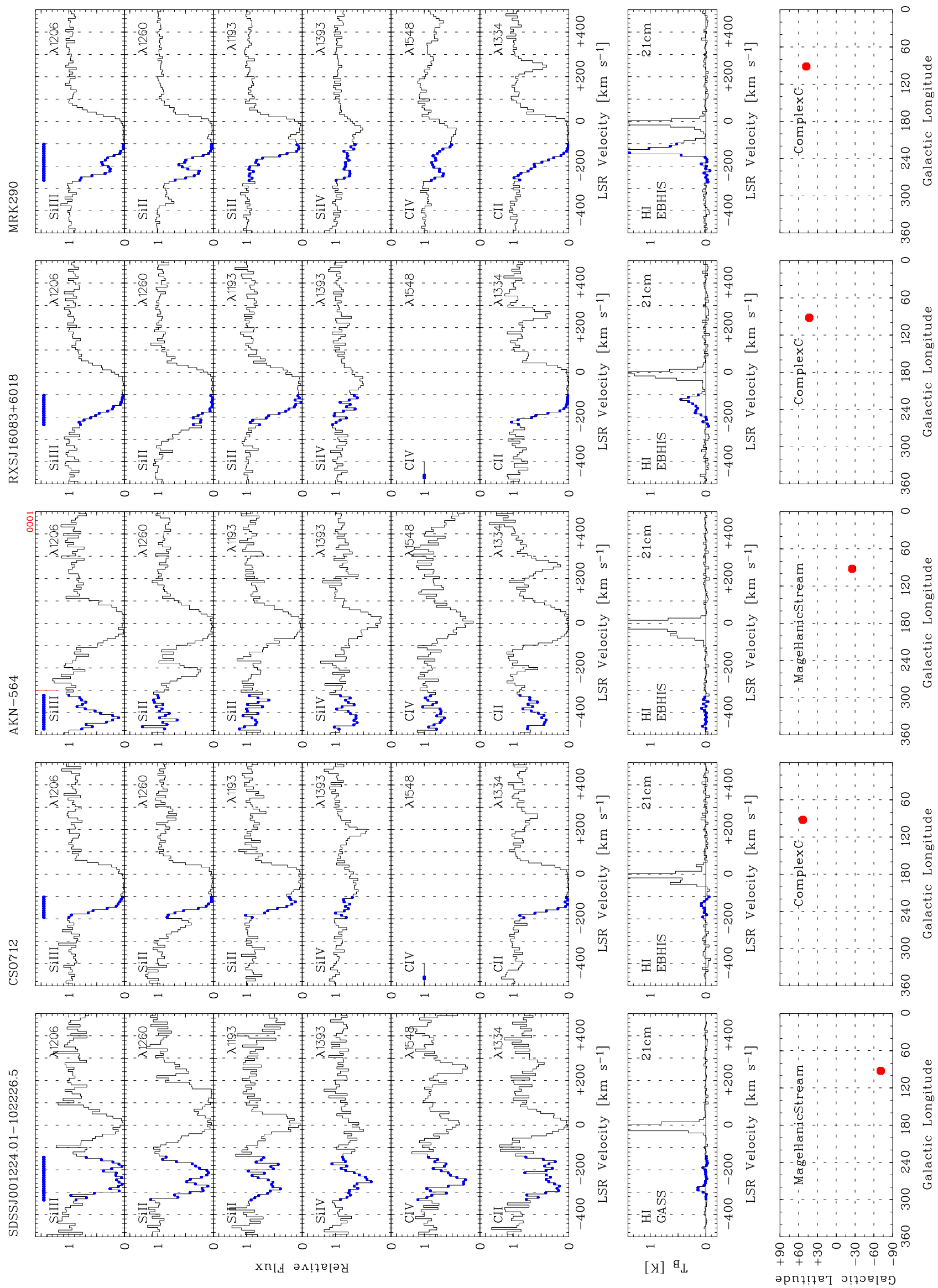

Fig. B.2. continued. 

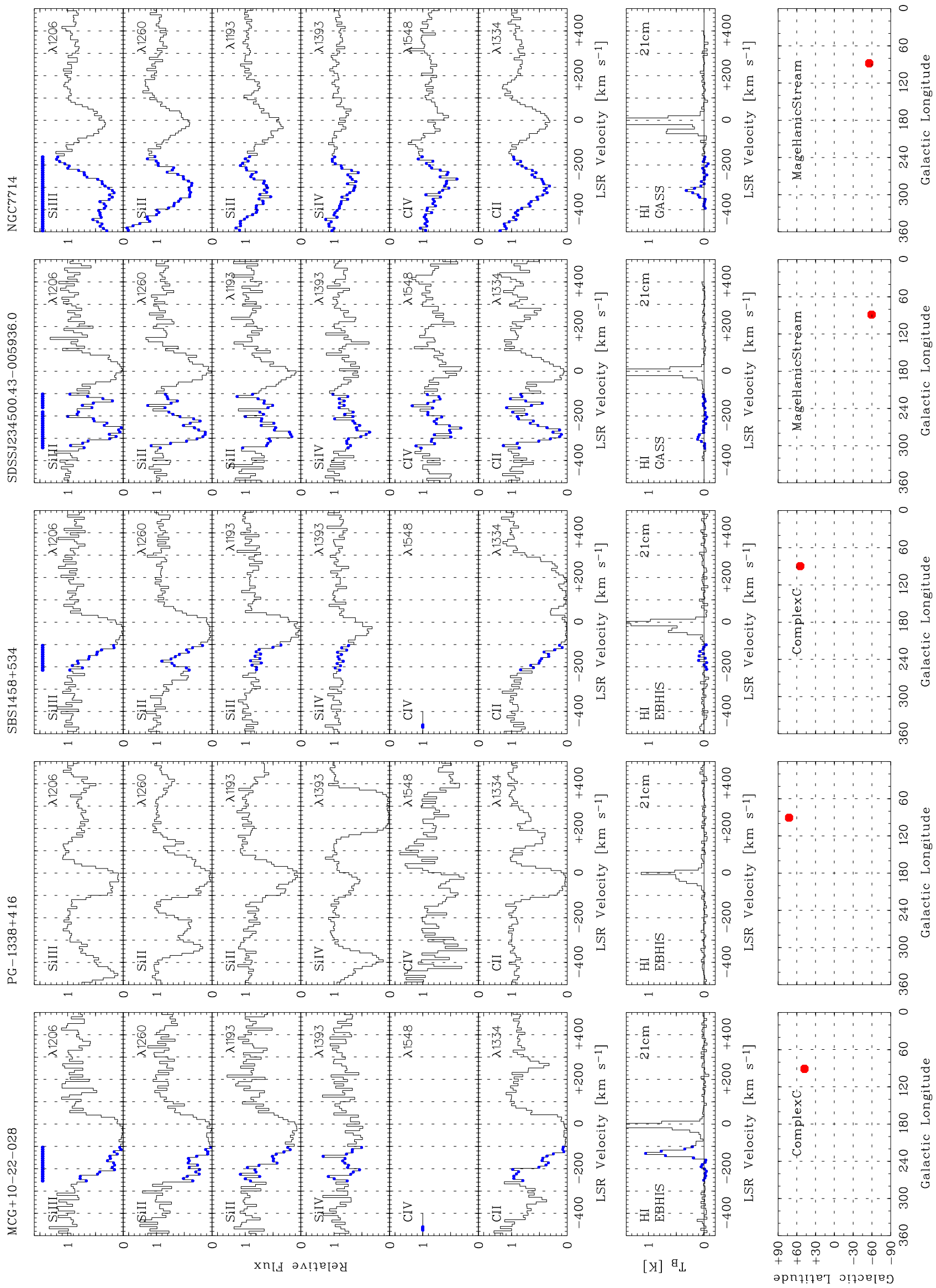

Fig. B.2. continued. 
P. Richter et al.: The Milky Way CGM in absorption
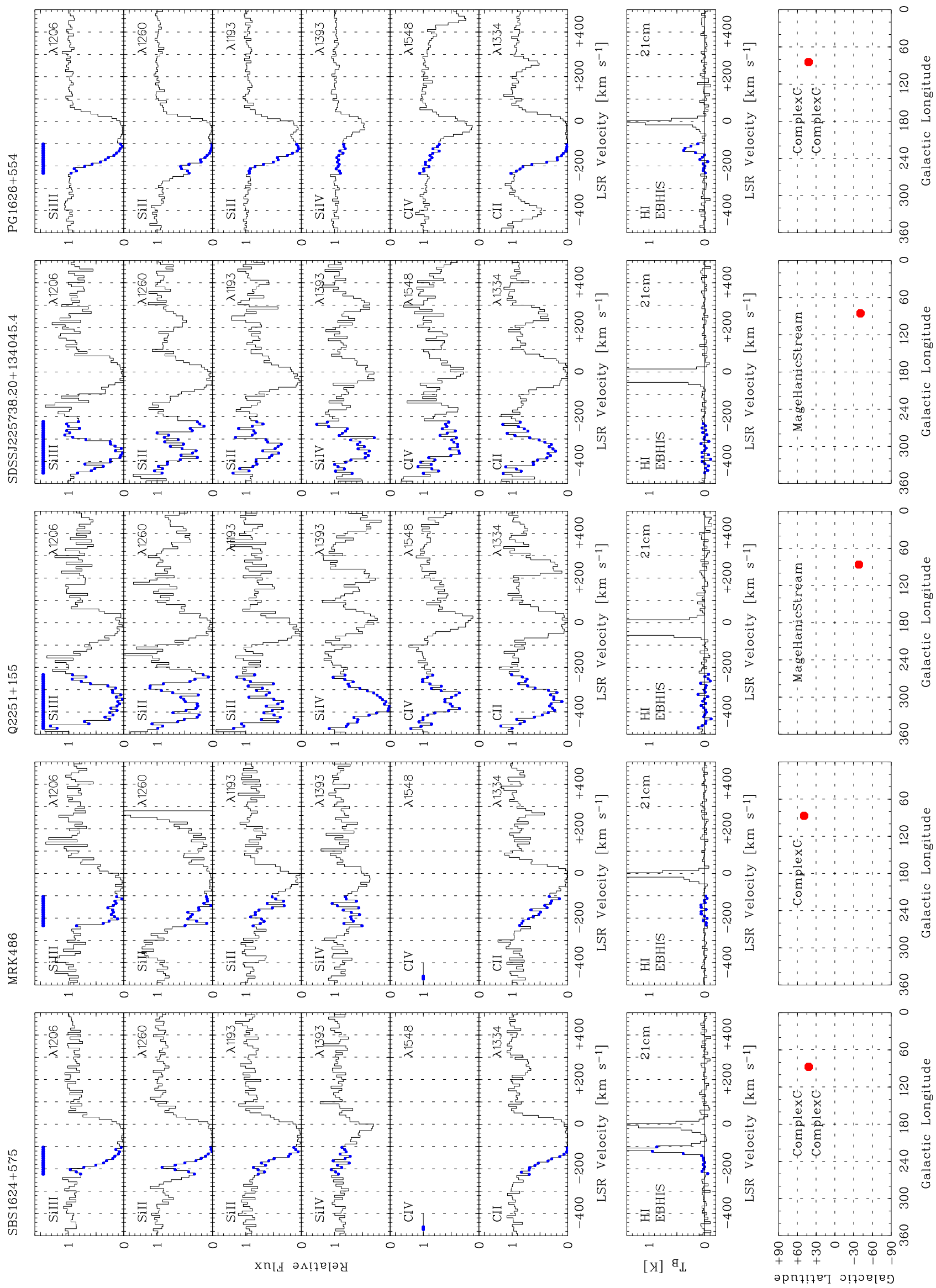

Fig. B.2. continued. 

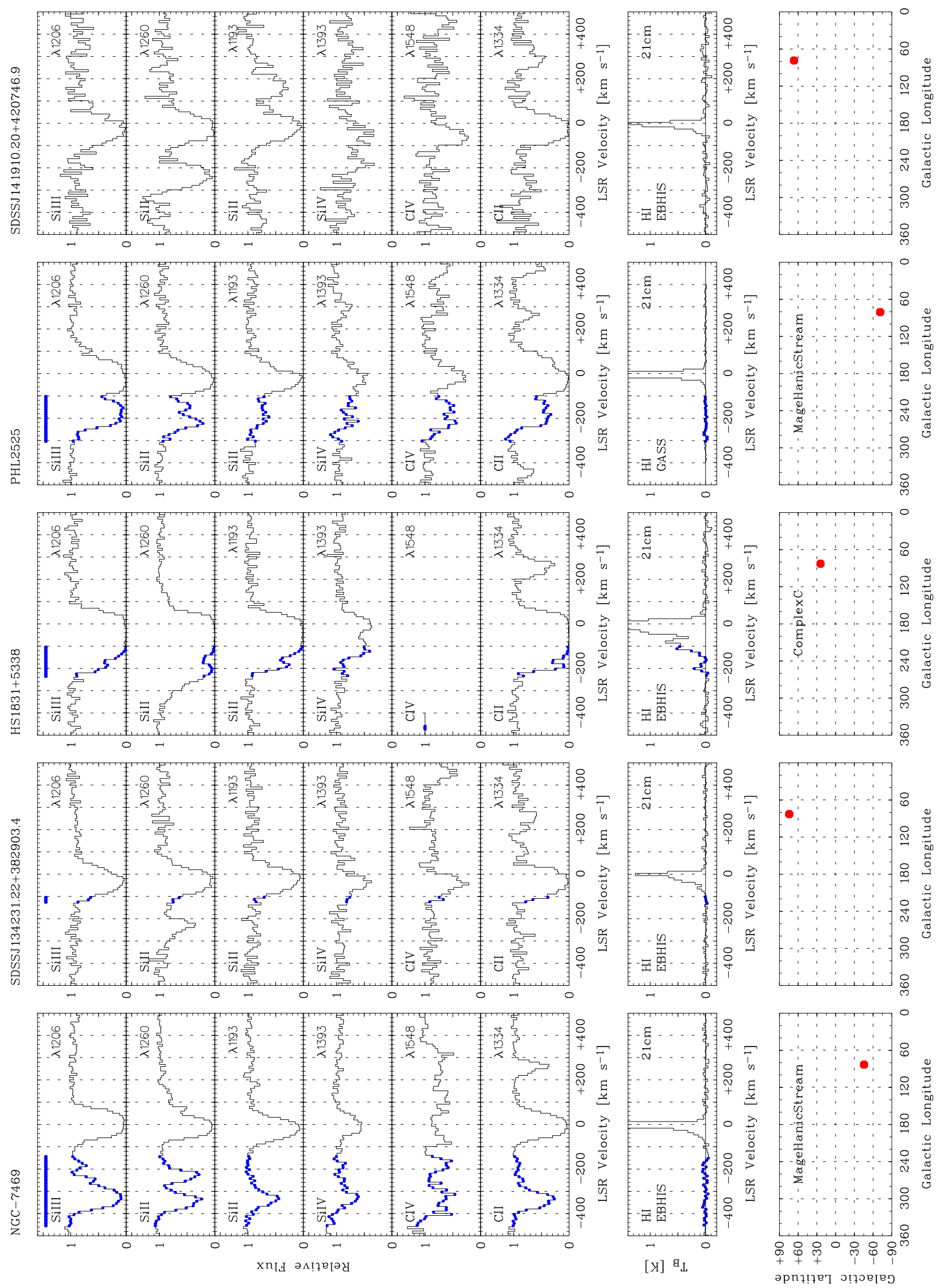

Fig. B.2. continued. 
P. Richter et al.: The Milky Way CGM in absorption
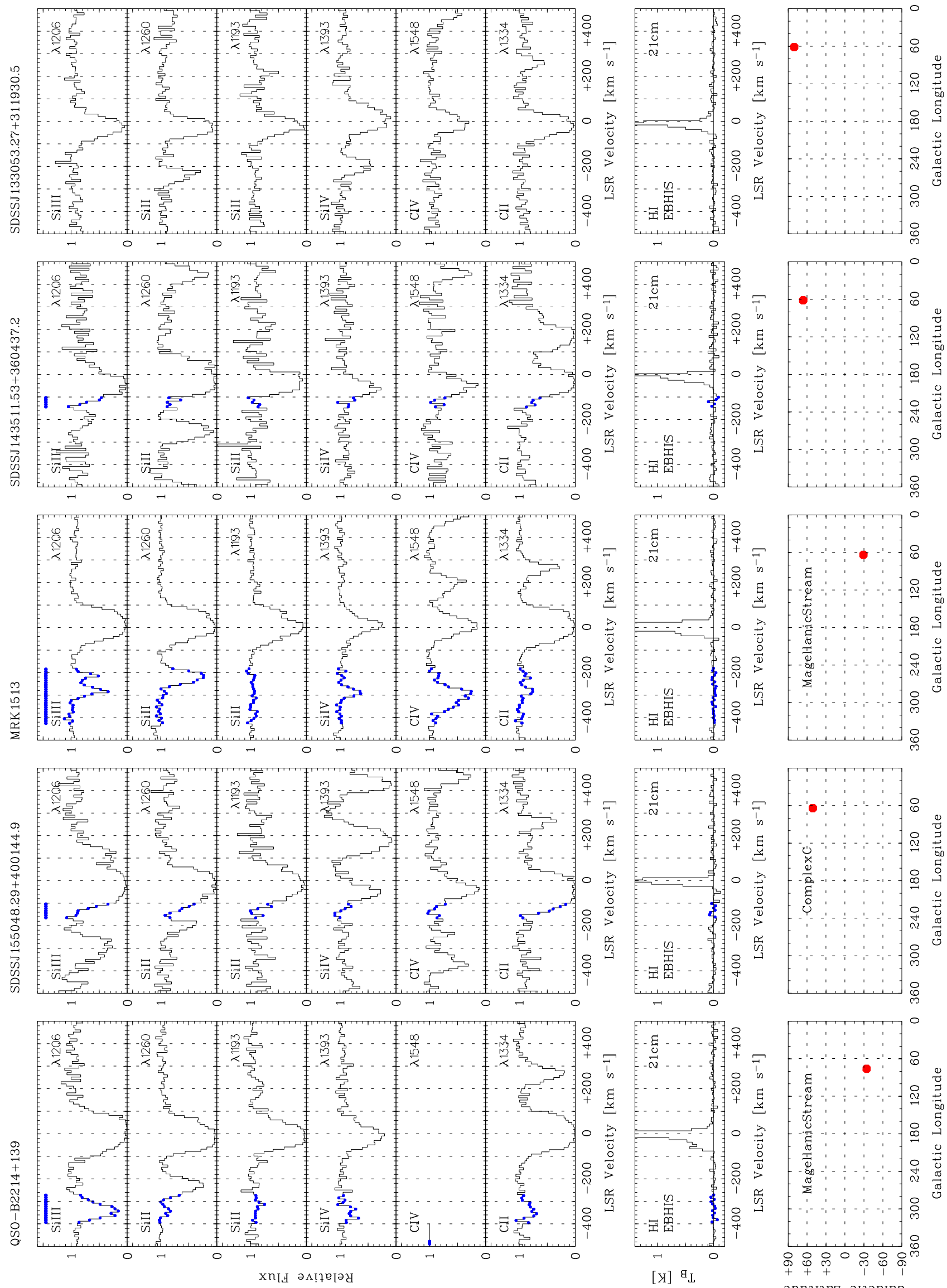

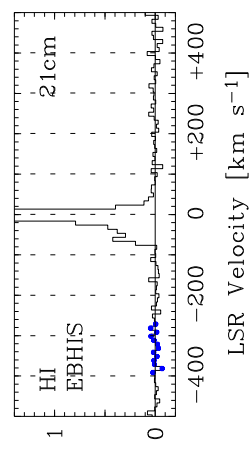

[y] ${ }^{\mathrm{L}} \mathrm{L}$

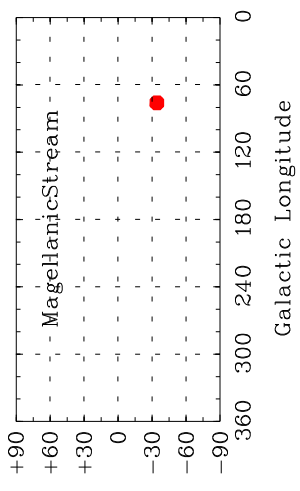

әрпาเาет опұวетер

Fig. B.2. continued. 

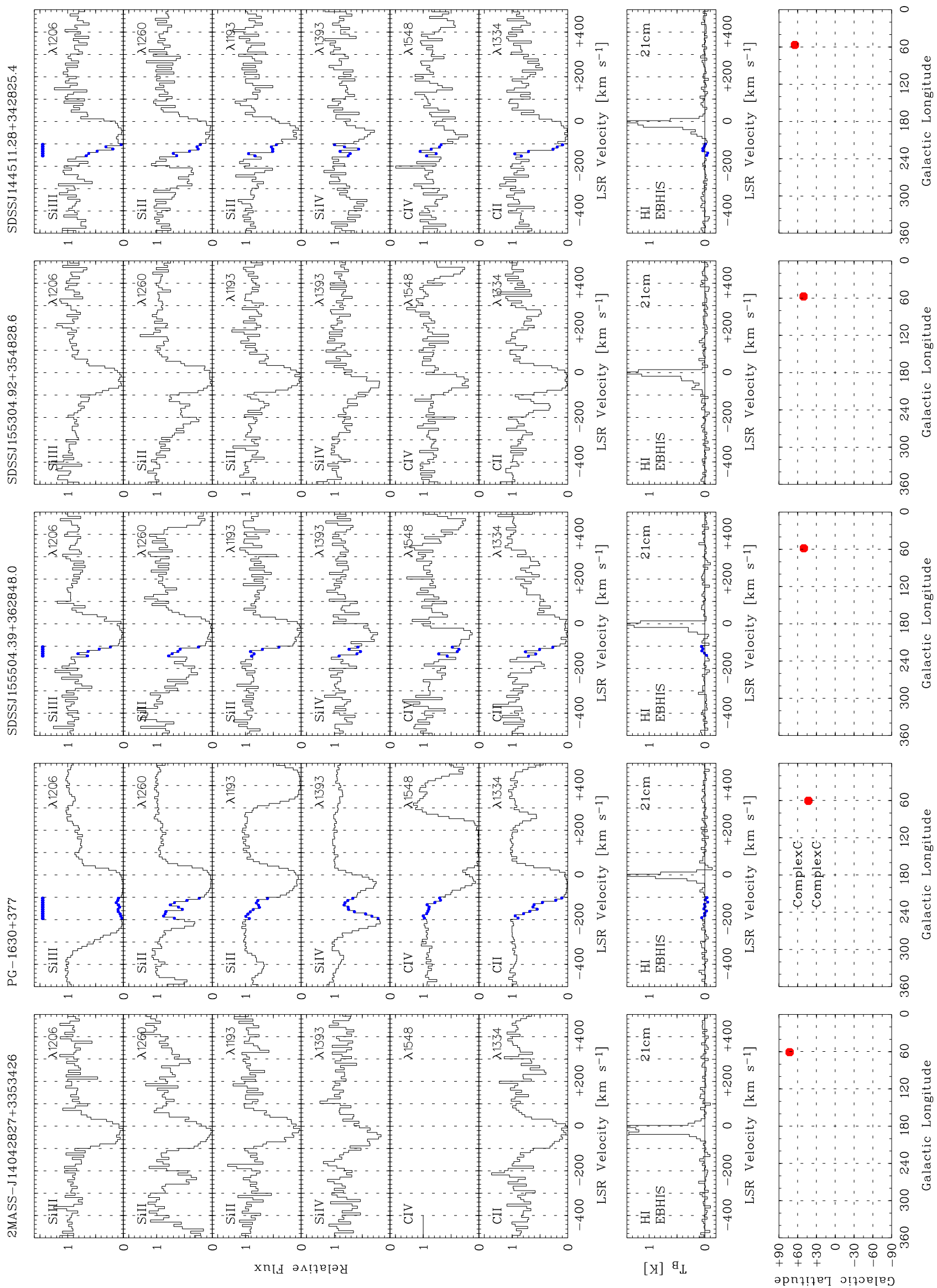

Fig. B.2. continued. 
P. Richter et al.: The Milky Way CGM in absorption
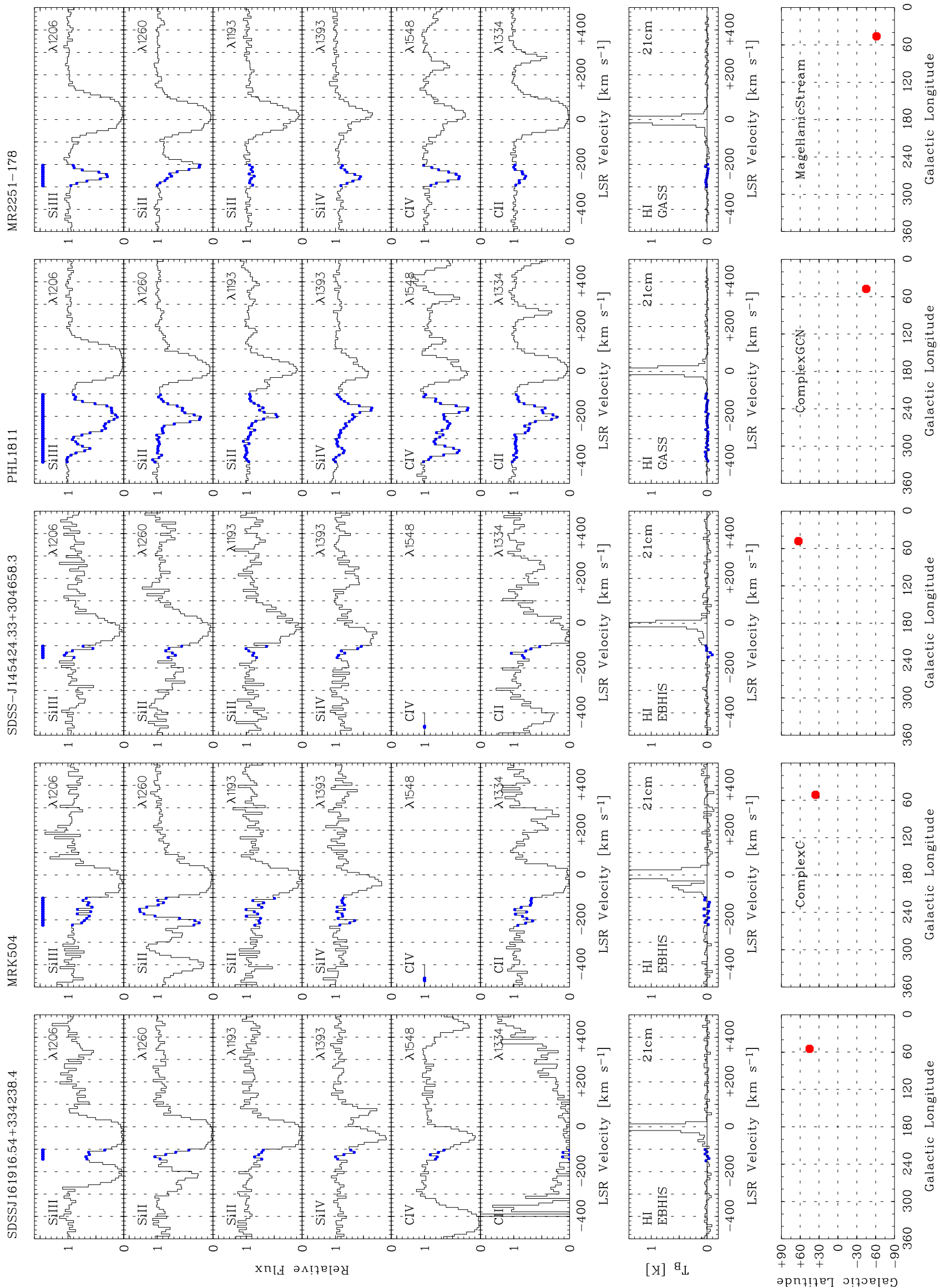

Fig. B.2. continued. 

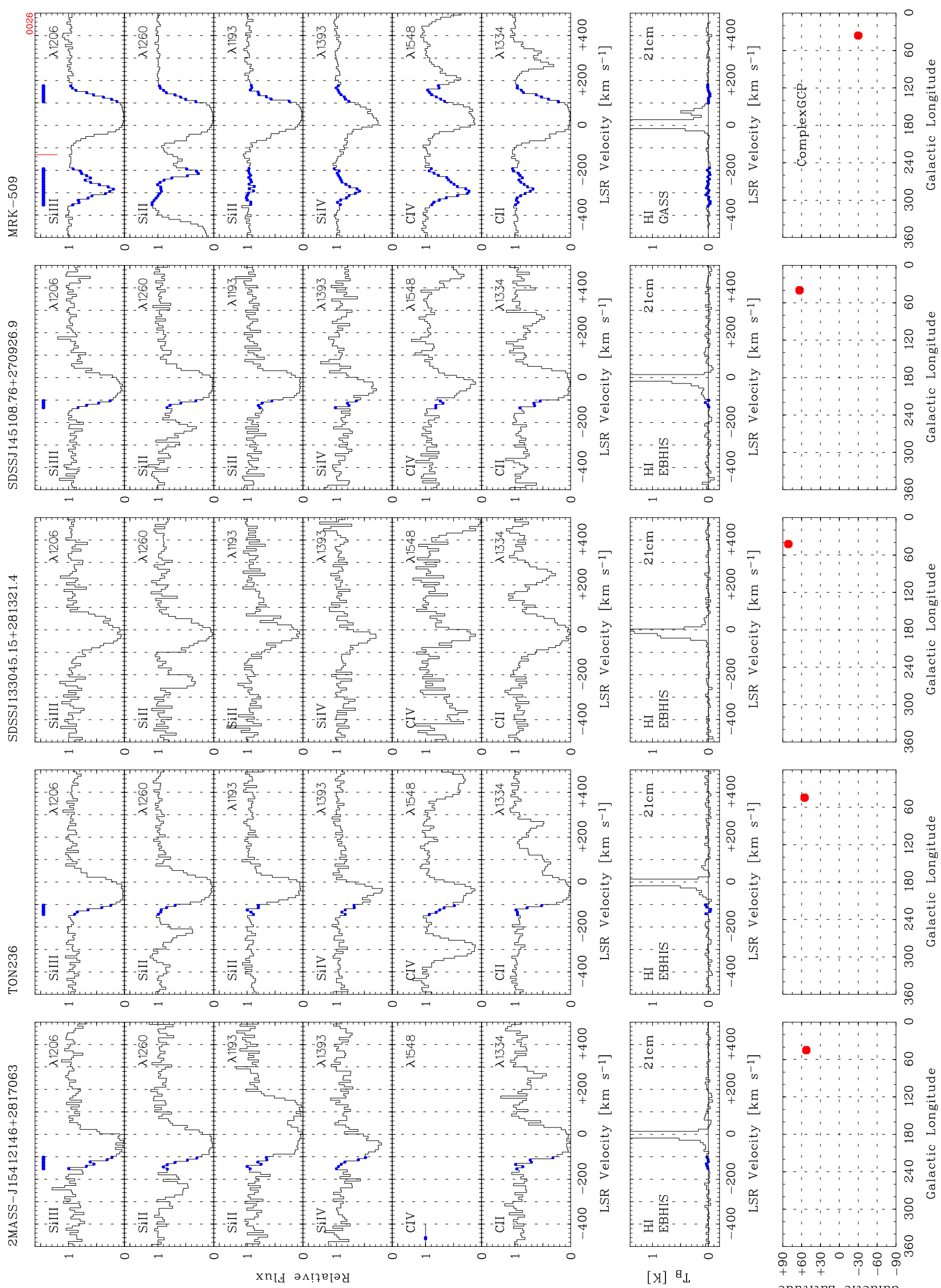

Fig. B.2. continued. 
P. Richter et al.: The Milky Way CGM in absorption
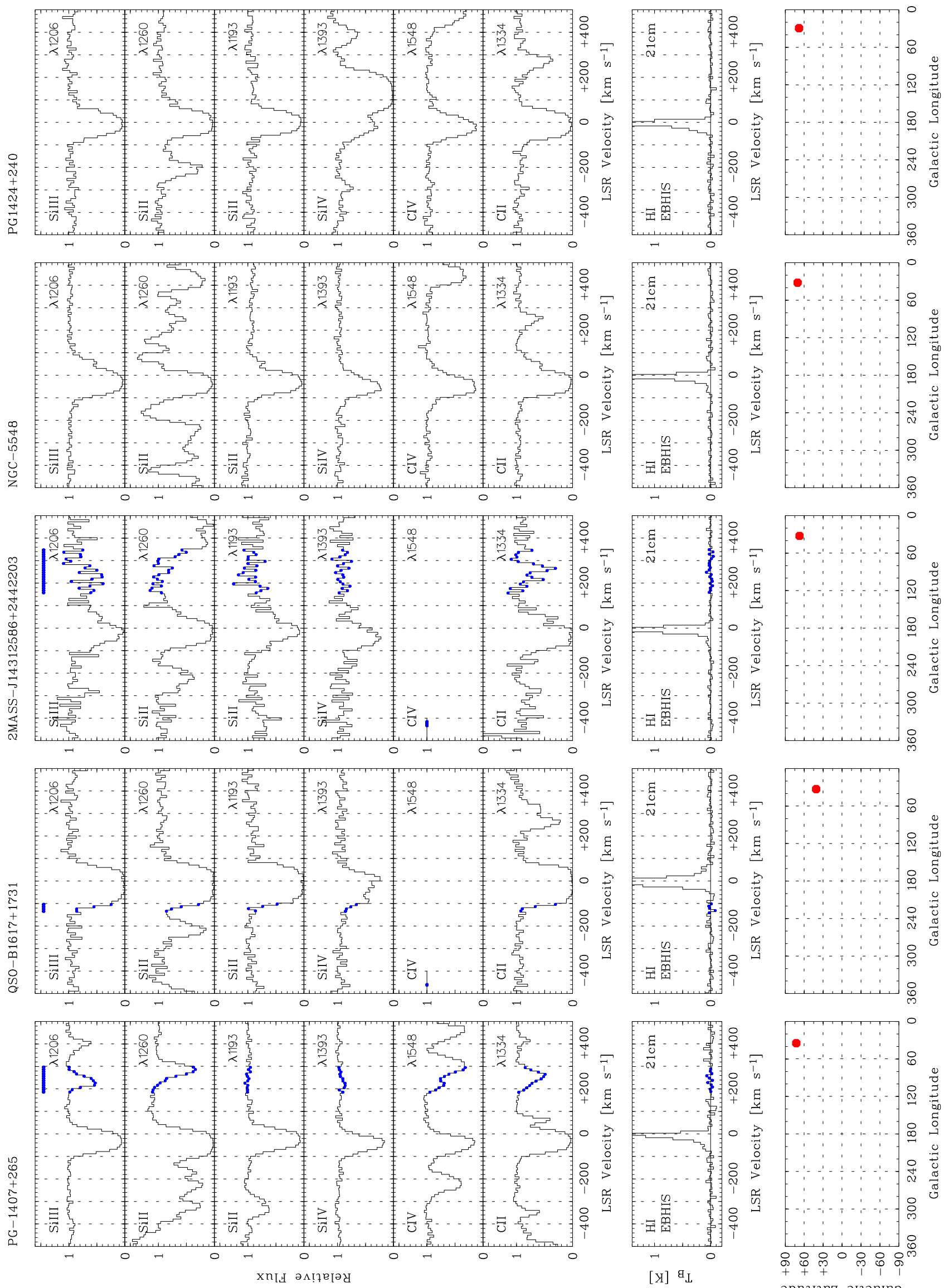

Fig. B.2. continued. 

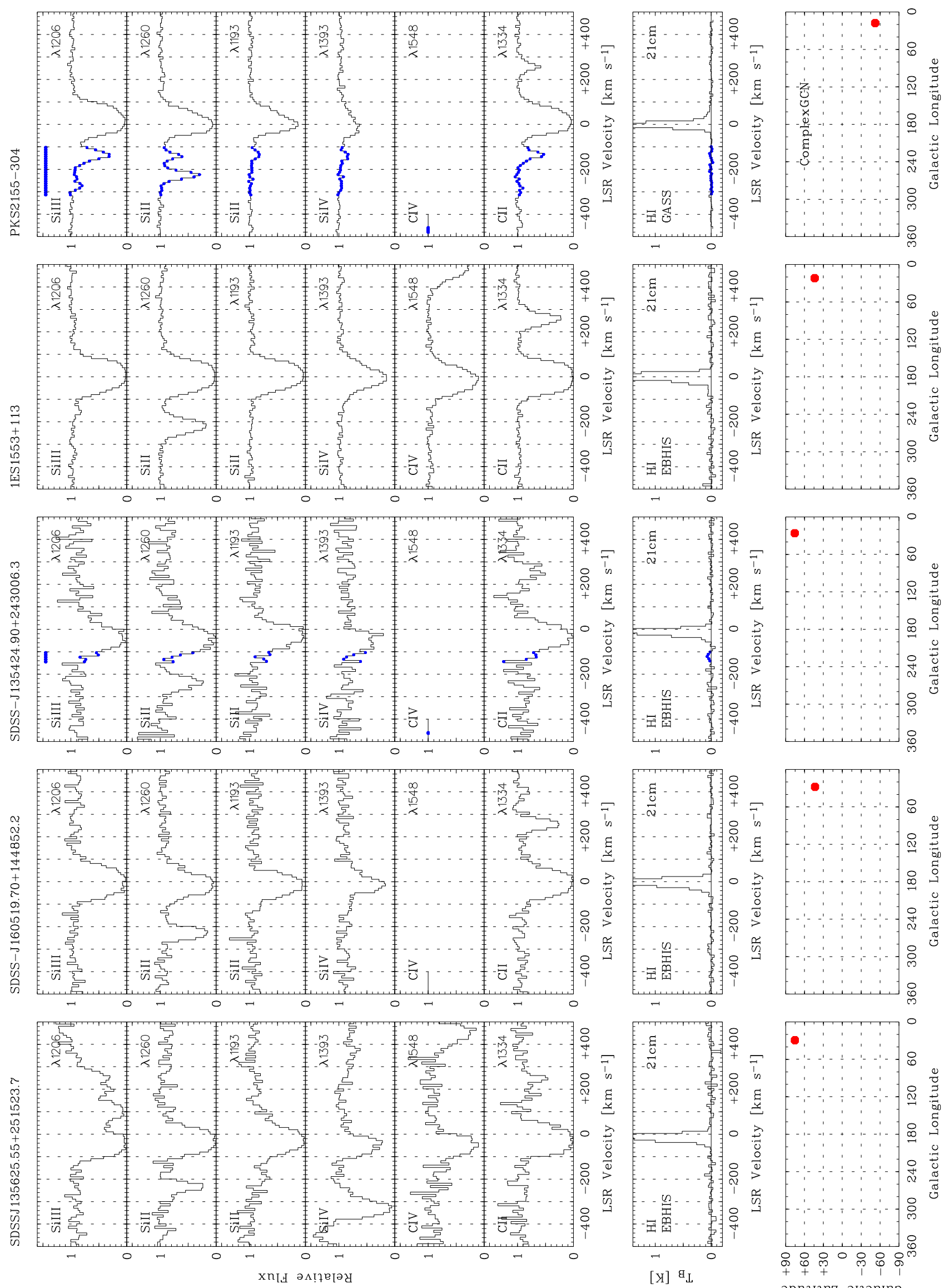

Fig. B.2. continued. 
P. Richter et al.: The Milky Way CGM in absorption
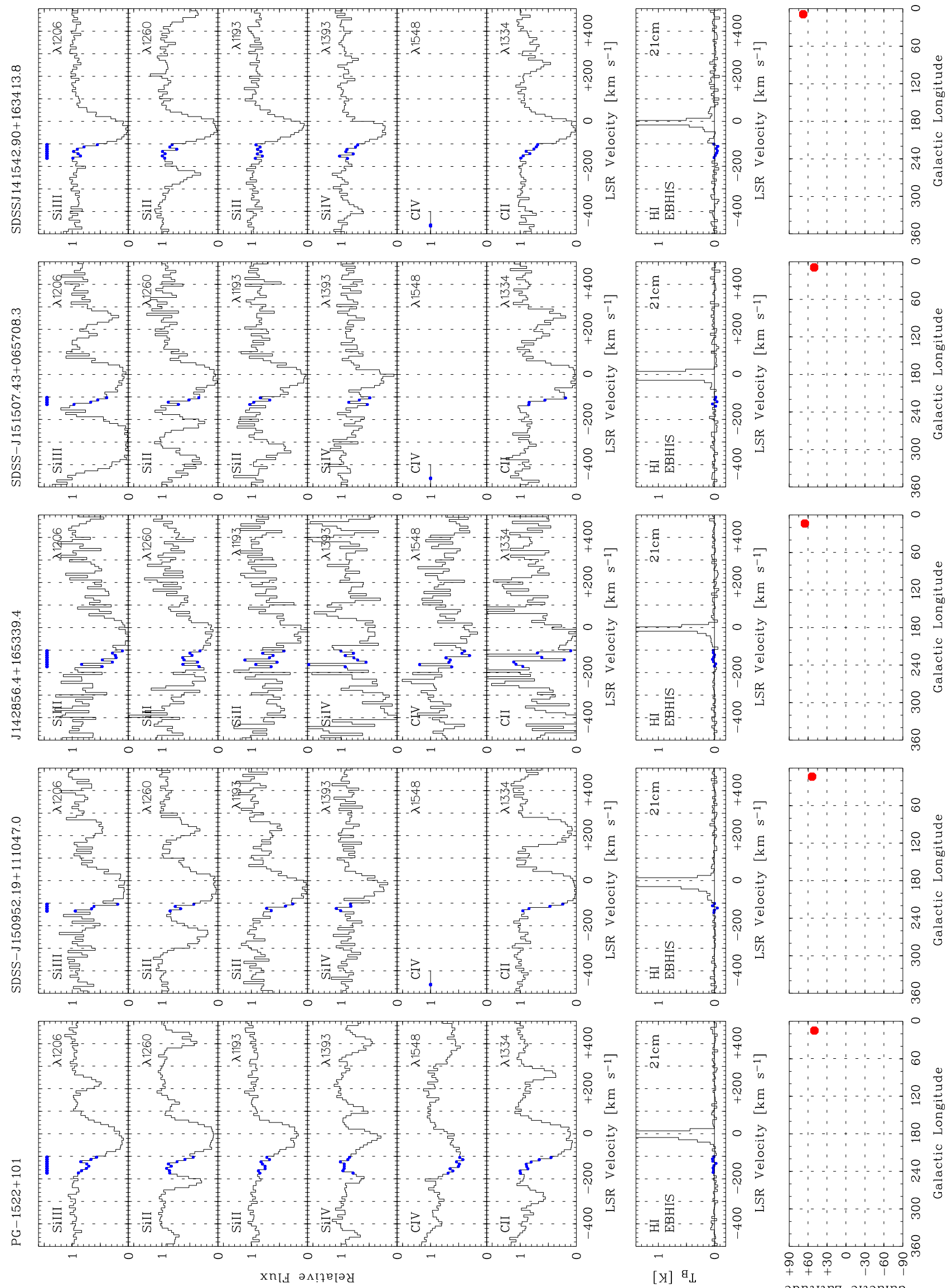

Fig. B.2. continued. 

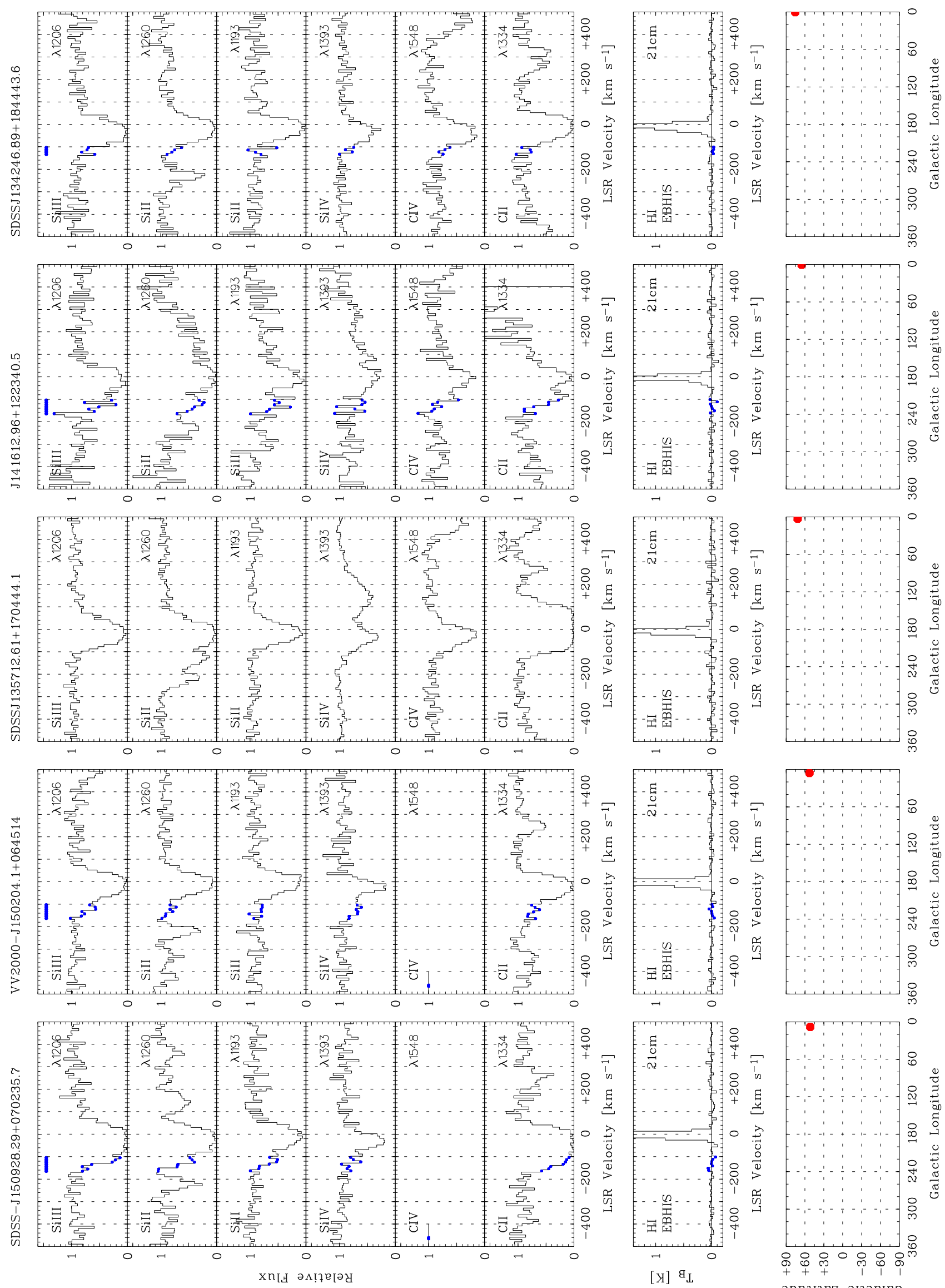

Fig. B.2. continued. 